\title{
U.S. Electric Utility Demand-Side Management 1993
}

\section{July 1995}

\section{Energy Information Administration}

Office of Coal, Nuclear, Electric and Alternate Fuels.

U.S. Department of Energy

Washington, DC 20585



This report was prepared by the Energy Information Administration, the independent statistical and analytical agency within the Department of Energy. The information contained herein should not be construed as advocating or reflecting any policy position of the Department of Energy or any other organization. 


\section{Preface}

The U.S. Electric Utility Demand-Side Management report is prepared by the Coal and Electric Data and Renewables Division; Office of Coal, Nuclear, Electric and Alternate Fuels; Energy Information Administration (EIA); U.S. Department of Energy. The report presents comprehensive information on electric power industry demand-side management (DSM) activities in the United States at the national, regional, and utility levels. The objective of the publication is to provide industry decision makers, government policy makers, analysts, and the general public with historical data that may be used in understanding demand-side management as it relates to the U.S. electric power industry. The first chapter, "Profile: U.S. Electric Utility Demand-Side Management," presents a general discussion of DSM, its history, current issues, and a review of key statistics for the year. Subsequent chapters present discussions and more detailed data on energy savings, peakload reductions and costs attributable to DSM. Included in this year's publication are two feature articles, "The Status of U.S. Electric Utility Demand-Side Management," and "Evaluation and Verification of Demand-Side Management Programs."

\section{Target Audience}

In the private sector, the majority of users are researchers, analysts, and ultimately the policymaking and decisionmaking members of electric utility companies. Financial and investment institutions, economic development organizations interested in new power plant construction, special interest groups, lobbyists, electric power associations, and the news media are all prospective users of the U.S. Electric Utility Demand-Side Management report.

In the public sector, users include analysts, researchers, statisticians, and other professionals engaged in regulatory, policy, and program areas for Federal, State, and local governments. The Congress, other legislative bodies, State utility commissions, and other government groups share an interest in general trends and specific DSM data. This report can be used in analytic studies to evaluate new or existing legislation.

\section{Source of Data}

Data published in the U.S. Electric Utility DemandSide Management report are compiled from the Form EIA-861, "Annual Electric Utility Report." The Form EIA-861 is a census of electric utilities in the United States, its territories, and Puerto Rico. It is used to collect annual data on the production, sales, revenue from sales, and trade of electricity, as well as demandside management data from approximately 3,250 electric utilities in the United States. Schedule V, "Demand-Side Management Information," of the Form EIA-861 collects demand-side management data.

Questions regarding the contents of this document may be directed to:

Coal and Electric Data and Renewables Division

Energy Information Administration, EI-52

U.S. Department of Energy

1000 Independence Avenue, S.W.

Washington, DC 20585

Fax phone number (202) 254-5765

Questions of a general nature may be directed to:

Howard Walton (202/254-5500),

Internet E-Mail: hwalton @ eia.doe.gov

Director of the Coal and Electric Data and Renewables Division

Specific information on demand-side management may be directed to:

Linda M. Bromley (202/254-5653),

Internet E-Mail: lbromley @ eia.doe.gov

or

Channele M. Donald (202/254-8571),

Internet E-Mail: cdonald@ eia.doe.gov 


\section{DISCLAIMER}

This report was prepared as an account of work sponsored by an agency of the United States Government. Neither the United States Government nor any agency thereof, nor any of their employees, make any warranty, express or implied, or assumes any legal liability or responsibility for the accuracy, completeness, or usefulness of any information, apparatus, product, or process disclosed, or represents that its use would not infringe privately owned rights. Reference herein to any specific commercial product, process, or service by trade name, trademark, manufacturer, or otherwise does not necessarily constitute or imply its endorsement, recommendation, or favoring by the United States Government or any agency thereof. The views and opinions of authors expressed herein do not necessarily state or reflect those of the United States Government or any agency thereof. 


\section{DISCLAIMER}

Portions of this document may be illegible in electronic image products. Images are produced from the best available original document. 


\section{Contents}

The Status of U.S. Electric Utility Demand-Side Management vii

Evaluation and Verification of Demand-Side Management Programs xvii

Profile: U.S. Electric Utility Demand-Side Management

Energy Savings

Peakload Reduction

Cost

Appendices

A. Technical Notes

B. Glossary

C. Abbreviations and Acronyms 


\section{Tables}

Page

1. U.S. Electric Utility DSM Program Energy Savings, Actual and Potential Peakload Reductions, and Cost, 1989 Through 1993

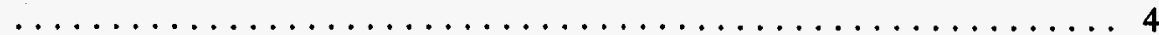

2. U.S. Electric Utility DSM Program Energy Savings by Class of Ownership, 1989 Through 1993, 1994 and 1998

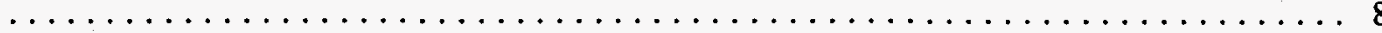

3. U.S. Electric Utility DSM Program Energy Savings by Program Category, 1992, 1993, 1994, and 199810

4. Number of U.S. Electric Utilities with DSM Energy Efficiency Programs by End Uses and Program

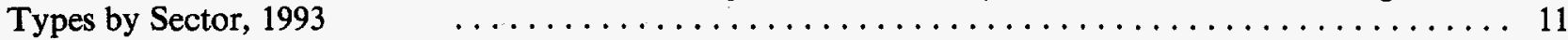

5. U.S. Electric Utility DSM Program Energy Savings by Sector, 1992 and $1993 \quad \ldots \ldots \ldots \ldots \ldots 11$

6. U.S. Electric Utility Incremental Energy Savings by Class of Ownership, 1992 and $1993 \quad \ldots \ldots 12$

7. U.S. Electric Utility Incremental Energy Savings by Program Category, 1992 and $1993 \quad \ldots . .12$

8. U.S. Electric Utility Incremental Energy Savings by Sector, 1992 and $1993 \quad \ldots \ldots \ldots \ldots \ldots 12$

9. U.S. Electric Utility Energy Savings by North American Electric Reliability Council Region and Hawaii by Class of Ownership, 1992, 1993, 1994, and $1998 \quad \ldots \ldots \ldots \ldots \ldots \ldots \ldots \ldots \ldots$

10. U.S. Electric Utility Energy Savings by North American Electric Reliability Council Region and

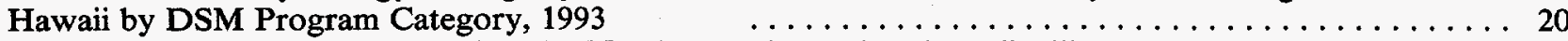

11. U.S. Electric Utility Energy Savings by North American Electric Reliability Council Region and Hawaii by Class of Ownership and Sector, $1993 \quad \ldots \ldots \ldots \ldots \ldots \ldots \ldots \ldots \ldots \ldots \ldots$

12. U.S. Electric Utility Actual and Potential Peakload Reductions by Class of Ownership, 1989 Through

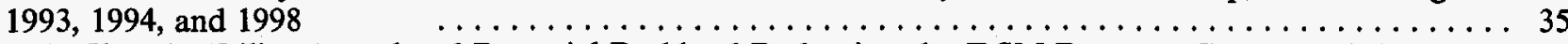

13. U.S. Electric Utility Actual and Potential Peakload Reductions by DSM Program Category, 1992,

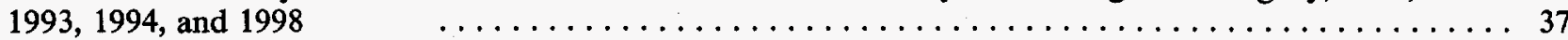

14. U.S. Electric Utility Actual and Potential Peakload Reductions by Sector, 1992 and $1993 \quad \ldots 38$

15. U.S. Electric Utility Incremental Actual Peakload Reductions by Class of Ownership, 1992 and 1993.39

16. U.S. Electric Utility Incremental Actual Peakload Reductions by DSM Program Category, 1992 and

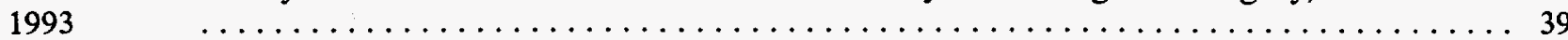

17. U.S. Electric Utility Incremental Actual Peakload Reductions by Sector, 1992 and $1993 \quad \ldots 39$

18. U.S. Electric Utility Actual and Potential Peakload Reductions by North American Electric Reliability Council Region and Hawaii by Utility, 1992, 1993, 1994, and $1998 \quad \ldots \ldots \ldots \ldots \ldots \ldots \ldots .40$

19. U.S. Electric Utility Actual Peakload Reductions by North American Electric Reliability Council Region and Hawaii by DSM Program Category, $1993 \quad \ldots \ldots \ldots \ldots \ldots \ldots \ldots \ldots \ldots . \ldots$

20. U.S. Electric Utility Actual Peakload Reductions by North American Electric Reliability Council Region and Hawaii by Class of Ownership and Sector, $1993 \quad \ldots \ldots \ldots \ldots \ldots \ldots \ldots \ldots . \ldots 5$

21. U.S. Electric Utility DMS Program Costs by Class of Ownership, 1989 Through 1993, 1994, and 199865

22. U.S. Electric Utility DSM Program Costs by North American Electric Reliability Council Region and Hawaii by Class of Ownership, 1992, 1993, 1994, and $1998 \quad \ldots \ldots \ldots \ldots \ldots \ldots \ldots \ldots 6$

23. U.S. Electric Utility DSM Program Direct Utility Costs by North American Electric Reliability Council Region and Hawaii by DSM Program Category, $1993 \quad \ldots \ldots \ldots \ldots \ldots \ldots \ldots \ldots 74$

24. U.S. Electric Utility DSM Program Indirect Utility Costs by North American Electric Reliability Council Region and Hawaii by Cost Category, $1993 \quad \ldots \ldots \ldots \ldots \ldots \ldots \ldots \ldots \ldots \ldots$

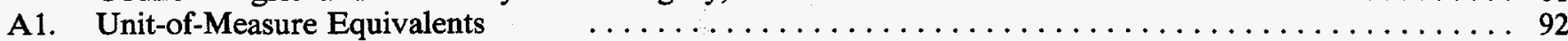

A2. Corrections to DSM Data, 1989 through $1992 \quad \ldots \ldots \ldots \ldots \ldots \ldots \ldots \ldots \ldots \ldots \ldots \ldots . \ldots \ldots$ 


\section{Illustrations}

Page

1. Number of U.S. Electric Utilities With and Without DSM Programs, 1993

2. U.S. Electric Utility DSM Program Incremental and Annual Effects for Energy Savings and Actual and Potential Peakload Reductions, 1993



3. Energy Savings as a Percentage of Retail Sales by U.S. Electric Utilities with DSM Energy Savings Programs and by Class of Ownership, 1993 The Top 25, 50, and 100 U.S. Electric Utilities with the Greatest DSM Program Energy Savings by Class of Ownership, 1993

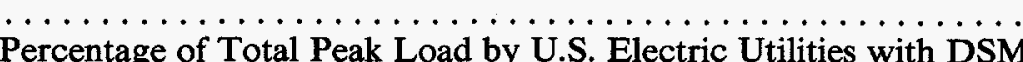

5. Actual Peakload Reductions as a Percentage of Total Peak Load by U.S. Electric Utilities with DSM Peakload Reduction Programs and by Class of Ownership, 1993 10

6. The Top 25, 50, and 100 U.S. Electric Utilities with the Greatest DSM Program Peakload Reductions by Class of Ownership, 1993

7. U.S. Electric Utility Actual and Potential Peakload Reductions by DSM Program Category, 1993

8. U.S. Electric Utility DSM Program Costs as a Percentage of Retail Revenue by Number of Utilities with DSM Costs, 1993

9. The Top 25, 50, and 100 U.S. Electric Utilities with the Greatest DSM Program Costs by Class of Ownership, 1993 



\section{The Status of U.S. Electric Utility Demand-Side Management}

\section{Background}

Demand-side management (DSM) programs sponsored by electric utilities have been increasingly offered to utility customers in recent years. DSM includes utility efforts to reduce the amount and affect the timing of customer electricity use. Programs that reduce the amount of electricity use (measured in kilowatthours) focus on improving the technical and operational efficiency of customers' use of electricity. Programs that affect the timing of electricity use (measured in kilowatts) focus on load management programs, such as when the utility directly or indirectly (through contracts) controls equipment at the customer site and electricity-pricing options that vary the price of electricity with time of use.

The Energy Information Administration (EIA) collects DSM data in an annual census of electric utilities, Form EIA-861, "Annual Electric Utility Report." The data collected by EIA for the 1993 reporting year represent the most complete and current information available on the status of DSM in the United States. The DSM energy savings, peakload reduction, and expenditures from 1992 to 1993 and projections for 1994 and 1998 are discussed in this article. ${ }^{1}$ This discussion proceeds from the aggregate (U.S. totals) to the disaggregate (NERC region, State; and utility level).

Increasing competition in the electric power industry may affect the type and level of DSM programs and expenditures in the future. Utilities concerned about the adverse effects of DSM programs on electricity prices are reducing the scope of their programs. DSM trade media reports on DSM budget reductions at some utilities are stimulating discussion on the role of DSM in a more competitive environment.

\section{DSM at the National Level}

Utility expenditures on DSM programs increased 17 percent from $\$ 2.3$ billion in 1992 to $\$ 2.7$ billion in 1993 (Table FE1). Projected expenditures for 1994 were an additional 9 percent increase. ${ }^{2}$ Energy savings also increased to 45,294 million $\mathrm{kWh}$ in 1993, 27 percent greater than the 1992 level of 35,563 million $\mathrm{kWh}$; potential peakload reductions increased to 39,508 MW, 22 percent higher than the 1992 level of $32,442 \mathrm{MW}^{3}$

In 1993, utilities spent 1.4 percent of their retail revenues for DSM programs. Energy savings resulting from these DSM programs reduced sales of electricity by 1.6 percent, and potential reduction of peak load was 6.8 percent (Figure FE1). The projections for 1994 and 1998 also predict increased spending, energy savings, and potential peakload reduction, although the rates of increase are declining for expenditures and peakload reductions, but not for energy savings (Table FE1).

EIA collects DSM data using six program categories: energy efficiency, direct load control, interruptible load control, other load management, ${ }^{4}$ other DSM programs, and load building. ${ }^{5}$ Energy-efficiency programs dominated DSM expenditures for all 3 years (Figure FE2), at 66 percent in 1992, 70 percent in 1993, and projected to be 71 percent in 1994. Direct load control and interruptible load programs, which focus on peakload reduction rather than energy savings, were second and third, respectively, in utility DSM expenditures. The relative emphasis on energy savings versus peakload reduction has shifted slightly toward energy saving from 1992 to 1994.

\footnotetext{
1 Eric Hirst's support and contribution to the Electric Operating and Financial Data Branch in preparing this article are greatly appreciated.

2 Normalized for inflation, "real" expenditures on DSM increased by 14 percent from 1992 to 1993 and are projected to increase by 6 percent from 1993 to 1994.

3 The energy savings and peakload reduction data presented in this article are annual. Annual numbers are the total effects in energy use and peak load for a particular year caused by all participants in the DSM programs that are in effect during a given year.

4 Other load management refers to programs other than direct load control and interruptible load that limit or shift peak load from on-peak to off-peak time periods. It includes technologies that primarily shift all or part of a load from one time of day to another and secondarily may have an impact on energy consumption. Examples include space heating and water heating storage systems, cool storage systems, and load limiting devices in energy management systems. This category also includes programs that aggressively promote time-of-use rates and other innovative rates such as real-time pricing. These rates are intended to reduce consumer bills and shift hours of operation of equipment from on-peak to off-peak periods through the application of time-differentiated rates.

5 Although DSM includes programs that increase energy use (i.e., load building) as well as those that reduce consumption, only the latter are discussed in this article.
} 
Table FE1. U.S. Electric Utility DSM Program Annual Effects and Expenditures and Percentage of Retail Electric Revenue and Sales and Summer Peak Load, 1989 Through 1993, 1994 and 1998

\begin{tabular}{c|c|c|c|c|c|c}
\hline & \multicolumn{2}{|c|}{ Program Expenditures } & \multicolumn{2}{c|}{ Energy Savings } & \multicolumn{2}{c}{$\begin{array}{c}\text { Potential Peakload } \\
\text { Reduction }\end{array}$} \\
\hline Year $^{1}$ & Million \$ & Percent $^{2}$ & GWh/year & Percent $^{2}$ & MW & Percent $^{2}$ \\
\hline 1989 & 873 & 0.5 & 14,672 & 0.5 & NA & NA \\
1990 & 1,178 & 0.7 & 20,458 & 0.7 & NA & NA \\
1991 & 1,804 & 0.9 & 24,848 & 0.9 & NA & NA \\
1992 & 2,348 & 1.3 & 35,563 & 1.3 & 32,442 & 5.9 \\
1993 & 2,744 & 1.4 & 45,294 & 1.6 & 39,508 & 6.8 \\
1994 & 3,003 & 1.5 & 52,655 & 1.9 & 42,220 & 7.3 \\
1998 & 3,562 & 1.6 & 88,978 & 3.0 & 55,163 & 8.9 \\
\hline
\end{tabular}

The data for 1989 through 1993 are historical results, those for 1994 and 1998 are projections made in the 1993 reporting year.

These percentages reflect, respectively, total U.S. percentage of retail electric revenue spent, retail electricity sales, and summer peak load for each year.

NA $=$ Not available.

Source: Form EIA-861, "Annual Electric Utility Report."

Figure FE1. U.S. Electric Utility DSM Program Expenditures and Effects as a Percentage of Retail Electric Sales and Revenue and Summer Peak Load, 1989 Through 1993, 1994 and 1998

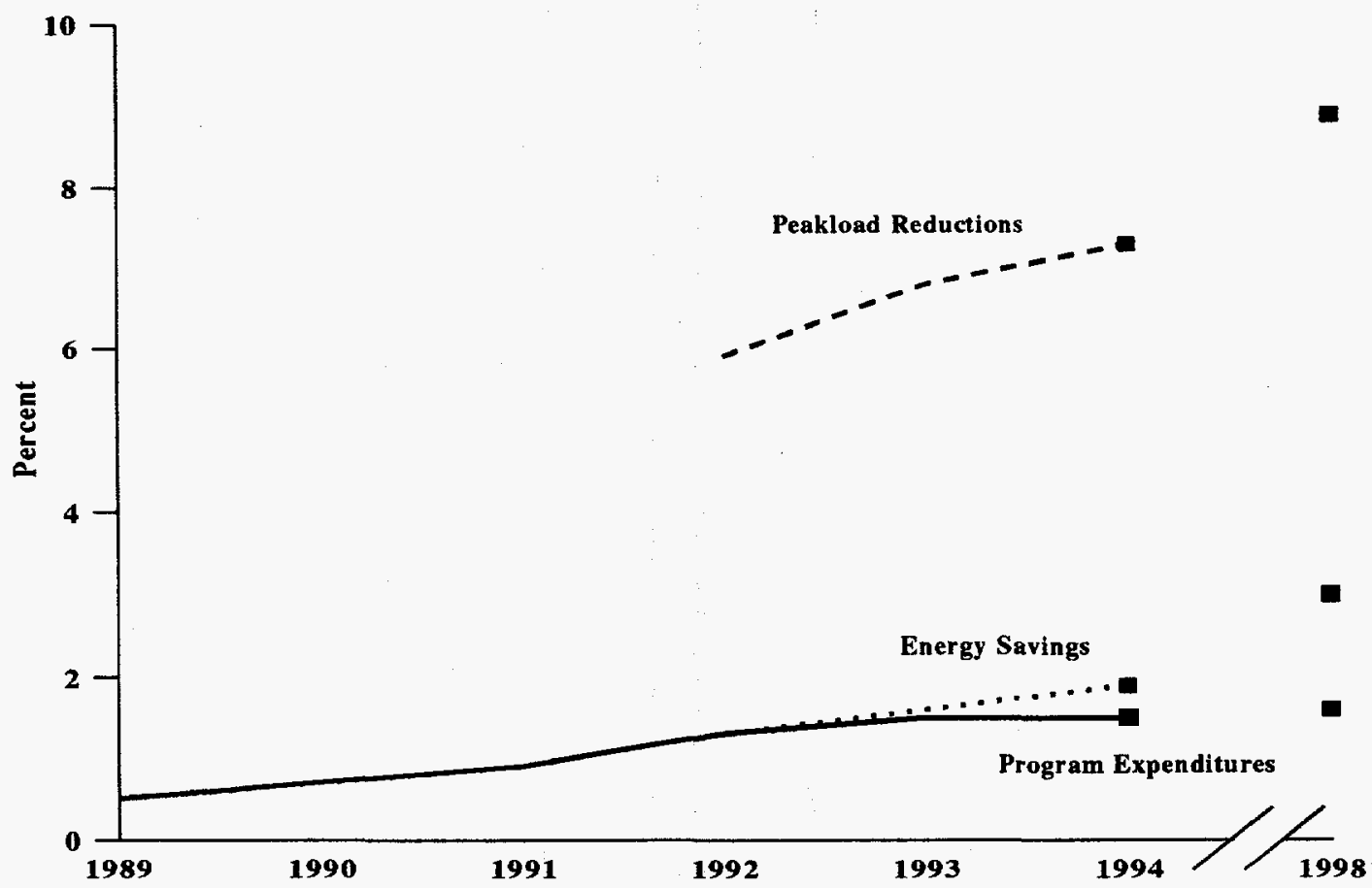

Note: Peakload reduction data not available for 1989-1991. Data for 1994 and 1998 are projected.

Source: Energy Information Administration, Form EIA-861, "Annual Electric Utility Report." 
Figure FE2. U.S. Electric Utility DSM Expenditures by Program Type, 1992, 1993, and 1994

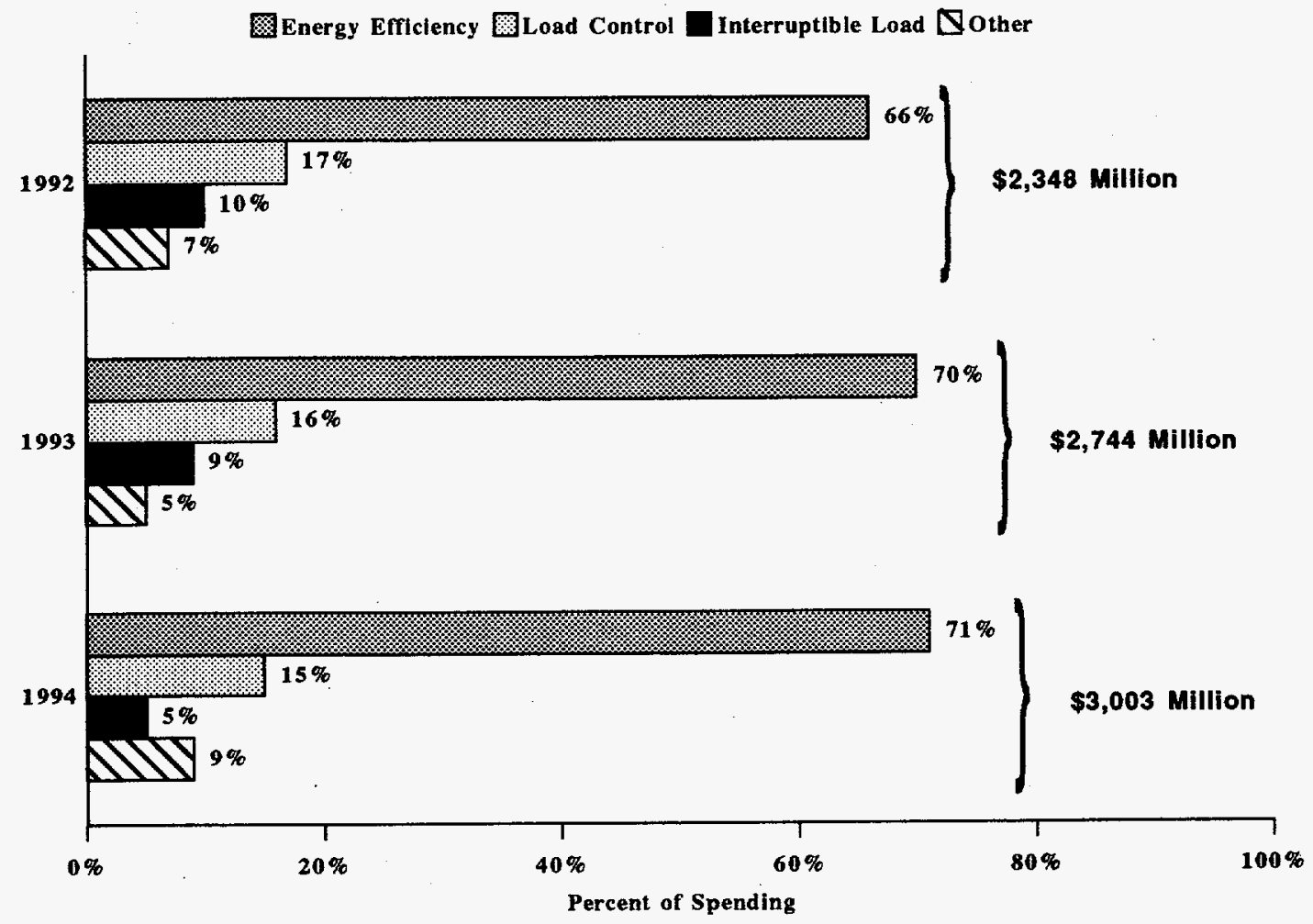

Note: Data for 1994 are projected.

Source: Energy Information Administration, Form EIA-861, "Annual Electric Utility Report."

\section{DSM at the Regional Level}

The level of DSM activity varies among the North American Electric Reliability Council (NERC) regions. DSM expenditures increased in eight of the regions from 1992 to 1993 and electric utilities projected increased expenditures to continue in seven regions from 1993 to 1994 in both absolute terms and relative to retail revenues (Table FE2 and Figure FE3). In the Mid-America Interconnected Network (MAIN) expenditures decreased from 1992 to 1993 and are projected to decrease relative to revenues in the MAIN, Southwest Power Pool (SPP), and Northeast Power Coordinating Council (NPCC) regions from 1993 to 1994 (Figure FE3). The NPCC and Western Systems Coordinating Council (WSCC) regions accounted for the highest percentage of revenues spent on DSM in 1992 and in 1993.

Energy savings and potential peakload reductions increased in all regions from 1992 to 1993 and in all but one (potential peakload reductions in the Electric Reliability Council of Texas (ERCOT)) were predicted to increase from 1993 to 1994 (Table FE2 and
Figures FE4 and FE5). The NPCC and WSCC regions dominated energy savings as a percent of total retail sales for 1992 and 1993, with both regions predicting growth in energy savings of more than 3.5 percent in 1994. At the other end of the spectrum, the Southwest Power Pool (SPP) and East Central Area Reliability Coordination Agreement (ECAR) regions had energy savings of less than 0.5 percent of sales in 1993. Compared with their 1992 levels of energy savings, the ECAR, ERCOT, MAIN, and MidContinent Area Power Pool (MAPP) regions showed the greatest growth in energy savings (Table FE2).

The MAPP and Southeastern Electric Reliability Council (SERC) regions dominated potential peakload reductions as a percentage of peak load for all three years, with both regions showing peakload reductions of more than 10 percent. Potential peakload reductions were smallest in the ECAR and MAIN regions, with both reporting potential peakload reductions of approximately 5 percent (Figure FE5). Relative to their 1992 levels of peakload reductions, the ECAR and SPP regions showed the greatest expected growth through 1994 (Table FE2). 
Figure FE3. U.S. Electric Utility DSM Program Expenditures as a Percentage of Retail Revenue by NERC Region, 1992, 1993 and 1994

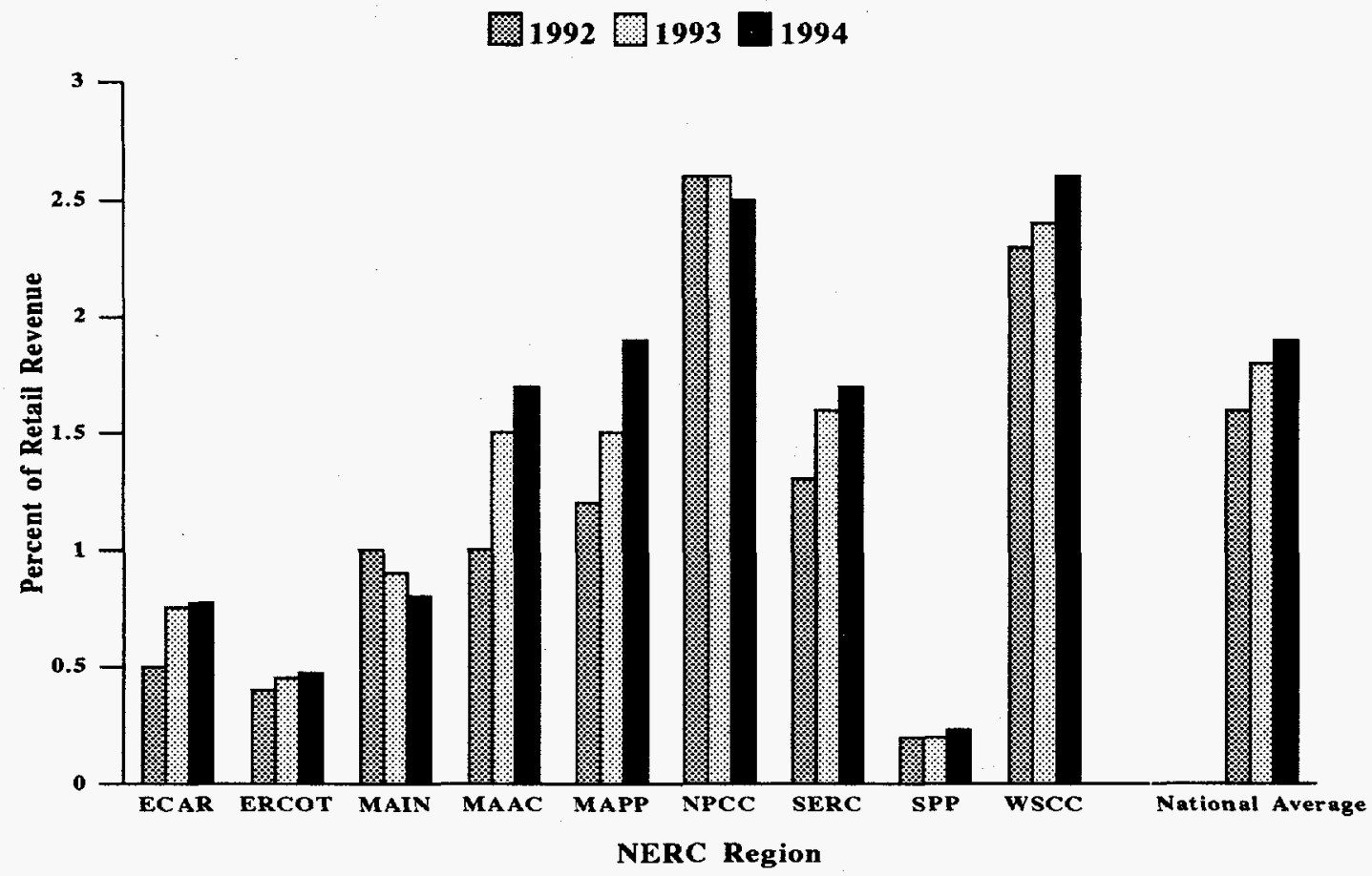

Note: Data for 1994 are projected.

Source: Energy Information Administration, Form EIA-861, "Annual Electrlc Utility Report."

Figure FE4. U.S. Electric Utility Energy Savings as a Percentage of Retall Sales by NERC Region, 1992, 1993, and 1994

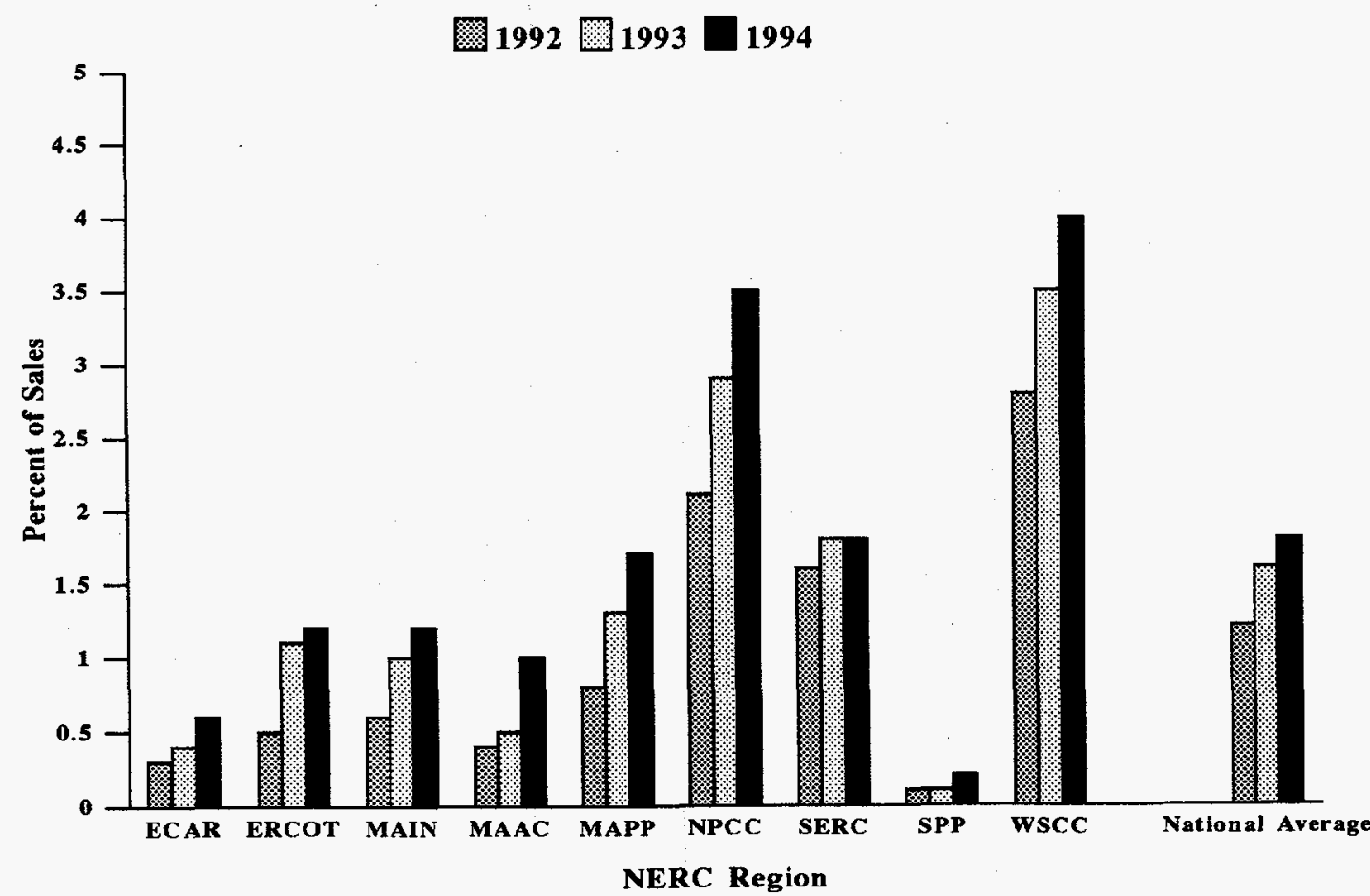

Note: Data for 1994 are projected.

Source: Energy Information Administration, Form EIA-861, "Annual Electric Utility Report." 
Figure FE5. U.S. Electric Utility Potential Peakload Reduction as a Percentage of Summer Peak Load by NERC Region, 1992, 1993, and 1994

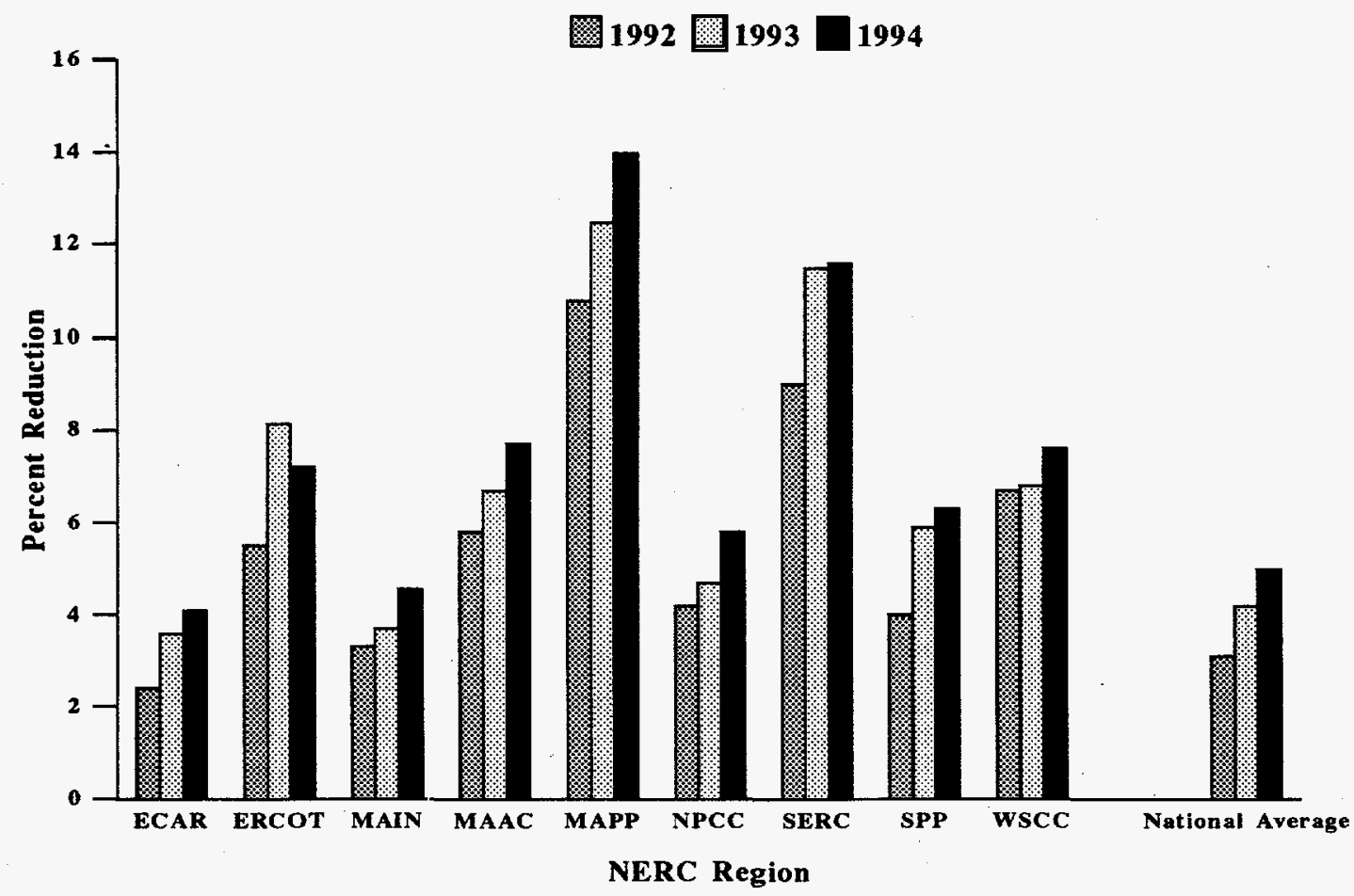

Note: Data for 1994 are projected.

Source: Energy Information Administration, Form EIA-861, "Annual Electric Utility Report."

Table FE2. U.S. Electric Utility Changes in DSM Expenditures and Effects by NERC Region, 1992 to 1993 and 1993 to 1994

\begin{tabular}{l|c|c|c|c|c|c}
\hline & \multicolumn{2}{|c|}{$\begin{array}{c}\text { Percent Change in } \\
\text { DSM Expenditures }\end{array}$} & \multicolumn{2}{c|}{$\begin{array}{c}\text { Percent Change in } \\
\text { Energy Savings }\end{array}$} & \multicolumn{2}{c}{$\begin{array}{c}\text { Percent Change in Potential } \\
\text { Peakload Reduction }\end{array}$} \\
\hline \multicolumn{1}{c}{ Region } & $\mathbf{1 9 9 2 - 1 9 9 3}$ & $\mathbf{1 9 9 3 - 1 9 9 4}$ & $\mathbf{1 9 9 2 - 1 9 9 3}$ & $\mathbf{1 9 9 3 - 1 9 9 4}$ & $\mathbf{1 9 9 2 - 1 9 9 3}$ & $\mathbf{1 9 9 3 - 1 9 9 4}$ \\
\hline ECAR & 43 & 5 & 58 & 40 & 25 & 14 \\
ERCOT & 12 & 5 & 126 & 9 & 48 & -12 \\
MAIN & -4 & -2 & 75 & 20 & 13 & 23 \\
MAAC & 47 & 15 & 21 & 91 & 16 & 17 \\
MAPP & 21 & 25 & 68 & 31 & 17 & 13 \\
NPCC & 4 & 0 & 34 & 23 & 13 & 23 \\
SERC & 26 & 6 & 7 & 4 & 29 & 1 \\
SPP & 8 & -5 & 34 & 25 & 48 & 7 \\
WSCC & 11 & 20 & 24 & 13 & 0 & 12 \\
National Average & 17 & 9 & 27 & 16 & 22 & 7 \\
\hline
\end{tabular}

Note: Data for 1994 are projected.

Source: Energy Information Administration, Form EIA-861, "Annual Electric Utility Report." 


\section{DSM at the State Level}

From 1992 to 1993, DSM expenditures increased in absolute terms by more than 10 percent in 27 States and the District of Columbia, and decreased by 10 percent or more in six States (Figure FE6), including three that spent more than 1 percent of retail revenue on DSM in 1992: California, Maine, and Nevada. DSM expenditures remained approximately constant (i.e., $\mp 10$ percent) in the remaining 17 States.

In 1992, utilities in 11 States and the District of Columbia spent more than 2 percent of retail revenues on DSM. In 1993, utility expenditures on DSM as a share of retail revenue declined in four of these States: California, Connecticut, New York, and Wisconsin. DSM expenditures increased in the other seven States: Florida, Massachusetts, Montana, Oregon, Rhode Island, Vermont, Washington, and in the District of Columbia. For 1994, DSM expenditures as a share of retail revenues are predicted to increase again in these States and the District of Columbia, and also in California.
For 1994, utilities in five States predicted reductions in their DSM expenditures of 10 percent or more. However, none of the States where utilities reduced DSM expenditures in 1993 planned reductions again in 1994 (Figure FE6). New York, a leader in DSM, was among the five States that planned to reduce their DSM expenditures by more than 10 percent in 1994 . Nineteen States showed essentially no change in DSM expenditures for 1994, while the utilities in 26 States and the District of Columbia planned to increase their DSM expenditures by more than 10 percent in 1994.

Over the entire period from 1992 to 1994 , utilities in five States planned to reduce their DSM expenditures by 10 percent or more (ranging from a 32-percent reduction in Michigan to a 15-percent reduction in Missouri, with Delaware, New York, and Oklahoma in between). However, utilities in 10 States (Alaska, Colorado, Georgia, Idaho, Indiana, Kansas, Maryland, New Hampshire, Vermont, and West Virginia) and the District of Columbia planned to more than double their DSM expenditures during this time. ${ }^{6}$

6 For several reasons, readers should be cautious about the State results presented here. First, for all utilities that operate in more than one State, it was assumed that the DSM costs were allocated the same way that retail sales were. However, due to differences in regulatory policies of States, this allocation across States may not be correct. Because of these complexities, energy savings and peakload reductions data are not presented at the State level. 
Figure FE6. U.S. Electric Utility Changes in DSM Program Expenditures by State, 1992 - 1993 and 1993 1994

DSM Expenditures Up More Than $10 \%$ From 1992 to 1993 in 28 States

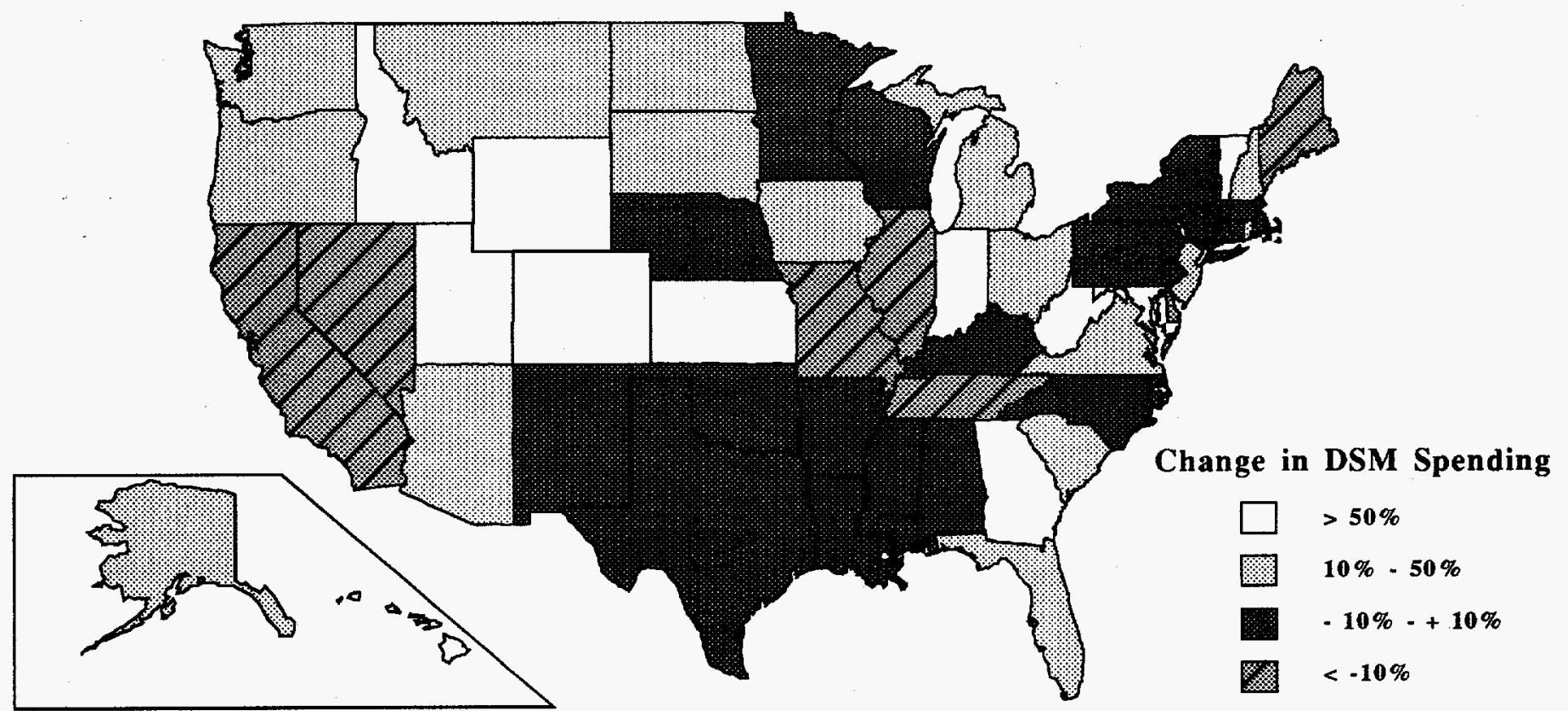

DSM Expenditures Up More Than $10 \%$ From 1993 to 1994 in 27 States

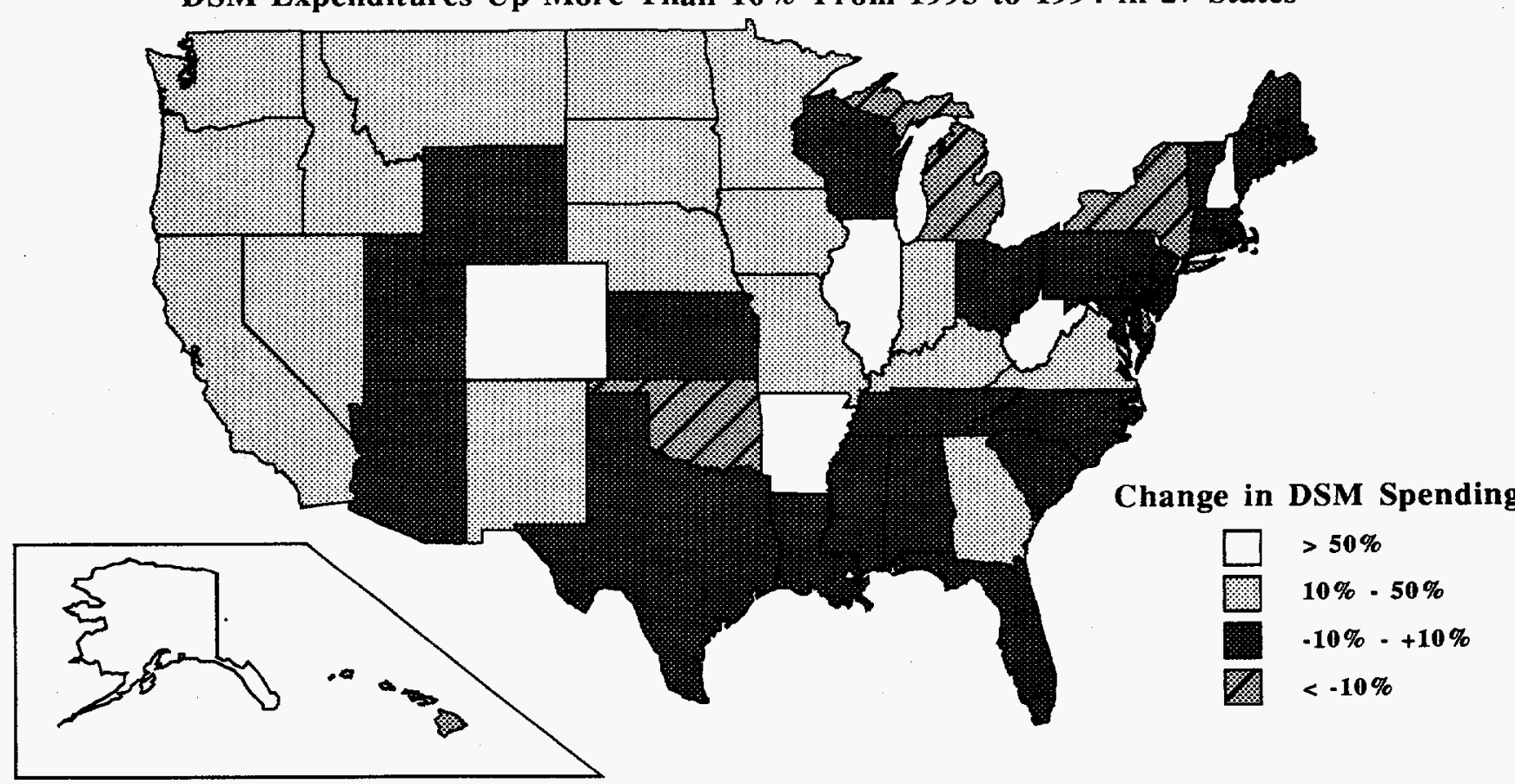

Note: Data for 1994 are projected.

Source: Energy Information Administraion, Form EIA-861, "Annual Electric Utility Report." 


\section{DSM at the Utility Level: 25 Utilities with the Greatest DSM Expenditures}

In 1993, 977 utilities were reported on the Form EIA-861 to EIA as having DSM programs. This discussion will focus on the 25 utilities that had the greatest expenditures on DSM in 1993. These 25 utilities accounted for approximately two-thirds of all utility DSM expenditures in the United States. DSM expenditures for these utilities ranged from $\$ 34$ million for Northern States Power to $\$ 165$ million for the Bonneville Power Administration. Four of these utilities are in California; the remaining 21 are geographically dispersed, providing DSM services in more than 20 States.

Individual utility DSM data were even more varied than regional or State level data for DSM expenditures (Table FE3). No pattern for the changes in expenditures was observed from 1992 to 1993 or from 1993 to 1994 . Of these 25 utilities, 5 decreased their DSM expenditures, and 20 increased expenditures in 1993. The greatest increases were for Bonneville Power Administration ( $+\$ 47$ million), Georgia Power (+\$46 million), Florida Power \& Light $(+\$ 44$ million), and Southern California Edison $(+\$ 41$ million); the greatest decreases occurred at Pacific Gas \& Electric ( $\$ 45$ million) and Niagara Mohawk ( $\$ 24$ million).
For 1994, 9 of these utilities planned to reduce their DSM expenditures and 16 planned to increase spending on DSM. Only Niagara Mohawk and Connecticut Light \& Power reported decreased expenditures for both 1993 and 1994. Increases in expenditures for both 1993 and 1994 were reported by 13 utilities. From 1992 to 1994, 17 of the utilities increased their DSM expenditures, while 8 decreased expenditures. This resulted in a net increase in DSM expenditures for these 25 utilities from 1992 to 1993 and again from 1993 to 1994 , which is consistent with the national totals.

Annual energy savings data for these 25 utilities typically showed increases from 1992 to 1993, and utilities predicted increases from 1993 to 1994 . Only one utility reported decreased energy savings for 1993. Of the 24 utilities reporting growth in energy savings, the most substantial increase was reported by Bonneville Power Administration (1,756 million $\mathrm{kWh}$ ) for 1994.

Potential peakload reductions for these 25 utilities also increased in 1993. Increases of $500 \mathrm{MW}$ or more for potential peakload reductions were reported by Southern California Edison and the Tennessee Valley Authority in the period from 1992 to 1994. Only Boston Edison reported a slight decrease in 1993 and again for 1994 .

Table FE3. Annual Changes in DSM Expenditures for the 25 U.S. Electric Utilities with the Greatest DSM Expenditures, 1992 - 1993 and 1993 - 1994 (Percent)

\begin{tabular}{|c|c|c|}
\hline Utility Name & $\begin{array}{c}\text { Percent Change } \\
1992-1993 \\
\end{array}$ & $\begin{array}{c}\text { Percent Change } \\
1993-1994 \\
\end{array}$ \\
\hline Baltimore Gas and Electric . ................ & 120 & 10 \\
\hline Bonneville Power Administration $\ldots \ldots \ldots \ldots \ldots$ & 40 & 31 \\
\hline Boston Edison $\ldots \ldots \ldots \ldots \ldots \ldots \ldots \ldots \ldots$ & 2 & 3 \\
\hline Carolina Power and Light $\ldots \ldots \ldots \ldots \ldots \ldots$ & -31 & 5 \\
\hline Connecticut Light and Power $\ldots \ldots \ldots \ldots \ldots \ldots$ & -9 & -3 \\
\hline Consolidated Edison $\ldots \ldots \ldots \ldots \ldots \ldots \ldots \ldots$ & 8 & 0 \\
\hline Consumers Power . ................... & 60 & -72 \\
\hline$\ldots \ldots \ldots \ldots \ldots$ & 15 & -7 \\
\hline Florida Power Corporation . . . . . . . . . . . . . & 34 & 2 \\
\hline Florida Power and Light . . . . . & 45 & 11 \\
\hline Georgia Power ......... & 676 & 13 \\
\hline Massachusetts Electric $\ldots \ldots \ldots \ldots \ldots \ldots \ldots$ & 4 & 40 \\
\hline N.Y. State Electric and Gas $\ldots \ldots \ldots \ldots \ldots \ldots$ & 28 & -65 \\
\hline Niagara Mohawk Power . ................. & -36 & -2 \\
\hline Northern States Power . . . . . . ............ & 12 & 46 \\
\hline Pacific Gas and Electric $\ldots \ldots \ldots \ldots \ldots \ldots$ & -25 & 27 \\
\hline Pacificorp $\ldots \ldots \ldots \ldots \ldots \ldots \ldots \ldots \ldots \ldots$ & 83 & 1 \\
\hline Potomac Electric Power Co. ............... & 81 & 32 \\
\hline PSI Energy $\ldots \ldots \ldots \ldots \ldots$ & 97 & -4 \\
\hline Public Service Electric and Gas $\ldots \ldots \ldots \ldots \ldots$ & 15 & 17 \\
\hline Puget Sound Power and Light . & 10 & -26 \\
\hline Sacramento Municipal Utility District & -10 & 54 \\
\hline Southern California Edison . ....... & 47 & 7 \\
\hline Tennessee Valley Authority . . & 9 & 9 \\
\hline Wisconsin Electric Power & 7 & -17 \\
\hline
\end{tabular}

Note: Data for 1994 are projected.

Source: Energy Information Administration, Form EIA-861, "Annual Electric Utility Report." 


\section{Forecast of DSM}

The perception and the reality of competition in the electric utility industry are increasing. Analysts that believe competition will focus narrowly on the price of electricity expect that traditional DSM programs where utilities implement diverse programs in which all utility customers pay for most of the DSM investments will diminish. They expect that with the pressure to reduce prices, utilities and their regulators will focus on programs that produce short-term profits in which only the participants pay for the services received. Analysts that predict competition will encompass a broad range of energy services also predict that environmental quality will continue to be an important issue for society and for utility customers. ${ }^{7}$ Both projections would produce a different future for DSM. It is expected that utilities and other providers (e.g., energy service companies) will offer a variety of services, including energy efficiency and load management programs. Some of these analysts also think that State regulators will insist that all electricity users pay for DSM programs that provide societal benefits even if these programs raise electricity prices slightly.

The data that utilities submitted to EIA for the 1993 reporting year indicate that the effects of DSM programs are increasing, although DSM expenditures are increasing at a lesser rate than in the past. Thus, predictions that deregulation may lead to the demise of DSM are premature. Nationwide, DSM expenditures and effects increased from 1992 to 1993, and are projected to increase again from 1993 to 1994 and in 1998. However, the rate of increase for expenditures, although not for energy savings, is projected to decline.

Changes in the electric power industry within utilities, State regulatory commissions and the Federal Energy Regulatory Commission, and the financial community suggest that utilities will generally seek to minimize the adverse rate impacts of DSM programs. In some cases, this will involve reducing DSM budgets; in other cases, it may involve shifting program priorities away from energy efficiency and toward peakload reduction. In general, utilities are likely to focus their DSM programs more on customer service and customer productivity, and less on energy and capacity resources. Time will show whether DSM programs continue to grow, as predicted by utilities in 1993, or whether DSM will decline or be transformed by the forces of competition and industry restructuring. Following are some observations and speculations on utility DSM programs:

- In 1993, utilities planned for continued increases in DSM spending and effects. Expenditures for energy efficiency programs continued to dominate in 1993, as in 1992, and are projected to continue in 1994, although the rate of growth is declining for DSM expenditures.

- Actual utility DSM expenditures in 1993 were almost 7 percent below the levels estimated on the 1992 . Form EIA-861 submissions. The actual 1993 energy savings data were about the same as those estimated on the 1992 forms, and the actual 1993 peakload reduction was higher than that estimated on the 1992 forms.

- The 1994 data are anticipated to show lower DSM spending and activities for 1994 than the estimates reported on the 1993 Form EIA-861 submissions. Also, DSM spending for 1998 is expected to fall below the forecasts made on the 1993 forms. Recent reports from utilities in some States, especially California and New York, suggest further reductions in DSM spending.

- Utilities will likely and increasingly require participating customers to pay for the DSM services that they receive. Thus, utilities may reduce rebates and emphasize loans and other financing mechanisms that do not require contributions from nonparticipating customers. Utilities will likely develop improved market segmentation and marketing approaches that will allow them to maintain program participation levels at lower costs to all ratepayers.

- Utilities may focus their view of DSM on customer service (e.g., productivity and comfort improvements) and reduce their emphasis on DSM as an energy and capacity resource. In particular, utilities will downplay energy savings, primarily because of the revenue lost by such programs.

Recent trade media reports indicate that some utilities plan to expand their DSM programs. Utilities attribute such increases to DSM cost-effectiveness, compared with supply-side resources such as capacity additions, or to initiatives to improve customer service. Other utilities plan to reduce the rate of growth for DSM expenditures. Some of the greatest reductions in expenditures for DSM programs appear to be at utilities with rates (cents per $\mathrm{kWh}$ or $\mathrm{kW}$ ) that are high relative to their marginal production costs. Reduced sales for these utilities would have an adverse effect on revenue. Some of the greatest reductions are in California, where a proceeding for retail access is pending. California utilities have argued that DSM should continue under retail access. The treatment of DSM under increased competition and retail access, however, remains uncertain. DSM spending has been affected by utility efforts to prepare for competition by reducing costs company-wide.

7 DSM contributes to an enhanced environment through reducing the consumption of fuels and construction of new power plants. 


\section{Conclusion}

The effects of increased competition in the electric power industry on DSM are complex and their overall impacts uncertain. Some of the impacts could reduce the growth of DSM. If increased competition leads to increased generating unit efficiency and availability, the need for new resources could be deferred and less DSM may be cost effective. If DSM significantly reduces consumption, market prices for generation could be reduced, leading to a conflict of interests between DSM and ownership of deregulated generation. This conflict is not present under a noncompetitive, regulated environment where State utility commissions permit utilities to recover costs and lost revenue associated with reduced sales in the rate base.

In a competitive market, prices are likely to be unbundled with the energy component of prices for generation services moving toward marginal cost. Rate design changes could have three effects on DSM programs. First, according to analyses conducted by some utilities, reduced volumetric charges could significantly reduce or eliminate negative rate impacts from DSM programs that reduce sales. Second, as the energy component of rates falls toward marginal costs, incentives paid to customers, vendors, and manufacturers could become increasingly important to encourage DSM. This effect could partially offset a trend toward reducing incentives to lower utility costs. Finally, under retail transmission access, unbundled charges for all transmission and/or distribution customers could be required to support utility DSM.

Other impacts could have a positive effect on DSM. Increased competition could prompt the expanded use of time-of-use and real-time pricing, as well as other load-management techniques. Additional data on patterns of customer energy use would be gathered to implement time- and location-specific pricing options. Assuming a constructive resolution of confidentiality and proprietary access questions, such information could help customers make more efficient energy choices.

The U.S. Department of Energy is sponsoring research to learn more about utility DSM plans and the reasons for possible changes in DSM program size and direction. This project, conducted by the Oak Ridge National Laboratory, the American Council for Energy-Efficient Economy, and the Electric Power Research Institute, involves detailed interviews with personnel from about 30 utilities and 20 State regulatory commissions. Approximately half the sample will consist of utilities and States where DSM is growing and the other half will consist of utilities and States where DSM is declining. The project will be completed in the summer of 1995. 


\section{Evaluation and Verification of Demand-Side Management Programs}

\section{Introduction}

In 1992, Congress mandated the Energy Information Administration (EIA) to collect information on electric utility demand-side management (DSM) programs, including estimates of energy savings resulting from such programs and whether such savings estimates "were verified" (15 U.S.C. 2790a, as amended by ?171(b)(k)(2) of the 1992 Energy Policy Act). Since 1989 , utilities have reported the peakload reductions and energy savings achieved as a result of their DSM efforts to the Energy Information Administration (EIA) on the Form EIA-861, "Annual Electric Utility Report," an annual census of U.S. electric utilities. Utilities with sales to ultimate consumers or sales for resale greater than or equal to $120,000 \mathrm{MWh}$ must report their annual peakload reductions, energy savings, and costs attributable to DSM programs. ${ }^{1}$ Pursuant to the congressional mandate, EIA included on Form EIA-861 for 1993 questions regarding whether reported DSM energy effects and peakload reductions undergo a verification process, the methodologies used in verification, and the purposes for which verification was carried out. In 1993, 545 large utilities with DSM programs were reported on the Form EIA-861. ${ }^{2}$ EIA's data provide the most comprehensive available information on the energy savings and peakload reductions achieved by U.S. electric utility DSM programs.

This article ${ }^{3}$ presents a preliminary assessment of whether utilities evaluate or verify their energy savings and peakload reductions and the extent to which the evaluation results confirm savings reported on the Form EIA-861. The fifty utilities that reported the largest incremental energy savings and peakload reductions were contacted to obtain more detailed data on their evaluation and verification procedures. Twenty-five utilities agreed to provide additional information regarding their procedures. This information, together with the data gathered on the 1993 Form EIA-861, provide the basis for this assessment. The study examined evaluation and verification activity and spending at the utilities, peakload reductions and energy effects that have been the subject of detailed evaluation studies, and, where adequate data were available, the extent to which evaluation results confirm savings reported on Form EIA-861.

Before discussing the results of this assessment study, a background for the development of evaluation and verification activities for utility DSM programs is presented.

\section{Background}

Electric utilities have increased DSM programs and expenditures rapidly in recent years. This can be attributed to an increased reliance on DSM as an alternative resource, thus deferring the need for additional generation capacity, and increased regulatory requirements that DSM be considered in utilities' Integrated Resource Planning. The reliance on DSM as a resource and the growth in energy savings, peakload reductions, and spending has increased attention on the extent to which DSM programs are achieving projected energy savings and peakload reductions.

In 1991, S. Nadel and K. Keating identified a gap between engineering estimates of savings and the results of impact evaluations for certain types of DSM programs. Examining the results of 42 impact evaluations, some of the first impact evaluations completed for large-scale utility DSM programs, Nadel and Keating found that projections of savings based on engineering estimates significantly exceeded verified savings from subsequent program evaluations. This was true particularly for residential retrofit programs, residential and commercial lighting programs directed at small customers, and programs involving the installation of low-flow shower heads. For residential appliance programs, new construction programs, and multiple measure commercial and industrial retrofit programs directed at large customers, they found that engineering estimates and impact evaluation results were, on average, comparable. The disparity between engineering estimates and evaluation results for some

1 Utilities with sales to ultimate consumers and sales for resale less than 120,000 MWh only report incremental energy savings and peakload reductions, and the total costs of their DSM programs.

2 Some utilities with DSM programs were reported on another utility's Form EIA-861; however, the forms received in 1993 represent the DSM activities of 545 large utilities. Consequently, the evaluation and verification data was reported only by those utilities that actually filled out the Form EIA-861. ated.

3 Paul Centolella's support and contribution to the Electric Operating and Financial Data Branch in preparing this article are greatly appreci- 
DSM programs contributed to a spirited debate regarding the benefits of DSM. ${ }^{4}$

The most detailed comparative analysis of DSM program evaluations completed to date calculated a total utility and customer cost of saved energy for utility commercial lighting programs that was somewhat higher than some earlier estimates, but found that all of the programs studied were cost-effective. ${ }^{5}$ Other analysts have noted variance in the results of DSM programs and sought to identify characteristics of successful programs. ${ }^{6}$ The disparity between engineering estimates and evaluation results identified by Nadel and Keating has led DSM professionals to emphasize program evaluation, integration of evaluation results into estimates of program performance, and reduction in the disparity. More recent reviews of utility evaluations show greater comparability between initial estimates of program performance and the results of detailed impact evaluation.?

Most utilities with large DSM programs evaluate their programs to assess whether they are achieving projected peakload reductions or changes in energy usage, meeting customer needs, and/or are effective and efficient in their design, marketing, and implementation. ${ }^{8}$ Evaluation involves load, behavioral, market, organizational, or other research studies designed to provide useful information to program planners, regulators, or other decisionmakers.

\section{History of Energy Program Evaluation}

The application of the tools of social science research and statistics to the assessment of public programs dates back to the evaluation of agricultural programs in the 1930's. During the 1960 's, program evaluation techniques were applied to government social programs. In the late 1970's, program evaluation methods began to be adapted to Federal Government energy programs, such as the State Energy Conservation Program and the Energy Extension Service.

Over the years, considerable technical literature has developed regarding the practice of program evalu- ation, including the journal and publications of the American Evaluation Association. In 1984, Argonne National Laboratory initiated a series of conferences on energy program evaluation. The conference was repeated in 1985, 1987, 1989, 1991, and 1993. Measurement and evaluation also has been a major topic at the biennial American Council for an Energy Efficient Economy Summer Studies on Energy Efficiency in Buildings, the National Demand-Side Management Conferences, and a variety of other DSM and evaluation professional conferences.

As utility DSM programs grew in the late 1980's, evaluation techniques began to be applied to develop precise and reliable estimates of the exact size and timing of peakload reductions. Methodologies changed from an earlier reliance on primarily survey research to quasi-experimental designs, including controlgroups to allow attribution of program effects. Program impacts are now commonly measured through statistical analysis of customer bills and specialized metering studies. As the role that DSM has played in utility resource acquisition has grown, so has the sophistication of DSM program evaluation. ${ }^{9}$

The role of evaluation also has been changed by the development of State ratemaking practices that either:

- Provide economic incentives to utilities based upon performance of their DSM programs or

- Authorize utilities to recover, through a deferral or rate adjustment mechanism, lost contributions to the recovery of their fixed costs that result from sales reductions attributed to DSM programs.

Regulators in 29 States and the District of Columbia have adopted some form of DSM performance incentive and/or net lost revenue adjustment (Figure FE1).${ }^{10}$ Since projected or actual energy savings and peakload reductions from DSM programs began to affect utility rates, State public utility commissions have started to require and review evaluations of program performance. Regulatory scrutiny has led evaluators to emphasize quantitative savings estimates and improvements in the precision and confidence levels for evaluation results.

4 P. Joscow and D. Marron, "What does a Negawatt Really Cost: Evidence From Utility Conservation Programs," Energy Journal, Vol. 13, No. 4 (1992). A. Lovins, "Apples, Oranges, and Horned Toads: Is the Joscow \& Marron Critique of Electric Efficiency Cost Valid?" The Electricity Journal (May 1994), p. 33.

5 J. Eto, E. Vine, L. Shown, R. Sonnenblick, and C. Payne, The Cost and Performance of Utility Commercial Lighting Programs, Lawrence Berkeley Laboratory, LBL-34967 (May 1994).

6 T. Flanagan and J. Weintraub, "The Most Successful DSM Programs in North America," The Electricity Journal (May 1993), p. 53. M. O'Neil, "DSM: It's a Contact Sport." The Electricity Journal (May 1993), p. 53. S. Nadel, M. Pye, and J. Jordan, Achieving High Participation Rates: Lessons Taught By Successful DSM Programs, American Council for an Energy Efficient Economy (January 1994).

7 See for example, M. Brown and P. Mihlmester, Summary of California DSM Impact Evaluation Studies, ORNL/CON-403 (October 1994).

8 For a general introduction to DSM program evaluation, see: M. McRae, et.al., DSM Evaluation - Six Steps for Assessing Programs, Electric Power Research Institute, CU-6999 (March 1992).

9 For a further discussion of changing directions in evaluation practice, see: M. Kushler, K. Keating, J. Schlegel, and E. Vine, "The Purpose, Practice, and Profession of DSM Evaluation: Current Trends, Future Challenges," Proceedings of the ACEEE 1992 Summer Studies on Energy Efficiency in Buildings (August 1992), p. 7.1.

10 M. Reid, J. Brown, and J. Deem, Incentives for Demand-Side Management, 3rd Ed., National Association of Regulatory Utility Commissioners (October 1993). See also, P. Centolella, S. Mitnick, B. Barkovich, and D. Boonin, Cost Allocation for Electric Utility Conservation and Load Management Programs, National Association of Regulatory Utility Commissioners (February 1993). Note: some jurisdictions that do not have lost revenue recovery mechanisms have adopted revenue decoupling approaches that do not rely on the results of DSM program evaluations. 


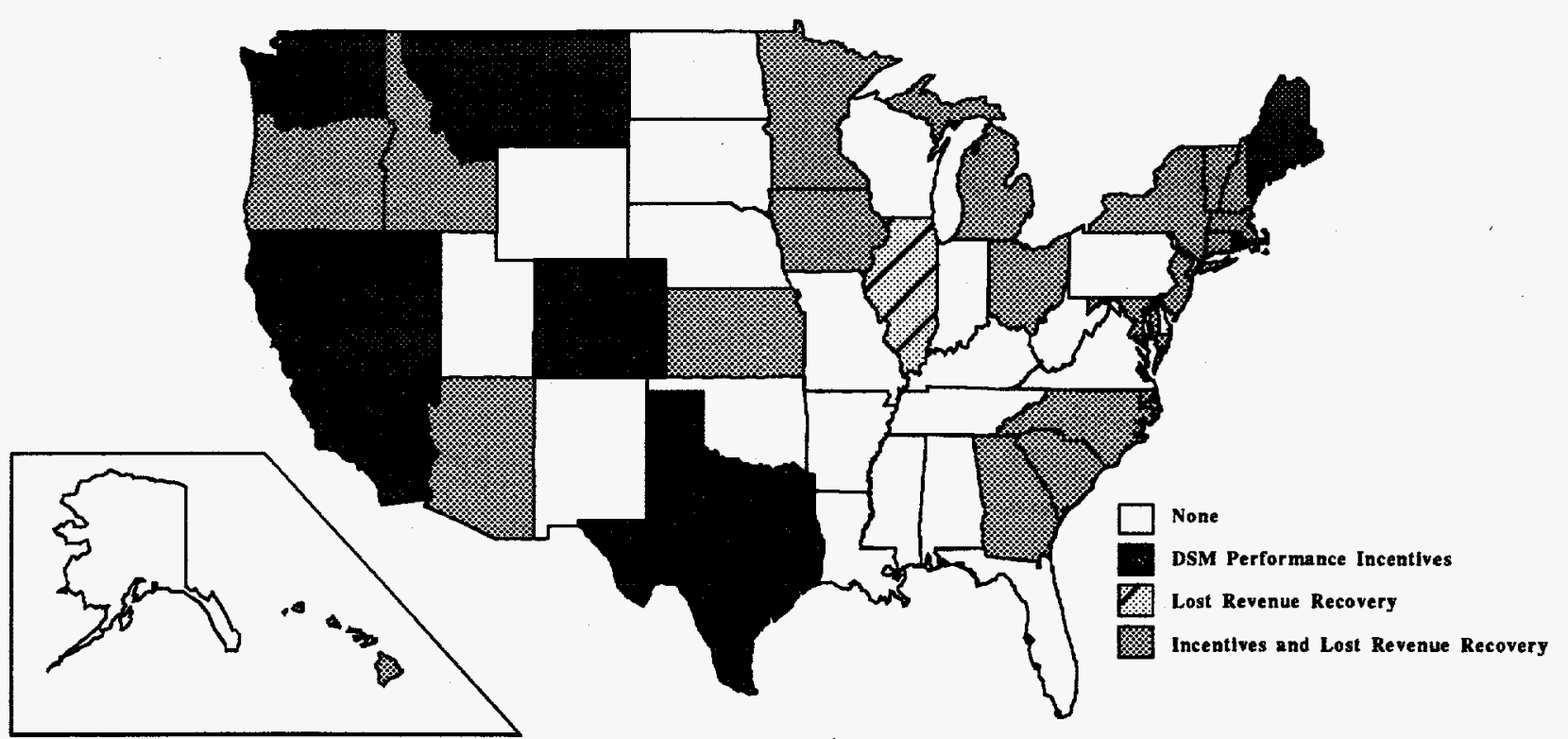

Source: Office of Coal, Nuclear, Electric and Alternate Fuels, DSM Verification Study of 25 Selected U.S. Electric Utilities.

\section{Purposes of Utility DSM Program Evaluation}

DSM program evaluations may be undertaken to meet one or more of the following basic objectives:

- Measurement of program impacts, including the measurement of energy effects, peakload reductions, changes in load shape, free ridership, contagion, persistence of savings, and the cost of measure implementation

- Identification of program improvements, including issues related to program operation, design, or organizational development; barriers to customer participation; and reasons for measures performing differently from expectations

- Description and characterization of program operation and effects, including how the program works, the market segments that have been reached, the market that remains, the interaction between DSM measures and customer behavior, or why program results occurred.

Verification of quantitative savings estimates is made primarily through impact evaluations. DSM program impact evaluation is an examination of the effects of a program, including the quantitative documentation of the program's costs and benefits, the rate of participation and measure adoption, the performance of the DSM technologies, and the energy and load impacts. ${ }^{11}$

\section{Verification Defined}

Generally, almost all impact evaluations are a process of verification. Typically, such studies are structured to compare changes in energy use in facilities that are selected by utilities to tabulate participation with changes in energy use in non-participating facilities. In this sense, impact evaluations verify the credibility of the utility's estimate of savings based on its tracking of program participation.

Verification also has come to have the more specialized meaning of meeting specified standards of proof for securing some economic benefit related to measured DSM program performance. Verification, in this sense, refers to confirming the achievement of savings as a contractual condition for payments to an energy service company or contractor, for purposes of granting the utility a shareholder incentive or the

11 For a description of impact evaluation methodologies and practices, see: D. Violette, M. Ozog, M. Keneipp, F. Stern, and P. Hanser, Impact Evaluation of DSM Programs, Vol I: A Guide to Current Practice, Electric Power Research Institute, CU-7179, Vol. I (February 1991). D. Violette, F. Stern, and M. Ozog, Impact Evaluation of DSM Programs, Vol. II: Case Studies and Applications (Revision I), Electric Power Research Institute, CU-7179, Vol. II, Revision I (November 1992). 
recovery of lost contributions to the margin associated with lower sales, ${ }^{12}$ or for the award of conservation and renewable energy allowances under the 1990 Clean Air Act Amendments. ${ }^{13}$

In this more specialized context, verification requirements can change the objectives and form of evaluation studies. Verification in this context cannot be an academic exercise directed at achieving statistically significant results, regardless of the complexity or time involved. Verification applies contractual or regulatory standards and places premiums on the clarity and reasonableness of the analysis, timely completion within a defined schedule, and a need for closure on a specific quantitative result. Protocols for undertaking such verification studies may be described in public utility commission approved verification plans or by specific contractual agreement. For example, the California Public Utilities Commission adopted detailed standards and procedures for the verification of program costs and benefits for purposes of utility claims for shareholder earning incentives (Part I), that are distinguished from the more general measurement agreements covering evaluation for resource planning purposes (Part II). ${ }^{14}$ Such protocols may specify a degree of precision and confidence level that must be achieved by the results of the verification study. ${ }^{15}$

\section{Evaluation and Verification Methodologies}

The objective of impact evaluation is to measure a change in energy use as a result of intervention in the market by a utility's DSM program. This objective is different from that of many other load research activities, including energy and demand forecasting, equipment saturation studies, and load shape characterization, that are designed to determine levels of energy consumption. Because impact evaluations focus on measuring changes in customer behavior and energy consumption as a result of a specific utility action, they can rely on methodologies that are experimental in nature. The key to such approaches is to define a baseline of what consumption would have been in the absence of the utility's DSM program. Control groups of non-participants are commonly utilized, but care must be taken to address self-selection bias and free-ridership (customers who would have installed a DSM measure even in the absence of the utility's program), as well as spillover or contagion effects of the program on non-participating customers and the adoption by participants of energy manage- ment measures not included in the program. The objective is to measure impacts only due to utilitysponsored DSM programs.

Development of an efficient and effective evaluation requires planning that often begins before a DSM program is implemented, a clear understanding of the objectives of the evaluation and how those objectives are to be measured, and accepted definitions of key terms. ${ }^{16}$

To identify net program impacts, evaluators often use a combination of engineering, statistical, metering, and survey approaches. Methodologies that are used to measure energy effects and/or peakload reductions in impact evaluations include:

- Engineering Methodologies: Engineering methods are used primarily for evaluating new construction programs, estimating coincident peakload savings using measured energy savings and load shape information, and developing engineering estimates for use in conjunction with statistical analysis.

- Statistical Analysis of Customer Billing Data: Statistical analysis of billing data covers a variety of methodologies from simple pre-installation/postinstallation comparison of billing data to complex regression models. Billing analysis typically compares consumption data before and after measure installation for a sample of participants with changes in energy consumption for a nonparticipant control group over a comparable time period. Such methodologies commonly normalize consumption data to take into account weather, and, in some cases, other factors such as economic conditions or energy prices. Some analyses utilize multi-variate regression techniques to increase precision by explaining the variability in savings due to factors such as weather, economic conditions, customer attitudes, or independent engineering estimates. Conditional demand models are one commonly used form of multi-variate regression analysis that rely on the fact that total consumption within a facility may be broken down by its end uses and the fact that evaluation is designed to measure only changes in consumption as a result of the installation of DSM measures and to estirnate changes in end use loads attributable to specific conservation measures. ${ }^{17}$ Billing analysis is particularly useful for estimating net energy savings from a program in that it captures both technological and behavioral effects on participant consumption.

M. Reid, et.al. (1993)

1342 U.S.C. ?7651(c)(f), as amended by Public Law 101-549. U.S. Environmental Protection Agency, The User's Guide to the Conservation Verification Protocols, EPA 430-B-93-002 (April 1993).

14 California Public Utilities Commission, Protocols and Procedures for Verification of Costs, Benefits, and Shareholder Earnings from DemandSide Management Programs (July 1994).

15 Ibid. U.S. Environmental Protection Agency (1993).

16 A significant contribution to the definition of common terminology and reporting formats occurred in 1991 with the publication of E. Hirst and C. Sabo, Electric-Utility DSM Programs: Terminology and Reporting Formats, Oak Ridge National Laboratory, ORNL/CON-337 (October 1991).

17 M. Parti and C. Parti, "The Total and Appliance-Specific Demand for Energy in the Residential Sector," Bell Journal of Economics, Vol. 11, No. 1 (Spring 1980), pp. 309-321. M. Parti and C. Parti, "Estimating the Consumption Impact of a Residential Conservation Program," Proceedings of the 1992 ACEEE Summer Study on Energy Efficiency in Buildings (August 1992), p. 4.177. 
- Measurement and Metering: A range of direct measurement and metering approaches can be employed, including: measurements of hours of usage for lighting or equipment, spot metering to measure instantaneous loads, whole facility metering to provide a time-differentiated picture of energy consumption, and end-use metering for specific equipment or processes. Metering has the major advantage of providing an accurate, high resolution measurement that can be focused on the end use(s) of interest. Since metered data are differentiated by time period, it can be used to estimate reductions in coincident peak load and changes in load shape.

- Site Visits and Surveys: Site visits can be utilized to verify the installation of measures and improve tracking systems for DSM data. They also may be used to gather information that will be utilized in engineering or statistical analysis. Surveys may be used in impact evaluation to help estimate free ridership and contagion, evaluate factors that influence customer participation decisions, segment potential markets for the DSM program, or collect data for use in statistical analysis.

- Combination Approaches: The combination of multiple evaluation methodologies can increase the precision or reduce the sample size required for a given level of precision by explaining some of the variance in savings based on other available information. There are several approaches that utilize multiple methods including:

- Triangulation compares the results of more than one method to estimate program impacts. Results of each method are compared to one another to form judgements regarding the quality and reliability of each estimate. ${ }^{18}$ For example, triangulation may be used to compare estimates of free ridership from survey data and participation models.

- Statistically adjusted engineering estimates combine engineering estimates with statistical analysis of billing or metered data. In this approach, engineering estimates are used as independent variables in a regression equation, in the place of a dummy variable for participation. ${ }^{19}$

- Leveraging data through the use of stratification and ratio estimation procedures can increase the precision of results by using additional information (such as square feet of conditioned space) regarding the population of participants to adjust estimated savings. ${ }^{20}$

- Bayesian methods have been proposed as a systematic approach to update and adjust prior information in a new evaluation. Information from previous engineering estimates, evaluations, or expert judgement could be used as inputs in a Bayesian estimation framework. ${ }^{21}$

Such methodologies are used to estimate both initial savings as a result of DSM programs and the persistence of savings over time.

\section{Verification and Form ElA-861 Peakload Reduction and Energy Effects Data}

Because EIA relies on a survey of electric utilities to gather peakload reduction and energy effects data, it would be difficult for EIA to standardize utility program evaluation practices or protocols. However, it is possible to examine the extent to which data reported to EIA may have been subject to evaluation under State requirements or the requirements of other Federal legislation, such as the requirement to use verified data in applying for conservation and renewable energy reserve allowances under the $\mathbf{1 9 9 0}$ Clean Air Act Amendments.

On the 1993 Form EIA-861, utilities were asked, "Do your Energy Effects and Peak Reductions undergo a verification process?" ${ }^{22}$ Among the large utilities ${ }^{23}, 380$ responded to this question. ${ }^{24}$ Of those who responded, 238 indicated that the data reported to EIA was subject to some form of verification process. Additionally, utilities were asked to identify the primary and secondary methodologies (Figure FE2) they used to verify energy effects and peakload reductions from the following choices:

- Verfication of Installation

- Stipulated Savings

- Measurement of Hours of Usage

- Billing Data Metering

- Whole Building Metering

- End-Use Metering

- Site-Specific Engineering Analysis

18 R. Ridge, "Triangulation and Budget Constraints in the Evaluation of Large Non-Residential Demand-Side Management Programs," Proceedings of the 1993 International Energy Program Evaluation Conference (August 1993), p. 251.

19 D. Violette, R. Brakken, A. Schon, and J. Greer, "Statistically Adjusted Engineering Estimates: What Can the Evaluation Analyst Do About the Engineering Side of the Analysis?," Proceedings of the 1993 International Energy Program Evaluation Conference (August 1993 ), p. 656.

20 D. Violette, "New Directions in Impact Evaluation Methods," 1993 International Energy Program Evaluation Conference Workshop, Association of Demand-Side Management Professionals (August 1993).

21 D. Violette, "Analyzing Data," Handbook of Evaluation of Utility DSM Programs, E. Hirst and J. Reed, ed., Oak Ridge National Laboratory (1991), p. 51-72.

22 Energy Information Administration Form EIA-861, "Annual Electric Utility Report," p. 6. As used in this form, the terms "Energy Effects" and "Peak Reductions" are specifically defined and refer to the data reported on pages 4 and 5 of that form.

${ }^{23}$ Large utilities are those with sales to ultimate consumers or sales for resale greater or equal to $120,000 \mathrm{MWh}$.

24 Although 545 large utilities were represented on the 1993 Form EIA-861, some utilities' DSM activities are reported by another utility; hence, not all 545 large utilities actually filled out the DSM information requested on Form EIA-861 and had the opportunity to answer this question. The actual total who did answer this question was 380 . 
- Statistically Adjusted Engineering Analysis

- Other

Finally, utilities were asked to identify the primary and secondary purposes for undertaking verification analysis. Choices provided in the question included: internal utility evaluation requirement, State or Federal regulatory agency requirement, EPA conservation verification protocol or environmental requirement, terms of a performance contract, or other. Half of the large utilities, 119 of 238, indicated they are engaged in verification of DSM programs to meet a State or Federal regulatory requirement.
The responses to the evaluation and verification information on the Form EIA-861 by those 380 utilities indicate that most utilities are undertaking some form of program verification. It is not, however, possible to draw a direct inference from these responses regarding the overall accuracy of data recorded on Form EIA-861. A review of the evaluation and verification procedures of the 25 utilities that agreed to provide EIA with additional information on the procedures and results of impact evaluations will provide a preliminary assessment of the extent to which EIA data are consistent with the results of utility evaluation and verification studies.

\section{Figure FE2. U.S. Electric Utility Primary Methodologies for Verification of DSM Energy Effects and Peakload Reductions by Number of Utilities, 1993}

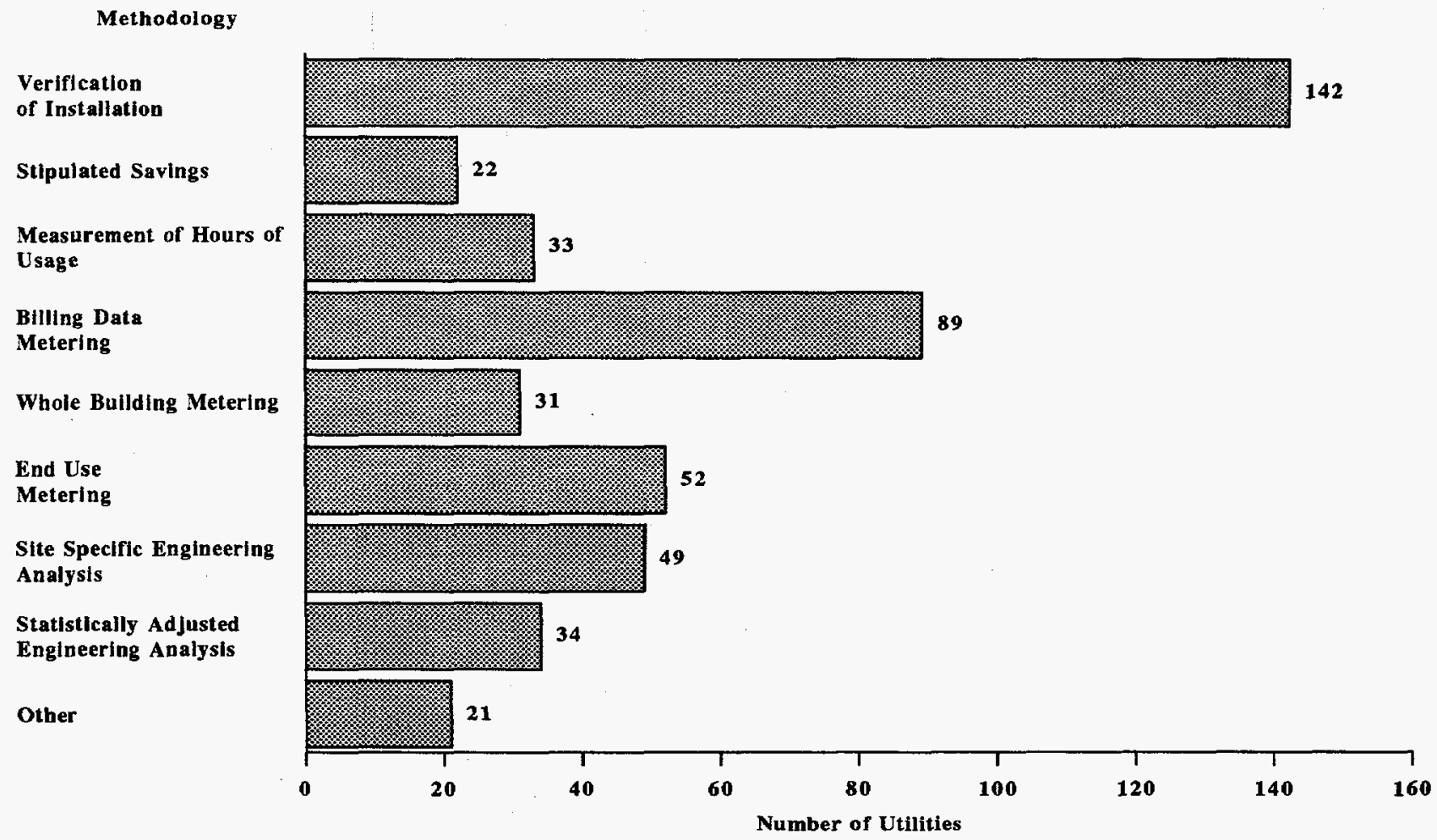

Note: Individual utilities may use more than one primary methodology.

Source: Energy Information Administration, Form EIA-861, "Annual Electric Utility Report."

\section{A Study Approach}

The fifty utilities that reported the largest energy savings and peakload reductions for 1993 on the Form EIA-861 were asked to provide information, if readily available, on their evaluation processes. Twenty-five companies, ${ }^{25}$ representing 29 Form EIA-861 respondents, given holding companies and recent mergers, agreed to provide information on their evaluation processes. Of interest were the number of evaluations performed during the years 1990 through 1994, utility spending on DSM program evaluation for the years 1990 through 1994, the length of time between close of the program year and the completion of impact evaluations, the consideration of prior year evaluation results in estimating DSM impacts, State utility commission requirements for DSM program evaluation, and the proportion of 1993 total energy effects and peakload reductions associated with DSM programs for which the utility had conducted impact evaluations. Additionally, the utilities were asked to identify the four individual programs that made the greatest contribution to 1993 incremental energy savings and the four programs that made the greatest contribution to incremental peakload reductions, including information on the methodologies used in the evaluation of these programs.

${ }_{25}$ The time and effort of those utilities that participated in this study, and in many cases provided copies of impact evaluation reports, are sincerely appreciated. 


\section{Study Results}

A review of the 25 utilities' responses to the study show that expenditures on evaluation processes have increased since 1990. Moreover, for most of these utilities a large fraction of the reported energy and peakload savings are from programs that have been subject to impact evaluations. In the following sections, the scope of evaluation activity and its implications for consistency of the reported peakload reductions and energy savings on the Form EIA-861 with the results of the impact evaluations are evaluated.

\section{Number and Type of Evaluations Performed}

The 25 utilities that provided information on their evaluation processes identified 336 separate impact evaluations of DSM programs. Of the completed evaluations, 155 covered DSM programs for the residential sector, and 191 addressed programs available to commercial and industrial customers. ${ }^{26}$

Although the number of evaluation studies was large, it should be noted that they were not evenly distributed among the participating utilities. Six utilities, each of which had completed more than 20 impact evaluations, accounted for 225 of the 336 studies identified. Indeed, one utility had completed 51 impact evaluations, another 48 , and a third 45 such studies. In each of these cases, it appeared that the utility had prepared separate evaluations for most or all of its DSM programs for each program year, 1990 through 1994. Other utilities had fewer DSM programs or had completed one comprehensive evaluation for each program covering the period without performing repeated annual evaluations (Figure FE3).

Most of the utilities that participated in the study had completed multiple impact evaluations, but five companies had not completed any impact evaluation studies during the years 1990 through 1994. Of these five utilities, three were located in States that have no current requirement that utilities perform DSM program evaluations. The remaining two were in States where an evaluation requirement had been adopted by the State public utility commission in June 1994 and in December 1994, respectively. The utility which became subject to an evaluation requirement in June 1994 said that evaluation efforts were underway for two of its programs. The remaining four utilities did not have any evaluations in progress. The utilities that had not undertaken any impact evaluations had lower 1993 incremental energy savings as a percentage of annual sales, when compared with utilities that had completed one or more evaluations. The utilities that had not undertaken evaluations to a much greater degree had concentrated their DSM programs on achieving peakload reductions, as opposed to energy savings.

Figure FE3. Number of Program Evaluations Performed by 25 U.S. Electric Utilities, 1990 through 1994

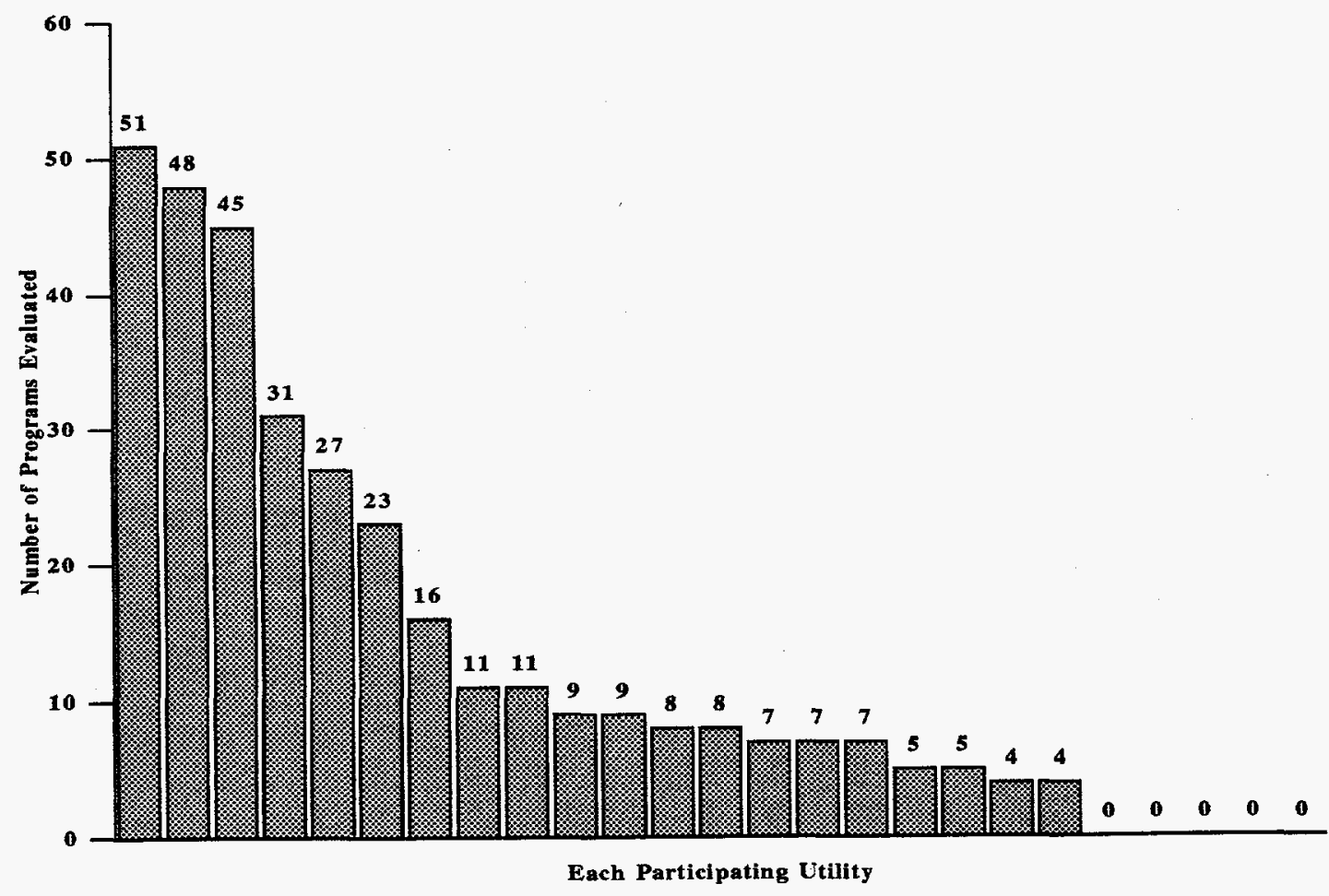

Source: Offlce of Coal, Nuclear, Electric and Alternate Fuels, DSM Veriflcation Study of 25 Selected U.S. Electric Utilities.

26 Eleven program evaluations addressed programs that were available to residential, commercial, and industrial customers. 
Comparing the evaluations identified in the study to the types of energy efficiency end uses and sectors for which the utilities reported DSM activities, residential load control programs were evaluated more frequently and programs aimed at industrial process uses were evaluated less frequently than other programs.

\section{Evaluation of Spending}

Information on DSM evaluation cost was provided by 20 of the 25 utilities in the study. In 1990, only 6 of the 20 utilities reported evaluation spending, for a total of $\$ 3.1$ million. The number of utilities increased to 11 in 1991, 18 in 1992, 19 in 1993, and 20 in 1994 when these utilities spent over $\$ 24$ million (Figure FE4). Spending by these 20 utilities peaked in 1992 at nearly $\$ 28.5$ million. The 1992 peak in evaluation spending is partially attributable to large initial evaluation expenditures by three utilities that began evaluation activities in that year. These three utilities spent $\$ 4.9$ million, $\$ 2.3$ million, and $\$ 3.4$ million, respec- tively in 1992, their initial year of evaluation spending, and reduced spending on evaluation in 1993 to $\$ 2.9$ million, $\$ .3$ million, and $\$ 1.1$ million, respectively. The growth in evaluation spending is in part attributable to the increased number of utilities engaged in evaluation.

Another perspective on DSM evaluation spending by these 20 utilities considers the average amount spent by these utilities in the period from 1990 through 1994 (Figure FE5). Evaluation spending as a percentage of total DSM spending declined more rapidly in 1993 and 1994 than in 1990 through 1992, reflecting growth in overall DSM spending by these utilities.

While there is some disparity in the proportion of DSM spending devoted to evaluation among utilities that have active evaluation programs, with the exception of 1 utility in the first year of its evaluation activities, none of the 20 participating utilities had devoted more than 7 percent of DSM spending to evaluation activity in any year.

\section{Figure FE4. DSM Evaluation Spending for Selected U.S. Electric Utilities, 1990 through 1994}

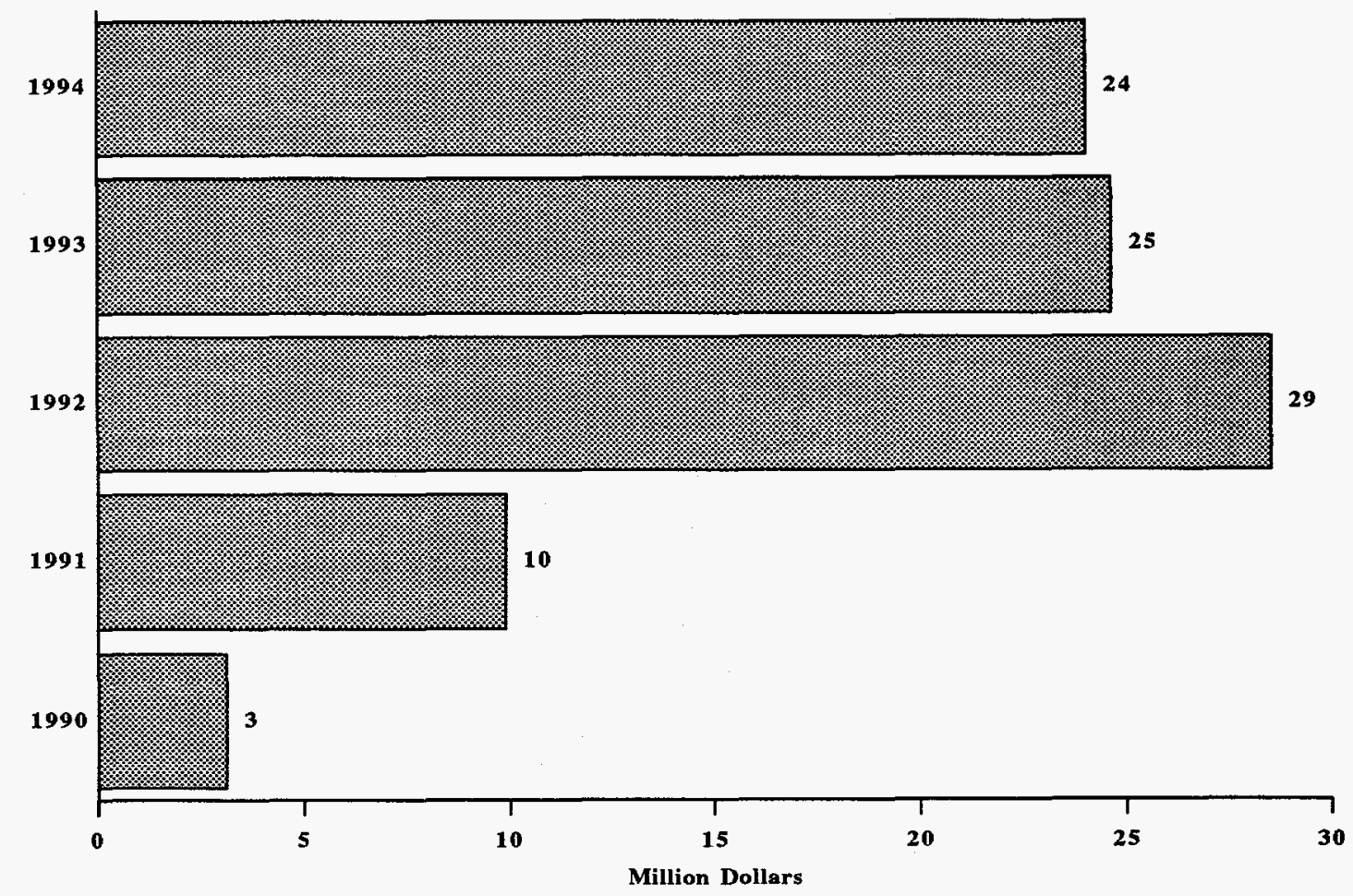

Source: Office of Coal, Nuclear, Electric and Alternate Fuels, DSM Verification Study of 25 Selected U.S. Electric Utilities. 
Figure FE5. Average DSM Evaluation Spending and DSM Evaluation Spending as a Percentage of Total DSM Spending for Selected U.S. Electric Utilities, 1990 through 1994

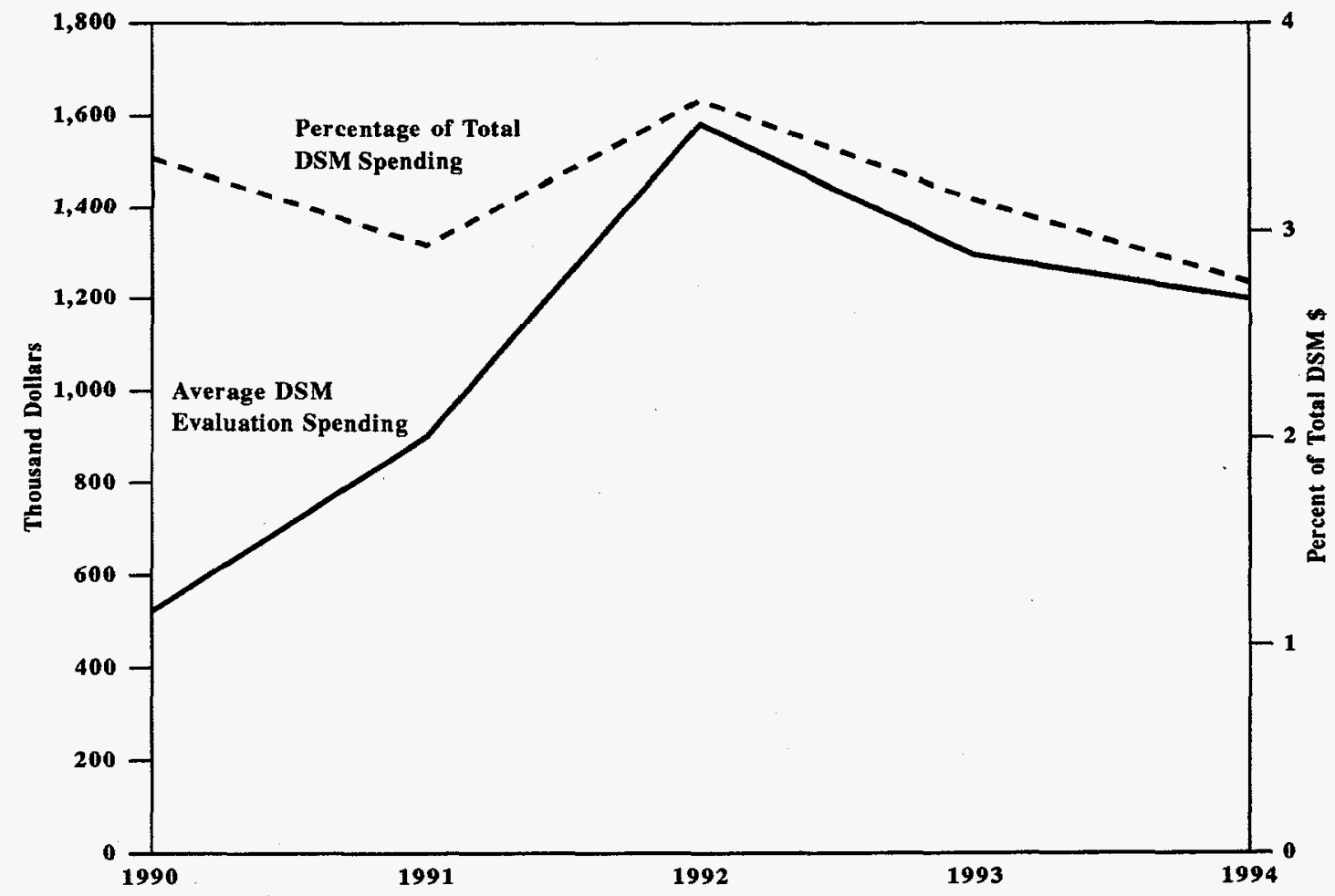

Sources: Office of Coal, Nuclear, Electric and Alternate Fuels, DSM Verification Study of 25 Selected U.S. Electric Utllities, and Energy Information Administration, Form EIA-861

"Annual Electric Utility Report."

\section{Use of Prior Year Evaluation Results}

With the exception of some interruptible load, standby generation, and direct load control programs for which the results of activating peakload reductions may be immediately measurable by the utility, it typically takes some period of time after the completion of a program year before an impact evaluation can be completed. Indeed, during the completion of this study in early 1995, there were a number of DSM programs for which 1993 evaluations were not yet complete. The utilities in the study were asked to give us the typical length of time after the completion of a program year when evaluation results might become available. Eighteen utilities responded to this question. The time frame for evaluation studies ranged from 2 months to 2 years. The average response for the 18 utilities that provided typical lengths of time for evaluation results to be completed was 13.4 months.

Because the due date for utilities to file the Form EIA-861 is May 1 of the year following the reporting year, the results of impact evaluations for many DSM programs for the reporting year will not be available at the time the Form EIA-861 is completed. However, in many instances, the results of prior year evaluations may be available and may provide a basis for estimating savings in the year for which data are reported. The utilities were asked whether they incorporated the results of prior year evaluations in estimating DSM impacts. Of the 25 utilities that answered this question, 16 indicated that they did incorporate results of prior year evaluations, 4 indicated that they did not, and 5 had not completed any evaluations.

\section{Portion of Peakload Reductions and Energy Savings that are Subject to Evaluation Studies}

One of the objectives of this study was to determine the extent to which the DSM activities reported to EIA were subject to some form of utility program evaluation or verification activity. To assess this, the 25 utilities that participated in the study were asked what percentage of total energy savings and of peakload reductions for the 1993 program year are associated with DSM programs for which the utility has conducted an impact evaluation. For those utilities with peakload reduction programs, 19 responded that they had either conducted no evaluations with respect to peakload programs (i.e., 0 percent), or they provided the specific percentage of peakload reduction programs that undergo evaluations. For those utilities with energy savings programs, 17 responded that they had either conducted no evaluations for energy 
savings programs (i.e., 0 percent), or they provided the specific percentage of programs that underwent evaluations. Some of the utilities had conducted impact evaluations for virtually all of their DSM programs. Ten utilities said that 85 percent of their energy savings were from programs for which the utility had conducted impact evaluations, and similarly, ten utilities also stated that 85 percent of their peakload reductions were from programs for which impact evaluations had been performed. The total percentage of peakload reductions and energy savings resulting from programs for which these utilities had conducted impact evaluations, including those with 0 percent, was calculated. The total percentage of peakload reduction programs for which impact evaluations had been performed was 68.1 percent, and the total percentage of energy savings for which impact evaluations had been performed was 73.8 percent.

\section{Evaluation Methodology Utilized}

To provide a rough indicator of the quality of the underlying evaluations, the 25 utilities in the study were asked to indicate the methodologies that had been used in their evaluations of major energy saving and peakload reduction programs for 1993. The results indicate utilization of a broad distribution of accepted methodologies. For 20 utilities that had completed impact evaluations, the methods and number of utilities employing each method are listed below:

Site Visits and Verification of Installation, 16

Measurement of Hours of Usage, 13

Statistically Adjusted Engineering Models, 13

Site-Specific Engineering Analysis, 12

Survey Analysis of Free Ridership, 11

Statistical Analysis of Billing Data, 10

End-Use Metering, 10

Whole Building Metering and Analysis of Specific Energy Uses, 9

\section{Stipulated Savings Based on Estimation} Protocol or Agreement, 1

Only 1 of the 20 utilities did not rely on some form of statistical billing analysis or a direct metering methodology. This suggests that virtually all of the participating utilities that have conducted impact evaluations have some experience with experimental evaluation design.

\section{Realization Rates}

The results of impact evaluations are often presented in terms of the "realization rate" or the percentage of expected energy or peakload savings verified through the evaluation study. ${ }^{27}$ The utilities in the study were asked to provide information comparing the 1993 DSM savings from their programs reported on Form EIA-861 with the results of impact evaluations for the same programs.

Based on the information provided by utilities, realization rates were calculated for peakload reductions for 25 major programs. Similar realization rate calculations were made with respect to energy savings for 24 major programs. Calculated on a weighted average basis (weighted by Form EIA-861 reported savings), the average peakload reduction realization rate for these programs was 88.3 percent. There was variation between the high and low reported realization rate for individual programs, which ranged from 48 percent to 146 percent. Two-thirds of the reported realization rates, however, fell within a narrower band from 75 percent to 115 percent. For energy savings, the weighted average realization rate was 84.5 percent. For energy savings, there was a larger spread between the lowest achieved realization rate of 39 percent and the highest realization rate of 172 percent. However, here again, two-thirds of the individual program realization rates fell within a narrower band between 75 percent and 115 percent.

The average realization rates for the utilities in the study, though below 100 percent, are reasonably high. It is important to remember that EIA records savings only by general program type (energy efficiency, direct load control, interruptible load, other load management, and other DSM) and major customer class. Thus, variations in the realization rates of individual programs could tend to balance out in EIA- reported data as low individual program realization rates could be offset by higher ones.

\section{State Regulatory Requirements}

The investor-owned utilities that participated in the study were located in 19 different States. Of those States, 14 had some specific requirement for the development of evaluation plans or performance of impact evaluations. These requirements ranged from detailed program evaluation protocols ${ }^{28}$ and commission guidelines ${ }^{29}$ to requirements that utilities develop and receive advance approval for evaluation plans that are appropriate to their DSM programs. With the exception of one utility in a State that adopted its evaluation requirements in December 1994, every participating investor-owned utility from a State where

27 Our use of the term "realization rate" here parallels that found in evaluation studies, because savings reported to EIA are often based in part on the installation of DSM measures reported in the utilities' tracking system.

28 California Public Utilities Commission, Protocols and Procedures for the Verification of Costs, Benefits, and Shareholder Earnings from DemandSide Management Programs (Revised July 21, 1994). (1990).

29 New York Public Service Commission, Guidelines in Uniform Format for the Filing of Demand-Side Management Program Evaluation Plans 
the commission had some form of evaluation requirement had completed or was undertaking DSM program impact evaluations. By contrast, only two of the investor-owned utilities from the States lacking evaluation requirements had completed any impact evaluations during the years 1990 through 1994 or had any evaluations underway. These evaluation requirements appear to have had a substantial impact on the development of program evaluation practices by the participating utilities.

Additionally, each of the three noninvestor-owned utilities that participated in this study had undertaken impact evaluations of their programs. Two of these utilities had completed evaluations for all of their DSM programs. The other utility had completed evaluations for programs that represented two-thirds of its energy savings.

\section{Conclusions and Recommendations}

The data reported on Form EIA-861, together with the results of the study, support the observation that there has been a substantial increase in DSM impact evaluation activities since 1990, that large numbers of programs have been evaluated, and that these programs account for a substantial majority of the savings reported to EIA by the utilities that participated in the study. While care must be used in generalizing from the study of a small sample of individual programs, the realization rates calculated herein suggest that evaluation results indicate the aggregate peakload reductions and energy savings reported on the Form EIA-861 are reasonable estimates. Moreover, with increasing State commission requirements for and review of impact evaluations, the growth of evaluations during the 1990's, and the use of prior year evaluations in estimating energy savings by most of the participating utilities, one might expect improvements in the relationship of estimated savings reported on Form EIA-861 to evaluation results in years after 1993. 


\section{Profile: U.S. Electric Utility Demand-Side Management}

This chapter provides a background of electric utility demand-side management in the United States and summarizes pertinent statistics for large utilities ${ }^{1}$ in the United States on various aspects of demand-side management for the year.

\section{Background}

Demand-Side Management (DSM) consists of electric utilities' planning, implementing, and monitoring of activities designed to encourage consumers to modify their level and pattern of electricity consumption to mutually benefit the utility, consumer, and society. Utilities implement DSM programs to achieve two basic objectives: energy efficiency and load management. Energy efficiency is primarily achieved through programs that focus on reducing overall energy consumption of specific end-use devices and systems through the promotion of high-efficiency equipment and building design, typically reducing energy consumption throughout many hours of the year. Load management programs are designed to achieve peakload reduction and are primarily activated at the time of a utility's peak load. Load management programs have little effect on total energy consumption. Electric utilities have steadily increased DSM programs in the last decade to promote energy efficiency, reduce toxic air emissions, and achieve cost effectiveness for both the utility and the consumer, mainly by deferring the need to build new power plants.

The Energy Information Administration (EIA) collects data on DSM programs using six program categories:

Energy Efficiency programs are aimed at reducing the energy used by specific end-use devices and systems, without reducing the quality of energy services provided. These programs reduce overall electricity consumption over many hours during the year, although the greatest impacts of cost-effective programs often coincide with periods of peak usage. Such savings are generally achieved by substituting technically more advanced equipment to produce an equal level of energy services (e.g. lighting, heating, motor drive) with less electricity. Examples include high-efficiency appliances, efficient lighting, high-efficiency heating, ventilating and air conditioning (HVAC) systems or control modification, efficient building design, advanced electric motors and drive systems, and heat recovery systems. Energy efficiency programs frequently incorporate rebates, financing, or other financial incentives for participation.

Direct Load Control represents the consumer load that can be interrupted during the periods of peak load by direct control of the utility system operator. Direct Load Control usually involves residential consumers who, for example, experience periodic interruptions in service to air conditioning by the utility during the hours of peak load.

Interruptible Load accounts for the consumer load that, in accordance with contractual arrangements, can be interrupted during periods of peak load either by the direct control of the utility system operator or by the action of the consumer at the direct request of the system operator. For example, large commercial and industrial consumers may obtain interruptible rates which provide a discount in return for their contractual agreement to reduce electrical loads upon request from the utility, usually as a strategy to reduce peak load.

Other Load Management refers to programs other than direct load control and interruptible load that limit peak loads, or shift peak load from on-peak to off-peak time periods, or encourage customers to respond to changes in the utility's cost of providing power. ${ }^{2}$ It includes technologies that primarily shift all or part of a load from one time of day to another and secondarily may have an impact on energy consumption. Examples include space heating and water heating storage systems, cool storage systems, and load limiting devices in energy management systems. This category also includes programs that aggressively promote time-of-use (TOU) rates and other innovative rates such as real-time pricing. These rates are intended to reduce consumer bills and shift hours of operation of equipment from on-peak to off-peak or

1 Large utilities are those with sales to ultimate consumers or sales for resale greater or equal to $120,000 \mathrm{MWh}$ annually. These utilities are required to complete the entire Schedule V, "Demand- Side Management Information," of the Form EIA-861, "Annual Electric Utility Report." Small utilities with sales to ultimate consumers and sales for resale less than $120,000 \mathrm{MWh}$ annually are required to report only incremental effects, total utility costs, total nonutility costs, and total DSM costs for the reporting year and the first and fifth forecast years. The data presented in this publication are for only large utilities, unless otherwise stated.

2 Load control mechanisms such as interruptible load programs may be used in emergency situations. However, sometimes other load control mechanisms such as voltage reduction or rolling blackouts may be needed. While voltage reduction and rolling blackouts reduce load and save energy, they are not considered DSM programs. Voltage Reduction is further described in the Technical Notes. 
high-cost to low-cost periods through the application of time-differentiated rates.

Other Demand-Side Management are those programs that capture effects of DSM programs that cannot be meaningfully included in any of the other program categories. Programs that promote consumer's substitution of other energy types for electricity and selfgeneration of electricity for the consumer's own use are included.

Load Building programs are aimed at increasing the use of existing electric equipment or the addition of electric equipment. Examples include industrial technologies such as induction heating and melting, direct arc furnaces and infrared drying; cooking for commercial establishments; and heat pumps for residences. Load Building includes programs that promote the substitution of electricity for other fuels. ${ }^{3}$

The concept of energy efficiency began in the 1970's in response to increasing capital costs, increasing consumer demand for electricity, rising electricity prices, and public awareness of limited energy resources. Federal regulators and State utility commissions responded with utility policies that contributed to the evolution of DSM. Federal legislation includes the Energy Policy and Conservation Act (1975), Energy Conservation and Production Act (1976), and National Energy Conservation Policy Act (1978). These three Acts provided the technical basis for utility conservation and load management programs. The Public Utility Regulatory Policies Act (1978) encouraged efficiency and more economically efficient electric rates by requiring State commissions to consider rate-making standards that further the purposes of end-use conservation, utility efficiency, and equitable rates. It required State commissions to review cost allocations across consumer classes, the accuracy of declining block rates in reflecting actual costs, time-of-day and seasonal rates, interruptible rates, and load management techniques. The Pacific Northwest Electric Power Planning and Conservation Act (1980) and Hoover Power Plant Act (1984) encouraged DSM through Federal power marketing authorities.

The National Appliance Energy Conservation Act (1987), Clean Air Act and its Amendments (1990), and the Energy Policy Act (1992) are the most recent Federal legislation affecting DSM. The Clean Air Act Amendments of 1990 are important to DSM because they internalized the cost of environmental externalities, specifically sulfur dioxide emissions, through the adoption of a market-based system of emission control in which utilities are issued allowances, each allowing the emission of one ton of sulfur dioxide per year. This system encourages utilities to reduce emissions in the most cost effective manner and sell excess allowances for a profit.

The Energy Policy Act of 1992 (EPACT) represents the continuing Federal interest in encouraging energy efficiency. EPACT requires State utility commissions to consider standards that will require utilities to employ Integrated Resource Planning (IRP). Therefore, the most significant regulatory requirements affecting DSM are occurring at the State level. The IRP process also had its beginning in the 1970's when many States began to review utilities' plans for forecasting and siting of new generation capacity in response to the completion of controversial capacity additions over which there had been limited or no advance regulatory oversight. A majority of States now require IRP, and a continued interest in DSM can be partially attributed to it. IRP differs from conventional resource planning in that utilities consider both demand- and supply-side resources as options for meeting future electricity requirements, rather than consider only supply-side resources. Specifically, a utility is able to assume a decrease in demand as a result of DSM programs when planning to meet future electricity demands, rather than increasing generation methods.

DSM programs are a key component of IRP at a growing number of utilities. IRP differs among utilities based on availability of resources, owners of the resources, organizations involved in planning, and the criteria for resource selection. The IRP process is complex, taking into consideration the balance of supply- and demand-side resources, risk and diversity of supply, system reliability maintenance, external changes (economic conditions), energy prices, new technologies, regulatory and tax policy changes, and in some instances, the application of specific values to reflect environmental and other externalities.

The key elements of the DSM program planning and selection process are to identify and evaluate consumer characteristics that influence acceptance of and response to DSM programs and to identify utility considerations that affect resource requirements and the cost of alternative resource options. Among consumer characteristics that influence the success of a program are demographics, income, knowledge, awareness, attitude, and motivation. External influences such as economic conditions, energy prices, technologies, regulation, and tax credits also influence consumer's decisions regarding fuel and appliance choices, appliance and equipment efficiency, and appliance use. The utility's considerations are usually focused on the interaction of load shape distribution effects and regulatory compliance.

Due to the regulated nature of the electric power industry, utilities maximize revenue by selling as much electricity as possible; thus, DSM programs that reduce sales should not interest them. To promote DSM, State regulatory commissions developed financial incentives, such as authorizing utilities to seek recovery of DSM program costs and lost revenues and granting the utility a higher rate of return. These incentives are meant to neutralize the lost sales and revenues attributable to DSM. To compare DSM programs to other demand- and supply-side resources, 
regulators have developed standardized benefit/cost tests. Four primary tests are widely used in planning to identify cost-effective DSM programs. For each test, the net present value and benefit/cost ratio can be determined. The present value equals total benefits of the program less total cost; the benefit/cost ratio is the ratio of total benefits to total costs. Based on these values, the utility can prioritize DSM programs to determine which, if any, might be implemented.

The Utility Cost Test measures the net change in a utility's revenue requirement resulting from a DSM program. The test compares the reduction in marginal energy and demand costs with utility program costs, incentive payments, and increased supply costs for a period in which load is increased. Designed to focus on a utility's revenue requirement, the test does not include any net costs incurred by participants.

The Participant Cost Test measures the benefits and costs of a DSM program to a customer by comparing the reduction in the customer's utility bill, plus any incentive paid by the utility, with the customer's outof-pocket expenses. The test is often used as a "first-cut" in ranking program desirability and gauging potential program participation rates.

The Total Resource Cost Test measures the net costs of a DSM program as a resource option based on the total costs of the program, including both participant and utility costs. Like the utility cost test, it measures benefits as reductions to energy and demand costs, but also includes a review of all program costs, including installation, operation, maintenance, and administration, no matter who pays for them.

The Rate Impact Measure Test measures the direction and magnitude of the expected changes in rates for all customers when a utility implements a DSM program. The equation functions initially in the same manner as the utility cost test, comparing avoided supply cost savings with cost to the utility. It also, however, measures the revenue-shifting effect unique to DSM --that is, when costs must be spread over a smaller sales volume. The shift reduces revenue requirements, but not to the same extent as sales are reduced by DSM programs. The difference causes an increase in rates on a cents per kilowatthcur basis. If a utility has excess capacity and its average costs exceeds its marginal costs, a DSM program will likely increase rates. The converse is true when marginal costs are forecast to exceed average costs.

\section{Current Issues and Trends}

Recent changes in the electric utility industry will likely have major impacts on the future of DSM. The possibility of competition in the industry has led to the speculation that DSM could decline. According to a study reported in Public Utility Fortnightly, in 1994, 67 percent of State regulators believed that there would be a decline in electric utility DSM as a result of utility deregulation. This is a 100-percent increase over the 33 percent of State regulators who believed in 1993 that there would be a decline. Regulators believe that a market will develop with private companies providing DSM services, as opposed to utility sponsored programs. The California Public Utilities Commission stated that DSM will not decline but "a competitive market will guide consumers to efficient energy use." 4

The greatest response to the changes in the electric power industry have been observed in California. Pacific Gas and Electric proposed to decrease their DSM expenditures in an attempt to cover an electric rate freeze, ${ }^{5}$ and Southern California Edison plans to cut 1995 DSM expenditures as a part of their 1995 general rate case. In Michigan, Consumers Power unsuccessfully petitioned the Michigan Public Service Commission to reduce by half the utility's DSM expenditures because of increased competition. ${ }^{6}$

In 1993, 977 of the 3,212 electric utilities in the United States reported having DSM programs, an increase of 8 percent over 1992. Of these, 149 were investorowned utilities, 413 were cooperatives, 413 were publicly owned, and 2 were Federally owned (Figure 1). These 977 utilities accounted for 86 percent of total retail sales of electricity in the United States. (Figure 1). Also, of the 977,545 are classified as large utilities and 432 are small utilities. The 545 large utilities account for 84 percent of the total retail sales of electricity in the United States. The number of large utilities increased by 8 percent from 1992 when 504 reported having DSM programs and small utilities increased by 8 percent, from 401, in 1992 .

In 1993, energy savings for the 545 large utilities increased to 45,294 million $\mathrm{kWh}, 9,731$ million $\mathrm{kWh}$ over the 35,563 million $\mathrm{kWh}$ reported in 1992. These energy savings represented 1.6 percent of the total annual electric sales in 1993 of $2,861,462$ million $\mathrm{kWh}^{7}$

4 R. Rudden and J. Rosenbloom, "Competitive Forces and Market Risks: Regulators' Views on the Future Electric Utility Industry," Public Utility Fortnightly, (January 1, 1995) p. 9.

5 "PG\&E Confirms Plans to Slash 1995 DSM Budget by \$100-Million; Rate Freeze Cited," Demand-Side Report (September 1, 1994) p.1.

6 "Michigan Says Consumers' Competitive Fears Are 'Vague'; Nixes DSM Budget Cut," Demand-Side Report (September 15, 1994$)$ p.1.

7 Energy Information Administration, Electric Sales and Revenue 1993, DOE/EIA0540(93) (Washington, DC, January 1995) Table 1, p. 5. 
Since 1989, annual energy savings increased at an average annual rate of 33 percent. Actual peakload reductions in 1993 were $23,069 \mathrm{MW}$, an increase of 34 percent over 1992. These actual peakload reductions are estimated to be approximately 3 percent of total peak load in the United States. Potential peakload reductions in 1993 were $39,508 \mathrm{MW}$, an increase of 22 percent over 1992. DSM cost was approximately $\$ 2.7$ billion, an increase of 17 percent over 1992, and an average annual increase of 33 percent or $\$ 1.9$ billion since 1989 (Table 1).

Incremental effects are those caused by new programs and new participants in existing programs for the current reporting year. For 1993, incremental energy savings were 8,980 million $\mathrm{kWh}$, incremental actual peakload reductions were 4,648 MW, and incremental potential peakload reductions were 7,586 MW (Figure 2). ${ }^{8}$

\section{Table 1. U.S. Electric Utility DSM Program Energy Savings, Actual and Potential Peakload Reductions, and Cost, 1989 Through 1993}

\begin{tabular}{|c|c|c|c|c|c|}
\hline Item & 1989 & 1990 & 1991 & 1992 & 1993 \\
\hline 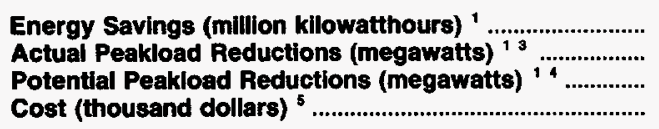 & $\begin{array}{r}14,672 \\
12,463 \\
N A \\
872,935\end{array}$ & $\begin{array}{r}20,458 \\
213,704 \\
\text { NA } \\
1,177,457\end{array}$ & $\begin{array}{r}224,848 \\
215,619 \\
N A \\
21,803,773\end{array}$ & $\begin{array}{r}35,563 \\
17,204 \\
32,442 \\
22,348,094\end{array}$ & $\begin{array}{r}45,294 \\
23,069 \\
39,508 \\
2,743,533\end{array}$ \\
\hline
\end{tabular}

Represents the total annual effects caused by all participants in demand-side management programs in effect during a given year. Included are new and existing participants in existing programs (those implemented in prior years that are in place during the reporting year) and all participants in new programs (those implemented during the reporting year).

2 Due to data corrections by several large utilities, totals may not equal those previously published in the Electric Power Annual 1993. (See Technical Notes.)

3 Represents the actual reduction in annual peak load achieved by consumers in the following demand-side management program categories: energy efficiency, direct load control, interruptible load, other load management, other demand-side management; reflects real changes in the demand for electricity at the time of annual peak load, as opposed to the installed peakload reduction capability (i.e., Potential Peakload Reduction).

- Represents the potential reduction in annual peak load installed in the following demand-side management program categories: direct load control, interruptible load, other load management, other demand-side management including the actual amount of peakload reductions achieved with

energy efficiency progams; reflects potential changes in the demand for electricity at the time of annual peak load, as opposed to the actual peakload reduction achieved (i.e., Actual Peakload Reduction).

5 Referred to in the Electric Power Annual, 1992 as "Expenditures," these data represent the sum of the direct and indirect utility costs for the year and reflect the total cash expenditures incurred for the year, reported in nominal dollars, that flowed out to support demand-side management programs.

NA = Data not available.

Notes: -Data are final. -Data are provided for electric utilities with sales to ultimate consumers or sales for resale greater than or equal to 120,000 megawatthours. - Totals may not equal sum of components because of independent rounding.

Source: Energy Information Administration, Form EIA-861, "Annual Electric Utility Report."

8 It is incorrect to assume that 1992 annual effects plus 1993 incremental effects are equal to 1993 annual effects. Reasons for this include incremental effects being annualized, and participants dropping out of programs that are not included in incremental effects. 
Figure 1. Number of U.S. Electric Utilities With and Without DSM Programs, 1993

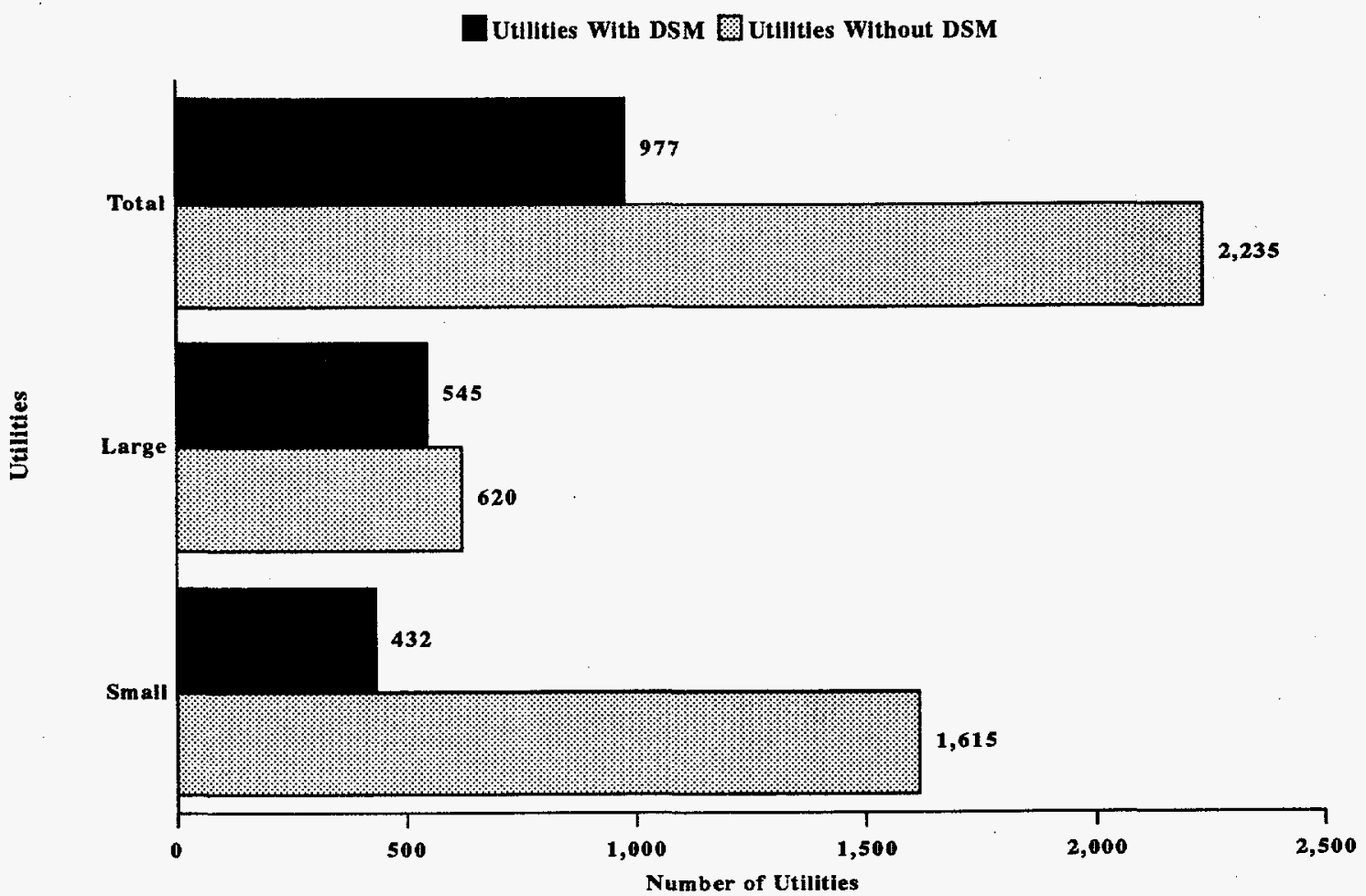

Source: Energy Information Administration, Form EIA-861, "Annual Electric Utility Report."

Figure 2. U.S. Electric Utility DSM Program Incremental and Annual Effects for Energy Savings and Actual and Potential Peakload Reductions, 1993

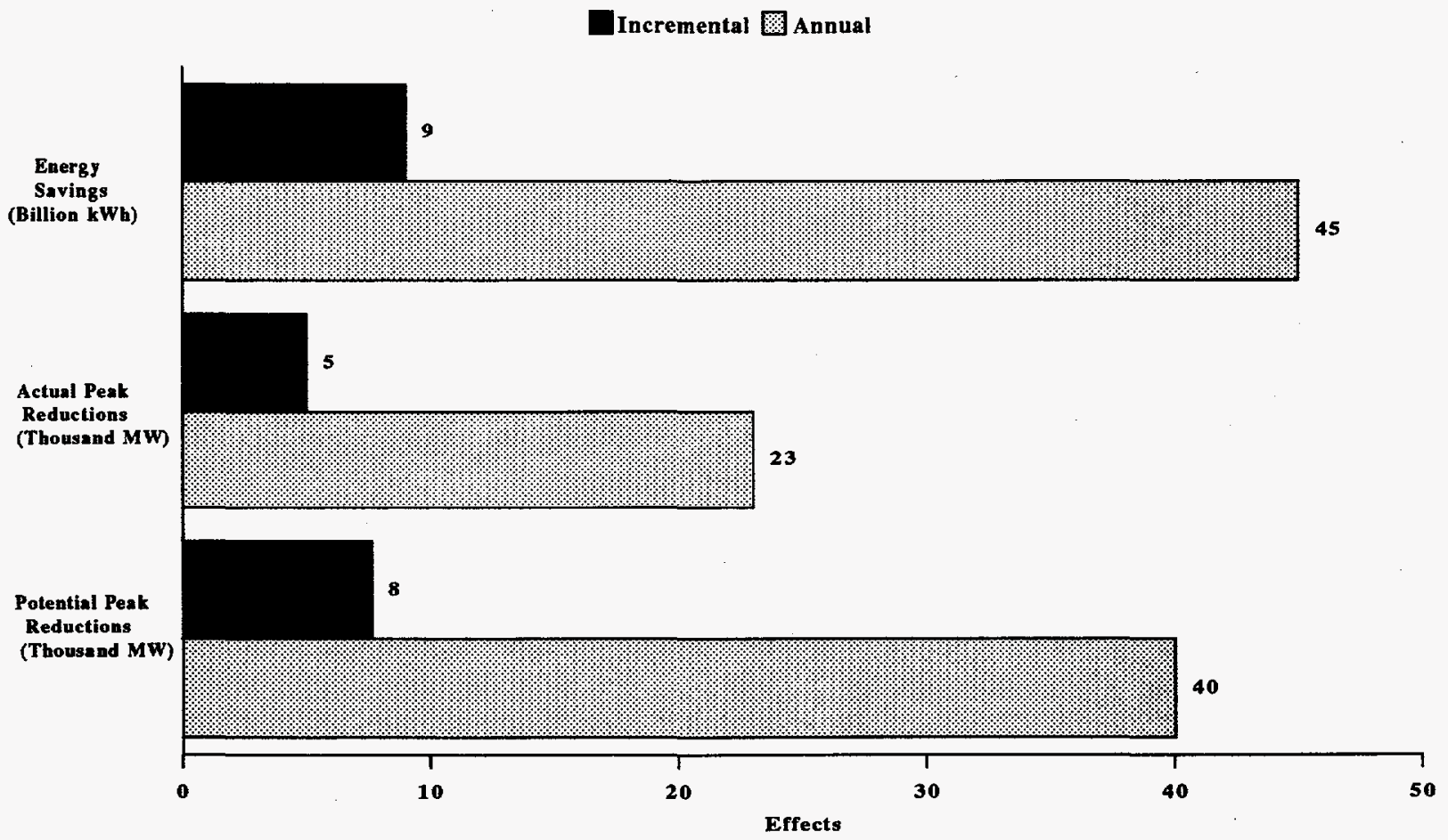

Source: Energy Information Administration, Form EIA-861, "Annual Electric Utility Report." 



\section{Energy Savings}

Energy savings represent a decrease in the amount of electricity (measured in kilowatthours $(\mathrm{kWh})$ ) used. Energy savings primarily result from energy efficiency programs, but also result from load management and other DSM programs. Examples of energy efficiency programs include the use of highly efficient appliances; lighting; heating, ventilating and air conditioning (HVAC) systems or control modification; building design; advanced electric motors and drive systems; and heat recovery systems.

In 1993, energy savings increased 27 percent to 45,294 million $\mathrm{kWh}$, from the 1992 level of 35,563 million $\mathrm{kWh}$, although the rate of growth in energy savings for 1993 declined slightly from the average annual rate of 30 percent from 1989 to 1992. In 1994, energy savings were forecast to increase 16 percent to 52,655 million $\mathrm{kWh}$, and by 1998 , to increase at an average annual rate of 14 percent to 88,978 million $\mathrm{kWh}$ (Table 2).

The year's energy savings represented a reduction in electricity sales by electric utilities of 1.6 percent. ${ }^{9}$ Less than 15 percent of utilities with DSM programs reduced their energy sales by more than 1 percent in 1993 (Figure 3). Investor-owned utilities represented the greatest energy savings as a percentage of sales in 1993.

The 100 utilities with the greatest energy savings accounted for 96 percent of the total energy savings. The 50 utilities with the greatest energy savings accounted for 87 percent of the total and the 25 top utilities accounted for 75 percent of energy savings (Figure 4). These 100, 50, and 25 utilities with the greatest energy savings represented 58 percent, 37 percent, and 26 percent, respectively, of the total retail sales of electricity in the United States for 1993.

Investor-owned utilities accounted for 77 percent of energy savings in 1993, Federally owned utilities accounted for 15 percent, publicly owned, 6 percent, and cooperatives, 2 percent. ${ }^{10}$ However, cooperatives increased energy savings 76 percent over their 1992 level. The greatest energy savings were achieved at investor-owned utilities with 9,151 million $\mathrm{kWh}$ more in 1993 than in 1992. For 1994, Federally owned utilities predicted the greatest percentage of increase, 26 percent more than $1993,1,788$ million $\mathrm{kWh}$; and investor-owned utilities predicted an increase of 4,945 million $\mathrm{kWh}$. Cooperatives predicted the greatest average annual rate of increase, 26 percent, from 1994 to 1998 . Investor-owned utilities predicted the greatest $\mathrm{kWh}$ increase for 1998, 23,814 million $\mathrm{kWh}$ (Table 2).

In 1993, energy efficiency programs accounted for 91 percent of the energy savings because the primary objective of this type of programs is energy savings while the primary objective of most other DSM programs is peakload reduction. Direct load control, interruptible load, other load management, and other DSM programs together accounted for the remaining 9 percent of energy savings. Energy savings as a result of energy efficiency programs increased 29 percent since 1992. The greatest percentage of increase, 40 percent, from 1992 to 1993 was in other DSM programs. For 1994, energy efficiency programs are predicted to continue to account for the greatest percent of energy savings, 93 percent. The greatest percentage of increase is predicted for direct load control programs, expected to increase 47 percent by 1994. By 1998 , energy efficiency programs are expected to increase energy savings by an additional 30,812 million kWh over projected 1994 levels (Table 3).

During the year, more utilities reported having energy efficiency programs in place in the residential sector than in the commercial or industrial sectors. However, the commercial and industrial sectors still contributed a large percentage of energy savings due to economies of scale (i.e., a commercial building participating in an efficient lighting program will have greater energy savings than a single residential building). Energy efficiency end-use and program types in the residential sector were primarily for heating systems, cooling systems, and water heating. More utilities had lighting and cooling systems for the commercial sector, and in the industrial sector, the focus was on lighting and motors. Across all sectors, more utilities used energy audits than other programs, followed by rebates, loans, other incentives, and other programs (Table 4).

The residential sector accounted for 42 percent of energy savings in 1993, followed by the commercial, industrial, and other sectors with 37 percent, 19 percent, and 2 percent, respectively. The greatest percentage of increase from 1992 to 1993 was in the commercial sector, with an increase of 35 percent, mainly due to an increase in utility-administered efficient lighting programs (Table 5).

In 1993, incremental energy savings (the savings achieved by new programs and new participants in existing programs in a given year) increased from

9 Total U.S. electric utility sales for 1993 were $2,861,462$ million $\mathbf{k W h}$.

10 Investor-owned utilities accounted for 76 percent of total sales, publicly owned utilities, 14 percent, cooperatives, 8 percent, and Federally owned utilities, 2 percent. 
6,664 million $\mathrm{kWh}$ in 1992 to 8,980 million $\mathrm{kWh}$ for large utilities and decreased from 48 million $\mathrm{kWh}$ to 22 million kWh for small utilities. By class of ownership, large investor-owned utilities accounted for the greatest percentage of incremental energy savings at 85 percent, and the greatest incremental increase in $\mathrm{kWh}$, from 5,642 million $\mathrm{kWh}$ in 1992 to 7,639 million $\mathrm{kWh}$ in 1993 (Table 6).

By program category, the greatest increase during the year in incremental energy savings was achieved by large utilities with energy efficiency programs, which increased 2,258 million $\mathrm{kWh}$ over the 1992 level of 6,214 million $\mathrm{kWh}$. The greatest percentage of increase from 1992 to 1993 was in the other DSM category, which increased 124 percent. For all remaining program categories, large utilities decreased in incremental savings. For small utilities in 1993, other DSM programs had incremental savings that remained the same as 1992 incremental levels, and all other categories of programs decreased (Table 7).

The commercial sector accounted for the greatest incremental savings, 4,557 million $\mathrm{kWh}$, in 1993 for large utilities, amounting to 51 percent of the total incremental savings. The commercial sector also accounted for the greatest increase over the 1992 level of incremental energy savings, increasing by 1,224 million $\mathrm{kWh}$. The residential sector contributed 2,780 million $\mathrm{kWh}, 31$ percent, of total incremental energy savings. The greatest percentage of increase over 1992 was achieved by the industrial sector which increased by 50 percent (Table 8 ).

The NERC region with the greatest percentage of energy savings was Western Systems Coordinating Council (WSCC), accounting for 40 percent of energy savings in 1993. The primary reason the WSCC had the most energy savings is that Bonneville Power Administration and Southern California Edison had the two largest energy efficiency programs of all electric utilities. The region with the second largest energy savings was Southeastern Electric Reliability Council (SERC), with 25 percent of total energy savings. In 1992, these two regions combined accounted for 70 percent of total energy savings in the United States. WSCC and Northeast Power Coordinating Council (NPCC) both reduced energy sales by 3 percent as a result of their DSM programs. The remaining regions had reductions in energy sales of less than 1.5 percent attributable to DSM programs (Tables 9 and 10).

For 1994, the greatest percentage of increase, 91 percent, in energy savings is predicted for the MidAtlantic Area Council (MAAC) region, primarily due to large increases by Public Service Electric and Gas and Potomac Electric and Power Company. The greatest increase in $\mathrm{kWh}$ in both 1994 and 1998 is expected for the WSCC region. The East Central Area Reliability Coordination Agreement (ECAR) region is expected to have the greatest annual rate of growth between 1994 and 1998 at 26 percent (Table 9).

Table 2. U.S. Electric Utility DSM Program Energy Savings by Class of Ownership, 1989 Through 1993, 1994 and 1998 (Million Kilowatthours)

\begin{tabular}{|c|c|c|c|c|c|c|c|}
\hline \multirow{2}{*}{ Class of Ownership } & \multicolumn{5}{|c|}{ Historical Savings } & \multicolumn{2}{|c|}{ Projected Savings } \\
\hline & 1989 & 1990 & 1991 & 1992 & 1993 & 1994 & 1998 \\
\hline 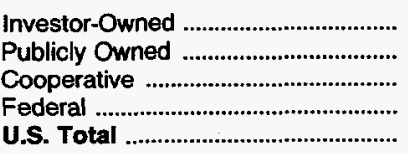 & $\begin{array}{r}8,194 \\
708 \\
158 \\
5,611 \\
14,672\end{array}$ & $\begin{array}{r}13,868 \\
913 \\
94 \\
5,584 \\
20,458\end{array}$ & $\begin{array}{r}17,521 \\
1,448 \\
185 \\
5,695 \\
24,848\end{array}$ & $\begin{array}{r}25,926 \\
2,416 \\
400 \\
6,822 \\
35,563\end{array}$ & $\begin{array}{r}35,077 \\
2,562 \\
705 \\
6,950 \\
45,294\end{array}$ & $\begin{array}{r}40,022 \\
3,062 \\
833 \\
8,738 \\
52,655\end{array}$ & $\begin{array}{r}63,836 \\
5,436 \\
2,036 \\
17,670 \\
88,978\end{array}$ \\
\hline
\end{tabular}

Notes: -Data are final. -Data are provided for electric utilities with sales to ultimate consumers or sales for resale greater than or equal to 120,000 megawatthours.

Source: Energy Information Administration, Form EIA-861, "Annual Electric Utility Report." 
Figure 3. Energy Savings as a Percentage of Retail Sales by U.S. Electric Utilities with DSM Energy Savings Programs and by Class of Ownership, 1993

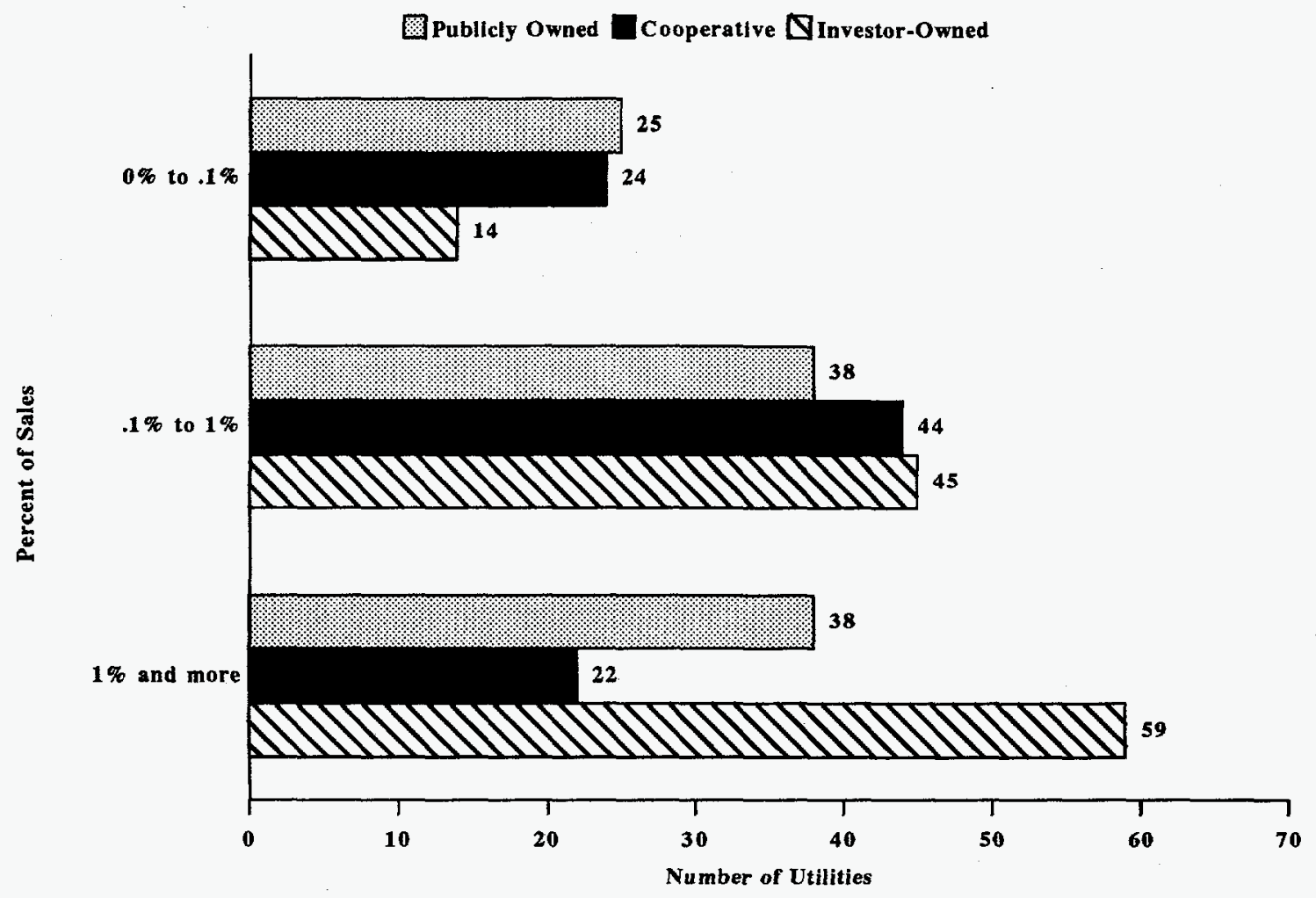

Note: This graph only shows utllities that reported energy savings.

Source: Energy Information Administration, Form EIA-861, "Annual Electric Utility Report." 
Figure 4. The Top 25, 50, and 100 U.S. Electric Utilities with the Greatest DSM Program Energy Savings by Class of Ownership, 1993

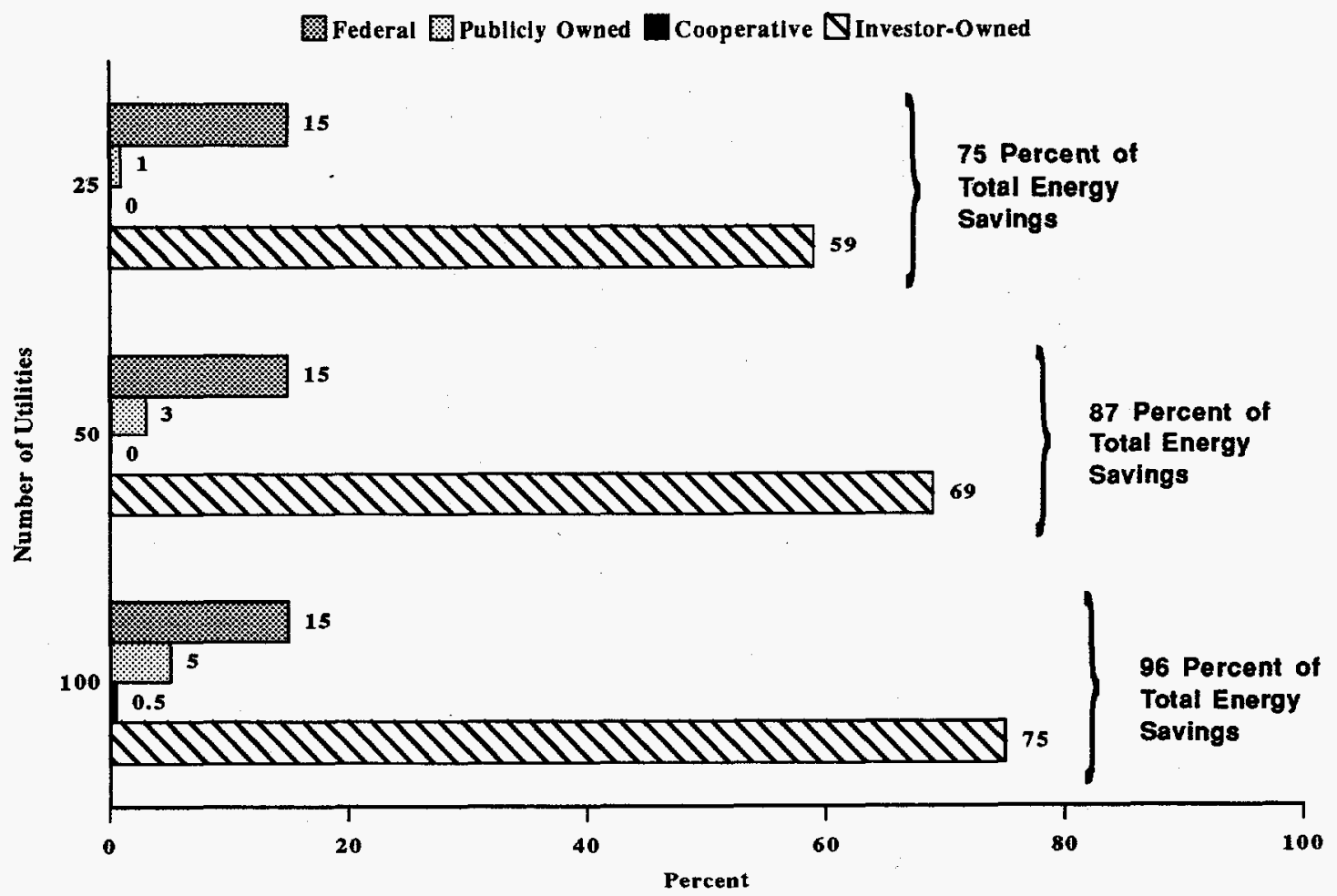

Note: There are no cooperatives in the top 25 or 50 electric utilities.

Source: Energy Information Administration, Form EIA-861, "Annual Electric Utility Report."

Table 3. U.S. Electric Utility DSM Program Energy Savings by Program Category, 1992, 1993, 1994, and 1998

(Million Kilowatthours)

\begin{tabular}{|c|c|c|}
\hline \multirow{2}{*}{ Program Category } & \multicolumn{2}{|c|}{ Historical Savings } \\
\hline & 1992 & 1993 \\
\hline \multirow[t]{3}{*}{ 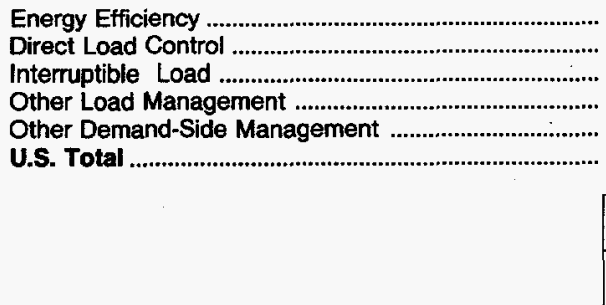 } & $\begin{array}{r}31,779 \\
289 \\
1,994 \\
419 \\
1,081 \\
35,563\end{array}$ & $\begin{array}{r}41,119 \\
319 \\
2,119 \\
223 \\
1,514 \\
45,294\end{array}$ \\
\hline & \multicolumn{2}{|c|}{ Projected Savings } \\
\hline & 1994 & 1998 \\
\hline 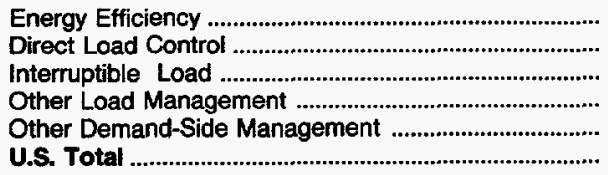 & $\begin{array}{r}48,819 \\
469 \\
1,929 \\
398 \\
1,039 \\
52,655\end{array}$ & $\begin{array}{r}79,631 \\
543 \\
6,178 \\
1,177 \\
1,449 \\
88,978\end{array}$ \\
\hline
\end{tabular}

Notes: -Data are final. $\bullet$ Data are provided for electric utilities with sales to ultimate consumers or sales for resale greater than or equal to 120,000 megawatthours. - Totals may nof equal sum of components because of independent rounding.

Source: Energy Information Administration, Form EIA-861, "Annual Electric Utility Report." 
Table 4. Number of U.S. Electric Utilities with DSM Energy Efficiency Programs by End Uses and Program Types by Sector, 1993

\begin{tabular}{|c|c|c|c|}
\hline \multirow{2}{*}{ ITEM } & \multicolumn{3}{|c|}{ Sectors } \\
\hline & Residential & Commercial & Industrial \\
\hline \multicolumn{4}{|l|}{ End Uses } \\
\hline Heating Sytems & 264 & 172 & 105 \\
\hline 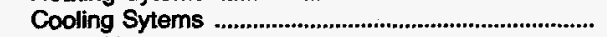 & 271 & 200 & 134 \\
\hline 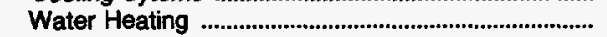 & 283 & 144 & 98 \\
\hline 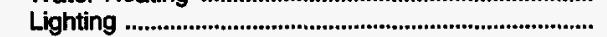 & 182 & 208 & 174 \\
\hline 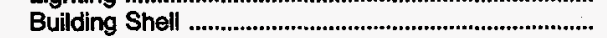 & 186 & 118 & 89 \\
\hline 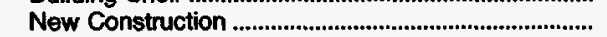 & 195 & 132 & 96 \\
\hline 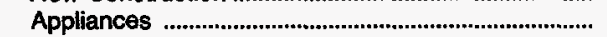 & 131 & 63 & 40 \\
\hline 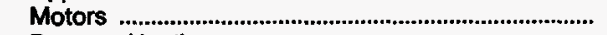 & - & 140 & 151 \\
\hline 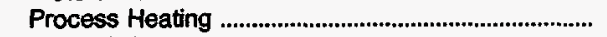 & - & 42 & 81 \\
\hline 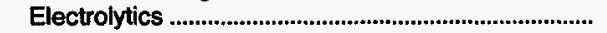 & - & 10 & 26 \\
\hline 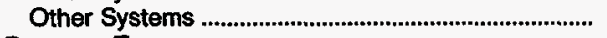 & 20 & 27 & 28 \\
\hline \multicolumn{4}{|l|}{ Program Types } \\
\hline Energy Audits & 290 & 242 & 181 \\
\hline 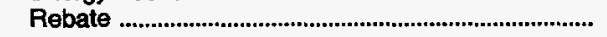 & 271 & 197 & 137 \\
\hline 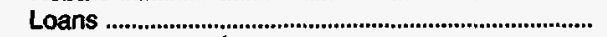 & 139 & 80 & 55 \\
\hline 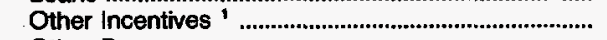 & 92 & 77 & 66 \\
\hline Other Programs & 42 & 37 & 35 \\
\hline
\end{tabular}

1 This category reflects programs that offer cash or noncash awards to electric energy efficiency deliverers, such as appliance and equipment dealers, building contractors, and architectural and engineering firms, that encourage consumer participation in a demand-side management program and adoption of recommended measures. types.

Notes: -Data are final. -Data represent the total number of electric utilities that focus energy efficiency activities on specific end uses and program Source: Energy Information Administration, Form EIA-861, "Annual Electric Utility Report."

Table 5. U.S. Electric Utility DSM Program Energy Savings by Sector, 1992 and 1993 (Million Kilowatthours)

\begin{tabular}{|c|c|c|}
\hline Sector & 1992 & 1993 \\
\hline 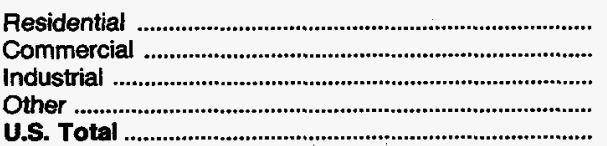 & $\begin{array}{r}15,322 \\
12,301 \\
7,192 \\
748 \\
\mathbf{3 5 , 5 6 3}\end{array}$ & $\begin{array}{r}19,241 \\
16,567 \\
8,644 \\
842 \\
45,294\end{array}$ \\
\hline
\end{tabular}

Notes: -Data are final. Data are provided for electric utilities with sales to ultimate consumers or sales for resale greater than or equal to 120,000 megawatthours. - Totals may not equal sum of components because of independent rounding.

Source: Energy Information Administration, Form ElA-861, "Annual Electric Utility Report." 
Table 6. U.S. Electric Utility Incremental Energy Savings by Class of Ownership, 1992 and 1993 (Million Kilowatthours)

\begin{tabular}{|c|c|c|c|c|c|c|}
\hline \multirow{2}{*}{ Class of Ownership } & \multicolumn{2}{|c|}{ Large Utilties ${ }^{1}$} & \multicolumn{2}{|c|}{ Small Utilities ${ }^{2}$} & \multicolumn{2}{|c|}{ Total } \\
\hline & 1992 & 1993 & 1992 & 1993 & 1992 & 1993 \\
\hline 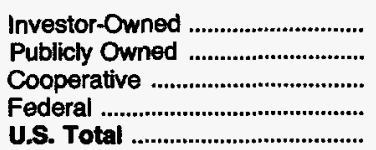 & $\begin{array}{r}5,642 \\
600 \\
66 \\
355 \\
6,664\end{array}$ & $\begin{array}{r}7,639 \\
528 \\
211 \\
601 \\
8,980\end{array}$ & $\begin{array}{r}* \\
17 \\
31 \\
0 \\
48\end{array}$ & $\begin{array}{r}* \\
14 \\
7 \\
0 \\
22\end{array}$ & $\begin{array}{r}5,642 \\
617 \\
98 \\
355 \\
6,712\end{array}$ & $\begin{array}{r}7,639 \\
543 \\
219 \\
601 \\
9,002\end{array}$ \\
\hline
\end{tabular}

1 Refers to electric utilities with sales to ultimate consumers or sales for resale greater than or equal to 120,000 megawatthours.

2 Refers to electric utilities with sales to ultimate consumers and sales for resale less than 120,000 megawatthours.

Notes: -Data are final. -Totals may not equal sum of components because of independent rounding.

Source: Energy Information Administration, Form EIA-861, "Annual Electric Utility Report."

Table 7. U.S. Electric Utility Incremental Energy Savings by Program Category, 1992 and 1993 (Million Kilowatthours)

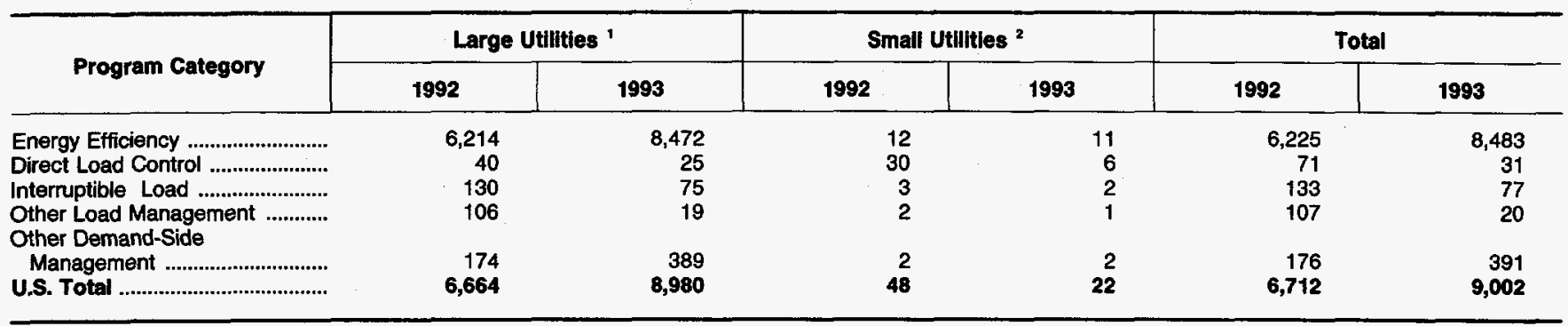

1 Refers to electric utilities with sales to ultimate consumers or sales for resale greater than or equal to 120,000 megawatthours.

2 Refers to electric utilities with sales to ultimate consumers and sales for resale less than 120,000 megawatthours.

Notes: Data are final. - Totals may not equal sum of components because of independent rounding.

Source: Energy Information Administration, Form EIA-861, "Annual Electric Utility Report."

Table 8. U.S. Electric Utility Incremental Energy Savings by Sector, 1992 and 1993 (Million Kilowatthours)

\begin{tabular}{|c|c|c|c|c|c|c|}
\hline \multirow{2}{*}{ Sector } & \multicolumn{2}{|c|}{ Large Utilties ${ }^{9}$} & \multicolumn{2}{|c|}{ Small Utinities ${ }^{2}$} & \multicolumn{2}{|c|}{ Total } \\
\hline & 1992 & 1993 & 1992 & 1993 & 1992 & 1993 \\
\hline 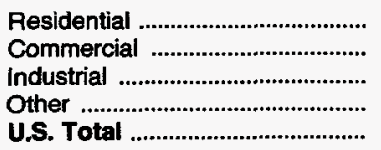 & $\begin{array}{r}2,165 \\
3,333 \\
1,014 \\
151 \\
6,664\end{array}$ & $\begin{array}{r}2,780 \\
4,557 \\
1,518 \\
125 \\
8,980\end{array}$ & $\begin{array}{r}14 \\
5 \\
26 \\
3 \\
48\end{array}$ & $\begin{array}{r}13 \\
4 \\
3 \\
2 \\
22\end{array}$ & $\begin{array}{r}2,179 \\
3,338 \\
1,040 \\
154 \\
6,712\end{array}$ & $\begin{array}{r}2,794 \\
4,561 \\
1,520 \\
127 \\
9,002\end{array}$ \\
\hline
\end{tabular}

1 Refers to electric utilities with sales to ultimate consumers or sales for resale greater than or equal to 120,000 megawatthours.

2 Refers to electric utilities with sales to ultimate consumers and sales for resale less than 120,000 megawatthours.

Notes: -Data are final. -Totals may not equal sum of components because of independent rounding.

Source: Energy Information Administration, Form EIA-861, "Annual Electric Utility Report." 
Table 9. U.S. Electric Utility Energy Savings by North American Electric Reliability Council Region and Hawaii by Class of Ownership, 1992, 1993, 1994 and 1998

(Million Kilowatthours)

\begin{tabular}{|c|c|c|c|c|c|}
\hline \multirow{2}{*}{$\begin{array}{c}\text { North American Electric Reliability } \\
\text { Councll Region and Hawail / } \\
\text { Electric Utility }\end{array}$} & \multirow{2}{*}{$\begin{array}{l}\text { Class of } \\
\text { Ownership }\end{array}$} & \multicolumn{2}{|c|}{ Historical Savings } & \multicolumn{2}{|c|}{ Projected Savings } \\
\hline & & 1992 & 1993 & 1994 & 1998 \\
\hline \multicolumn{6}{|l|}{ ECAR } \\
\hline .......................................... & Investor-Owned & * & 40 & 59 & 246 \\
\hline 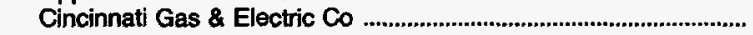 & Investor-Owned & 9 & 6 & 47 & 244 \\
\hline 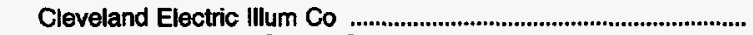 & Investor-Owned & 1 & 19 & 35 & 230 \\
\hline 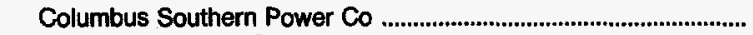 & Investor-Owned & 16 & 26 & 50 & 174 \\
\hline Consumers Power $C_{0}$ & Investor-Owned & 117 & 280 & 596 & 1,456 \\
\hline 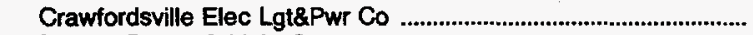 & Publicly Owned & - & & & \\
\hline 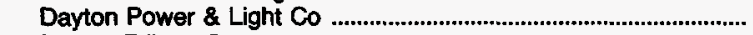 & Investor-Owned & 25 & 90 & 90 & 206 \\
\hline 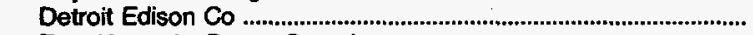 & Investor-Owned & 14 & 149 & 76 & 262 \\
\hline 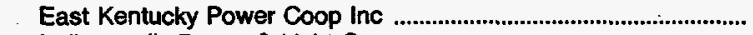 & Cooperative & 3 & -1 & -1 & 55 \\
\hline 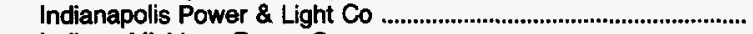 & Investor-Owned & 34 & 41 & 72 & 177 \\
\hline 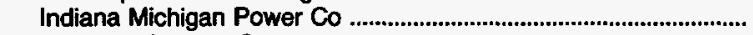 & Investor-Owned & 5 & 4 & 10 & 103 \\
\hline 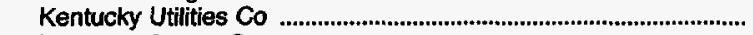 & Investor-Owned & 53 & 37 & 42 & 61 \\
\hline 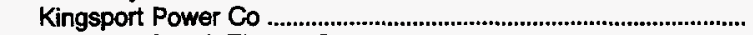 & Investor-Owned & * & 4 & 4 & 20 \\
\hline 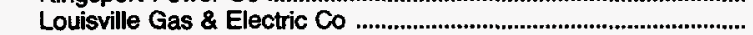 & Investor-Owned & 0 & 16 & 30 & 35 \\
\hline 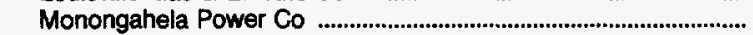 & Investor-Owned & 201 & 217 & 220 & 253 \\
\hline 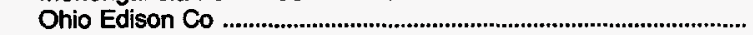 & Investor-Owned & 44 & 61 & 134 & 526 \\
\hline Ohio Power Co & Investor-Owned & 18 & 18 & 40 & 207 \\
\hline 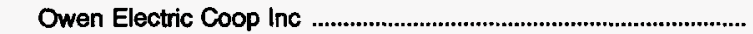 & Cooperative & * & 1 & 1 & 3 \\
\hline  & Investor-Owned & 19 & 0 & 4 & 20 \\
\hline Potomac Edison Co ............ & Investor-Owned & 286 & 314 & 341 & 505 \\
\hline 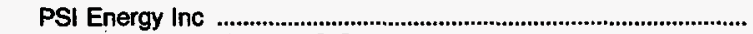 & Investor-Owned & 25 & 141 & 289 & 941 \\
\hline 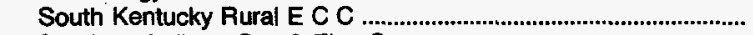 & Cooperative & * & - & - & - \\
\hline 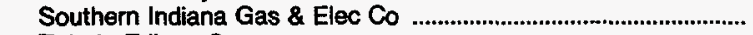 & Investor-Owned & 4 & 16 & 36 & 132 \\
\hline Toledo Edison Co & Investor-Owned & 2 & 16 & 24 & 117 \\
\hline Utilities Dist-Western IN REMC & Cooperative & 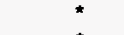 & 12 & 2 & 6 \\
\hline  & Publicly Owned & * & * & * & * \\
\hline West Penn Power Co & Investor-Owned & 250 & 263 & 272 & 324 \\
\hline 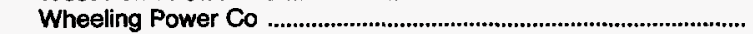 & Investor-Owned & 2 & 1 & 2 & 12 \\
\hline 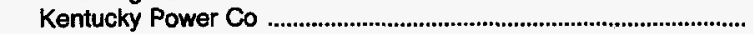 & Investor-Owned & - & 6 & $\overline{7}$ & 39 \\
\hline 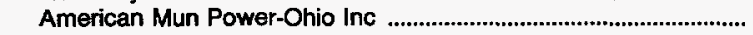 & Publicly Owned & 1 & * & * & \\
\hline 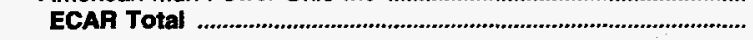 & & 1,129 & 1,779 & 2,483 & 6,355 \\
\hline \multicolumn{6}{|l|}{ ERCOT } \\
\hline 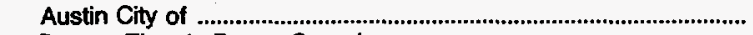 & Publicly Owned & 407 & 448 & 513 & 778 \\
\hline 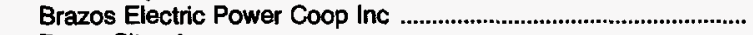 & Cooperative & 2 & 6 & 2 & 16 \\
\hline Bryan City of & Publicly Owned & 6 & 8 & 10 & 18 \\
\hline 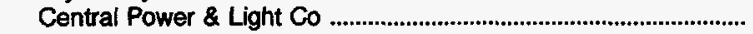 & Investor-Owned & 84 & 185 & 203 & 343 \\
\hline Denton City of & Publicly Owned & 1 & 0 & 0 & 0 \\
\hline Georgetown City of & Publicly Owned & - & 1 & 1 & \\
\hline Greenville Electric Util Sys ................................................................... & Publicly Owned & 1 & - & * & 4 \\
\hline 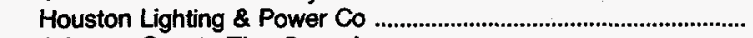 & Investor-Owned & 71 & 130 & 174 & 312 \\
\hline 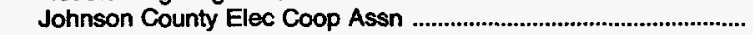 & Cooperative & * & 4 & 0 & 0 \\
\hline 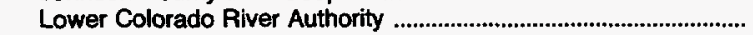 & Publicly Owned & 89 & 105 & 112 & 205 \\
\hline 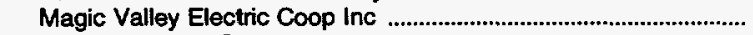 & Cooperative & - & 2 & 2 & 4 \\
\hline 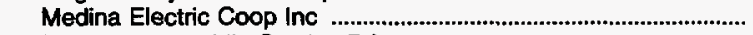 & Cooperative & 4 & 0 & 0 & 0 \\
\hline 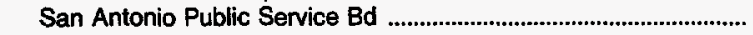 & Publicly Owned & 129 & 0 & 0 & 0 \\
\hline 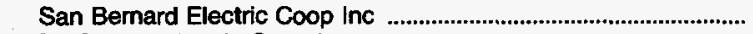 & Cooperative & & * & & \\
\hline 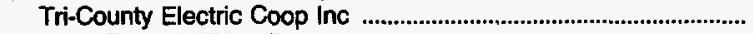 & Cooperative & 1 & 1 & 2 & 3 \\
\hline West Texas Utilities Co & Investor-Owned & 61 & 62 & 45 & 102 \\
\hline San Marcos City of & Publicly Owned & 11 & 11 & * & $*$ \\
\hline 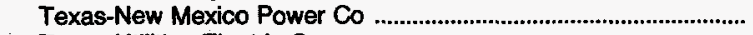 & Investor-Owned & 37 & 105 & 132 & 223 \\
\hline 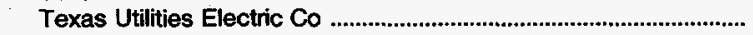 & Investor-Owned & 110 & 1,221 & 1,288 & 1,288 \\
\hline 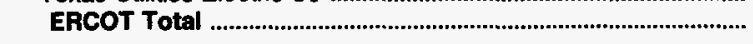 & & 1,013 & 2,288 & 2,483 & $\mathbf{3 , 2 9 5}$ \\
\hline \multicolumn{6}{|l|}{ MAAC } \\
\hline A\& & rative & 0 & 1 & 1 & \\
\hline Atlantic City Electric Co & Investor-Owned & 55 & 65 & 59 & -5 \\
\hline 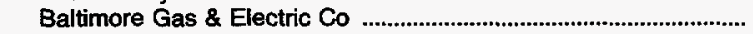 & Investor-Owned & 116 & 190 & 258 & 498 \\
\hline Conowingo Power $\mathrm{Co}$ & Investor-Owned & 3 & 4 & 7 & 13 \\
\hline 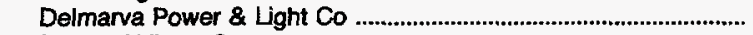 & Investor-Owned & 31 & 46 & 43 & 115 \\
\hline Easton Utilities Comm & Publicly Owned. & - & * & 2 & 12 \\
\hline 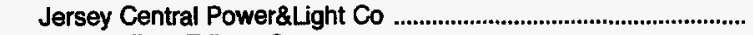 & Investor-Owned & 67 & 106 & 163 & 277 \\
\hline Metropolitan Edison Co & Investor-Owned & 277 & 81 & 82 & 140 \\
\hline 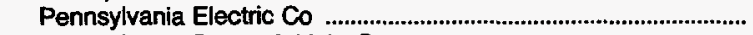 & Investor-Owned & 59 & 75 & 92 & 137 \\
\hline  & Investor-Owned & 20 & 29 & 25 & 40 \\
\hline 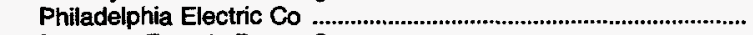 & Investor-Owned & 52 & 60 & 67 & 71 \\
\hline 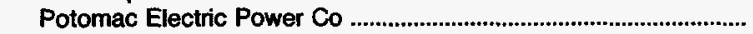 & Investor-Owned & 214 & 431 & 774 & 2,391 \\
\hline 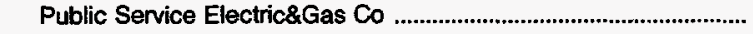 & Investor-Owned & 58 & 57 & 604 & 1,500 \\
\hline
\end{tabular}

See footnotes at end of table. 
Table 9. U.S. Electric Utility Energy Savings by North American Electric Reliability Council Region and Hawali by Class of Ownership, 1992, 1993, 1994 and 1998 (Million Kilowatthours) (Continued)

\begin{tabular}{|c|c|c|c|c|c|}
\hline \multirow{2}{*}{$\begin{array}{c}\text { North American Electric Rellabillty } \\
\text { Council Region and Hawail / } \\
\text { Electric Utllity }\end{array}$} & \multirow{2}{*}{$\begin{array}{c}\text { Class of } \\
\text { Ownership }\end{array}$} & \multicolumn{2}{|c|}{ Historical Savings } & \multicolumn{2}{|c|}{ Projected Savings } \\
\hline & & 1992 & 1993 & 1994 & 1998 \\
\hline
\end{tabular}

MAAC (Continued)

Southern Maryland El Coop Inc

UGI Utilities Inc

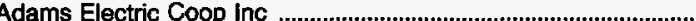

Bedford Rural Elec Coop Inc

Southwest Central R E C Corp

MAAC Total

MAIN

Central Illinois Light Co

Coles-Moultrie Electric Coop

Columbia City of .

Commonwealth Edison Co

Cuivre River Electric Coop Inc

Eastern Illini Electric Coop

Illinois Power Co

Madison Gas \& Electric Co

Manitowoc Public Utilities

Marshfield City of

Southeastern IL Elec Coop Inc

Southwestern Electric Coop Inc

Springfield City of

Union Electric Co

Wisconsin Electric Power Co...................

Wisconsin Power \& Light Co

Wisconsin Public Power Inc Sys

Wisconsin Public Service Corp

MAIN Total

MAPP(U.S.)

Ames City of

Anoka Electric Coop

Austin City of

Barron Electric Coop

Beatrice City of .

Cedar Falls City of

Central lowa Power Coop ..

Central Power Elec Coop Inc

Clark Electric Coop

Coop Power Assn

Cornhusker Public Power Dist

Fairmont Public Utilities Comm

Freeborn-Mower Electric Coop

Grant-Lafayette Electric

IES Utilities Inc

Interstate Power Co

lowa Electric Light \& Power $\mathrm{Co}$

lowa Lakes Electric Coop

lowa Southern Utilities Co .

lowa-Illinois Gas\&Electric Co

Lincoln Electric System

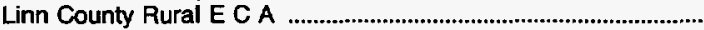

Marshall City of

Nodak Electric Coop Inc

Midland Power Coop

Maquoketa Valley Rrl Elec Coop

Minnesota Power \& Light Co

Minnkota Power Coop Inc

Moorhead City of

Muscatine City of

North Platte City of

Northern States Power Co

Northern States Power Co

Northwest lowa Power Coop

Northwestern Public Service Co

Oakdale Electric Coop

Omaha Public Power District

Otter Tail Power Co
Cooperative

Investor-Owned

Cooperative

Cooperative

Cooperative

Investor-Owned

Cooperative

Publicly Owned

Investor-Owned

Cooperative

Cooperative

Investor-Owned

Investor-Owned

Publicly Owned

Publicly Owned

Cooperative

Cooperative

Publicly Owned

Investor-Owned

Investor-Owned

Investor-Owned

Publicly Owned

Investor-Owned

Publicly Owned Cooperative

Publicly Owned

Cooperative

Publicly Owned

Publicly Owned

Cooperative

Cooperative

Cooperative

Cooperative

Publicly Owned

Publicly Owned

Cooperative

Cooperative

Investor-Owned

Investor-Owned

Investor-Owned

Cooperative

Investor-Owned

Investor-Owned

Publicly Owned

Cooperative

Publicly Owned

Cooperative

Cooperative

Cooperative

Investor-Owned

Cooperative

Publicly Owned

Publicly Owned

Publicly Owned

Investor-Owned

Investor-Owned

Cooperative

Investor-Owned

Cooperative

Publicly Owned

Investor-Owned



4
$* \quad 1$
0
150

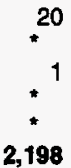

150

1

-

5,340

\begin{tabular}{rr}
\multicolumn{1}{r}{$\begin{array}{r}* \\
*\end{array}$} & $*$ \\
$*$ & 4 \\
2 & 2 \\
2 & 0 \\
0 & 3 \\
62 & 34 \\
7 & 90 \\
1 & 9 \\
4 & 2 \\
28 & 124 \\
5 & 29 \\
1 & 6 \\
751 & 0 \\
133 & 1,286 \\
3 & 197 \\
210 & 16 \\
1,212 & 322 \\
& 2,125
\end{tabular}

$\begin{array}{rr}0 & 20 \\ 5 & 9 \\ 0 & 0 \\ 0 & 0 \\ 3 & 3 \\ 34 & 34 \\ 118 & 249 \\ 2 & 1 \\ 4 & 9 \\ 206 & 887 \\ 26 & 36 \\ 7 & 13 \\ 7 & 282 \\ 1,392 & 1,751 \\ 293 & 648 \\ 40 & 254 \\ 418 & 819 \\ 2,555 & 5,015\end{array}$

\begin{tabular}{|c|c|c|c|}
\hline 1 & 1 & 5 & 19 \\
\hline 8 & - & - & \\
\hline * & * & 1 & \\
\hline-3 & * & * & $\bullet$ \\
\hline * & * & $\star$ & • \\
\hline * & 19 & 20 & 2 \\
\hline - & 48 & 52 & 72 \\
\hline 7 & * & 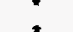 & \\
\hline-7 & 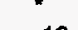 & * & " \\
\hline${ }_{*}^{14}$ & ${ }_{*}^{10}$ & 31 & 115 \\
\hline * & 1 & 1 & \\
\hline- & * & $\star$ & \\
\hline-8 & 1 & 1 & 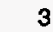 \\
\hline- & 21 & 80 & 380 \\
\hline 16 & 34 & 62 & 87 \\
\hline-3 & - & - & - \\
\hline 32 & 38 & 20 & 30 \\
\hline 2 & - & - & - \\
\hline- & 0 & 23 & 88 \\
\hline 12 & 14 & 15 & 15 \\
\hline * & - & - & \\
\hline * & & * & $*$ \\
\hline 1 & 2 & 3 & \\
\hline 3 & 6 & 7 & \\
\hline * & - & - & \\
\hline 18 & 27 & 122 & 15 \\
\hline 0 & 7 & 0 & \\
\hline * & * & 1 & \\
\hline * & 2 & 3 & \\
\hline 1 & * & $*$ & $*$ \\
\hline 197 & 86 & 289 & 460 \\
\hline 507 & 1,009 & 1,032 & 2,416 \\
\hline 4 & 6 & 6 & \\
\hline * & 0 & 0 & \\
\hline-8 & * & * & \\
\hline 1 & 1 & 14 & \\
\hline 10 & 38 & 39 & \\
\hline
\end{tabular}

See footnotes at end of table. 
Table 9. U.S. Electric Utility Energy Savings by North American Electric Reliability Council Region and Hawaii by Class of Ownership, 1992, 1993, 1994 and 1998 (Million Kilowatthours) (Continued)

\begin{tabular}{|c|c|c|c|c|c|}
\hline \multirow{2}{*}{$\begin{array}{c}\text { North American Electric Reliability } \\
\text { Councll Region and Hawail / } \\
\text { Electric Utility }\end{array}$} & \multirow{2}{*}{$\begin{array}{l}\text { Class of } \\
\text { Ownership }\end{array}$} & \multicolumn{2}{|c|}{ Hlstorical Savings } & \multicolumn{2}{|c|}{ Projected Savings } \\
\hline & & 1992 & 1993 & 1994 & 1998 \\
\hline \multicolumn{6}{|l|}{ MAPP(U.S.) (Continued) } \\
\hline Owatonna City of .......... & Publicly Owned & 1 & 1 & * & * \\
\hline People's Coop Power Assn & Cooperative & -3 & * & * & * \\
\hline Pella City of & Publicly Owned & - & 2 & 2 & 2 \\
\hline 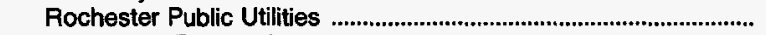 & Publicly Owned & 1 & * & 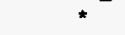 & 1 \\
\hline 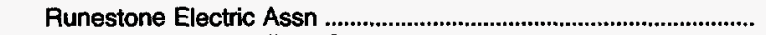 & Cooperative & 1 & 2 & 2 & 2 \\
\hline 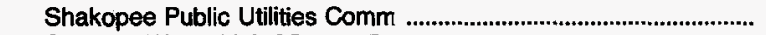 & Publicly Owned & 0 & * & * & $*$ \\
\hline 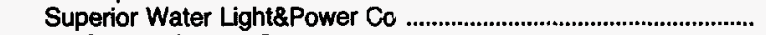 & Investor-Owned & 8 & 2 & 20 & 36 \\
\hline  & Cooperative & -8 & * & 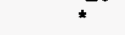 & 1 \\
\hline 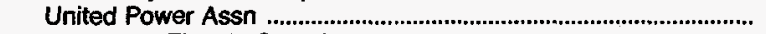 & Cooperative & 15 & 23 & 29 & 33 \\
\hline 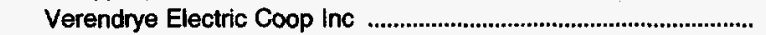 & Cooperative & * & * & $\star$ & * \\
\hline 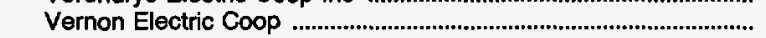 & Cooperative & - & * & * & * \\
\hline 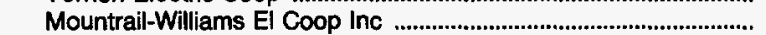 & Cooperative & *. & 14 & 15 & 16 \\
\hline 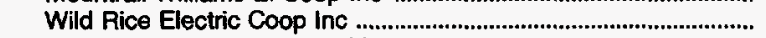 & Cooperative & 50 & 56 & 54 & 58 \\
\hline 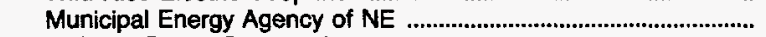 & Publicly Owned & 0 & 1 & 1 & 3 \\
\hline 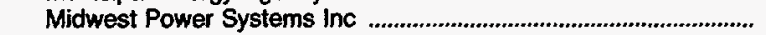 & Investor-Owned & 75 & 105 & 123 & 291 \\
\hline  & Cooperative & 1 & & 2 & \\
\hline 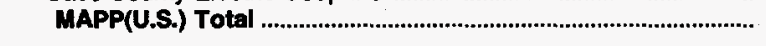 & & 940 & 1,581 & 2,075 & 4,415 \\
\hline \multicolumn{6}{|l|}{ NPCC(U.S.) } \\
\hline 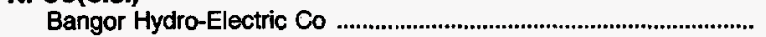 & Investor-Owned & 35 & 38 & 45 & 71 \\
\hline 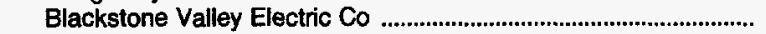 & Investor-Owned & 42 & 61 & 55 & 87 \\
\hline 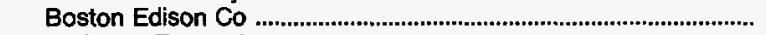 & Investor-Owned & 336 & 382 & 133 & 55 \\
\hline 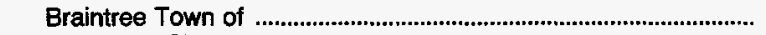 & Publicly Owned & 3 & * & * & 20 \\
\hline Burlington City of & Publicly Owned & 18 & $\mathbf{9}$ & 9 & 9 \\
\hline Cambridge Electric Light $C_{0}$ & Investor-Owned & 49 & 71 & 71 & 71 \\
\hline 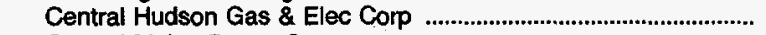 & Investor-Owned & 75 & 97 & 122 & 265 \\
\hline 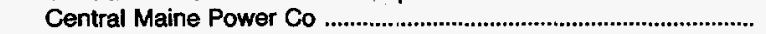 & Investor-Owned & 352 & 390 & 449 & 355 \\
\hline Central Vermont Pub Serv Corp & Investor-Owned & 13 & 41 & 55 & 53 \\
\hline 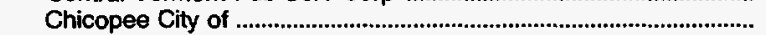 & Publicly Owned & 3 & 4 & 3 & 4 \\
\hline Citizens Utilities Co. & Investor-Owned & 2 & 2 & 8 & 24 \\
\hline 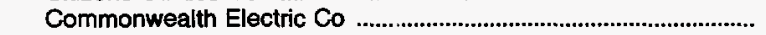 & Investor-Owned & 109 & $12 \overline{1}$ & 120 & 120 \\
\hline 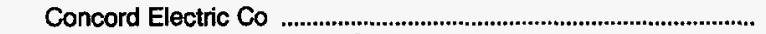 & Investor-Owned & * & 1 & 3 & 13 \\
\hline Connecticut Light \& Power Co & Investor-Owned & 1,035 & 1,160 & 1,193 & 1,490 \\
\hline 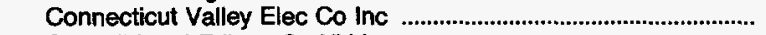 & Investor-Owned & * & 2 & 1 & \\
\hline 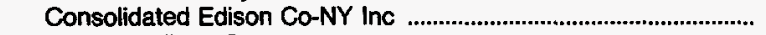 & Investor-Owned & 638 & 498 & 1,634 & 2,585 \\
\hline 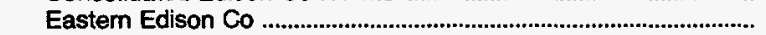 & Investor-Owned & 62 & 94 & 11 & 179 \\
\hline 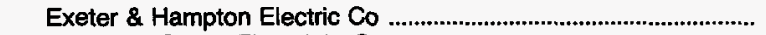 & Investor-Owned & & 1 & 4 & 13 \\
\hline Fitchburg Gas \& Elec Light $\mathrm{Co}_{0}$ & Investor-Owned & 8 & 8 & 9 & 20 \\
\hline Green Mountain Power Corp ............................................................ & Investor-Owned & 10 & 30 & 46 & 108 \\
\hline 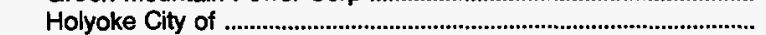 & Publicly Owned & & & & $*$ \\
\hline 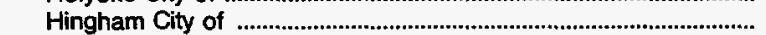 & Publicly Owned & * & 4 & 4 & \\
\hline 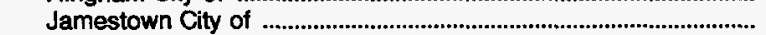 & Publicly Owned & * & $\star$ & 6 & \\
\hline 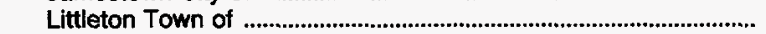 & Publicly Owned & -- & * & * & * \\
\hline 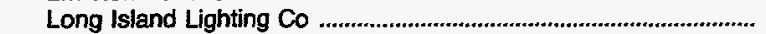 & Investor-Owned & 460 & 580 & 686 & 1,129 \\
\hline 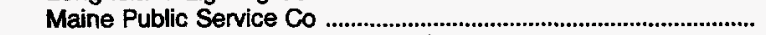 & investor-Owned & 6 & 5 & 7 & \\
\hline 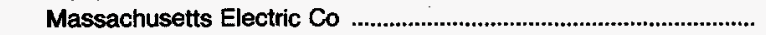 & Investor-Owned & 345 & 549 & 691 & 1,374 \\
\hline 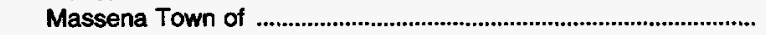 & Publicly Owned & * & 1 & 1 & \\
\hline 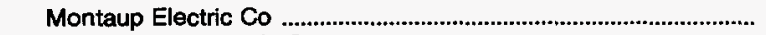 & Investor-Owned & 111 & 170 & 174 & 299 \\
\hline 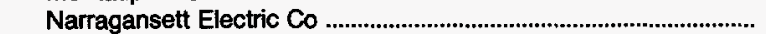 & Investor-Owned & 127 & 181 & 214 & 320 \\
\hline 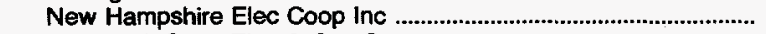 & Cooperative & 6 & 7 & 8 & 43 \\
\hline New York State Elec \& Gas Corp ................................................................. & Investor-Owned & 139 & 695 & 589 & 810 \\
\hline 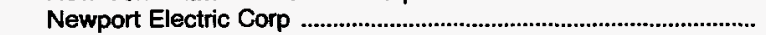 & Investor-Owned & 8 & 15 & 17 & 33 \\
\hline Niagara Mohawk Power Corp ………………………………….. & Investor-Owned & 475 & 737 & 965 & 1,848 \\
\hline 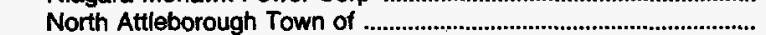 & Publicly Owned & $\cdots$ & - & - & - \\
\hline 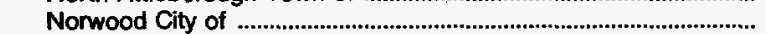 & Publicly Owned & - & 1 & * & 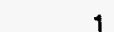 \\
\hline 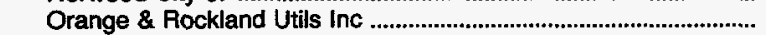 & Investor-Owned & 123 & 167 & 203 & 341 \\
\hline Power Authority of State of NY .............. & Publicly Owned & 42 & 81 & 167 & 354 \\
\hline 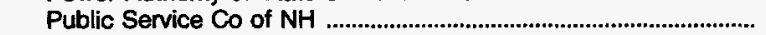 & Investor-Owned & 0 & 1 & 6 & 44 \\
\hline 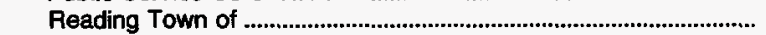 & Publicly Owned & * & * & * & \\
\hline 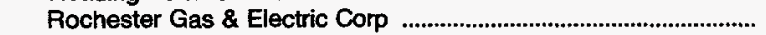 & Investor-Owned & 127 & 183 & 202 & 375 \\
\hline 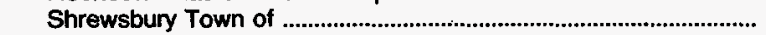 & Publicly Owned & 1 & 1 & 1 & \\
\hline 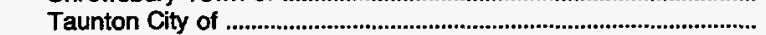 & Publicly Owned & 11 & 16 & 8 & 10 \\
\hline United Illuminating $\mathrm{Co}$ & Investor-Owned & 75 & 137 & 151 & 280 \\
\hline 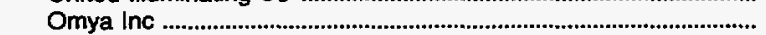 & Investor-Owned & -- & " & 8 & 14 \\
\hline Western Massachusetts Elec Co & Investor-Owned & 183 & 202 & 219 & 347 \\
\hline 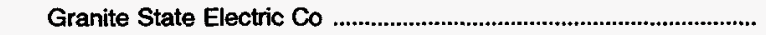 & Investor-Owned & 21 & 28 & 34 & 67 \\
\hline 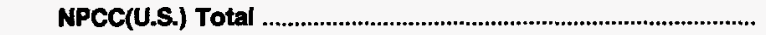 & & 5,049 & 6,769 & 8,332 & 13,263 \\
\hline
\end{tabular}

See footnotes at end of table. 
Table 9. U.S. Electric Utility Energy Savings by North American Electric Reliability Council Region and Hawaii by Class of Ownership, 1992, 1993, 1994 and 1998 (Million Kilowatthours) (Continued)

\begin{tabular}{|c|c|c|c|c|c|}
\hline \multirow{2}{*}{$\begin{array}{l}\text { North American Electric Reliability } \\
\text { Council Region and Hawail / } \\
\text { Electric Utility }\end{array}$} & \multirow{2}{*}{$\begin{array}{l}\text { Class of } \\
\text { Ownership }\end{array}$} & \multicolumn{2}{|c|}{ Historical Savings } & \multicolumn{2}{|c|}{ Projected Savings } \\
\hline & & 1992 & 1993 & 1994 & 1998 \\
\hline \multicolumn{6}{|l|}{ SERC } \\
\hline 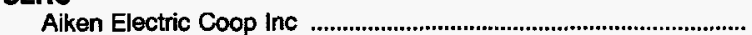 & Cooperative & 3 & 1 & 1 & 2 \\
\hline 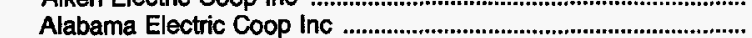 & Cooperative & 18 & 24 & 4 & 4 \\
\hline Alabama Power Co & Investor-Owned & 453 & 449 & 534 & 699 \\
\hline Albemarle City of & Publicly Owned & - & $\star$ & 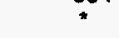 & $*$ \\
\hline 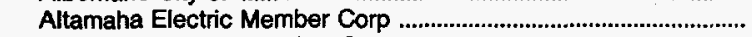 & Cooperative & * & * & * & * \\
\hline 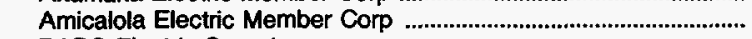 & Cooperative & * & * & * & * \\
\hline 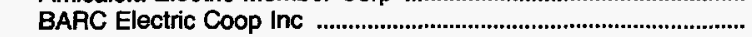 & Cooperative & 0 & * & * & * \\
\hline 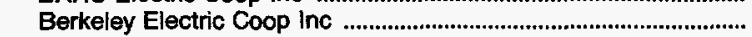 & Cooperative & 4 & 4 & 4 & 6 \\
\hline 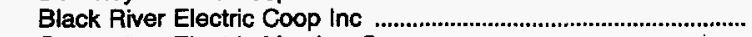 & Cooperative & 4 & 2 & 2 & 3 \\
\hline Canoochee Electric Member Corp & Cooperative & * & * & $*^{-}$ & * \\
\hline Carolina Power \& Light Co & Investor-Owned & 1,824 & 2,062 & 2,126 & 2,621 \\
\hline 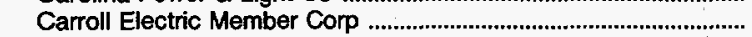 & Cooperative & 1 & 1 & 1 & \\
\hline Central Georgia El Member Corp & Cooperative & 2 & 2 & 3 & 5 \\
\hline Central Electric Member Corp & Cooperative & 2 & $\star^{2}$ & 2 & 4 \\
\hline Coast Electric Power Assn & Cooperative & * & * & $\star^{2}$ & * \\
\hline Coastal Electric Member Corp & Cooperative & - & 1 & 1 & 1 \\
\hline  & Cooperative & 14 & 16 & 2 & 2 \\
\hline Community Electric Coop & Cooperative & - & $*$ & $*^{2}$ & * \\
\hline Coweta-Fayette EI Member Corp & Cooperative & 5 & 57 & 58 & 64 \\
\hline 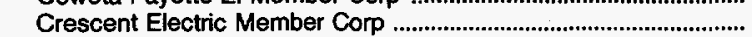 & Cooperative & $*$ & 1 & 1 & 1 \\
\hline 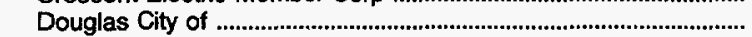 & Publicly Owned & 1 & 1 & 1 & 2 \\
\hline Duke Power Co & Investor-Owned & 50 & 74 & 27 & 1,420 \\
\hline Excelsior Electric Member Corp & Cooperative & $*$ & * & $*$ & \\
\hline 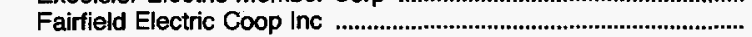 & Cooperative & * & * & * & $\cdot$ \\
\hline 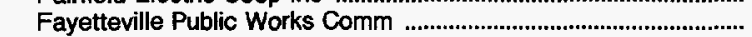 & Publicly Owned & * & * & * & * \\
\hline Fitzgerald Wtr Lgt \& Bond Comm & Publicly Owned & * & * & * & * \\
\hline Flint Electric Membership Corp & Cooperative & 1 & 4 & 3 & 3 \\
\hline 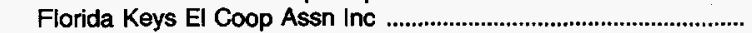 & Cooperative & $\star$ & * & $*$ & * \\
\hline Florida Power \& Light Co & Investor-Owned & 2,559 & 2,738 & 2,888 & 3,273 \\
\hline Florida Power Corp & Investor-Owned & 922 & 989 & 989 & 989 \\
\hline Fort Pierce Utilities Auth & Publicly Owned & 1 & 1 & 1 & \\
\hline 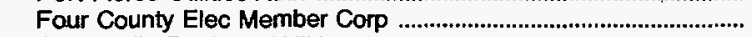 & Cooperative & 2 & 1 & 1 & \\
\hline 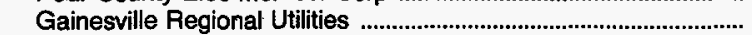 & Publicly Owned & $5 \overline{5}$ & 65 & 66 & 78 \\
\hline Gastonia City of & Publicly Owned & 1 & - & - & - \\
\hline Georgia Power Co & Investor-Owned & 61 & 134 & 175 & 476 \\
\hline Grady County Elec Member Corp & Cooperative & $*$ & 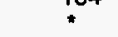 & $*$ & $*$ \\
\hline Greenville Utilities Comm & Publicly Owned & 13 & 14 & 15 & 19 \\
\hline Gulf Power Co & Investor-Owned & 401 & 418 & 438 & 530 \\
\hline 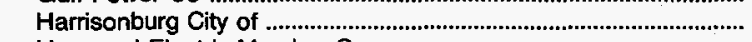 & Publicly Owned & 0 & 0 & 2 & \\
\hline 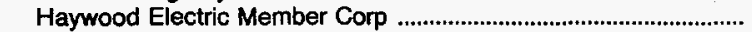 & Cooperative & $*$ & $*$ & * & \\
\hline 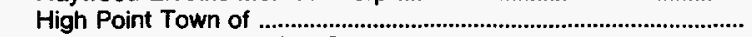 & Publicly Owned & * & 0 & 0 & \\
\hline Jackson Electric Member Corp & Cooperative & 10 & 11 & 11 & 13 \\
\hline 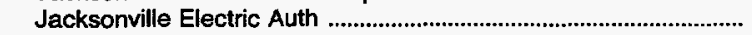 & Publicly Owned & 104 & 108 & 116 & 150 \\
\hline Jefferson Electric Member Corp & Cooperative & $*$ & $*$ & 1 & \\
\hline 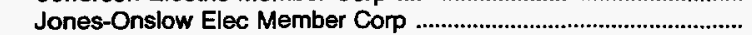 & Cooperative & 2 & 2 & 2 & \\
\hline Kissimmee Utility Authority & Publicly Owned & 6 & 4 & 5 & 8 \\
\hline Lakeland City of & Publicly Owned & 1 & 1 & 1 & \\
\hline 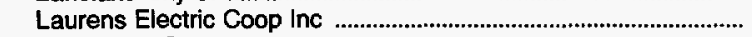 & Cooperative. & * & * & * & * \\
\hline Laurinburg City of & Publicly Owned & - & * & * & * \\
\hline Lawrenceville City of & Publicly Owned & * & * & * & * \\
\hline 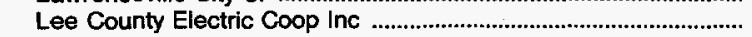 & Cooperative & 19 & 21 & 20 & 29 \\
\hline 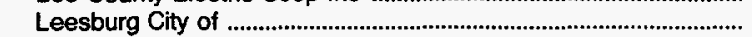 & Publicly Owned & $*$ & $*$ & & \\
\hline 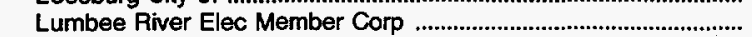 & Cooperative & 2 & 2 & 2 & \\
\hline Lumberton City of & Publicly Owned & $*$ & * & & \\
\hline Lynches River Elec Coop Inc & Cooperative & 2 & 1 & 1 & \\
\hline Manassas City of & Publicly Owned & 4 & 3 & 4 & \\
\hline Marietta City of & Publicly Owned & $\star+$ & 2 & $0^{4}$ & \\
\hline Mecklenburg Electric Coop Inc & Cooperative & * & 6 & 1 & \\
\hline 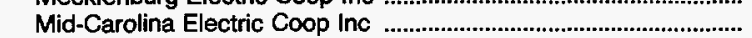 & Cooperative & 6 & 2 & 3 & \\
\hline Mississippi Power Co & Investor-Owned & - & 1 & 1 & \\
\hline Mitchell Electric Member Corp & Cooperative & & & • & \\
\hline 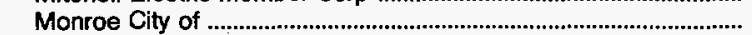 & Publicly Owned & * & * & 1 & \\
\hline Morganton City of & Publicly Owned & 7 & - & - & \\
\hline 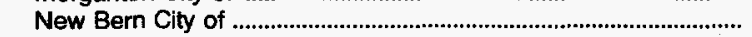 & Publicly Owned & * & * & * & * \\
\hline Newberry City of & Publicly Owned & $*$ & 0 & 0 & \\
\hline 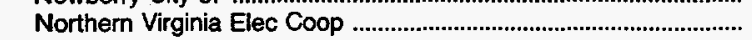 & Cooperative & 3 & 1 & 1 & \\
\hline 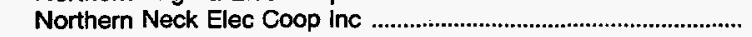 & Cooperative & * & * & $*$ & * \\
\hline
\end{tabular}

See footnotes at end of tabie. 
Table 9. U.S. Electric Utility Energy Savings by North American Electric Reliability Council Region and Hawail by Class of Ownership, 1992, 1993, 1994 and 1998

(Million Kilowatthours) (Continued)

\begin{tabular}{|c|c|c|c|c|c|}
\hline \multirow{2}{*}{$\begin{array}{c}\text { North American Electric Reliability } \\
\text { Councll Region and Hawail / } \\
\text { Electric Utility }\end{array}$} & \multirow{2}{*}{$\begin{array}{l}\text { Class of } \\
\text { Ownership }\end{array}$} & \multicolumn{2}{|c|}{ Historical Savings } & \multicolumn{2}{|c|}{ Projected Savings } \\
\hline & & 1992 & 1993 & 1994 & 1998 \\
\hline 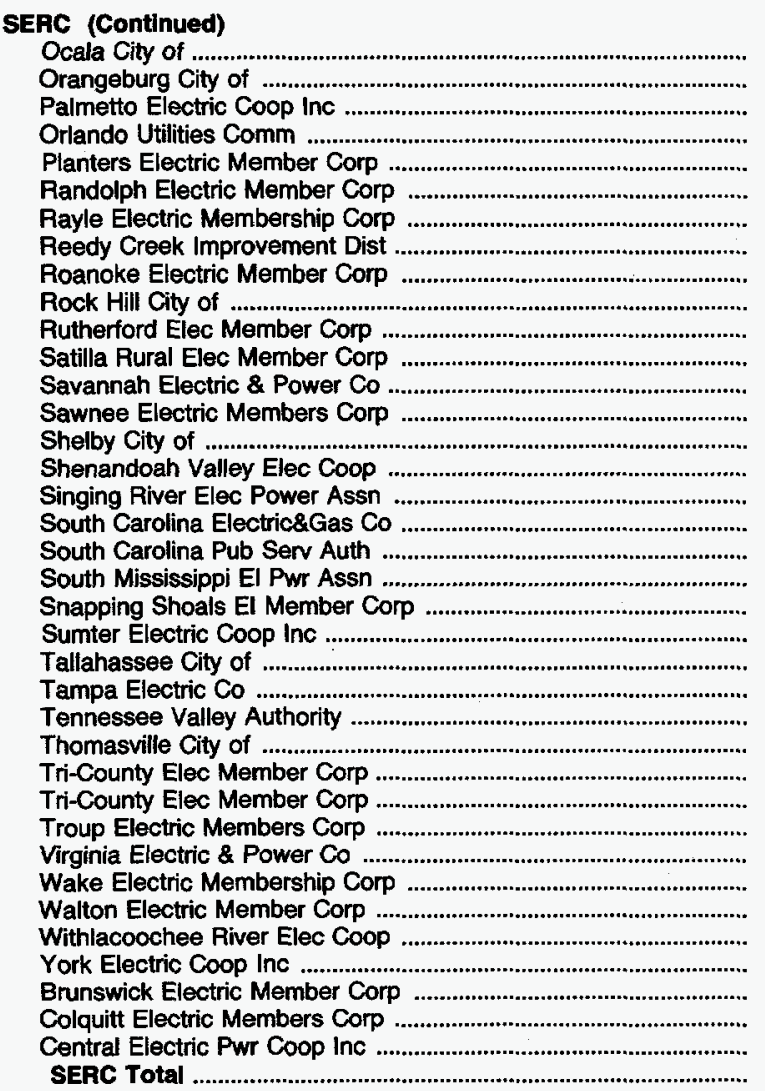 & $\begin{array}{l}\text { Publicly Owned } \\
\text { Publicly Owned } \\
\text { Cooperative } \\
\text { Publicly Owned } \\
\text { Cooperative } \\
\text { Cooperative. } \\
\text { Cooperative } \\
\text { Publicly Owned } \\
\text { Cooperative } \\
\text { Publicly Owned } \\
\text { Cooperative } \\
\text { Cooperative } \\
\text { Investor-Owned } \\
\text { Cooperative } \\
\text { Publicly Owned } \\
\text { Cooperative } \\
\text { Cooperative } \\
\text { Investor-Owned } \\
\text { Publicly Owned } \\
\text { Cooperative } \\
\text { Cooperative } \\
\text { Cooperative } \\
\text { Publicly Owned } \\
\text { Investor-Owned } \\
\text { Federal } \\
\text { Publicly Owned } \\
\text { Cooperative } \\
\text { Cooperative } \\
\text { Cooperative } \\
\text { Investor-Owned } \\
\text { Cooperative } \\
\text { Cooperative } \\
\text { Cooperative } \\
\text { Cooperative } \\
\text { Cooperative } \\
\text { Cooperative } \\
\text { Cooperative }\end{array}$ & $\begin{array}{r}* \\
1 \\
60 \\
0 \\
0 \\
* \\
* \\
- \\
* \\
* \\
* \\
* \\
* \\
* \\
125 \\
20 \\
8 \\
* \\
16 \\
68 \\
183 \\
3,239 \\
20 \\
* \\
0 \\
2 \\
136 \\
2 \\
* \\
* 1 \\
* \\
* \\
1 \\
36 \\
10,492\end{array}$ & $\begin{array}{r}* \\
* 1 \\
74 \\
* \\
* \\
5 \\
0 \\
1 \\
0 \\
* \\
1 \\
1 \\
-1 \\
1 \\
179 \\
24 \\
13 \\
0 \\
17 \\
83 \\
162 \\
3,266 \\
19 \\
- \\
* 0 \\
160 \\
3 \\
* \\
2 \\
1 \\
* \\
2 \\
14 \\
11,264\end{array}$ & $\begin{array}{r}3 \\
1 \\
7 \\
73 \\
* \\
* \\
6 \\
0 \\
1 \\
16 \\
* \\
11 \\
1 \\
2 \\
3 \\
272 \\
31 \\
17 \\
0 \\
18 \\
18 \\
169 \\
3,297 \\
19 \\
- \\
0 \\
184 \\
3 \\
* \\
1 \\
* \\
1 \\
18 \\
11,685\end{array}$ & $\begin{array}{r}6 \\
1 \\
10 \\
102 \\
\vdots \\
\\
6 \\
0 \\
1 \\
20 \\
48 \\
48 \\
2 \\
2 \\
3 \\
394 \\
63 \\
36 \\
0 \\
22 \\
65 \\
205 \\
5,828 \\
20 \\
* \\
0 \\
229 \\
3 \\
1 \\
* \\
1 \\
* \\
1 \\
32 \\
17,536\end{array}$ \\
\hline 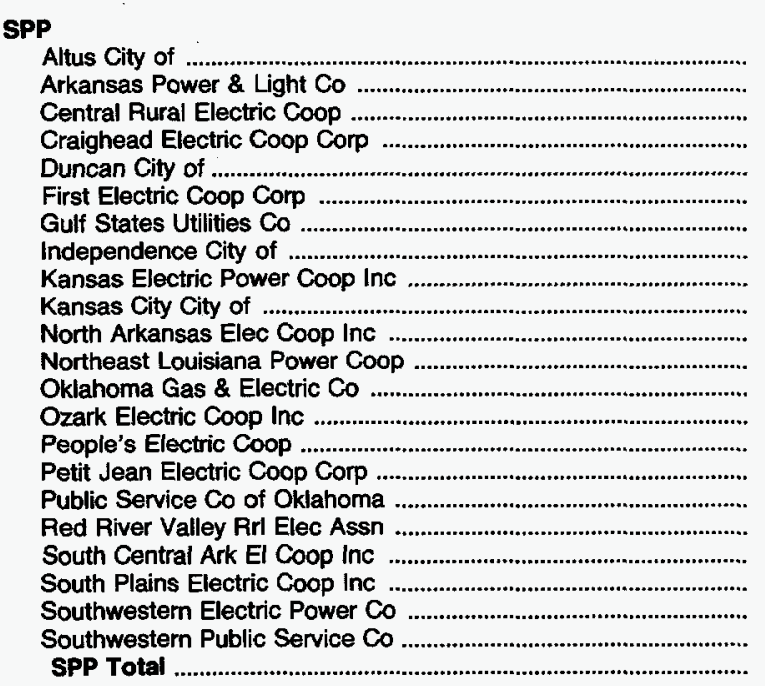 & $\begin{array}{l}\text { Publicly Owned } \\
\text { Investor-Owned } \\
\text { Cooperative } \\
\text { Cooperative } \\
\text { Publicly Owned } \\
\text { Cooperative } \\
\text { Investor-Owned } \\
\text { Publicly Owned } \\
\text { Cooperative } \\
\text { Publicly Owned } \\
\text { Cooperative } \\
\text { Cooperative } \\
\text { Investor-Owned } \\
\text { Cooperative } \\
\text { Cooperative } \\
\text { Cooperative } \\
\text { Investor-Owned } \\
\text { Cooperative } \\
\text { Cooperative } \\
\text { Cooperative } \\
\text { Investor-Owned } \\
\text { Investor-Owned }\end{array}$ & $\begin{array}{r}* 0 \\
2 \\
2 \\
* \\
* \\
5 \\
20 \\
1 \\
1 \\
22 \\
{ }^{*} \\
-116 \\
7 \\
* 7 \\
* \\
0 \\
8 \\
45 \\
21 \\
23 \\
273\end{array}$ & 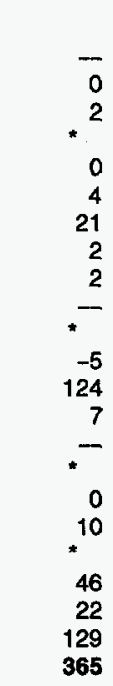 & $\begin{array}{r}- \\
12 \\
2 \\
* \\
0 \\
4 \\
21 \\
2 \\
2 \\
-5 \\
* \\
-6 \\
124 \\
9 \\
-\square \\
10 \\
10 \\
2 \\
70 \\
25 \\
159 \\
458\end{array}$ & $\begin{array}{r}-49 \\
3 \\
* \\
0 \\
4 \\
30 \\
4 \\
2 \\
- \\
6 \\
124 \\
15 \\
- \\
- \\
67 \\
11 \\
3 \\
90 \\
31 \\
255 \\
695\end{array}$ \\
\hline
\end{tabular}

See footnotes at end of table. 
Table 9. U.S. Electric Utility Energy Savings by North American Electric Reliability Council Region and Hawaii by Class of Ownership, 1992, 1993, 1994 and 1998

(Million Kilowatthours) (Continued)

\begin{tabular}{|c|c|c|c|c|c|}
\hline \multirow{2}{*}{$\begin{array}{l}\text { North American Electric Reliability } \\
\text { Councll Region and Hawall / } \\
\text { Electric Utility }\end{array}$} & \multirow{2}{*}{$\begin{array}{l}\text { Class of } \\
\text { Ownership }\end{array}$} & \multicolumn{2}{|c|}{ Historical Savings } & \multicolumn{2}{|c|}{ Projected Savings } \\
\hline & & 1992 & 1993 & 1994 & 1998 \\
\hline \multicolumn{6}{|l|}{ wscc(U.S.) } \\
\hline 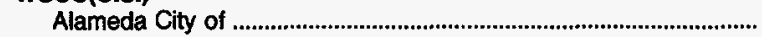 & Publicly Owned & 3 & 6 & 8 & 9 \\
\hline Anaheim City of & Publicly Owned & 2 & $*$ & & * \\
\hline Arizona Electric Pwr Coop Inc & Cooperative & 0 & 2 & 7 & 20 \\
\hline Arizona Public Senvice Co & Investor-Owned & 467 & $49 \overline{3}$ & 567 & 871 \\
\hline Ashland City of & Publicly Owned & $*$ & 0 & 0 & \\
\hline PUD No 1 of Benton County & Publicly Owned & 29 & - & - & - \\
\hline 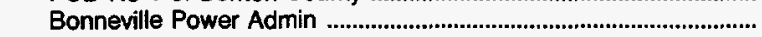 & Federal & 3,583 & 3,685 & 5,441 & 11,842 \\
\hline Bountiful City City of & Publicly Owned & * & + & ( & \\
\hline PUD No 1 of Chelan County & Publicly Owned & 17 & 0 & 35 & 35 \\
\hline El Paso Electric Co & Investor-Owned & 12 & 32 & 37 & 77 \\
\hline Eugene City of & Publicly Owned & 134 & - & - & - \\
\hline Forest Grove City of & Publicly Owned & 3 & 0 & 0 & 0 \\
\hline Idaho Power Co & Investor-Owned & 80 & 87 & 148 & 382 \\
\hline 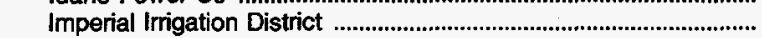 & Publicly Owned & 21 & 48 & 126 & 143 \\
\hline PUD No 1 of Lewis County & Publicly Owned & 1 & - & - & - \\
\hline 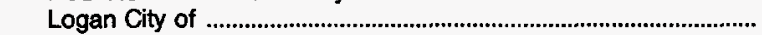 & Publicly Owned & * & - & - & - \\
\hline Longmont City of & Publicly Owned & 27 & 33 & 8 & 9 \\
\hline Los Angeles City of & Publicly Owned & 43 & 210 & 248 & 420 \\
\hline Loveland City of & Publicly Owned & * & * & 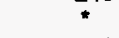 & 1 \\
\hline 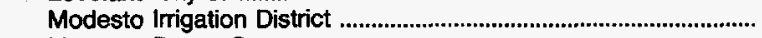 & Publicly Owned & 2 & 5 & 3 & 5 \\
\hline Montana Power Co & Investor-Owned & $5 \overline{9}$ & 118 & 171 & 537 \\
\hline 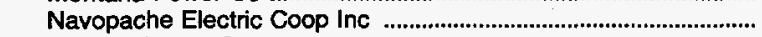 & Cooperative & 1 & 2 & 2 & 2 \\
\hline Nevada Power Co & Investor-Owned & 131 & 150 & 174 & 298 \\
\hline 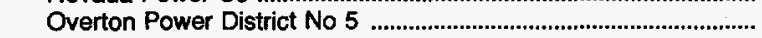 & Publicly Owned & - & $*$ & $\star$ & $*$ \\
\hline Pacific Gas \& Electric Co & Investor-Owned & 1,352 & 1,610 & 2,150 & 2,054 \\
\hline PacifiCorp & Investor-Owned & 263 & 678 & 420 & 629 \\
\hline Palo Alto City of & Publicly Owned & 10 & 10 & 10 & 25 \\
\hline 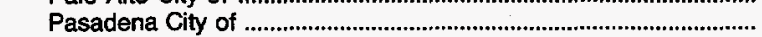 & Publicly Owned & 4 & 7 & 11 & 19 \\
\hline PUD No 2 of Grant County & Publicly Owned & 23 & - & - & 10 \\
\hline Port Angeles City of & Publicly Owned & 9 & - & - & - \\
\hline 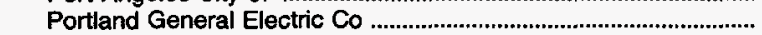 & Investor-Owned & 150 & 313 & 175 & 158 \\
\hline Public Service Co of Colorado & Investor-Owned & 78 & 1,071 & 263 & 804 \\
\hline Puget Sound Power \& Light Co & Investor-Owned & 1,230 & 1,490 & 1,712 & 2,238 \\
\hline Redding City of & Publicly Owned & 1 & 83 & 92 & 130 \\
\hline  & Publicly Owned & 9 & 10 & 11 & 13 \\
\hline Roseville City of & Publicly Owned & * & 1 & 1 & 2 \\
\hline 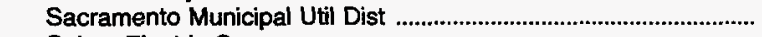 & Publicly Owned & 173 & 269 & 416 & 897 \\
\hline Salem Electric Coop & Cooperative & 9 & - & - & - \\
\hline 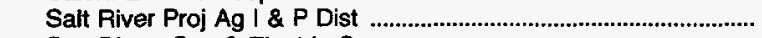 & Publicly Owned & 31 & 41 & 26 & 160 \\
\hline 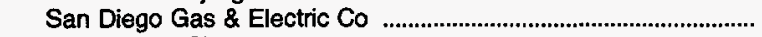 & Investor-Owned & 118 & 134 & 132 & 120 \\
\hline Santa Clara City of & Publicly Owned & 0 & 50 & 50 & 50 \\
\hline Seattle City of & Publicly Owned & 279 & 334 & 430 & 707 \\
\hline Sierra Pacific Power Co & Investor-Owned & 23 & 152 & 177 & 283 \\
\hline 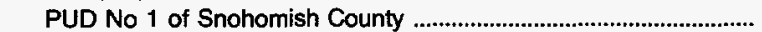 & Publicly Owned & 210 & - & - & - \\
\hline 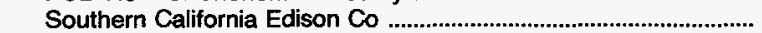 & Investor-Owned & 5,411 & 6,113 & 6,477 & 8,565 \\
\hline Springfield City of & Publicly Owned & 7 & 54 & 7 & 10 \\
\hline 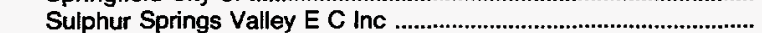 & Cooperative & * & * & 1 & \\
\hline Tacoma City of & Publicly Owned & 165 & 183 & 223 & 380 \\
\hline Trico Electric Coop Inc & Cooperative & - & $\star$ & 0 & 0 \\
\hline 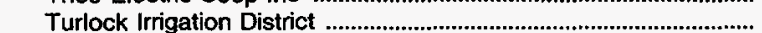 & Publicly Owned & 4 & 5 & 10 & 19 \\
\hline 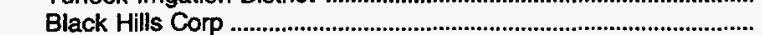 & Investor-Owned & 3 & 4 & 6 & 8 \\
\hline 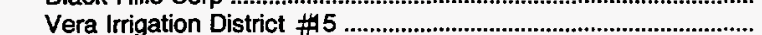 & Publicly Owned & 1 & 1 & 0 & 0 \\
\hline Vernon City of & Publicly Owned & 24 & 23 & 22 & 23 \\
\hline Washington Water Power Co & Investor-Owned & 234 & 391 & 488 & 634 \\
\hline Yellowstone Vlly Elec Coop Inc & Cooperative & 4 & 5 & 6 & 11 \\
\hline 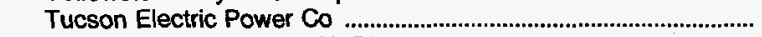 & Investor-Owned & 19 & 47 & 72 & 167 \\
\hline 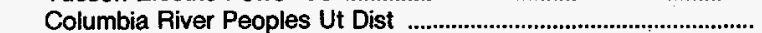 & Publicly Owned & 1 & - & - & - \\
\hline Northern California Power Agny ...................................................... & Publicly Owned & 29 & - & - & - \\
\hline 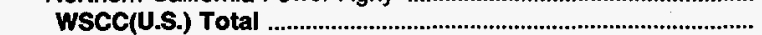 & & 14,491 & 17,954 & 20,364 & 32,759 \\
\hline Contiguous U.S. & & 35,554 & 45,275 & $\mathbf{5 2 , 6 3 2}$ & 88,673 \\
\hline \multicolumn{6}{|l|}{ Ascc } \\
\hline Alaska Electric Light\&Power Co & Investor-Owned & * & * & * & * \\
\hline 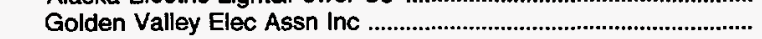 & Cooperative & * & 2 & 5 & 15 \\
\hline ASCC Total & & $\bullet$ & 2 & 5 & 15 \\
\hline
\end{tabular}

See footnotes at end of table. 
Table 9. U.S. Electric Utility Energy Savings by North American Electric Reliability Council Region and Hawaii by Class of Ownership, 1992, 1993, 1994 and 1998

(Million Kilowatthours) (Continued)

\begin{tabular}{|c|c|c|c|c|c|}
\hline \multirow{2}{*}{$\begin{array}{l}\text { North American Electric Reliability } \\
\text { Council Region and Hawail / } \\
\text { Electric Utility }\end{array}$} & \multirow{2}{*}{$\begin{array}{l}\text { Class of } \\
\text { Ownership }\end{array}$} & \multicolumn{2}{|c|}{ Historical Savings } & \multicolumn{2}{|c|}{ Prolected Savings } \\
\hline & & 1992 & 1993 & 1994 & 1998 \\
\hline $\begin{array}{l}\text { Hawail } \\
\text { Hawaii Electric Light Co Inc } \\
\text { Maui Electric Co Ltd } \\
\text { Hawaiian Electric Co Inc } \\
\text { Hawail Total }\end{array}$ & $\begin{array}{l}\text { Investor-Owned } \\
\text { Investor-Owned } \\
\text { Investor-Owned }\end{array}$ & $\begin{array}{r}- \\
5 \\
4 \\
9 \\
35,563\end{array}$ & $\begin{array}{r}2 \\
5 \\
10 \\
17 \\
45,294\end{array}$ & $\begin{array}{r}5 \\
3 \\
11 \\
18 \\
52,655\end{array}$ & $\begin{array}{r}57 \\
47 \\
186 \\
290 \\
88,978\end{array}$ \\
\hline
\end{tabular}

- Value less than 0.5

Notes: -Data are final. -Data are provided for electric utilities with sales to ultimate consumers or sales for resale greater than or equal to 120,000 megawatthours. $*$ Totals may not equal sum of components because of independent rounding.

Source: Energy Information Administration, Form ElA-861, “Annual Electric Utility Report." 
Table 10. U.S. Electric Utility Energy Savings by North American Electric Reliability Council Region and Hawaii by DSM Program Category, 1993

(Million Kilowatthours)

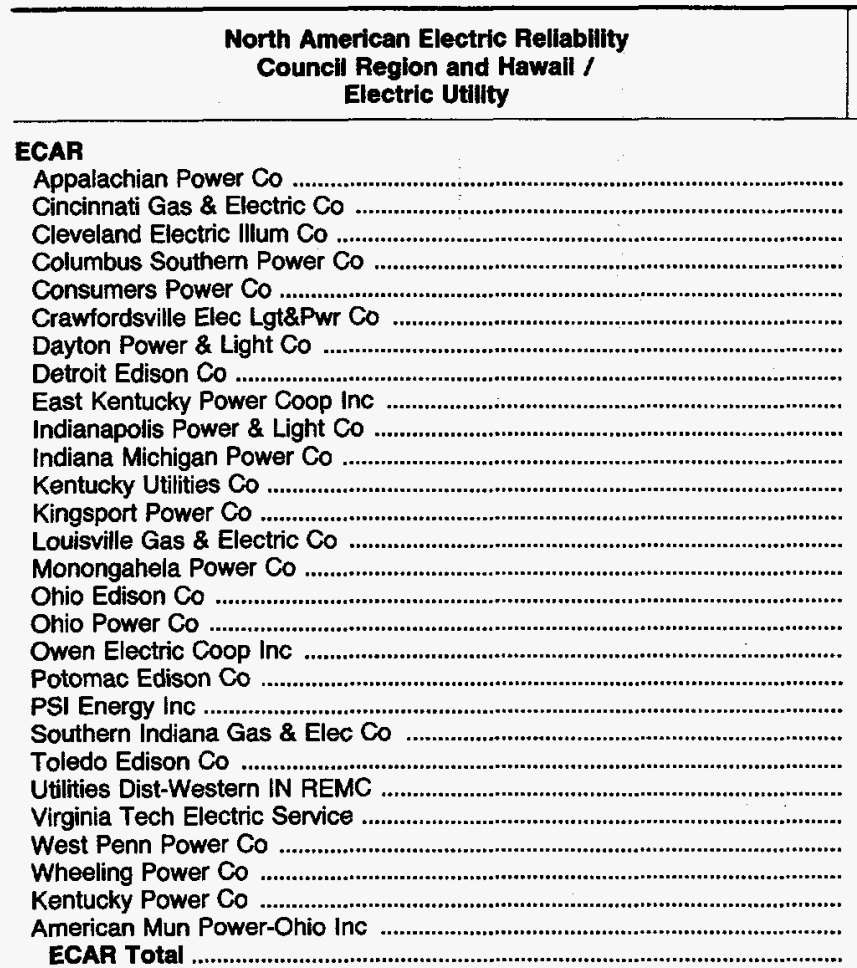

Energy
Efficiency

Load
Management

Total DSM

Programs

Appalachian Power Co

Crawfordsville Elec Lgt\&Pwr Co

Power \& Light Co

East Kentucky Power Coop Inc

Kentucky Utilities Co

Kingsport Power Co

Onio Edison $\mathrm{C}$

Owen Electric Coop Inc

Potomac Edison Co

Southern Indiana

est Penn Power Co

Kentucky Power Co

ECAR Total

$\begin{array}{rrr}448 & * & 448 \\ 6 & 0 & 6 \\ 8 & * & 8 \\ 130 & 56 & 185 \\ 1 & 0 & 1 \\ 0 & * & 130 \\ 133 & -3 & 4 \\ 4 & 0 & 105 \\ 105 & 0 & 2 \\ 2 & 0 & * \\ * & 0 & 1 \\ 1 & 0 & 62 \\ 21 & 41 & 11 \\ 11 & * & 105 \\ 39 & 66 & 1,221 \\ 1,221 & 0 & 2,288 \\ 2,128 & 160 & \end{array}$

ERCOT

Austin City of

Brazos Electric Power Coop Ine

Bryan City of

Central Power \& Light Co

Georgetown City of

Greenville Electric Util Sys

Houston Lighting \& Power Co

Johnson County Elec Coop Assn

Lower Colorado River Authority

Magic Valley Electric Coop Inc

San Bernard Electric Coop Inc

Tri-County Electric Coop

West Texas Utilities Co

San Marcos City of

Texas-New Mexico Power Co

Texas Utilities Electric $\mathrm{Co}$

ERCOT Total

\section{MAAC}

A \& N Electric Coop

Atlantic City Eler

Baltimore Gas \& Electric Co

Conowingo Power Co

Delmarva Power \& Light Co

Easton Utilities Comm

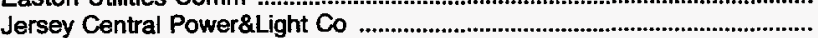

Metropolitan Edison Co

Pennsylvania Electric Co

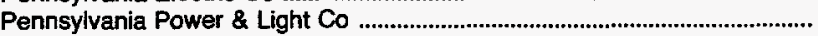

Philadelphia Electric Co

Potomac Electric Power Co

Public Service Electric\&Gas Co

Southern Maryland El Coop Inc

UGI Utilities Inc

See footnotes at end of table. 
Table 10. U.S. Electric Utility Energy Savings by North American Electric Reliability Council Region and Hawail by DSM Program Category, 1993

(Million Kilowatthours) (Continued)

\begin{tabular}{|c|c|c|c|}
\hline $\begin{array}{c}\text { North American Electric Reliablilty } \\
\text { Council Reglon and Hawall / } \\
\text { Electric Utlity }\end{array}$ & $\begin{array}{c}\text { Energy } \\
\text { Efflciency }\end{array}$ & $\begin{array}{c}\text { Load } \\
\text { Management }\end{array}$ & $\begin{array}{l}\text { Total DSM } \\
\text { Programs }\end{array}$ \\
\hline
\end{tabular}

MAAC

Adams Electric Coop Inc

Bedford Rural Elec Coop Inc

MAAC Total

MAIN

Central Illinois Light Co

Coles-Moultrie Electric CoOP

Columbia City of

Commonwealth Edison Co

Eastern Illini Electric Coop

Illinois Power Co

Madison Gas \& Electric Co

Manitowoc Public Utilities

Marshfield City of

Southeastern IL Elec Coop Inc

Southwestern Electric Coop Inc

Springfield City of

Wisconsin Electric Power $\mathrm{Co}$

Wisconsin Power \& Light Co

Wisconsin Public Power Inc Sys

Wisconsin Public Service Corp

MAIN Total

MAPP(U.S.)

Ames City of

Austin City of

Barron Electric Coop

Beatrice City of

Cedar Falls City of

Central lowa Power Coop

Central Power Elec Coop inc

Clark Electric Coop

Coop Power Assn

Cornhusker Public Power Dist

Fairmont Public Utilities Comm

Freeborn-Mower Electric Coop

Grant-Lafayette Electric Coop

IES Utilities Inc

Interstate Power Co

lowa Lakes Electric Coop

Lincoln Electric System

Marshall City of

Nodak Electric Coop inc

Midland Power Coop

Minnesota Power \& Light Co

Minnkota Power Coop inc

Moorhead City of

Muscatine City of

North Platte City of

Northern States Power Co

Northern States Power Co

Northwest lowa Power Coop

Oakdale Electric Coop

Omaha Public Power District

Otter Tail Power Co

Owatonna City of

People's Coop Power Assn

Pella City of

Rochester Public Utilities

Runestone Electric Assn

Shakopee Public Utilities Comm

Superior Water Light\&Power Co

Tri-County Electric Coop

United Power Assn

Verendrye Electric Coop Inc

Vernon Electric Coop

See footnotes at end of table.
1
0
944

$\begin{array}{rr}0 & 1 \\ * & \\ 206 & 1,150\end{array}$

$\begin{array}{rrr}0 & * & * \\ 0 & * & * \\ 4 & 0 & 4 \\ 1 & 1 & 2 \\ 1 & 2 & 3 \\ 34 & 0 & 34 \\ 90 & 0 & 90 \\ 9 & .0 & 9 \\ 2 & 0 & 2 \\ 0 & 124 & 124 \\ 1 & 28 & 29 \\ 6 & 0 & 6 \\ 1,272 & 14 & 1,286 \\ 197 & 0 & 197 \\ 16 & 0 & 16 \\ 322 & 1 & 322 \\ 1,955 & 170 & 2,125\end{array}$

\begin{tabular}{|c|c|c|}
\hline 1 & 0 & 1 \\
\hline * & 0 & . \\
\hline 0 & " & * \\
\hline 19 & 0 & 19 \\
\hline 7 & 41 & 48 \\
\hline 0 & $\because$ & * \\
\hline & 0 & * \\
\hline 10 & 0 & 10 \\
\hline 0 & * & * \\
\hline * & 1 & 1 \\
\hline * & * & 1 \\
\hline 29 & -9 & 21 \\
\hline 34 & 0 & 34 \\
\hline 4 & 33 & 38 \\
\hline 14 & 0 & 14 \\
\hline 0 & * & * \\
\hline 0 & 2 & 2 \\
\hline & 6 & 6 \\
\hline 27 & 0 & 27 \\
\hline 0 & 7 & 7 \\
\hline 2 & 0 & 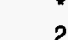 \\
\hline 0 & . & * \\
\hline 41 & 45 & 86 \\
\hline 996 & 13 & 1,009 \\
\hline 6 & 0 & 6 \\
\hline 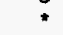 & 0 & * \\
\hline 1 & 0 & 1 \\
\hline 18 & 20 & 38 \\
\hline 0 & 1 & 1 \\
\hline 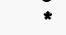 & 0 & * \\
\hline 2 & 0 & 2 \\
\hline 1 & * & 2 \\
\hline 0 & * & * \\
\hline 2 & * & 2 \\
\hline & 0 & * \\
\hline 7 & 17 & 23 \\
\hline & 0 & * \\
\hline t & 0 & * \\
\hline
\end{tabular}


Table 10. U.S. Electric Utility Energy Savings by North American Electric Reliability Council Region and Hawaii by DSM Program Category, 1993

(Million Kilowatthours) (Continued)

\begin{tabular}{|c|c|c|c|}
\hline $\begin{array}{c}\text { North American Electric Reliability } \\
\text { Council Region and Hawail / } \\
\text { Electric Utllty }\end{array}$ & $\begin{array}{l}\text { Energy } \\
\text { Efficiency }\end{array}$ & $\begin{array}{c}\text { Load } \\
\text { Management }\end{array}$ & $\begin{array}{l}\text { Total DSM } \\
\text { Programs }\end{array}$ \\
\hline
\end{tabular}

MAPP(U.S.)

Mountrail-Williams El Coop Inc

Wild Rice Electric Coop Inc

Municipal Energy Agency of NE

Midwest Power Systems Inc .

Cass County Electric Coop Inc

MAPP(U.S.) Total

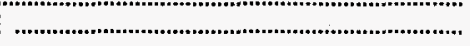



NPCC(U.S.)

Bangor Hydro-Electric Co

Blackstone Valley Electric Co

Boston Edison Co

Braintree Town of

Burlington City of

Cambridge Electric Light $\mathrm{CO}$

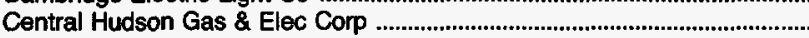

Central Maine Power Co

Central Vermont Pub Serv Corp

Chicopee City of

Citizens Utilities Co

Commonwealth Electric Co

Concord Electric Co

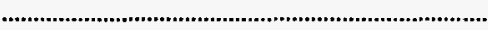

Connecticut Light \& Power Co

Connecticut Valley Elec Co Inc

Eastern Edison Co

Exeter \& Hampton Electric Co

Fitchburg Gas \& Elec Light Co

Green Mountain Power Corp

Holyoke City of

Hingham City of

Jamestown City of

Littleton Town of

Long Island Lighting $\mathrm{Co}_{0}$

Maine Public Service Co

Massena Town of

Montaup Electric Co

Narragansett Electric Co

New Hampshire Elec Coop Inc.....

Now York State Elec \& Gas Corp

Newport Electric Corp

Niagara Mohawk Power Corp

Norwood City of

Orange \& Rockland Utils Inc

Power Authority of State of NY

Public Service Co of NH

Reading Town of

Shrews

Shrewsbury Town of

Taunton City of ...

United Illuminating $\mathrm{Co}_{0}$

Omya Inc

Granite State Electric Co

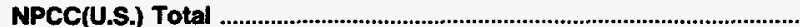

SERC

Aiken Electric Coop Inc

Alabama Electric Coop Inc

Alabama Power Co

Albemarle City of

Altamaha Electric Member Corp

Amicalola Electric Member Corp

BARC Electric Coop Inc .

Berkeley Electric Coop inc

Black River Electric Coop Inc

Canoochee Electric Member Corp

Carolina Power \& Light Co

$\begin{array}{rrr}1 & 13 & 14 \\ 0 & 56 & 56 \\ 1 & * & 1 \\ 104 & 2 & 105 \\ 1,329 & 2 & 2 \\ & \mathbf{2 5 2} & 1,581\end{array}$

38

382

382

9

71

97
390

31
4

4
2

120

1
1,159

2
496

94

8

30

-

See footnotes at end of table.

$\begin{array}{rrr}0 & 1 & 1 \\ 21 & 2 & 24 \\ 468 & -19 & 449 \\ 0 & \vdots & \vdots \\ \vdots & \vdots & \vdots \\ 0 & \vdots & 4 \\ 4 & 0 & 2 \\ 2 & \vdots & 2,062 \\ 0 & 0 & \end{array}$


Table 10. U.S. Electric Utility Energy Savings by North American Electric Reliability Council Region and Hawaii by DSM Program Category, 1993

(Million Kilowatthours) (Continued)

\begin{tabular}{|c|c|c|c|}
\hline $\begin{array}{l}\text { North American Electric Reliability } \\
\text { Council Region and Hawail / } \\
\text { Electric Utility }\end{array}$ & $\begin{array}{l}\text { Energy } \\
\text { Efficiency }\end{array}$ & $\begin{array}{c}\text { Load } \\
\text { Management }\end{array}$ & $\begin{array}{c}\text { Total DSA } \\
\text { Programs }\end{array}$ \\
\hline
\end{tabular}

SERC

Carroll Electric Member Corp

Central Georgia El Member Corp

.

Coast Electric Member Corp

Coastal Electric Member Corp

Cobb Electric Membership Corp

Community Electric Coop

Coweta-Fayette EI Member Corp

Crescent Electric Member Corp

Douglas City of

Excelsior Electric Member Corp

Fairfield Electric Coop inc

Fayetteville Public Works Comm

Fitzgerald Wtr Lgt \& Bond Comm

Flint Electric Membership Corp

Florida Power \& Light Co

Florida Power Corp

Fort Pierce Utilities Auth

Four County Elec Member Corp

Gainesville Regional Utilities

Georgia Power Co

Grady County Elec Member Corp

Greenville Utilities Comm

Gulf Power Co

Haywood Electric Member Corp

Jackson Electric Member Corp

Jacksonville Electric Auth

Jefferson Electric Member Corp

Kissimmee Utility Authority

Lakeland City of

Laurens Electric Coop Inc

Laurinburg City of ...

Lawrenceville City of

Lee County Electric Coop Inc

Leesburg City of

Lumbee River Elec Member Corp

Lumberton City of

Lynches River Elec Coop Inc

Manassas City of

Marietta City of

Mecklenburg Electric Coop Inc

Mid-Carolina Electric Coop inc

Mississippi Power Co

Monroe City of .

New Bern City of

Northern Virginia Elec Coop

Northern Neck Elec Coop Inc

Ocala City of

Orangeburg City of

Palmetto Electric Coop Inc

Orlando Utilities Comm

Randolph Electric Member Corp

Rayle Electric Membership Corp

Rock Hill City of

Satilla Rural Elec Member Corp

Savannah Electric \& Power Co

Sawnee Electric Members Corp

Shenandoah Valley Elec Coop

Singing River Elec Power Assn

South Carolina Electric8

South Carolina Pub Serv Auth

South Mississippi El Pwr Assn

Sumter Electric Coop Inc

\begin{tabular}{|c|c|c|}
\hline & & \\
\hline 2 & $\begin{array}{l}1 \\
0\end{array}$ & $\begin{array}{l}1 \\
2\end{array}$ \\
\hline$\star$ & * & 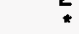 \\
\hline 0 & * & * \\
\hline 1 & 0 & 1 \\
\hline 16 & 0 & 16 \\
\hline 0 & * & 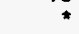 \\
\hline 56 & 1 & 57 \\
\hline 0 & 1 & 1 \\
\hline * & 1 & 1 \\
\hline 74 & 0 & 74 \\
\hline . & 0 & * \\
\hline : & " & * \\
\hline${ }_{0}^{*}$ & * & * \\
\hline * & 4 & 4 \\
\hline 0 & $*$ & * \\
\hline 2,726 & 13 & 2,738 \\
\hline 510 & 479 & 989 \\
\hline 1 & 0 & 1 \\
\hline 1 & 0 & $i$ \\
\hline 43 & 22 & 65 \\
\hline 134 & 0 & 134 \\
\hline 14 & 0 & 14 \\
\hline 418 & 0 & 418 \\
\hline 10 & 1 & 11 \\
\hline 108 & 0 & 108 \\
\hline & * & 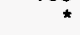 \\
\hline 0 & 2 & 2 \\
\hline 2 & 1 & 4 \\
\hline 1 & * & 1 \\
\hline 0 & * & 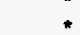 \\
\hline 0 & * & * \\
\hline 21 & 0 & 21 \\
\hline 0 & * & * \\
\hline 0 & 2 & 2 \\
\hline 0 & * & * \\
\hline 0 & 1 & 1 \\
\hline 0 & 3 & 3 \\
\hline 0 & 2 & 2 \\
\hline 0 & 6 & 6 \\
\hline 0 & 2 & 2 \\
\hline 1 & 0 & 1 \\
\hline 0 & * & : \\
\hline 0 & $*$ & * \\
\hline 0 & * & " \\
\hline * & * & 1 \\
\hline 0 & * & * \\
\hline 9 & * & 9 \\
\hline 0 & * & * \\
\hline 1 & * & 1 \\
\hline 74 & 0 & 74 \\
\hline 0 & * & : \\
\hline * & 0 & * \\
\hline 4 & 1 & 5 \\
\hline 0 & 1 & 1 \\
\hline * & * & * \\
\hline 1 & 0 & 1 \\
\hline 1 & 0 & 1 \\
\hline 0 & 1 & 1 \\
\hline 2 & * & 2 \\
\hline $15 \overline{3}$ & 26 & 179 \\
\hline 24 & 0 & 24 \\
\hline 13 & 0 & 13 \\
\hline 17 & * & 17 \\
\hline
\end{tabular}

See footnotes at end of table. 
Table 10. U.S. Electric Utility Energy Savings by North American Electric Reliability Council Region and Hawaii by DSM Program Category, 1993

(Million Kilowatthours) (Continued)

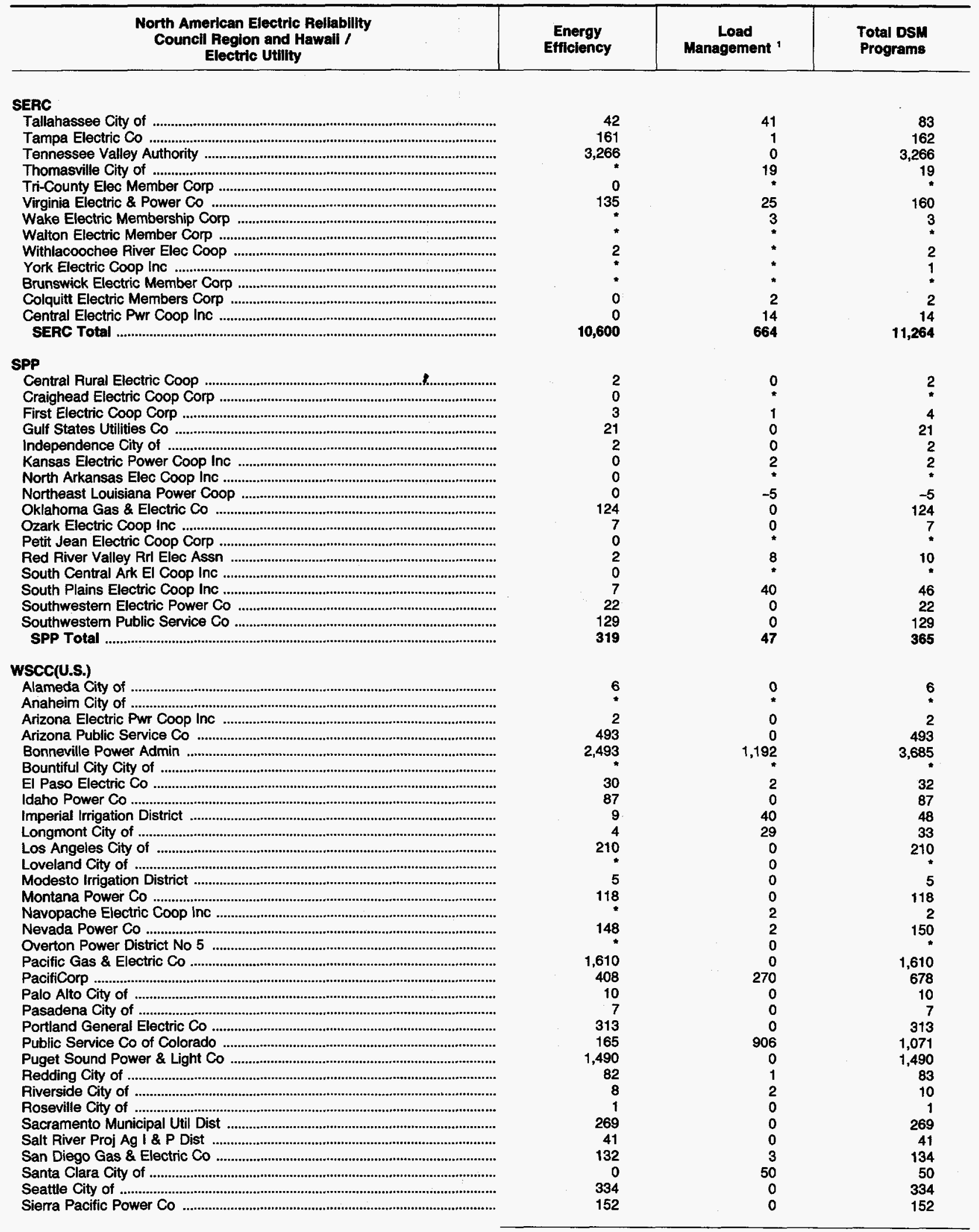

See footnotes at end of table. 
Table 10. U.S. Electric Utility Energy Savings by North American Electric Reliability Council Region and Hawaii by DSM Program Category, 1993

(Million Kilowatthours) (Continued)

\begin{tabular}{|c|c|c|c|}
\hline $\begin{array}{l}\text { North Ameriean Electric Rellability } \\
\text { Councll Reglon and Hawail / } \\
\text { Electric Utility }\end{array}$ & $\begin{array}{c}\text { Energy } \\
\text { Efficiency }\end{array}$ & $\begin{array}{c}\text { Load } \\
\text { Management ' }\end{array}$ & $\begin{array}{l}\text { Total DSM } \\
\text { Programs }\end{array}$ \\
\hline 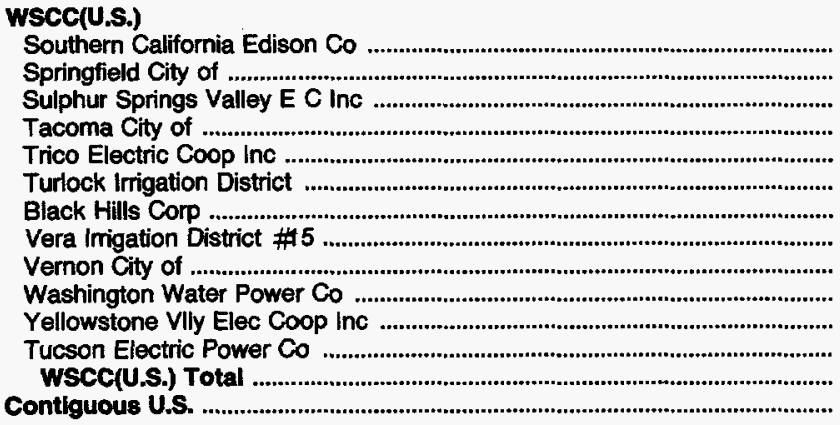 & $\begin{array}{r}6,113 \\
54 \\
* \\
183 \\
0 \\
5 \\
4 \\
\star \\
0 \\
391 \\
0 \\
47 \\
15,425 \\
41,100\end{array}$ & $\begin{array}{r}0 \\
* \\
* \\
0 \\
* \\
0 \\
0 \\
1 \\
23 \\
0 \\
5 \\
0 \\
2,528 \\
4,175\end{array}$ & $\begin{array}{r}6,113 \\
54 \\
\\
183 \\
\vdots \\
5 \\
4 \\
1 \\
23 \\
391 \\
5 \\
47 \\
17,954 \\
45,275\end{array}$ \\
\hline 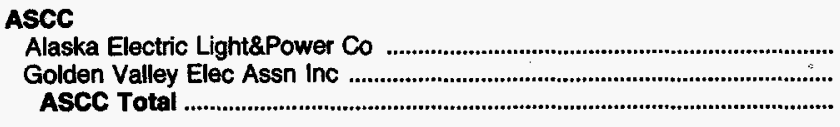 & $\begin{array}{l}0 \\
2 \\
2\end{array}$ & $\stackrel{*}{0}$ & $\begin{array}{l}* \\
2 \\
2\end{array}$ \\
\hline 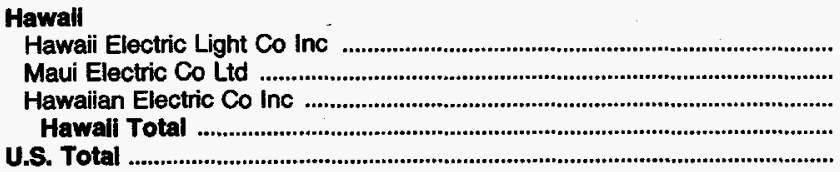 & $\begin{array}{r}2 \\
5 \\
10 \\
17 \\
41,119\end{array}$ & $\begin{array}{r}0 \\
0 \\
0 \\
0 \\
4,175\end{array}$ & $\begin{array}{r}2 \\
5 \\
10 \\
17 \\
45,294\end{array}$ \\
\hline
\end{tabular}

1 Load management includes the following DSM program categories: direct load control, interruptible load, other load management, other demand-side management.

- Value less than 0.5 .

Notes: Data are final. -Data are provided for electric utilities with sales to ultimate consumers or sales for resale greater than or equal to 120,000 megawatthours. - Totals may not equal sum of components because of independent rounding.

Source: Energy Information Administration, Form ElA-861, "Annual Electric Utility Report." 
Table 11. U.S. Electric Utility Energy Savings by North American Electric Reliability Council Region and Hawaii by Class of Ownership and Sector, 1993 (Million Kilowatthours)

\begin{tabular}{|c|c|c|c|c|c|c|}
\hline $\begin{array}{c}\text { North American Electric Reliabillty } \\
\text { Council Region and Hawail } / \\
\text { Electric Utllity }\end{array}$ & $\begin{array}{l}\text { Class of } \\
\text { Ownership }\end{array}$ & Residential & Commercial & Industrial & Other & Total \\
\hline 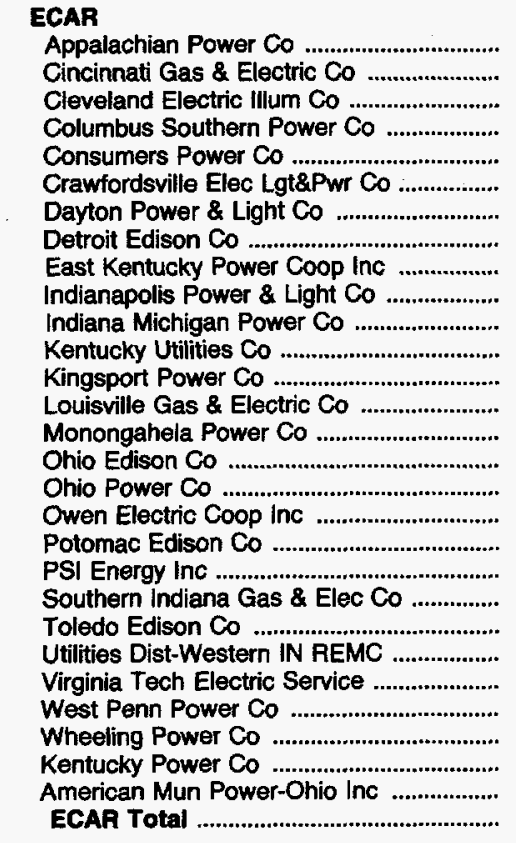 & $\begin{array}{l}\text { Investor-Owned } \\
\text { Investor-Owned } \\
\text { Investor-Owned } \\
\text { Investor-Owned } \\
\text { Investor-Owned } \\
\text { Publicly Owned } \\
\text { Investor-Owned } \\
\text { Investor-Owned } \\
\text { Cooperative } \\
\text { Investor-Owned } \\
\text { Investor-Owned } \\
\text { Investor-Owned } \\
\text { Investor-Owned } \\
\text { Investor-Owned } \\
\text { Investor-Owned } \\
\text { Investor-Owned } \\
\text { Investor-Owned } \\
\text { Cooperative } \\
\text { Investor-Owned } \\
\text { Investor-Owned } \\
\text { Investor-Owned } \\
\text { Investor-Owned } \\
\text { Cooperative } \\
\text { Publicly Owned } \\
\text { Investor-Owned } \\
\text { Investor-Owned } \\
\text { Investor-Owned } \\
\text { Publicly Owned }\end{array}$ & 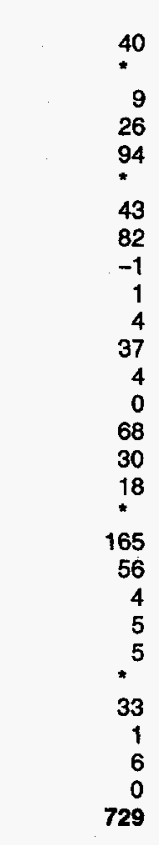 & $\begin{array}{r}0 \\
6 \\
4 \\
0 \\
93 \\
0 \\
20 \\
34 \\
0 \\
12 \\
0 \\
0 \\
0 \\
0 \\
60 \\
13 \\
0 \\
* \\
78 \\
46 \\
4 \\
7 \\
2 \\
* \\
82 \\
0 \\
0 \\
0 \\
462\end{array}$ & $\begin{array}{r}* \\
* \\
* \\
93 \\
0 \\
27 \\
32 \\
0 \\
28 \\
* \\
0 \\
0 \\
16 \\
89 \\
18 \\
* \\
* \\
71 \\
40 \\
9 \\
4 \\
4 \\
* \\
148 \\
0 \\
* \\
* \\
587\end{array}$ & $\begin{array}{l}0 \\
0 \\
0 \\
0 \\
0 \\
* \\
0 \\
0 \\
0 \\
0 \\
0 \\
0 \\
0 \\
0 \\
0 \\
0 \\
0 \\
0 \\
0 \\
0 \\
0 \\
0 \\
0 \\
* \\
0 \\
0 \\
0 \\
* \\
*\end{array}$ & $\begin{array}{r}40 \\
6 \\
19 \\
26 \\
280 \\
* \\
90 \\
149 \\
-1 \\
41 \\
4 \\
37 \\
4 \\
16 \\
217 \\
61 \\
18 \\
1 \\
314 \\
141 \\
16 \\
16 \\
12 \\
* \\
263 \\
1 \\
6 \\
* \\
1,779\end{array}$ \\
\hline  & $\begin{array}{l}\text { Publicly Owned } \\
\text { Cooperative } \\
\text { Publicly Owned } \\
\text { Investor-Owned } \\
\text { Publicly Owned } \\
\text { Publicly Owned } \\
\text { Investor-Owned } \\
\text { Cooperative } \\
\text { Publicly Owned } \\
\text { Cooperative } \\
\text { Cooperative } \\
\text { Cooperative } \\
\text { Investor-Owned } \\
\text { Publicly Owned } \\
\text { Investor-Owned } \\
\text { Investor-Owned }\end{array}$ & $\begin{array}{r}229 \\
6 \\
8 \\
71 \\
1 \\
0 \\
43 \\
4 \\
97 \\
1 \\
* \\
1 \\
22 \\
9 \\
38 \\
983 \\
1,511\end{array}$ & $\begin{array}{r}219 \\
0 \\
* \\
115 \\
* \\
0 \\
79 \\
0 \\
8 \\
0 \\
0 \\
0 \\
3 \\
2 \\
1 \\
238 \\
665\end{array}$ & $\begin{array}{r}0 \\
0 \\
0 \\
0 \\
0 \\
* \\
8 \\
0 \\
0 \\
0 \\
0 \\
0 \\
37 \\
0 \\
66 \\
0 \\
111\end{array}$ & $\begin{array}{l}0 \\
0 \\
0 \\
0 \\
0 \\
0 \\
0 \\
0 \\
0 \\
1 \\
0 \\
0 \\
0 \\
0 \\
0 \\
0 \\
1\end{array}$ & $\begin{array}{r}448 \\
6 \\
8 \\
185 \\
1 \\
* \\
130 \\
4 \\
105 \\
2 \\
* \\
1 \\
62 \\
11 \\
105 \\
1,221 \\
2,288\end{array}$ \\
\hline 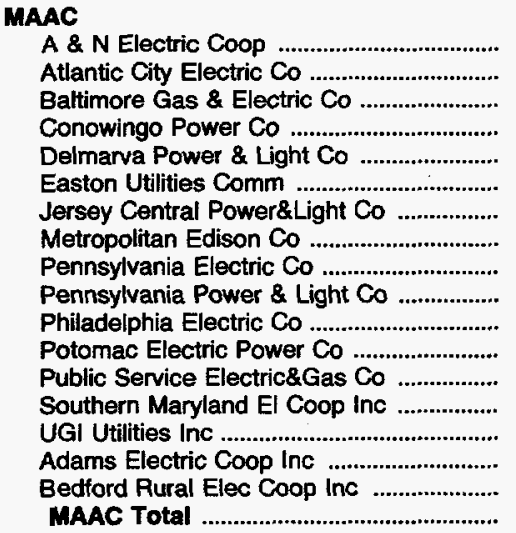 & $\begin{array}{l}\text { Cooperative } \\
\text { Investor-Owned } \\
\text { Investor-Owned } \\
\text { Investor-Owned } \\
\text { Investor-Owned } \\
\text { Publicly Owned } \\
\text { Investor-Owned } \\
\text { Investor-Owned } \\
\text { Investor-Owned } \\
\text { Investor-Owned } \\
\text { Investor-Owned } \\
\text { Investor-Owned } \\
\text { Investor-Owned } \\
\text { Cooperative } \\
\text { Investor-Owned } \\
\text { Cooperative } \\
\text { Cooperative }\end{array}$ & $\begin{array}{r}1 \\
43 \\
23 \\
4 \\
3 \\
* \\
38 \\
62 \\
23 \\
24 \\
60 \\
33 \\
3 \\
4 \\
* \\
0 \\
* \\
321\end{array}$ & $\begin{array}{r}0 \\
15 \\
165 \\
0 \\
43 \\
0 \\
34 \\
5 \\
23 \\
4 \\
* \\
398 \\
43 \\
0 \\
0 \\
0 \\
0 \\
730\end{array}$ & $\begin{array}{r}0 \\
1 \\
2 \\
0 \\
0 \\
0 \\
34 \\
14 \\
30 \\
1 \\
0 \\
0 \\
10 \\
0 \\
0 \\
0 \\
0 \\
93\end{array}$ & $\begin{array}{l}0 \\
6 \\
0 \\
0 \\
0 \\
0 \\
0 \\
0 \\
0 \\
0 \\
0 \\
0 \\
0 \\
0 \\
0 \\
1 \\
0 \\
7\end{array}$ & $\begin{array}{r}1 \\
65 \\
190 \\
4 \\
46 \\
* \\
106 \\
81 \\
75 \\
29 \\
60 \\
431 \\
57 \\
4 \\
* \\
1 \\
* 1,150\end{array}$ \\
\hline
\end{tabular}

See footnotes at end of table. 
Table 11. U.S. Electric Utility Energy Savings by North American Electric Reliability Council Region and Hawaii by Class of Ownership and Sector, 1993 (Million Kilowatthours) (Continued)

\begin{tabular}{c|c|c|c|c|c|c|c|}
\hline $\begin{array}{c}\text { North American Electric Reliability } \\
\text { Council Reglon and Hawall / } \\
\text { Electric Utility }\end{array}$ & $\begin{array}{c}\text { Class of } \\
\text { Ownership }\end{array}$ & Residential & Commercial & Industrial & Other \\
\hline
\end{tabular}

MAIN

Central Illinois Light Co

Coles-Moultrie Electric Coop

Columbia City of

Common

Eastern Illini Electric Coop

IIlinois Power $\mathrm{Co}$

Madison Gas \& Electric Co

Manitowoc Public Utilities

Marshfield City of

Southeastern IL Elec Coop Inc

Southwestern Electric CoOp Inc

Springfield City of

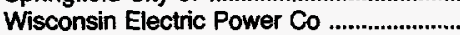

Wisconsin Power \& Light Co

Wisconsin Public Power inc Sys

Wisconsin Public Service Corp

MAIN Total

MAPP(U.S.)

Ames City of

Austin City of

Barron Electr

Beatrice City of

Cedar Falls City of

Central Jowa Power Coop

Central Power Elec Coop Inc

Clark Electric Coop

Coop Power Assn

Cornhusker Public Power Dist

Fairmont Public Utilities Comm

Freeborn-Mower Electric Coop

Grant-Lafayette Electric Coop

IES Utilities Inc

Interstate Power Co

lowa Lakes Electric Coop

Lincoln Electric System

Marshall City of

Nodak Electric Coop Inc

Midland Power Coop

Minnkota Power Coop Inc

Moorhead City of

Muscatine City of

North Platte City of

Northern States Power Co

Northern States Power Co

Northwest lowa Power Coop

Oakdale Electric Coop

ail Power Co

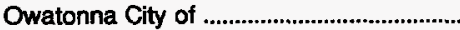

People's Coop Power Assn

Pella City of

Rochester Public Utilities

Runestone Electric Assn

Shakopee Public Utilities Comm

Superior Water Light\&Power Co ...............

Tri-County Electric Coop ...............................

United Power Assn

Verendrye Electric Coop inc

Vernon Electric Coop

Mountrail-Williams

Wild Rice Electric Coop Inc

Municipal Energy Agency of NE

Midwest Power Systems Inc ...

Cass County Electric Coop Inc

MAPP(U.S.) Total
Investor-Owned

Cooperative

Publicly Owned

Investor-Owned

Cooperative

Investor-Owned

Investor-Owned

Publicly Owned

Publicly Owned

Cooperative

Cooperative

Publicly Owned

Investor-Owned

investor-Owned

Publicly Owned

Investor-Owned

Publicly Owned

Publicly Owned

Cooperative

Publicly Owned

Publicly Owned

Cooperative

Cooperative

Cooperative

Cooperative

Publicly Owned

Publicly Owned

Cooperative

Cooperative

Investor-Owned

Investor-Owned

Cooperative

Publicly Owned

Publicly Owned

Cooperative

Cooperative

Investor-Owned

Cooperative

Publicly Owned

Publicly Owned

Publicly Owned

Investor-Owned

Investor-Owned

Cooperative

Cooperative

Publicly Owned

Investor-Owned

Publicly Owned

Cooperative

Publicly Owned

Publicly Owned

Cooperative

Publicly Owned

investor-Owned

Cooperative

Cooperative

Cooperative

Cooperative

Cooperative

Cooperative

Publicly Owned

Investor-Owned

Cooperative

0
2
1
3
0
14
3
124
23
383
19
100
680

0
0
2
1
0
33
63
3
2
0
5
3
617
167
5
206
1,108

0
$*$
0
$*$
1
0
3
1
0
1
0
285
12
6
0
308

2

34

2

124

29

286

197

16

322

100
680

1,108

2,125

$\begin{array}{lc}0 & 0 \\ * & * \\ * & * \\ 19 & * \\ 47 & 1 \\ 0 & 0 \\ * & 10\end{array}$

0

*

$-1$

2

$+$

*

1

2

7

1

0

24
118

118

See footnotes at end of table. 
Table 11. U.S. Electric Utility Energy Savings by North American Electric Reliability Council Region and Hawail by Class of Ownership and Sector, 1993 (Million Kilowatthours) (Continued)

\begin{tabular}{|c|c|c|c|c|c|c|}
\hline $\begin{array}{c}\text { North American Electric Reliability } \\
\text { Councll Region and Hawall / } \\
\text { Electric Utility }\end{array}$ & $\begin{array}{c}\text { Class of } \\
\text { Ownership }\end{array}$ & Residential & Commerclal & Industrial & Other & Total \\
\hline  & $\begin{array}{l}\text { Investor-Owned } \\
\text { Investor-Owned } \\
\text { Investor-Owned } \\
\text { Publicly Owned } \\
\text { Publicly Owned } \\
\text { Investor-Owned } \\
\text { Investor-Owned } \\
\text { Investor-Owned } \\
\text { Investor-Owned } \\
\text { Publicly Owned } \\
\text { Investor-Owned } \\
\text { Investor-Owned } \\
\text { Investor-Owned } \\
\text { Investor-Owned } \\
\text { Investor-Owned } \\
\text { Investor-Owned } \\
\text { Investor-Owned } \\
\text { Investor-Owned } \\
\text { Investor-Owned } \\
\text { Investor-Owned } \\
\text { Publicly Owned } \\
\text { Publicly Owned } \\
\text { Publicly Owned } \\
\text { Publicly Owned } \\
\text { Investor-Owned } \\
\text { Investor-Owned } \\
\text { Investor-Owned } \\
\text { Publicly Owned } \\
\text { Investor-Owned } \\
\text { Investor-Owned } \\
\text { Cooperative } \\
\text { Investor-Owned } \\
\text { Investor-Owned } \\
\text { Investor-Owned } \\
\text { Publicly Owned } \\
\text { Investor-Owned } \\
\text { Publicly Owned } \\
\text { Investor-Owned } \\
\text { Publicly Owned } \\
\text { Investor-Owned } \\
\text { Publicly Owned } \\
\text { Publicly Owned } \\
\text { Investor-Owned } \\
\text { Investor-Owned } \\
\text { Investor-Owned } \\
\text { Investor-Owned }\end{array}$ & 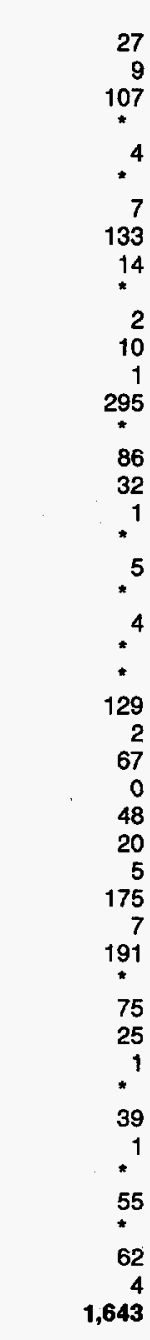 & $\begin{array}{r}8 \\
31 \\
206 \\
* \\
1 \\
18 \\
73 \\
201 \\
14 \\
3 \\
0 \\
111 \\
* \\
734 \\
1 \\
413 \\
55 \\
* \\
3 \\
25 \\
0 \\
* \\
* \\
0 \\
451 \\
2 \\
424 \\
0 \\
93 \\
142 \\
2 \\
519 \\
7 \\
467 \\
* \\
92 \\
56 \\
* 417 \\
* \\
57 \\
0 \\
16 \\
63 \\
0 \\
11 \\
\end{array}$ & 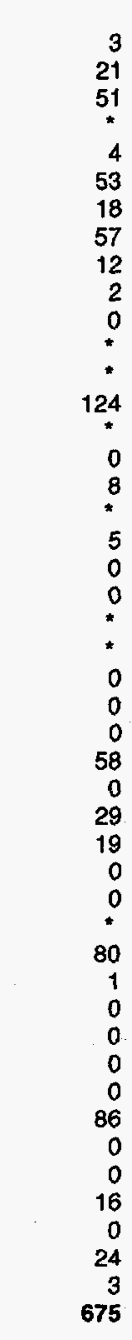 & $\begin{array}{r}0 \\
0 \\
17 \\
0 \\
0 \\
0 \\
0 \\
0 \\
0 \\
0 \\
0 \\
0 \\
0 \\
0 \\
0 \\
0 \\
0 \\
0 \\
0 \\
0 \\
0 \\
0 \\
0 \\
0 \\
0 \\
0 \\
0 \\
0 \\
0 \\
0 \\
0 \\
0 \\
0 \\
0\end{array}$ & $\begin{array}{r}38 \\
61 \\
382 \\
* \\
9 \\
71 \\
97 \\
390 \\
41 \\
4 \\
2 \\
121 \\
1 \\
1,160 \\
2 \\
498 \\
94 \\
1 \\
8 \\
30 \\
* \\
4 \\
* \\
* \\
580 \\
5 \\
549 \\
1 \\
170 \\
181 \\
7 \\
695 \\
15 \\
737 \\
1 \\
167 \\
81 \\
1 \\
* 769\end{array}$ \\
\hline 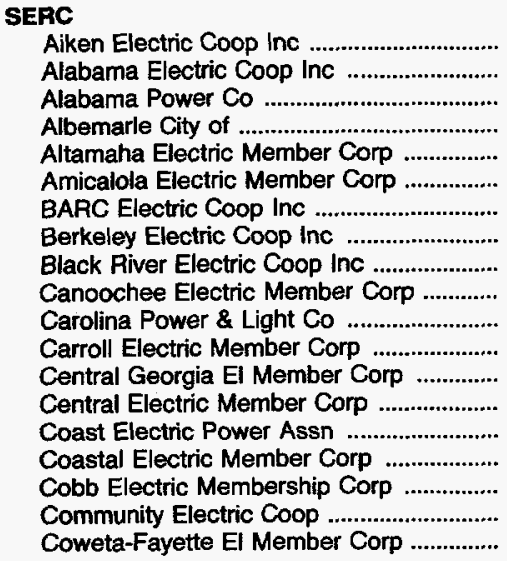 & $\begin{array}{l}\text { Cooperative } \\
\text { Cooperative } \\
\text { Investor-Owned } \\
\text { Publicly Owned } \\
\text { Cooperative } \\
\text { Cooperative } \\
\text { Cooperative } \\
\text { Cooperative } \\
\text { Cooperative } \\
\text { Cooperative } \\
\text { Investor-Owned } \\
\text { Cooperative } \\
\text { Cooperative } \\
\text { Cooperative } \\
\text { Cooperative } \\
\text { Cooperative } \\
\text { Cooperative } \\
\text { Cooperative } \\
\text { Cooperative }\end{array}$ & $\begin{array}{r}1 \\
24 \\
468 \\
0 \\
* \\
* \\
* \\
4 \\
2 \\
{ }^{*} \\
776 \\
* \\
2 \\
* \\
* \\
1 \\
16 \\
* \\
57\end{array}$ & $\begin{array}{r}0 \\
0 \\
-19 \\
0 \\
* \\
0 \\
0 \\
0 \\
0 \\
0 \\
392 \\
* \\
0 \\
0 \\
* \\
0 \\
0 \\
0 \\
0\end{array}$ & $\begin{array}{c}0 \\
0 \\
0 \\
* \\
0 \\
0 \\
0 \\
0 \\
0 \\
0 \\
894 \\
* \\
0 \\
* \\
0 \\
0 \\
0 \\
0 \\
0\end{array}$ & $\begin{array}{l}0 \\
0 \\
0 \\
0 \\
\vdots \\
0 \\
0 \\
0 \\
0 \\
* \\
0 \\
* \\
0 \\
0 \\
0 \\
0 \\
0 \\
0 \\
0\end{array}$ & $\begin{array}{r}24 \\
449 \\
* \\
* \\
4 \\
2 \\
2,062 \\
1 \\
2 \\
* \\
1 \\
16 \\
57\end{array}$ \\
\hline
\end{tabular}

See footnotes at end of table. 
Table 11. U.S. Electric Utility Energy Savings by North American Electric Reliability Council Region and Hawail by Class of Ownership and Sector, 1993 (Million Kilowatthours) (Continued)

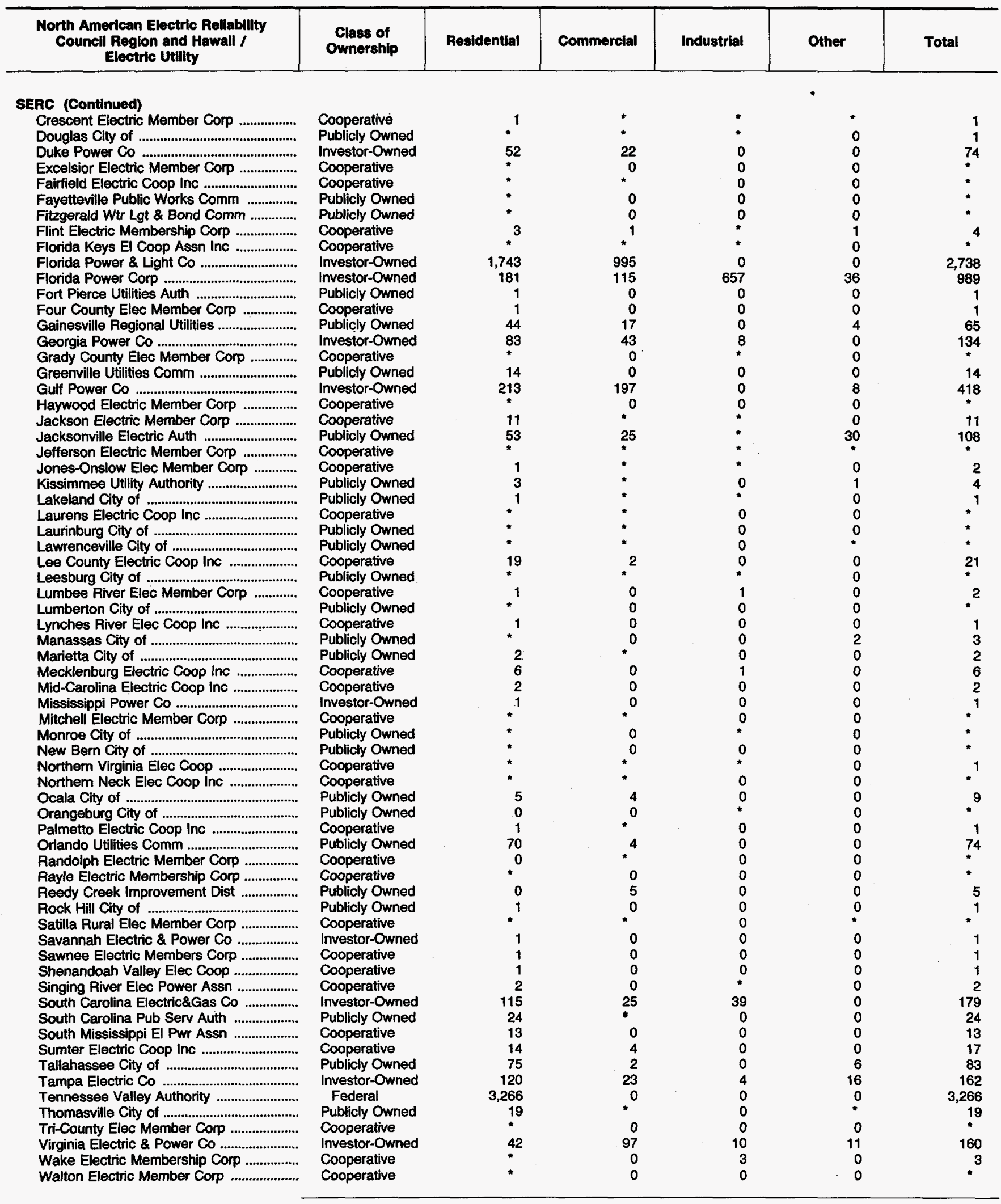

See footnotes at end of table. 
Table 11. U.S. Electric Utility Energy Savings by North American Electric Reliability Council Region and Hawail by Class of Ownership and Sector, 1993 (Million Kilowatthours) (Continued)

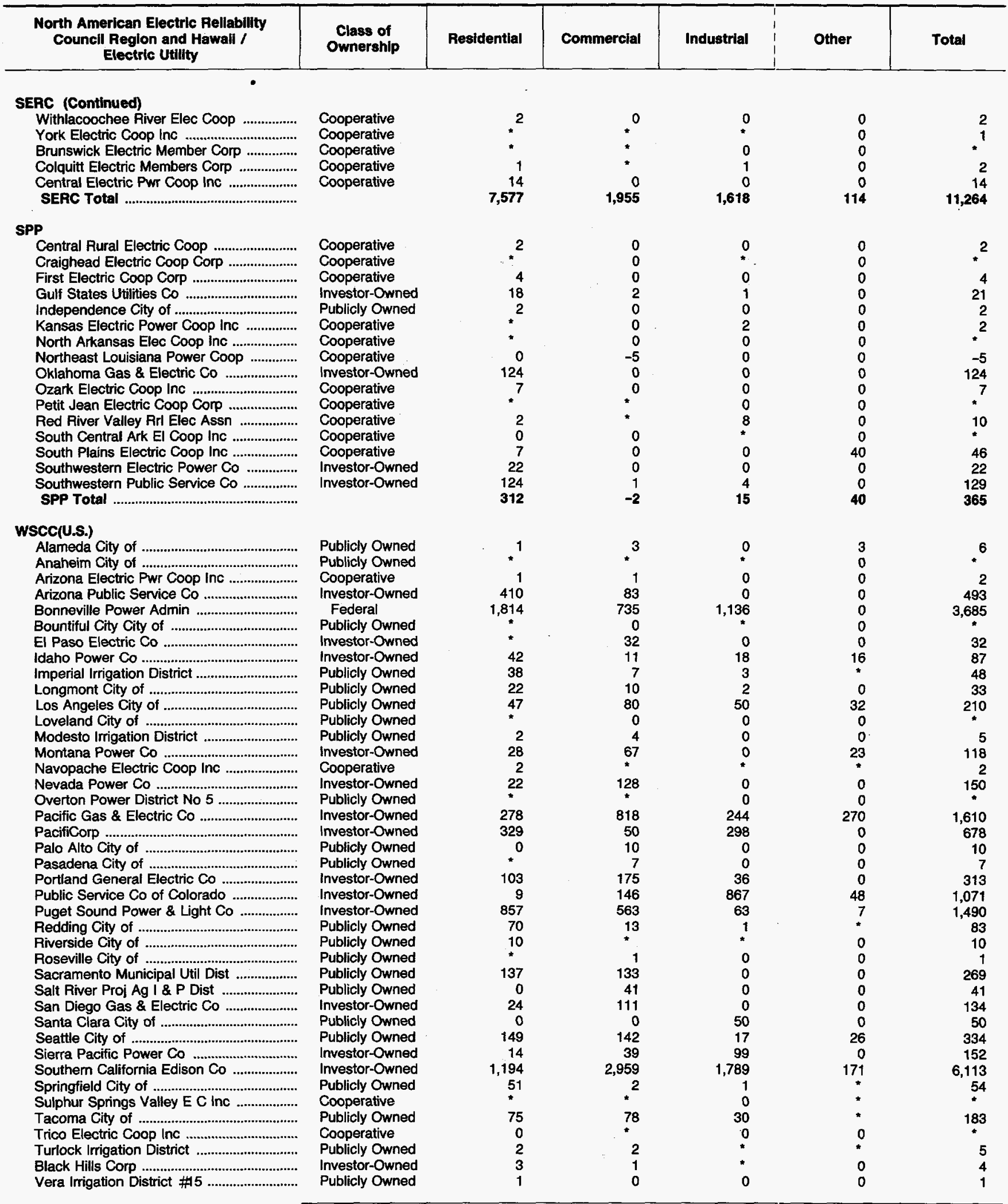

See footnotes at end of table. 
Table 11. U.S. Electric Utility Energy Savings by North American Electric Reliability Council Region and Hawaii by Class of Ownership and Sector, 1993

(Million Kilowatthours) (Continued)

\begin{tabular}{|c|c|c|c|c|c|c|}
\hline $\begin{array}{l}\text { North American Electric Reliability } \\
\text { Council Region and Hawail / } \\
\text { Electric Utility }\end{array}$ & $\begin{array}{l}\text { Class of } \\
\text { Ownership }\end{array}$ & Residential & Commercial & Industrial & Other & Total \\
\hline 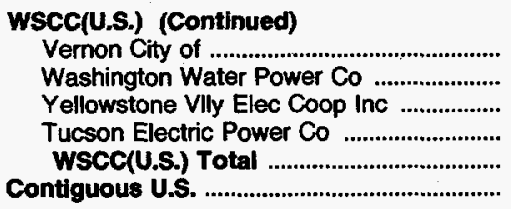 & $\begin{array}{l}\text { Publicly Owned } \\
\text { Investor-Owned } \\
\text { Cooperative } \\
\text { Investor-Owned }\end{array}$ & $\begin{array}{r}0 \\
370 \\
5 \\
8 \\
6,117 \\
19,238\end{array}$ & $\begin{array}{r}0 \\
14 \\
0 \\
40 \\
6,503 \\
16,550\end{array}$ & $\begin{array}{r}23 \\
7 \\
0 \\
0 \\
4,734 \\
8,644\end{array}$ & $\begin{array}{r}0 \\
0 \\
0 \\
0 \\
599 \\
842\end{array}$ & $\begin{array}{r}23 \\
391 \\
5 \\
47 \\
17,954 \\
45,275\end{array}$ \\
\hline 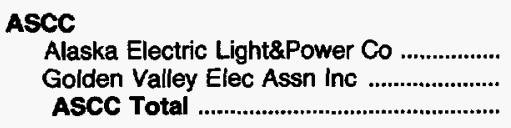 & $\begin{array}{l}\text { Investor-Owned } \\
\text { Cooperative }\end{array}$ & $\begin{array}{l}* \\
2 \\
2\end{array}$ & ${ }^{*} 0$ & $\begin{array}{l}0 \\
0 \\
0\end{array}$ & $\begin{array}{l}0 \\
0 \\
0\end{array}$ & * 2 \\
\hline 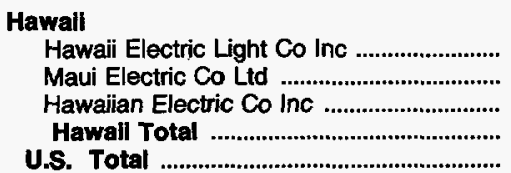 & $\begin{array}{l}\text { Investor-Owned } \\
\text { Investor-Owned } \\
\text { Investor-Owned }\end{array}$ & $\begin{array}{c}* \\
* \\
19,241\end{array}$ & $\begin{array}{r}2 \\
5 \\
10 \\
16 \\
16,567\end{array}$ & $\begin{array}{r}0 \\
0 \\
0 \\
0 \\
\mathbf{8 , 6 4 4}\end{array}$ & $\begin{array}{r}0 \\
0 \\
0 \\
0 \\
842\end{array}$ & $\begin{array}{r}2 \\
5 \\
10 \\
17 \\
45,294\end{array}$ \\
\hline
\end{tabular}

- Value less than 0.5

Notes: - Data are final. Data are provided for electric utilities with sales to ultimate consumers or sales for resale greater than or equal to 120,000 megawatthours. - Totals may not equal sum of components because of independent rounding.

Source: Energy Information Administration, Form EIA-861, "Annual Electric Utility Report." 



\section{Peakload Reduction}

One of the main goals of DSM programs is reduction in a utility's peak load. This can be achieved through the use of energy efficiency and load control programs. Peakload reduction (measured in megawatts (MW)) is categorized as potential or actual peakload reduction. Potential peakload reduction is the amount of load available for curtailment through load control programs such as direct load control, interruptible load control, other load management, or other DSM programs. Actual peakload reduction is the amount of reduction that is achieved from load control programs when they are actually put into force at the same time as peak load, and from the amount of reduction resulting from energy efficiency programs at the same time as peak load.

Utilities are required to report potential and actual peakload reductions on the Form EIA-861 for the direct load control, interruptible load control, other load management, and other DSM program categories. Utilities are also required to report actual peakload reductions from energy efficiency programs, but are not required to report potential peakload reduction for the energy efficiency category because these programs are focused on reducing energy consumption over many hours during the year and cannot be implemented specifically during the time of peak load. However, to allow for more accurate comparisons and data analyses to be conducted, in this publication it is assumed that potential peakload reduction resulting from energy efficiency programs was equal to actual peakload reduction. Only large utilities report annual effects for actual and potential peakload reductions; small utilities report only incremental reductions. ${ }^{11}$

\section{Annual Effects for Actual Peakload Reduction}

In 1993, actual peakload reduction was $23,069 \mathrm{MW}$, an increase of 85 percent since 1989 when EIA began its DSM data collection. Actual peakload reductions are predicted by utilities to increase to $25,266 \mathrm{MW}$ in 1994 and to $36,291 \mathrm{MW}$ in 1998 (Table 12).

For the 1993 reporting year, investor-owned utilities accounted for 71 percent of actual peakload reductions. Federally owned utilities accounted for 11 percent, followed by cooperatives with 10 percent, and publicly owned utilities with 8 percent. Utility forecasts indicated that investor-owned utilities are expected to increase actual peakload reductions by 13 percent in 1994 and increase at an annual rate of 10 percent through 1998. In 1998, cooperatives and Federally owned utilities are expected to provide almost 9 percent of actual peakload reductions and publicly owned utilities are expected to provide 8 percent (Table 12). ${ }^{12}$ Cooperatives have the greatest peakload reduction as a percentage of utility peak load because as purchasers of wholesale power, which is more expensive during peak periods, they focus on peakload reduction rather than energy savings. For this reason, it is economically efficient for cooperatives to reduce their system peak load as much as possible (Figure 5).

The 100 utilities with the greatest actual peakload reductions in 1993 accounted for 89 percent of the total peakload reduction. The 50 utilities with the greatest peakload reductions accounted for 76 percent of the total and the top 25 utilities accounted for 61 percent of actual peakload reductions (Figure 6). These 100, 50, and 25 utilities with the greatest actual peakload reductions represented 57,41 , and 27 percent, respectively, of total retail sales of electricity in the United States in 1993.

Energy efficiency programs accounted for the greatest share of actual peakload reductions, 45 percent of the 23,069 MW of total actual peakload reductions. Interruptible load, primarily an industrial sector program, contributed 29 percent of the total (Figure 7). Interruptible load programs increased 85 percent from 1992 to 1993. Direct load control programs accounted for 17 percent of actual peakload reduction. Other load management and other DSM programs combined for the remaining 9 percent of total peakload reductions (Table 13). The actual peakload reductions that are predicted for 1994 and 1998 indicated increases in all categories except other load management, where a decrease is predicted for 1994. The greatest increase is predicted for the energy efficiency category, an increase of 1,364 MW. This coincided with a large increase in energy savings resulting from energy efficiency programs. The greatest percentage of increase, 17 percent, is expected in the other DSM program category. From 1994 to 1998 , the average annual increase is expected to be approximately 9 percent, with the greatest average annual growth rate pre-

11 Incremental peakload reductions and energy savings are those caused by new programs and new participants in existing programs for the current reporting year.

12 Actual Peakload Reduction is a function of external factors such as weather conditions. Estimated predictions of Actual Peakload Reductions are dependent on certain conditions remaining static from year to year. In reality, utilities cannot predict weather conditions that may alter forecasted data. 
dicted for energy efficiency programs at 12 percent (Tables 13 and 18).

In 1993, the residential sector accounted for 38 percent of actual peakload reductions, the commercial sector, 33 percent, the industrial sector, 27 percent, and the other sector, 2 percent. The residential sector's share was greatest primarily because of the volume of participants in energy efficiency and direct load control programs. The greatest percentage of increases in actual peakload reductions from 1992 to 1993 were in the commercial and industrial sectors with 64 percent and 40 percent, respectively. The residential sector increased actual peakload reductions 16 percent and the other sector decreased 23 percent (Tables 14 and 20).

The NERC region with the greatest peakload reductions in 1993 was SERC with 36 percent of total U.S. peakload reductions, due partly to the inclusion of several large utilities that had the largest load management programs in the United States. The WSCC region had the second greatest peakload reductions contributing 18 percent of the total peakload reductions for 1993 . The greatest MW increase, 2,888 MW, occurred in the SERC region, and the greatest percentage of increase, 139 percent, occurred in the ERCOT region. For 1994, the MAAC region is predicted to increase by 54 percent. From 1994 to 1998, the SERC region is predicted to increase at annual rate of 17 percent (Table 18).

\section{Potential Peakload Reductions}

In 1993, potential peakload reductions increased 22 percent to 39,508 MW. For 1994, potential reductions are predicted to increase 7 percent to $42,220 \mathrm{MW}$, and by 1998 to increase at an average annual rate of 7 percent to $55,163 \mathrm{MW}$.

In 1993, investor-owned utilities accounted for 71 percent of the total potential peakload reduction, publicly owned accounted for 6 percent, cooperatives for 12 percent, and Federally owned for 11 percent. These utilities accounted for 67 percent (investorowned), 5 percent (publicly owned), 1 percent (cooperatives), and 2 percent (Federally owned) of the total retail sales of electricity in the United States. The greatest percentage of increase, 64 percent, was reported by Federally owned utilities due to an increase by the Tennessee Valley Authority in interruptible load programs. For 1994, an 8-percent increase is forecasted for investor-owned utilities. For 1998, publicly owned utilities are predicted to have the greatest annual rate of increase, 10 percent. Investor-owned utilities are predicted to continue to account for the greatest share of potential peakload reductions in 1998 at 73 percent.

Interruptible load programs accounted for $\mathbf{4 6}$ percent of potential peakload reductions in 1993; energy efficiency accounted for 26 percent, direct load control for 21 percent, and other load management and other DSM programs combined accounted for 7 percent. The greatest percentage of increase occurred for interruptible load programs. For 1994, energy efficiency programs are forecasted to increase 13 percent, and direct load programs are forecasted to increase 11 percent. For 1998, the greatest average annual increase, 12 percent, is predicted for energy efficiency programs. In 1998, the greatest share of potential peakload reduction is expected for interruptible load programs (Table 13).

The industrial sector accounted for 35 percent in 1993, the greatest share of potential peakload reductions, primarily as a result of interruptible load programs. The residential and commercial sectors contributed 33 percent and 30 percent, respectively, in 1993. The other sector accounted for less than 3 percent.

In 1993, the SERC region accounted for 37 percent of the total potential peakload reduction, primarily due to the inclusion of Tennessee Valley Authority, Carolina Power and Light, and Duke Power. The SERC region also accounted for the largest increase in potential peakload reduction from 1992 to 1993. The largest percentage of increase was achieved by the ERCOT region. The WSCC region is predicted to have the largest increase from 1993 to 1994, while the NPCC region is forecasted to have the largest percentage of increase in 1994. The ECAR region is forecasted to have the greatest average rate of increase through 1998.

\section{Incremental Effects for Actual Peakload Reduction}

In 1993, large utilities reported incremental peakload reductions of $4,648 \mathrm{MW}$, with investor-owned utilities contributing the greatest increase over 1992 at 755 MW. Publicly owned, large utilities actually decreased incremental peakload reductions by $55 \mathrm{MW}$ from 1992 to 1993. Small utilities reported incremental peakload reductions of $185 \mathrm{MW}$ for 1993, a decrease of $147 \mathrm{MW}$ from $332 \mathrm{MW}$ in 1992 (Table 15).

The greatest increases for large utilities in incremental peakload reduction from 1992 to 1993 by program category was for interruptible load and energy efficiency programs, which experienced almost equal incremental peakload reductions with $1,864 \mathrm{MW}$ and 1,839 MW, respectively. For small utilities, direct load control accounted for the greatest amount of incremental peakload reduction at $105 \mathrm{MW}$ (Table 16).

For large utilities, the industrial sector accounted for the greatest increase in incremental peakload reduction during 1993, $563 \mathrm{MW}$ more than in 1992. For small utilities, the residential sector accounted for the greatest amount, $76 \mathrm{MW}$, of peakload reduction (Table 17). 
Table 12. U.S. Electric Utility Actual and Potential Peakload Reductions by Class of Ownership, 1989 Through 1993, 1994, and 1998 (Megawatts)

\begin{tabular}{|c|c|c|c|c|c|c|c|}
\hline \multirow{2}{*}{ Class of Ownership } & \multicolumn{5}{|c|}{ Historical Actual Reductions } & \multicolumn{2}{|c|}{ Projected Actual Reductions } \\
\hline & 1989 & 1990 & 1991 & 1992 & 1993 & 1994 & 1998 \\
\hline \multirow[t]{3}{*}{ 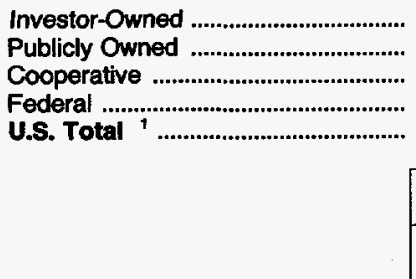 } & $\begin{array}{r}3,687 \\
1,054 \\
1,513 \\
1,209 \\
12,463\end{array}$ & $\begin{array}{r}9,435 \\
1,197 \\
1,822 \\
1,250 \\
13,704\end{array}$ & $\begin{array}{r}10,576 \\
1,634 \\
2,821 \\
588 \\
15,619\end{array}$ & $\begin{array}{r}12,330 \\
1,794 \\
2,374 \\
707 \\
17,204\end{array}$ & $\begin{array}{r}16,362 \\
1,898 \\
2,327 \\
2,481 \\
23,069\end{array}$ & $\begin{array}{r}18,463 \\
1,890 \\
2,444 \\
2,469 \\
25,266\end{array}$ & $\begin{array}{r}27,227 \\
2,801 \\
3,140 \\
3,122 \\
36,291\end{array}$ \\
\hline & \multicolumn{5}{|c|}{ Historical Potential Reductions } & \multicolumn{2}{|c|}{ Projected Potential Reductions } \\
\hline & 1989 & 1990 & 1991 & 1992 & 1993 & 1994 & 1998 \\
\hline 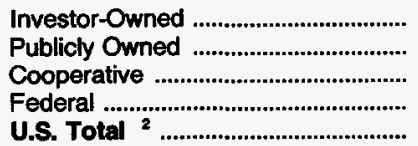 & $\begin{array}{l}\text { NA } \\
\text { NA } \\
\text { NA } \\
\text { NA } \\
\text { NA }\end{array}$ & $\begin{array}{l}\text { NA } \\
\text { NA } \\
\text { NA } \\
\text { NA } \\
\text { NA }\end{array}$ & $\begin{array}{l}\text { NA } \\
\text { NA } \\
\text { NA } \\
\text { NA } \\
\text { NA }\end{array}$ & $\begin{array}{r}23,774 \\
2,305 \\
3,669 \\
2,694 \\
32,442\end{array}$ & $\begin{array}{r}28,059 \\
2,376 \\
4,662 \\
4,411 \\
39,508\end{array}$ & $\begin{array}{r}30,347 \\
2,508 \\
4,889 \\
4,475 \\
42,220\end{array}$ & $\begin{array}{r}40,330 \\
3,624 \\
6,043 \\
5,166 \\
55,163\end{array}$ \\
\hline
\end{tabular}

1 Represents the sum of the actual peakload reductions attributable to direct load control, interruptible load, energy efficiency, other load management, and other demand-side management.

2 Represents the sum of the potential peakload reductions attributable to direct load control, interruptible load, other load management, other demand-side management, including the actual peakload reduction achieved by energy efficiency programs.

NA $=$ Data not available

Notes: -Data are final. •Data are provided for electric utilities with sales to ultimate consumers or sales for resale greater than or equal to 120,000 megawatthours. - Totals may not equal sum of components because of independent rounding.

Source; Energy Information Administration, Form EIA-861, "Annual Electric Utility Report." 
Figure 5. Actual Peakload Reductions as a Percentage of Total Peak Load by U.S. Electric Utilities with DSM Peakload Reduction Programs and by Class of Ownership, 1993

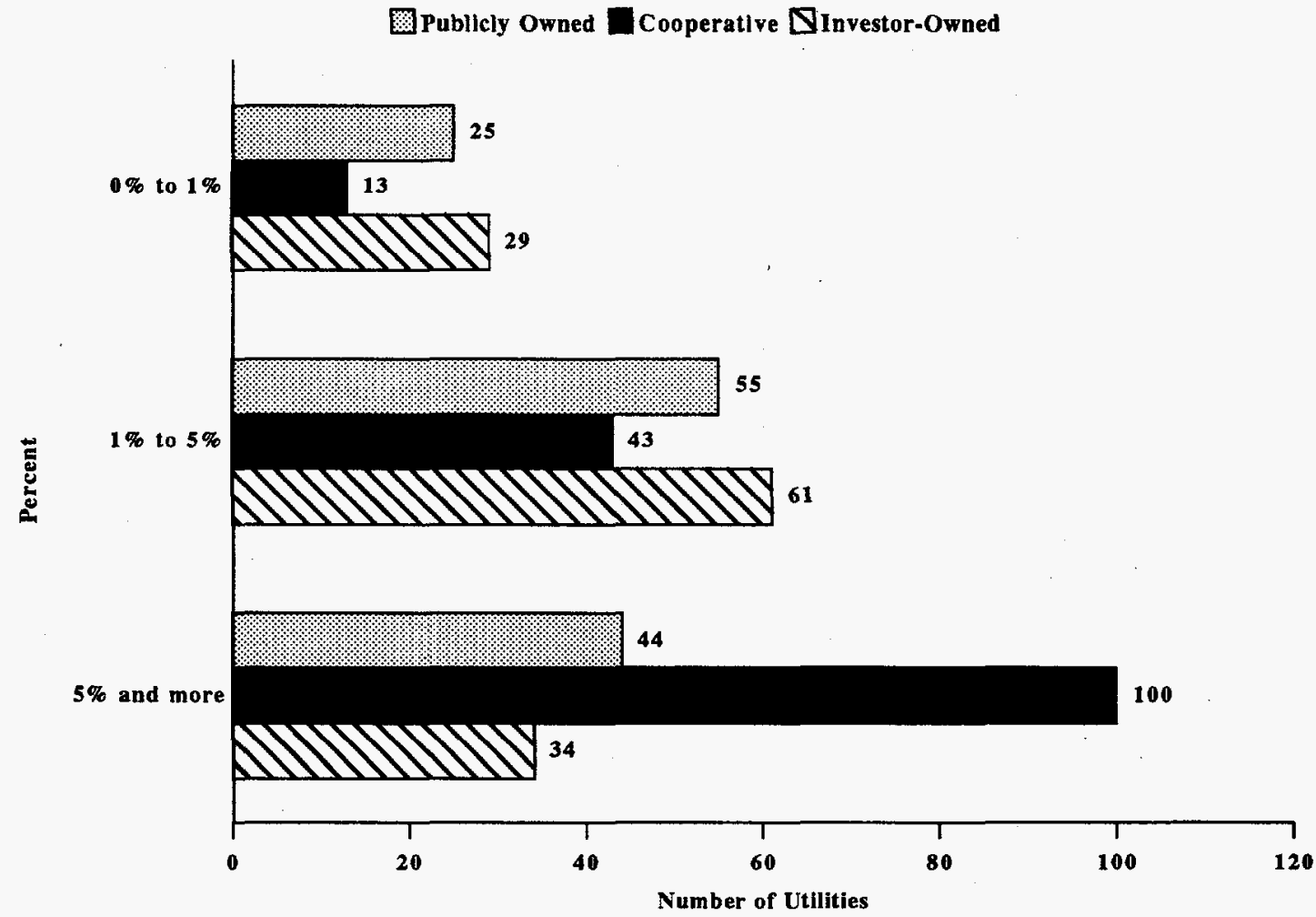

Note: This graph only includes utilities that reported peakload reductions.

Source: Energy Information Administration, Form EIA-861, "Annual Electric Utillty Report."

Figure 6. The Top 25, 50, and 100 U.S. Electric Utilities with the Greatest DSM Program Peakload Reductions by Class of Ownership, 1993

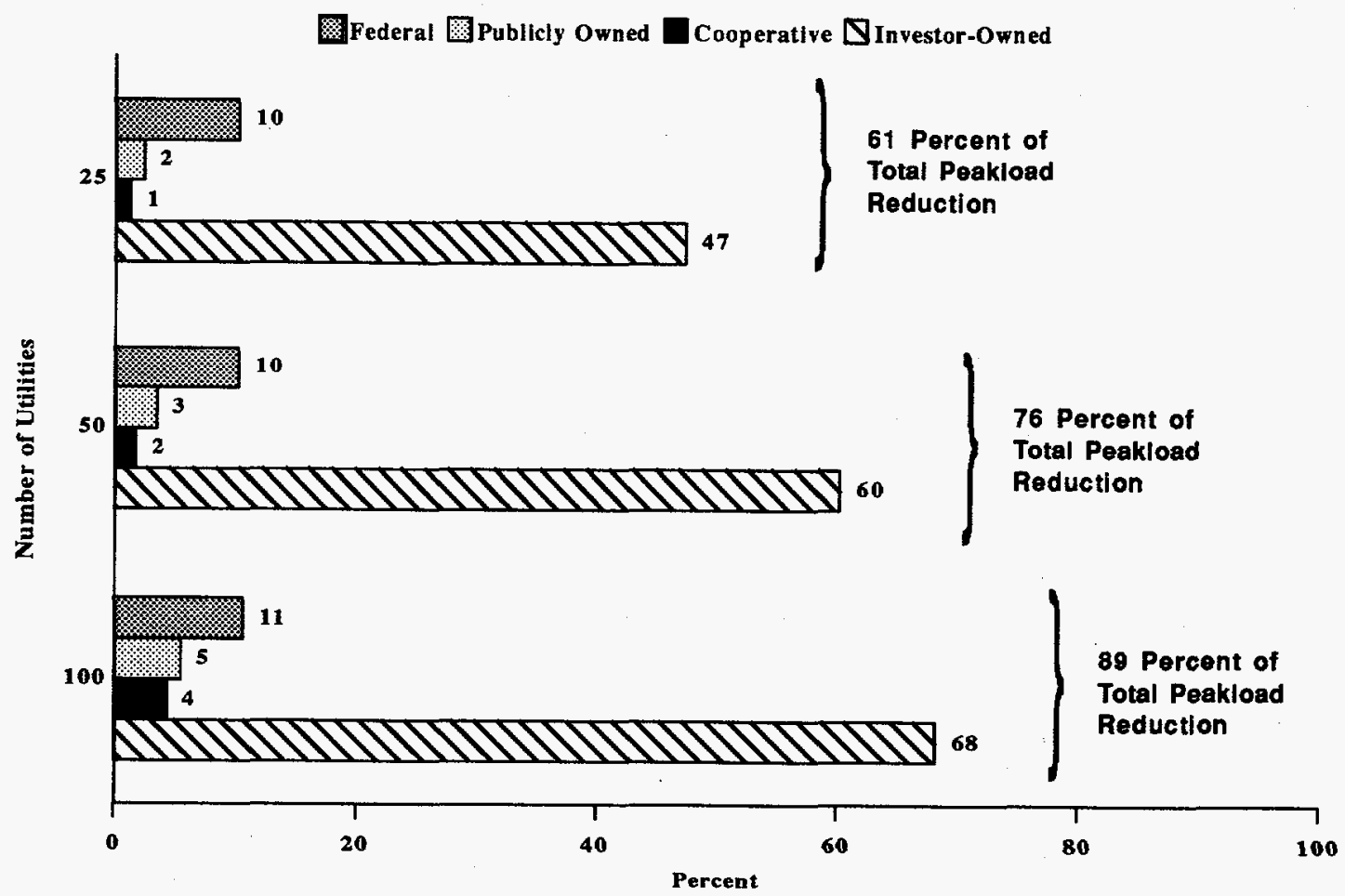

Note: Totals may not equal sum of components because of independent rounding.

Source: Energy Information Administration, Form EIA-861, "Annual Electric Utillty Report." 
Table 13. U.S. Electric Utility Actual and Potential Peakload Reductions by DSM Program Category, 1992, 1993, 1994, and 1998

(Megawatts)

\begin{tabular}{|c|c|c|}
\hline \multirow{2}{*}{ Program Category } & \multicolumn{2}{|c|}{ Historical Actual Reductions } \\
\hline & 1992 & 1993 \\
\hline 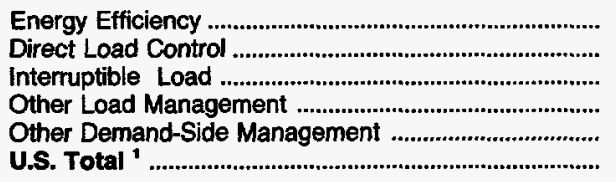 & $\begin{array}{r}7,890 \\
3,779 \\
3,579 \\
1,676 \\
281 \\
17,204\end{array}$ & $\begin{array}{r}10,368 \\
3,955 \\
6,628 \\
1,803 \\
315 \\
23,069\end{array}$ \\
\hline
\end{tabular}

\begin{tabular}{|r|r}
\hline \multicolumn{3}{c}{ Projected Actual Reductions } \\
\hline 1994 & 1998 \\
\hline 11,732 & 18,679 \\
4,168 & 5,625 \\
7,525 & 9,243 \\
1,471 & 2,249 \\
370 & 495 \\
25,266 & 36,291 \\
\hline
\end{tabular}

Energy Efficiency

Direct Load Control

Interruptible Load .

Other Load Management ...........

Other Demand-Side Management

U.S. Total ${ }^{1}$

\begin{tabular}{|r|r}
\hline \multicolumn{3}{|c}{ Historical Potential Reductions } \\
\hline 1992 & 1993 \\
\hline 7,890 & 10,368 \\
7,626 & 8,266 \\
14,566 & 18,235 \\
1,899 & 2,182 \\
461 & 457 \\
32,442 & 39,508 \\
\hline
\end{tabular}

Energy Efficiency

Direct Load Contro

Interruptible Load

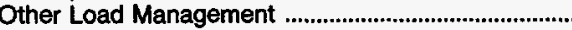

Other Demand-Side Management

U.S. Total ${ }^{2}$

\begin{tabular}{|l|l|}
\hline \multicolumn{3}{|c|}{ Projected Potential Reductions } \\
\hline 1994 & 1998 \\
\hline
\end{tabular}

Energy Efficiency

Direct Load Contro

Interruptible Load

Other Load Management

Lide Management

U.S. Total ${ }^{2}$

11,732
9,136
18,516
2,341
494
42,220

18,679
11,962
20,713
3,173
636
55,163

Represents the sum of the actual peakload reductions attributable to direct load control, interruptible load, energy efficiency, other load management, and other demand-side management.

2 Represents the sum of the potential peakload reductions attributable to direct load control, interruptible load, other load management, other demand-side management, including the actual peakload reduction achieved by energy efficiency programs.

Notes: -Data are final. $\bullet$ Data are provided for electric utilities with sales to ultimate consumers or sales for resale greater than or equal to 120,000 megawatthours. - Totals may not equal sum of components because of independent rounding.

Source: Energy Information Administration, Form ElA-861, "Annual Electric Utility Report." 
Figure 7. U.S. Electric Utility Actual and Potential Peakload Reductions by DSM Program Category, 1993

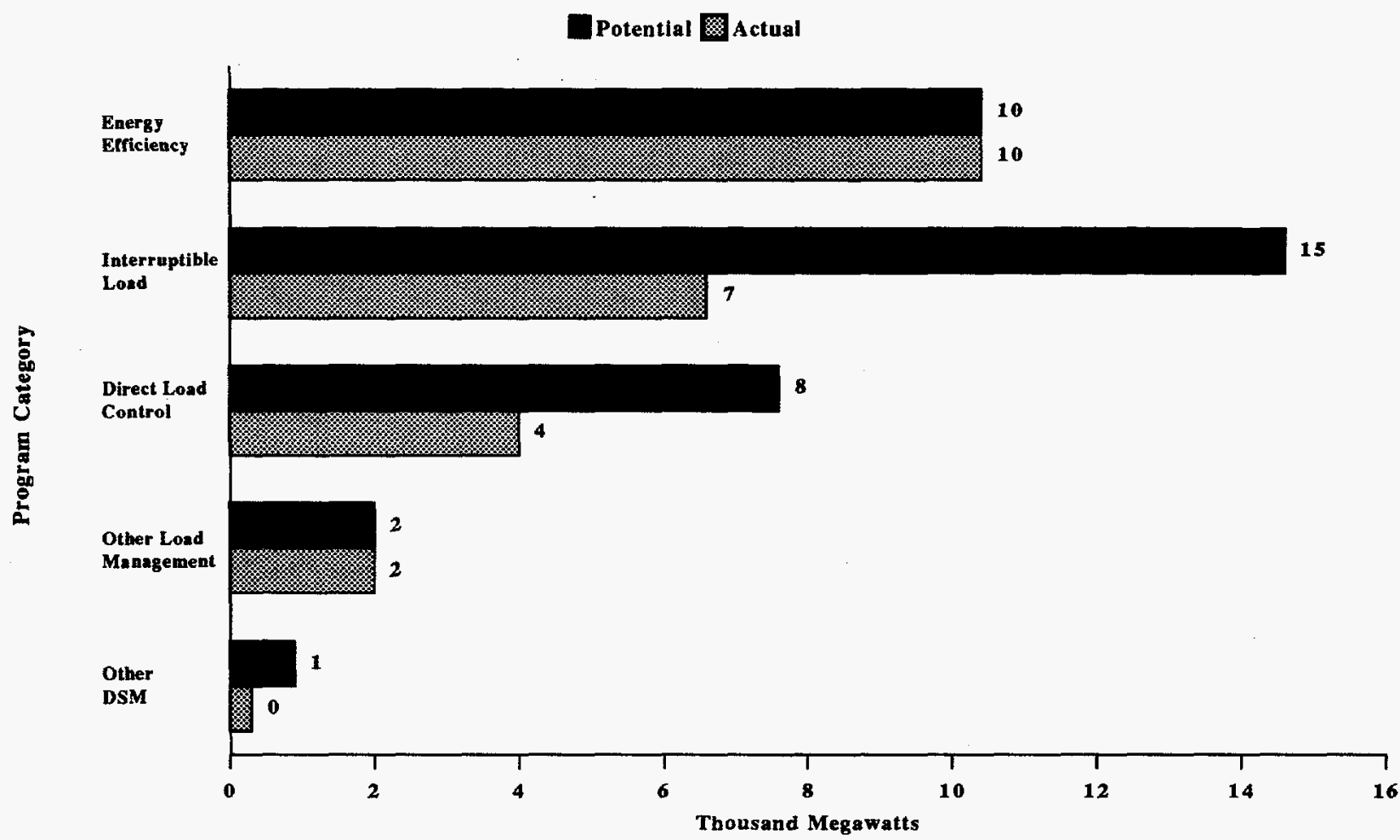

Source: Energy Information Administration, Form EIA-861, "Annual Electric Utility Report."

Table 14. U.S. Electric Utility Actual and Potential Peakload Reductions by Sector, 1992 and 1993

(Megawatts)

\begin{tabular}{|c|c|c|c|c|}
\hline \multirow{2}{*}{ Sectors } & \multicolumn{2}{|c|}{1992} & \multicolumn{2}{|c|}{1993} \\
\hline & Actual & Potential & Actual & Potential \\
\hline 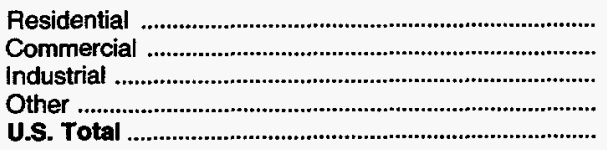 & $\begin{array}{r}7,606 \\
4,598 \\
4,467 \\
532 \\
17,204\end{array}$ & $\begin{array}{r}11,058 \\
7,002 \\
13,367 \\
1,014 \\
32,442\end{array}$ & $\begin{array}{r}8,851 \\
7,541 \\
6,270 \\
407 \\
23,069\end{array}$ & $\begin{array}{r}12,868 \\
11,821 \\
13,957 \\
862 \\
39,508\end{array}$ \\
\hline
\end{tabular}

Notes: -Data are final. -Data are provided for electric utilities with sales to ultimate consumers or sales for resale greater than or equal to 120,000 megawatthours. - Totals may not equal sum of components because of independent rounding.

Source: Energy Information Administration, Form EIA-861, "Annual Electric Utility Report." 
Table 15. U.S. Electric Utility Incremental Actual Peakload Reductions by Class of Ownership, 1992 and 1993

(Megawatts)

\begin{tabular}{|c|c|c|c|c|c|c|}
\hline \multirow{2}{*}{ Class of Ownership } & \multicolumn{2}{|c|}{ Large Utilities 1} & \multicolumn{2}{|c|}{ Small Utilities $^{2}$} & \multicolumn{2}{|c|}{ Total } \\
\hline & 1992 & 1993 & 1992 & 1993 & 1992 & 1993 \\
\hline 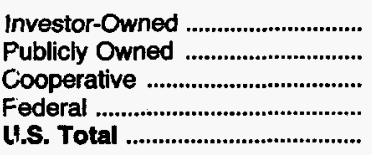 & $\begin{array}{r}3,222 \\
398 \\
290 \\
12 \\
3,922\end{array}$ & $\begin{array}{r}3,977 \\
343 \\
313 \\
14 \\
4,648\end{array}$ & $\begin{array}{r}* \\
165 \\
167 \\
0 \\
332\end{array}$ & $\begin{array}{r}* \\
95 \\
90 \\
0 \\
185\end{array}$ & $\begin{array}{r}3,222 \\
563 \\
457 \\
12 \\
4,254\end{array}$ & $\begin{array}{r}3,978 \\
438 \\
403 \\
14 \\
4,833\end{array}$ \\
\hline
\end{tabular}

1 Refers to electric utilities with sales to ultimate consumers or sales for resale greater than or equal to 120,000 megawatthours.

2 Refers to electric utilities with sales to ultimate consumers and sales for resale less than 120,000 megawatthours.

- Value less than 0.5 .

Notes: -Data are final. -Totals may not equal sum of components because of independent rounding.

Source: Energy Information Administration, Form EIA-861, "Annual Electric Utility Report."

Table 16. U.S. Electric Utility Incremental Actual Peakload Reductions by DSM Program Category, 1992 and 1993

(Megawatts)

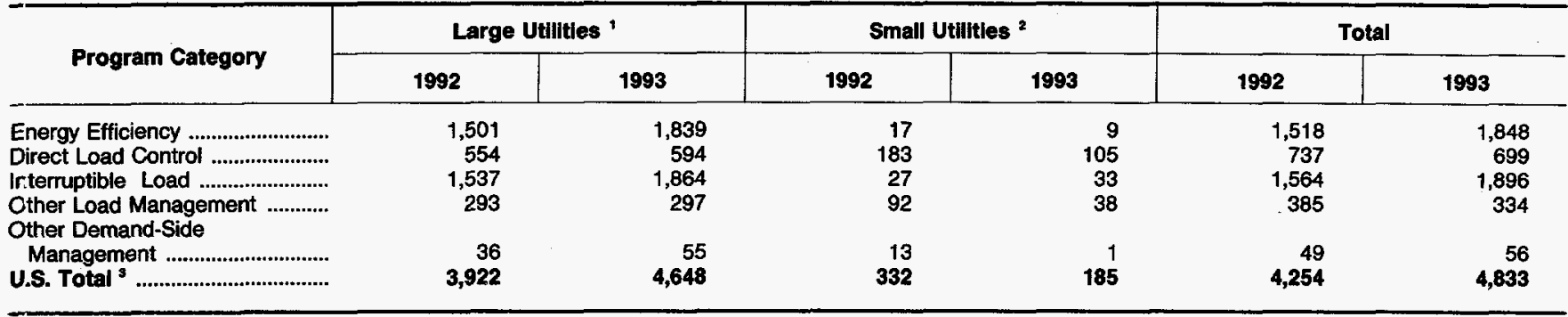

Refers to electric utilities with sales to ultimate consumers or sales for resale greater than or equal to 120,000 megawatthours.

2 Refers to electric utilities with sales to ultimate consumers and sales for resale less than 120,000 megawatthours.

- Represents the sum of the achieved reductions in the annual peakload attributable to direct load control, interruptible load, energy efficiency, other load management, and other demand-side management; whereas, in the Electric Power Annual, 1992,1992 and 1991 peakload reductions attributable to energy efficiency, other load management, and other demand-side management were reported together under conservation and other programs.

Notes: -Data are final. -Totals may not equal sum of components because of independent rounding.

Source: Energy Information Administration, Form ElA-861, "Annual Electric Utility Report."

T'able 17. U.S. Electric Utility Incremental Actual Peakload Reductions by Sector, 1992 and 1993

(Megawatts)

\begin{tabular}{|c|c|c|c|c|c|c|}
\hline \multirow{2}{*}{ Sector } & \multicolumn{2}{|c|}{ Large Utilities ' } & \multicolumn{2}{|c|}{ Small Utilities 2} & \multicolumn{2}{|c|}{ Total } \\
\hline & 1992 & 1993 & 1992 & 1993 & 1992 & 1993 \\
\hline 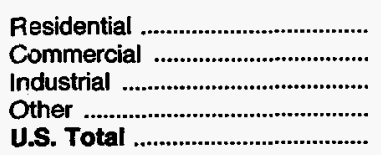 & $\begin{array}{r}1,112 \\
1,251 \\
1,451 \\
108 \\
3,922\end{array}$ & $\begin{array}{r}1,147 \\
1,427 \\
2,014 \\
61 \\
4,648\end{array}$ & $\begin{array}{r}139 \\
32 \\
113 \\
48 \\
332\end{array}$ & $\begin{array}{r}76 \\
35 \\
47 \\
28 \\
185\end{array}$ & $\begin{array}{r}1,251 \\
1,283 \\
1,564 \\
156 \\
4,254\end{array}$ & $\begin{array}{r}1,223 \\
1,462 \\
2,060 \\
88 \\
\mathbf{4 , 8 3 3}\end{array}$ \\
\hline
\end{tabular}

1 Refers to electric utilities with sales to ultimate consumers or sales for resale greater than or equal to 120,000 megawatthours.

2 Refers to electric utilities with sales to ultimate consumers and sales for resale less than 120,000 megawatthours.

Notes: -Data are final. -Totals may not equal sum of components because of independent rounding.

Source: Energy Information Administration, Form ElA-861, "Annual Electric Utility Report." 
Table 18. U.S. Electric Utility Actual and Potential Peakload Reductions by North American Electric Reliability Council Region and Hawaii by Utility, 1992, 1993, 1994, and 1998

(Megawatts)

\begin{tabular}{|c|c|c|c|c|c|c|c|c|}
\hline \multirow{3}{*}{$\begin{array}{c}\text { North American Electric Rellability } \\
\text { Council Reglon and Hawall / } \\
\text { Electric Utility }\end{array}$} & \multicolumn{4}{|c|}{ Historical Reductions } & \multicolumn{4}{|c|}{ Projected Reductions } \\
\hline & \multicolumn{2}{|c|}{1992} & \multicolumn{2}{|c|}{1993} & \multicolumn{2}{|c|}{1994} & \multicolumn{2}{|c|}{1998} \\
\hline & Actual & Potential & Actual & Potential & Actual & Potential & Actual & Potential \\
\hline \multicolumn{9}{|l|}{ ECAR } \\
\hline 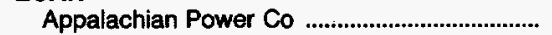 & 1 & 182 & 146 & 202 & 182 & 215 & 348 & 348 \\
\hline 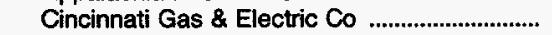 & 5 & 15 & 22 & 26 & 20 & 57 & 101 & 190 \\
\hline 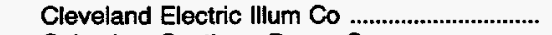 & 1 & 91 & 6 & 96 & 8 & 98 & 50 & 140 \\
\hline 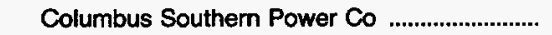 & 5 & 30 & 27 & 37 & 49 & 49 & 121 & 121 \\
\hline 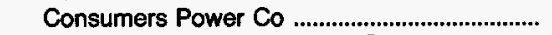 & 31 & 31 & 135 & 135 & 155 & 155 & 425 & 425 \\
\hline 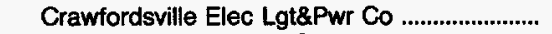 & - & - & 0 & 0 & 2 & 2 & 9 & 10 \\
\hline Dayton Power \& Light Co ................................... & 5 & 5 & 16 & 16 & 16 & 16 & 101 & 101 \\
\hline 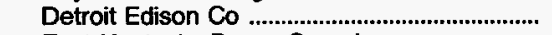 & 6 & 22 & 25 & 42 & 70 & 70 & 193 & 193 \\
\hline East Kentucky Power Coop Ine ........................ & 10 & 10 & 11 & 11 & 16 & 16 & 38 & 38 \\
\hline 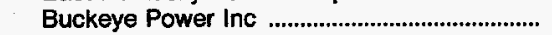 & 114 & 114 & 119 & 119 & 123 & 123 & 140 & 140 \\
\hline  & - & - & 0 & * & 0 & 2 & 0 & 4 \\
\hline Hendricks County Rural E M C ............................ & - & - & 4 & 4 & 0 & 6 & 0 & 10 \\
\hline 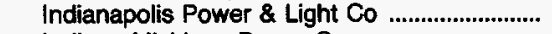 & 10 & 22 & 14 & 26 & 52 & 52 & 102 & 102 \\
\hline 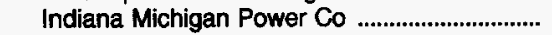 & 6 & 82 & 37 & 87 & 66 & 91 & 130 & 130 \\
\hline 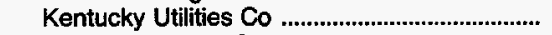 & 18 & 18 & 20 & 20 & 44 & 44 & 62 & 62 \\
\hline 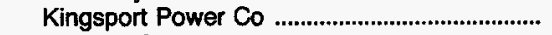 & * & * & 1 & 1 & 2 & 2 & 13 & 13 \\
\hline 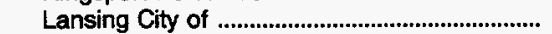 & - & - & * & 6 & * & 6 & * & 8 \\
\hline 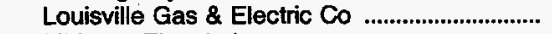 & 0 & 87 & 64 & 110 & 120 & 120 & 156 & 156 \\
\hline Midwest Electric Inc & 0 & 7 & 0 & 8 & 0 & 7 & 0 & 7 \\
\hline Monongahela Power Co .............................................. & 70 & 105 & 76 & 103 & 114 & 114 & 126 & 126 \\
\hline Northern Indiana Pub Serv Co .............................. & 0 & 121 & 0 & 121 & 0 & 121 & 0 & 135 \\
\hline 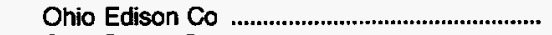 & 4 & 224 & 252 & 396 & 219 & 427 & 290 & 552 \\
\hline 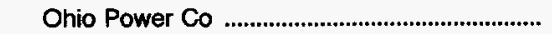 & 11 & 192 & 120 & 202 & 86 & 217 & 315 & 315 \\
\hline 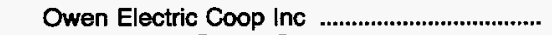 & * & * & 1 & 1 & 1 & 1 & 2 & 2 \\
\hline 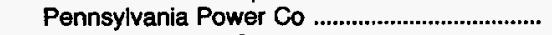 & 2 & 47 & 40 & 67 & 35 & 66 & 36 & 68 \\
\hline Potomac Edison Co & 148 & 154 & 158 & 165 & 174 & 179 & 242 & 242 \\
\hline PSI Energy Inc & 16 & 23 & 76 & 76 & 83 & 83 & 236 & 236 \\
\hline 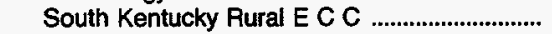 & 0 & 3 & - & - & - & - & - & - \\
\hline Southern Indiana Gas \& Elec Co ...................... & 2 & 32 & 13 & 45 & 58 & 58 & 108 & 108 \\
\hline South Central Power Co ........................................... & 22 & 22 & 25 & 25 & 25 & 25 & 0 & 30 \\
\hline 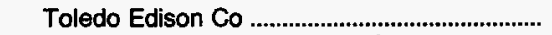 & 3 & 68 & 5 & 5 & 3 & 68 & 28 & 93 \\
\hline Utilities Dist-Western IN REMC ......................... & 4 & 4 & 11 & 19 & 1 & 1 & 3 & 3 \\
\hline Virginia Tech Electric Service ........................... & 2 & 2 & 2 & 2 & 2 & 2 & 3 & 3 \\
\hline 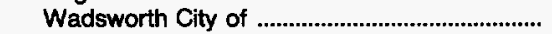 & - & - & 8 & 8 & 8 & 8 & 8 & 8 \\
\hline 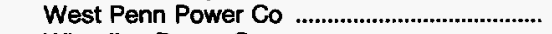 & 142 & 151 & 145 & 154 & 157 & 162 & 193 & 193 \\
\hline Wheeling Power Co & 1 & 1 & 3 & 3 & 3 & 3 & 7 & 7 \\
\hline 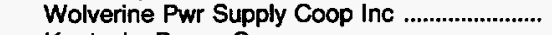 & 5 & 5 & 8 & 10 & 10 & 12 & 15 & 18 \\
\hline Kentucky Power Co ........... & * & 28 & 35 & 35 & 24 & 33 & 42 & 44 \\
\hline 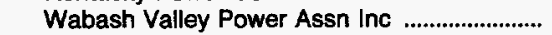 & 12 & 44 & 40 & 50 & 40 & 55 & 32 & 75 \\
\hline 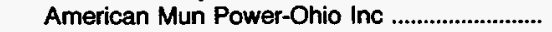 & 6 & & 6 & 9 & 7 & 9 & 8 & 10 \\
\hline 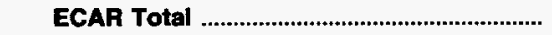 & 661 & 1,952 & 1,671 & 2,440 & 1,975 & 2,774 & 3,684 & 4,466 \\
\hline \multicolumn{9}{|l|}{ ERCOT } \\
\hline Austin City o & 195 & 242 & 212 & 259 & 235 & 282 & 328 & 365 \\
\hline Brazos Electric Power Coop Inc ............................ & & & 1 & 1 & 2 & 2 & 33 & 33 \\
\hline Bryan City of & 8 & 8 & 9 & 9 & 11 & 11 & 19 & 19 \\
\hline 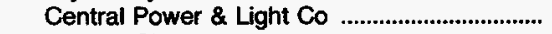 & 34 & 251 & 71 & 336 & 84 & 388 & 154 & 472 \\
\hline 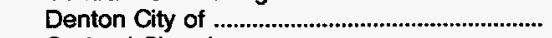 & 6 & 8 & 3 & 3 & 3 & 3 & 8 & 8 \\
\hline 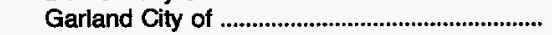 & 14 & 30 & 14 & 32 & 14 & 31 & 15 & 36 \\
\hline Georgetown Gity of & - & - & * & * & 1 & 2 & 4 & 6 \\
\hline Greenville Electric Util Sys ...................................... & 8 & 11 & 3 & 5 & 4 & 7 & 7 & 11 \\
\hline Guadalupe Valley Elec Coop Inc ....................... & 61 & 66 & 61 & 68 & 61 & 68 & 62 & 69 \\
\hline 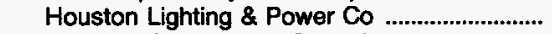 & 42 & 897 & 48 & 1,176 & 74 & 929 & 145 & 814 \\
\hline Johnson County Elec Coop Assn ...................... & * & * & 2 & & 2 & 2 & 3 & 3 \\
\hline Lower Colorado River Authority ............................. & 68 & 91 & 92 & 92 & $9 \overline{6}$ & 96 & 134 & 134 \\
\hline Magic Valley Electric Coop Inc .......................... & - & - & ^ & * & 1 & 1 & 1 & 1 \\
\hline 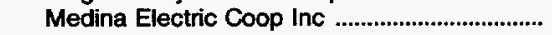 & 9 & 28 & 6 & 28 & 8 & 28 & 6 & 22 \\
\hline San Antonio Public Service Bd ............................... & 13 & 14 & 0 & 0 & 0 & 0 & 0 & 0 \\
\hline San Bernard Electric Coop Inc .............................. & 3 & 3 & 14 & 27 & 10 & 22 & 11 & 24 \\
\hline 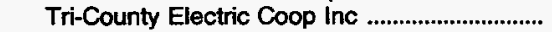 & 2 & 2 & 2 & 2 & 3 & 3 & 3 & 3 \\
\hline 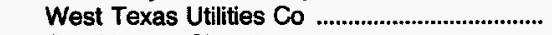 & 0 & 24 & 39 & 53 & 0 & 58 & 0 & 62 \\
\hline San Marcos City of ............................................... & 3 & 12 & 3 & 12 & * & * & $*$ & * \\
\hline Texas-New Mexico Power Co ............................ & 60 & 108 & 19 & 26 & 21 & 28 & 26 & 36 \\
\hline 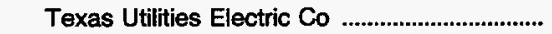 & 65 & 528 & 814 & 1,314 & 569 & 1,069 & 569 & 1,069 \\
\hline 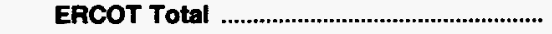 & 592 & 2,325 & 1,414 & 3,446 & 1,199 & 3,029 & 1,528 & 3,187 \\
\hline
\end{tabular}

See footnotes at end of table. 
Table 18. U.S. Electric Utility Actual and Potential Peakload Reductions by North American Electric Reliability Council Region and Hawaii by Utility,

1992, 1993, 1994, and 1998

(Megawatts) (Continued)

\begin{tabular}{c|c|c|c|c|c|c|c|c}
\hline \multirow{2}{*}{$\begin{array}{c}\text { North American Electric Reliability } \\
\text { Council Region and Hawall } / \\
\text { Electric Utility }\end{array}$} & \multicolumn{3}{|c|}{ Historical Reductions } & \multicolumn{4}{c}{ Projected Reductions } \\
\cline { 2 - 7 } & \multicolumn{2}{|c|}{1992} & \multicolumn{2}{|c|}{1993} & \multicolumn{2}{|c|}{1994} & 1998 \\
\cline { 2 - 7 } & Actual & Potential & Actual & Potential & Actual & Potential & Actual & Potential \\
\hline
\end{tabular}

MAAC

A \& N Electric Coop

Allegheny Electric Coop Inc

Atlantic City Electric Co

Baltimore Gas \& Electric Co ...............................

Choptank Electric Coop Inc

Conowingo Power Co

Delmarva Power \& Light Co

Delaware Electric Coop Inc

Easton Utilities Comm

Metropolitan Edison Co

Pennsylvania Electric Co......

Potomac Electric Power Co

Southern Maryland El Coop Inc

Somerset Rural Elec Coop Inc

Adams Electric Coop Inc

Bedford Rural Elec Coop Inc

Central Electric Coop Inc

Claverack Rural Elec Coop Inc

Southwest Central R E C Corp

MAAC Total

\begin{tabular}{rrr}
1 & 1 & \\
101 & 237 & 94 \\
103 & 103 & 5 \\
353 & 389 & \\
1 & 3 & \\
3 & 3 & 16 \\
104 & 230 & \\
5 & 13 & -14 \\
- & 110 & 144 \\
73 & 375 & 27 \\
375 & 40 & 51 \\
40 & 106 & 167 \\
90 & 309 & 45 \\
45 & 339 & 17 \\
160 & 181 & 244 \\
170 & 37 & 32 \\
34 & 2 & \\
1 & - & \\
7 & 5 & \\
\hline 3 & 3 & \\
2 & 6 & \\
$*$ & 2,500 & 1,493 \\
1,677 & &
\end{tabular}

\begin{tabular}{r}
1 \\
\hline 94 \\
59 \\
5 \\
3 \\
162 \\
6 \\
$*$ \\
144 \\
276 \\
51 \\
167 \\
45 \\
177 \\
244 \\
32 \\
1 \\
8 \\
2 \\
3 \\
4 \\
5 \\
0 \\
5 \\
1,493
\end{tabular}

$\begin{array}{rr}1 & 1 \\ - & - \\ 94 & 105 \\ 523 & 85 \\ 10 & 6 \\ 3 & 1 \\ 246 & 16 \\ 13 & 6 \\ * & 1 \\ 183 & 173 \\ 276 & 275 \\ 51 & 62 \\ 167 & 242 \\ 370 & 370 \\ 375 & 534 \\ 368 & 346 \\ 185 & 49 \\ 2 & 3 \\ 10 & 15 \\ 2 & 2 \\ 4 & 3 \\ 4 & 4 \\ 5 & 0 \\ * & 0 \\ 6 & 5 \\ 2,899 & 2,303\end{array}$

2
105
648
11
1
237
13
1
212
275
62
242
370
534
427
211
3
17
2
5
4
6
1
6
3,394

$\begin{array}{rr}1 & \\ 137 & 137 \\ 170 & 888 \\ 7 & 13\end{array}$

2

MAIN

Boone Electric Coop

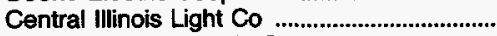

Coles-Moultrie Electric Coop

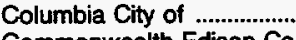

Commonwealth Edison $\mathrm{Co}$.

Corn Belt Electric Coop Inc ...

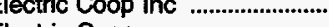

Illinois Power $\mathrm{Co}$

Madison Gas \& Electric Co

Manitowoc Public Utilities

Marshfield City of

Menard Electric Coop...



Southeastern IL Elec Coop Inc

Southwestem Electric Coop Inc

Springfield City of .

Tri-County Electric Coop Inc

Union Electric Co

Wayne-White Counties Elec Coop ....................

Wisconsin Electric Power Co ...

Wisconsin Power \& Light Co .......

Wisconsin Public Power Inc Sys

Wisconsin Public Service Corp

MAIN Total

$\begin{array}{rrrr}* & * & 2 & 7 \\ 54 & 61 & 62 & 62 \\ 2 & 2 & 8 & 8 \\ 11 & 20 & 7 & 17 \\ 16 & 171 & 23 & 173 \\ 9 & 15 & 6 & 15 \\ 3 & 5 & 6 & 9 \\ 2 & 9 & 10 & 12 \\ 204 & 204 & 5 & 166 \\ 13 & 19 & 30 & 42 \\ 1 & 1 & 2 & 2 \\ * & 1 & 1 & 1 \\ 0 & 16 & 0 & * \\ 0 & 4 & 8 & 8 \\ * & * & 0 & * \\ 3 & 12 & 25 & 50 \\ 5 & 5 & 5 & 9 \\ 13 & 18 & 12 & 12 \\ 190 & 247 & 145 & 182 \\ 0 & 12 & 0 & 12 \\ 205 & 292 & 336 & 435 \\ 44 & 163 & 54 & 196 \\ 2 & 2 & 7 & 7 \\ 62 & 84 & 91 & 121 \\ 840 & 1,365 & 844 & 1,545\end{array}$

$\begin{array}{rrrr}2 & 7 & 3 & 13 \\ 0 & 77 & 5 & 11 \\ 9 & 9 & 11 & 11 \\ 13 & 31 & 21 & 53\end{array}$

$\begin{array}{llll}171 & 171 & 605 & 605\end{array}$

$\begin{array}{rrrr}9 & 16 & 15 & 24 \\ 7 & 9 & 10 & 12\end{array}$

1212

$5 \quad 158$

$\begin{array}{rr}44 & 62 \\ 1 & 1\end{array}$

1

11
0

22
6

6
12
0

9

314

223

235

1,122

\begin{tabular}{rrrr}
0 & 6 & $*$ & 6 \\
3 & 3 & - & - \\
-4 & - & $*$ & 5 \\
7 & 7 & 5 & 6 \\
5 & 8 & 6 & 15 \\
0 & 17 & 4 & 5 \\
\hline 15 & -22 & 5 & 27 \\
& 22 & 15 & 22
\end{tabular}

MAPP(U.S.)

Ames City of

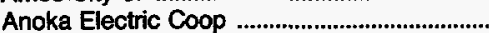

Anoka City of

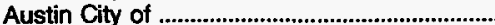

Barron Electric Coop .............................................

Beatrice City of

Cedar Falls City of

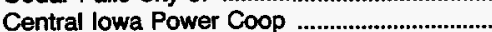

Central Power Elec Coop Inc

See footnotes at end of table. 
Table 18. U.S. Electric Utility Actual and Potential Peakload Reductions by North American Electric Reliability Council Region and Hawaii by Utility,

1992, 1993, 1994, and 1998

(Megawatts) (Continued)

\begin{tabular}{|c|c|c|c|c|c|c|c|c|}
\hline \multirow{3}{*}{$\begin{array}{l}\text { North American Electric Reliability } \\
\text { Council Region and Hawall / } \\
\text { Electric Utillty }\end{array}$} & \multicolumn{4}{|c|}{ Historical Reductions } & \multicolumn{4}{|c|}{ Projected Reductions } \\
\hline & \multicolumn{2}{|c|}{1992} & \multicolumn{2}{|c|}{1993} & \multicolumn{2}{|c|}{1994} & \multicolumn{2}{|c|}{1998} \\
\hline & Actual & Potential & Actual & Potential & Actual & Potential & Actual & Potential \\
\hline \multicolumn{9}{|l|}{ MAPP(U.S.) (Continued) } \\
\hline 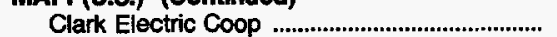 & 4 & 4 & 4 & 4 & 3 & 3 & 3 & 3 \\
\hline 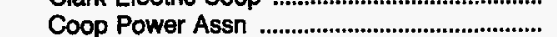 & $*$ & 89 & 2 & 110 & 5 & 129 & 16 & 209 \\
\hline Cornhusker Public Power Dist ......................... & 14 & 20 & 6 & 23 & 7 & 23 & 10 & 25 \\
\hline 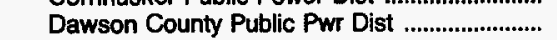 & 33 & 35 & * & $\star$ & 0 & 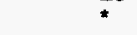 & 0 & 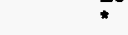 \\
\hline 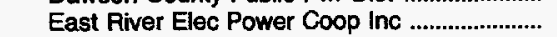 & 40 & 72 & 43 & 104 & 32 & 94 & 36 & 101 \\
\hline Fairmont Public Utilities Comm ............................. & 2 & 6 & $*$ & $\star$ & 1 & 1 & 1 & 2 \\
\hline Freeborn-Mower Electric Coop ............................ & - & - & 3 & 3 & 4 & 4 & 9 & 9 \\
\hline Grant-Lafayette Electric Coop .......................... & 4 & 6 & 5 & 5 & 5 & 5 & 9 & 9 \\
\hline IES Utilities Inc & - & - & 178 & 339 & 366 & 366 & 463 & 463 \\
\hline 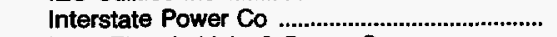 & 2 & 26 & 38 & 51 & 61 & 61 & 107 & 107 \\
\hline lowa Electric Light \& Power Co ......................... & 81 & 203 & - & - & - & - & - & - \\
\hline 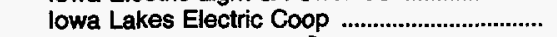 & 7 & 7 & 12 & 13 & 15 & 23 & 21 & 29 \\
\hline lowa Southern Utilities Co .................................... & 1 & 2 & - & - & - & - & - & - \\
\hline lowa-Illinois Gas\&Electric Co ............................... & - & - & 0 & 0 & 7 & 7 & 24 & 24 \\
\hline 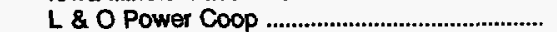 & 2 & 2 & 2 & 2 & 2 & 2 & 2 & 2 \\
\hline 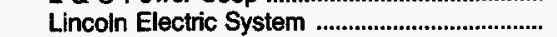 & $\overline{1}$ & 1 & $\overline{1}$ & 2 & 2 & 2 & 3 & 4 \\
\hline Linn County Rural E C A ...................................... & * & $\star$ & - & - & - & - & - & - \\
\hline Loup River Public Power Dist ........................... & 1 & 12 & -- & - & - & - & - & - \\
\hline 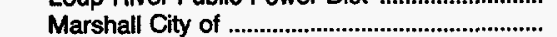 & 3 & 5 & 3 & 5 & 3 & 6 & 3 & 7 \\
\hline 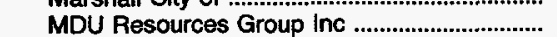 & 5 & 9 & 7 & 11 & 12 & 13 & 15 & 17 \\
\hline 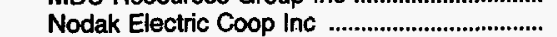 & 62 & 62 & 61 & 61 & 63 & 63 & 68 & 68 \\
\hline Midland Power Coop & $*$ & 2 & $*$ & $*$ & $*$ & 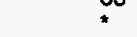 & $*$ & 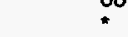 \\
\hline Maquoketa Valley Rrl Elec Coop ...................... & * & * & - & - & - & - & - & 一 \\
\hline 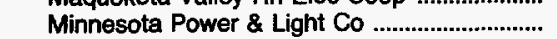 & 14 & 82 & 16 & 102 & 123 & 214 & 165 & 278 \\
\hline 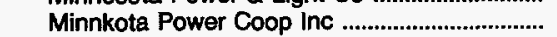 & 275 & 275 & 285 & 285 & 290 & 290 & 310 & 310 \\
\hline 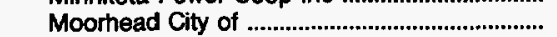 & 1 & 1 & 12 & 12 & 14 & 14 & 18 & 21 \\
\hline Nebraska Public Power District ......................... & 8 & 8 & 7 & 9 & 7 & 9 & 7 & 9 \\
\hline Norris Public Power District ................................ & 8 & 20 & 1 & 8 & 8 & 8 & 9 & 9 \\
\hline 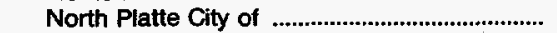 & 4 & 7 & 7 & 8 & 8 & 9 & 8 & 9 \\
\hline Northern States Power Co ...................................... & 48 & 93 & 126 & 126 & 126 & 146 & 185 & 215 \\
\hline 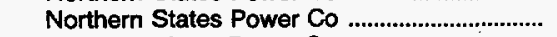 & 550 & 550 & 691 & 691 & 841 & 841 & 1,375 & 1,375 \\
\hline Northwest lowa Power Coop ............................. & 4 & 24 & 7 & 27 & 12 & 28 & 14 & 32 \\
\hline Northwestern Public Service Co ........................ & * & * & $*$ & $*$ & $\star$ & * & 1 & 1 \\
\hline Oakdale Electric Coop ....................................... & 7 & 9 & 4 & 4 & 4 & 4 & 4 & 4 \\
\hline 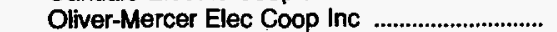 & 2 & 3 & 2 & 2 & 2 & 2 & 2 & 2 \\
\hline Omaha Public Power District ................................. & 1 & 1 & 2 & 2 & $1 \overline{8}$ & $1 \overline{8}$ & $\overline{51}$ & 51 \\
\hline 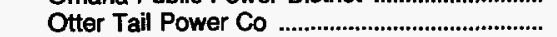 & 6 & 239 & 90 & 110 & 8 & 112 & 8 & 111 \\
\hline Owatonna City of & 7 & 20 & 9 & 20 & 5 & 15 & 5 & 15 \\
\hline 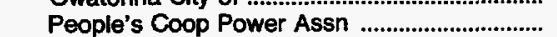 & 2 & 3 & 4 & 4 & 5 & 5 & 6 & 6 \\
\hline 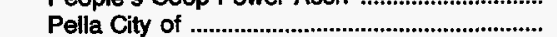 & - & $\ldots$ & $*$ & $*$ & $*$ & 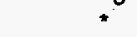 & * & $*$ \\
\hline 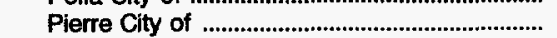 & 6 & 11 & 5 & 8 & 6 & 9 & 8 & 11 \\
\hline Polk-Burnett Electric Coop ..................................... & 8 & 8 & 8 & 16 & 9 & 17 & 10 & 20 \\
\hline 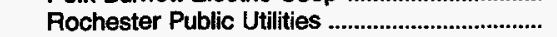 & 6 & 8 & 8 & 9 & 8 & 9 & 13 & 14 \\
\hline 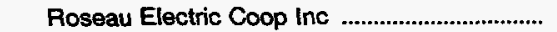 & - & - & 19 & 19 & 20 & 20 & 25 & 25 \\
\hline 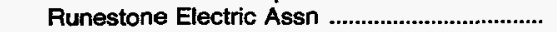 & 8 & 17 & 6 & 22 & 6 & 23 & 7 & 25 \\
\hline Shakopee Public Utilities Comm ...................... & 0 & 1 & 1 & 1 & 1 & 1 & 2 & 3 \\
\hline Superior Water Light\&Power Co ...................... & 3 & 3 & * & $t$ & 6 & 6 & 8 & 8 \\
\hline 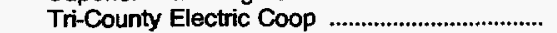 & 10 & 15 & 9 & 9 & 9 & 9 & 10 & 10 \\
\hline 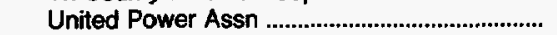 & 95 & 193 & 106 & 158 & 111 & 165 & 123 & 184 \\
\hline 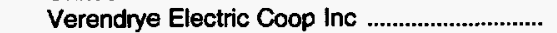 & 4 & 10 & 4 & 4 & 5 & 5 & 6 & 6 \\
\hline 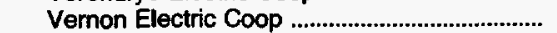 & - & - & 7 & 7 & 6 & 6 & 6 & 6 \\
\hline 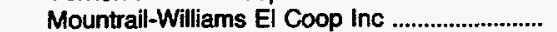 & 5 & 9 & 5 & 10 & 5 & 10 & 6 & 12 \\
\hline Wild Rice Electric Coop Inc ................................. & 13 & 15 & 19 & 52 & 19 & 53 & 21 & 58 \\
\hline Municipal Energy Agency of NE ....................... & 18 & 18 & 20 & 23 & 14 & 18 & 19 & 23 \\
\hline Midwest Power Systems Inc ................................ & 83 & 83 & 179 & 179 & 158 & 158 & 243 & 243 \\
\hline Cass County Electric Coop Inc ........................... & 52 & 55 & 57 & 61 & 55 & 58 & 61 & 65 \\
\hline 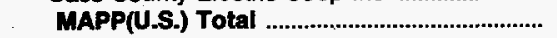 & 1,542 & 2,405 & 2,121 & 2,809 & 2,538 & 3,177 & 3,581 & 4,346 \\
\hline \multicolumn{9}{|l|}{ NPCC(U.S.) } \\
\hline 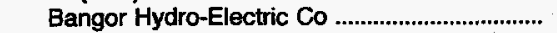 & 7 & 8 & 7 & 7 & 10 & 10 & 14 & 14 \\
\hline Blackstone Valley Electric Co .............................. & 8 & 8 & 12 & 12 & 9 & 10 & 15 & 16 \\
\hline 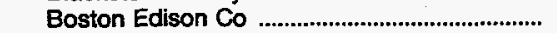 & 113 & 113 & 101 & 101 & 24 & 34 & 10 & 20 \\
\hline Braintree Town of & 0 & 2 & 3 & 7 & 4 & 7 & 5 & 6 \\
\hline 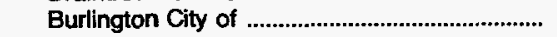 & 5 & 5 & 3 & 3 & 3 & 3 & 3 & 3 \\
\hline
\end{tabular}

See footnotes at end of table. 
Table 18. U.S. Electric Utility Actual and Potential Peakload Reductions by North American Electric Reliability Council Region and Hawaii by Utility, 1992, 1993, 1994, and 1998

(Megawatts) (Continued)

\begin{tabular}{l|c|c|c|c|c|c|c|c}
\hline \multirow{2}{*}{$\begin{array}{c}\text { North American Electric Rellability } \\
\text { Councll Region and Hawall } / \\
\text { Electric Utilty }\end{array}$} & \multicolumn{3}{|c|}{ Historical Reductions } & \multicolumn{4}{c}{ Projected Reductions } \\
\cline { 2 - 7 } & \multicolumn{2}{|c|}{1992} & \multicolumn{2}{|c|}{1993} & \multicolumn{3}{c|}{1994} & 1998 \\
\cline { 2 - 8 } & Actual & Potential & Actual & Potential & Actual & Potential & Actual & Potential \\
\hline
\end{tabular}

NPCC(U.S.) (Continued)

Cambridge Electric Light Co

Central Hudson Gas \& Elec Corp .....................

Central Maine Power Co

Central Vermont Pub Serv Corp

Chicopee City of

Citizens Utilities Co

Commonwealth Electric $\mathrm{Co}$

Concord Electric Co

Connecticut Light \& Power Co

Connecticut Valley Elec Co Inc

Consolidated Edison Co-NY Inc

Eastern Edison Co

Exeter \& Hampton Electric Co

Fitchburg Gas \& Elec Light Co

Green Mountain Power Corp

Holyoke City of

Hingham City of .

Jamestown City of

Littleton Town of ...........

Long Island Lighting Co

Maine Public Service Co

Massachusetts Electric Co

Massena Town of

Montaup Electric

Narragansett Electric Co

New England Power Co

New Hampshire Elec Coop Inc ......

New York State Elec \& Gas Corp ...................

Newport Electric Corp .

Niagara Mohawk Power Corp

Norwood City of .

Power Authority of State of NY.........

Public Service Co of NH

Reading Town of

Rochew

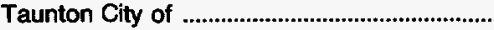

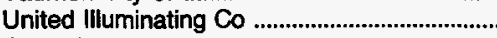

Omya Inc

Wellesley Town of

Granite State Electric Co

NPCC(U.S.) Total

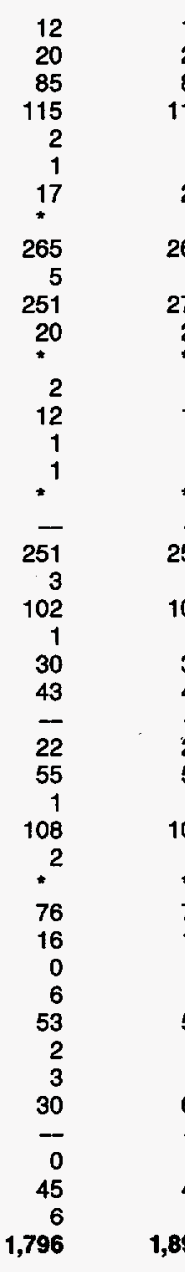

$\begin{array}{rr}12 & 28 \\ 85 & 84 \\ 115 & 122\end{array}$

$6 \quad 15$

$23 \quad 35$

122

2

21

1

5

271

20

2
16

1
7

251

6

102

30

43

24

55
1

1
108

2

76

16

0
8
53

53
2

3
69

$\frac{69}{0}$

0
45

6
890

267

184

25

1
2
15

4

0
194

194
1

147

32

56
0

31

31
157

135

135

1
98

33

* 6

49
3

4
57

* 0

59
8
968

84
122
1

7
19

1
267

267
5

213

25

1
2
21

21
1
7

* 1

194

6
147

4

32

56
71

39

158

2
135

$\frac{1}{7}$

98

33

8

49

4
57

*

59
8

2,130

5

29

89

160

2

11
13

1
272

272
5

530

17

1
3

19

19
4

Aiken Electric Coop Inc

Alabama Electric Coop Inc

Alabama Power Co

Albemarle City of

Altamaha Electric Member Corp

Athens City of

Berkeley Electric Coop Inc

Black River Electric Coop Inc ............................

Blue Ridge Elec Member Corp

Bristol City of

Canoochee Electric Member Corp

Carolina Power \& Light Co

Carroll Electric Member Corp ...

Carteret-Craven El Member Corp

Central Florida Elec Coop Inc

\begin{tabular}{rr}
3 & \\
5 & 107 \\
154 & 634 \\
\hline 3 & \\
1 & \\
- & \\
2 & \\
18 & \\
5 & \\
0 & \\
\hline 2 & \\
740 & \\
13 & \\
8 & \\
0 &
\end{tabular}

See footnotes at end of table. 
Table 18. U.S. Electric Utility Actual and Potential Peakload Reductions by North American Electric Reliability Council Region and Hawaii by Utility,

1992, 1993, 1994, and 1998

(Megawatts) (Continued)

\begin{tabular}{|c|c|c|c|c|c|c|c|c|}
\hline \multirow{3}{*}{$\begin{array}{c}\text { North American Electric Reliability } \\
\text { Council Region and Hawall./ } \\
\text { Electric Utillty }\end{array}$} & \multicolumn{4}{|c|}{ Historical Reductions } & \multicolumn{4}{|c|}{ Projected Reductions } \\
\hline & \multicolumn{2}{|c|}{1992} & \multicolumn{2}{|c|}{1993} & \multicolumn{2}{|c|}{1994} & \multicolumn{2}{|c|}{1998} \\
\hline & Actual & Potential & Actual & Potential & Actual & Polential & Actual & Potential \\
\hline
\end{tabular}

SERC (Continued)

Central Georgia El Member Corp

Central Electric Member Corp

Clay Electric Coop Inc

Coast Electric Power Assn

Coastal Electric Member Corp .

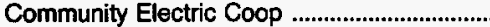

Coweta-Fayette El Member Corp .....................

Crescent Electric Member Corp

Crisp County Power Comm

Davidson Electric Member Corp ........................

Dothan City of

Douglas City of

Duke Power

East Point City of

Elizabeth City City of

Fairfield Electric Coop Inc

Fayetteville Public Works Comm

Flint Electric Membership Corp

Florida Keys El Coop Assn Ine

Florida Power \& Light Co .

Fort Pierce Utilities Auth

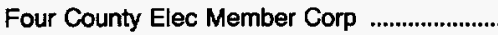

Gaffney City of .

Gainesville Regional Utilities

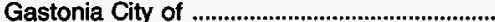

GreyStone Power Corp ........................................

Georgia Power Co

Grady County Elec Member Corp

Griffin City of

Gulf Power Co

Harrisonburg City of

Hart Electric Member Corp

Haywood Electric Member Corp

High Point Town of

Jackson Electric Member Corp ........................

Jacksonville Electric Auth

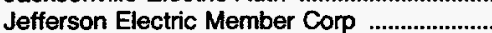

Jones-Onslow Elec Member Corp

Kinston City of

Lakeland City of ...........

Lamar Electric Membership Corp

Laurens Electric Coop Inc

Laurinburg City of ...

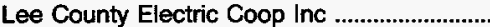

Leesburg City of

Lexington City of .........

Lumbee River Elec Member Corp ......................

Lumberton City of

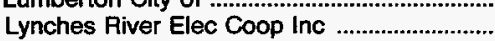

Manassas City of

Marietta City of

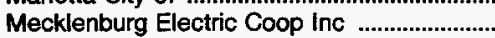

Mid-Carolina Electric Coop Inc



Monroe City of .

Morganton City of

See footnotes at end of table.

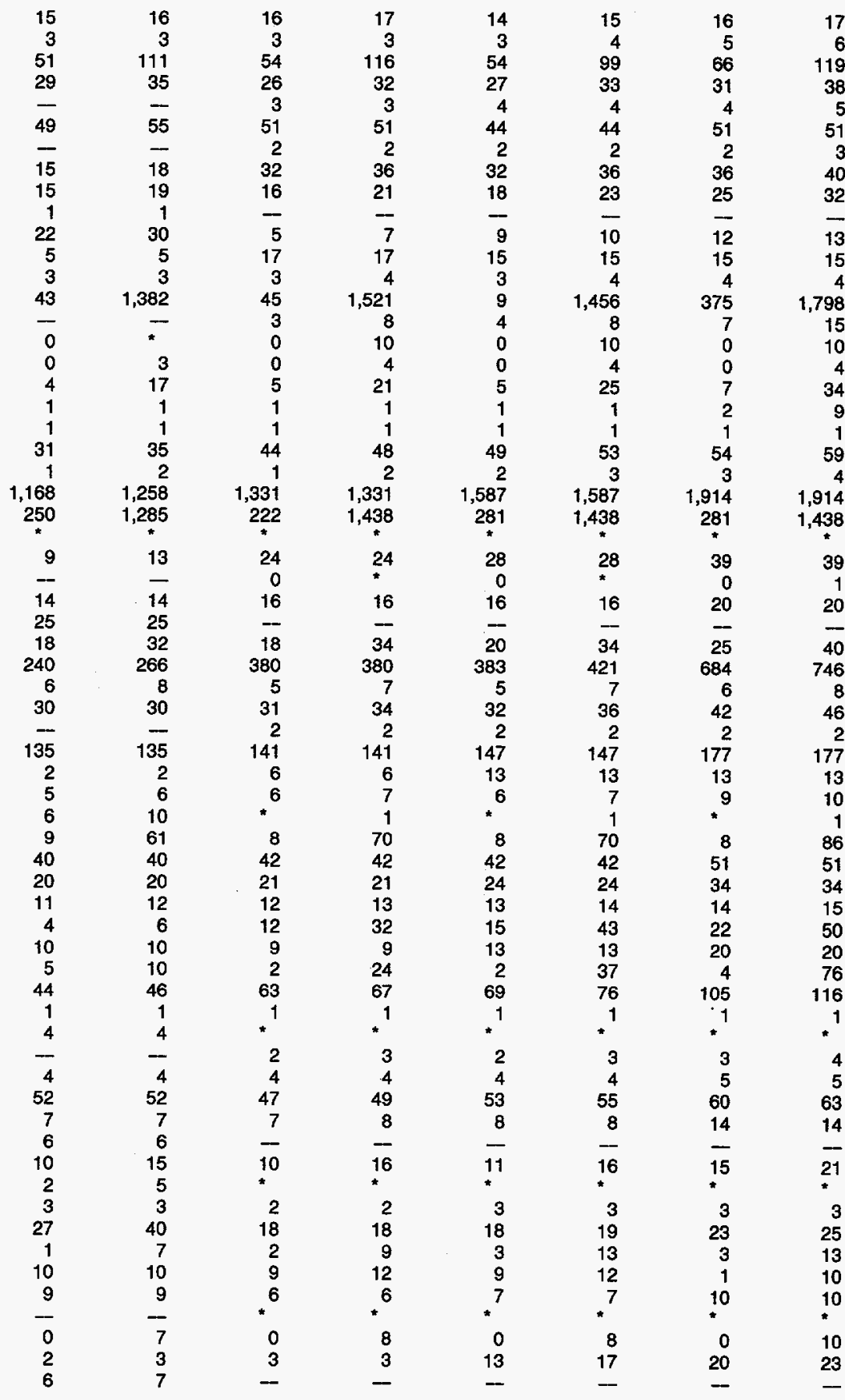


Table 18. U.S. Electric Utility Actual and Potential Peakload Reductions by North American Electric Reliability Council Region and Hawaii by Utility,

1992, 1993, 1994, and 1998

(Megawatts) (Continued)

\begin{tabular}{|c|c|c|c|c|c|c|c|c|}
\hline \multirow{3}{*}{$\begin{array}{c}\text { North American Electric Reliablity } \\
\text { Council Region and Hawall / } \\
\text { Electric Utility }\end{array}$} & \multicolumn{4}{|c|}{ Historical Reductions } & \multicolumn{4}{|c|}{ Projected Reductions } \\
\hline & \multicolumn{2}{|c|}{1992} & \multicolumn{2}{|c|}{1993} & \multicolumn{2}{|c|}{1994} & \multicolumn{2}{|c|}{1998} \\
\hline & Actual & Potential & Actual & Potential & Actual & Potential & Actual & Potential \\
\hline
\end{tabular}

SERC (Continued)

Municipal Electric Authority

New Bern City of

New River Light \& Power

New Smyrna Beach Utils Comm .......................

Newberry City of

North Carolina Mun Power Agny

Northern Virginia Elec Coop

North Carolina El Member Corp

North Carolina Eastern M P A

Northem Neck Elec Coop Inc

Ocala City of

Orangeburg City of .

Palmetto Electric Coop Inc

Orlando Utilities Comm .

Planters Electric Member Corp

Pee Dee Electric Member Corp

Piedmont Electric Member Corp

Prince George Electric Coop

Pulaski City of

Randolph Electric Member Corp

Rayle Electric Membership Corp

Reedy Creek Improvement Dist .

Roanoke Electric Member Corp

Rock Hill City of .

Rocky Mount City of .

Rutherford Elec

Satilla Rural Elec Member Corp

Sawnee Electric Members Corp

Seneca City of

Shelby City of

Shenandoah Valley Elec Coop

Singing River Elec Power Assn

South Carolina Pub Serv Auth

South Mississippi El Pwr Assn ...

Snapping Shoals El Member Corp

Statesville City of

Suwannee Valley Elec Coop Inc

Tallahassee City of

Tampa Electric Co

Tennessee Valley Authority

Thomasville City of

Tri-County Elec Member Corp

Tri-County Elec Member Corp

Tideland Electric Member Corp

Troup Electric Members Corp ...

Virginia Electric \& Power Co

Wake Electric Membership Corp

Walton Electric Member Corp

Washington City of

Washington Elec Member Corp

Withlacoochee River Elec Coop

York Electric Coop Inc

Southside Electric Coop Inc

Brunswick Electric Member Corp

Colquitt Electric Members Corp

Central Electric Pwr Coop Inc

Rappahannock Electric Coop

Alabama Municipal Elec Auth

SERC Total

\begin{tabular}{|c|c|}
\hline 0 & * \\
\hline 4 & 5 \\
\hline 1 & 3 \\
\hline 7 & 7 \\
\hline 2 & 2 \\
\hline 45 & 45 \\
\hline 35 & 37 \\
\hline 149 & 149 \\
\hline 68 & 68 \\
\hline 2 & 2 \\
\hline 6 & 7 \\
\hline 6 & 6 \\
\hline 8 & 12 \\
\hline 13 & 13 \\
\hline 4 & 6 \\
\hline 4 & 7 \\
\hline 13 & 13 \\
\hline 12 & 12 \\
\hline- & - \\
\hline 7 & 7 \\
\hline 2 & 3 \\
\hline- & - \\
\hline 3 & 14 \\
\hline- & - \\
\hline 14 & 19 \\
\hline 14 & 16 \\
\hline 8 & 14 \\
\hline 13 & 48 \\
\hline- & - \\
\hline 3 & 5 \\
\hline 6 & 6 \\
\hline 10 & 10 \\
\hline 100 & 100 \\
\hline 148 & 148 \\
\hline 32 & 32 \\
\hline 6 & 8 \\
\hline 34 & 34 \\
\hline 27 & 28 \\
\hline 0 & 1 \\
\hline 51 & 51 \\
\hline 240 & 696 \\
\hline 501 & 2,120 \\
\hline 5 & 5 \\
\hline 3 & 4 \\
\hline 4 & 4 \\
\hline 6 & 6 \\
\hline * & 6 \\
\hline 8 & 8 \\
\hline 186 & 245 \\
\hline 15 & 17 \\
\hline 21 & 27 \\
\hline 5 & 5 \\
\hline 3 & 4 \\
\hline 32 & 32 \\
\hline 13 & 18 \\
\hline 17 & 17 \\
\hline 15 & 20 \\
\hline 22 & 27 \\
\hline 76 & 81 \\
\hline 91 & 119 \\
\hline 6 & 6 \\
\hline 559 & 11,405 \\
\hline
\end{tabular}

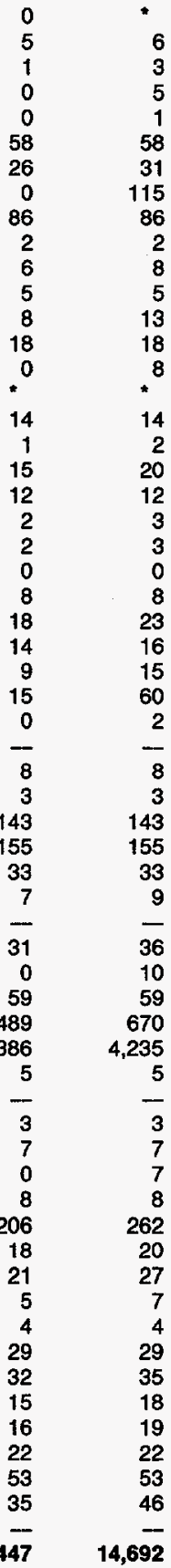

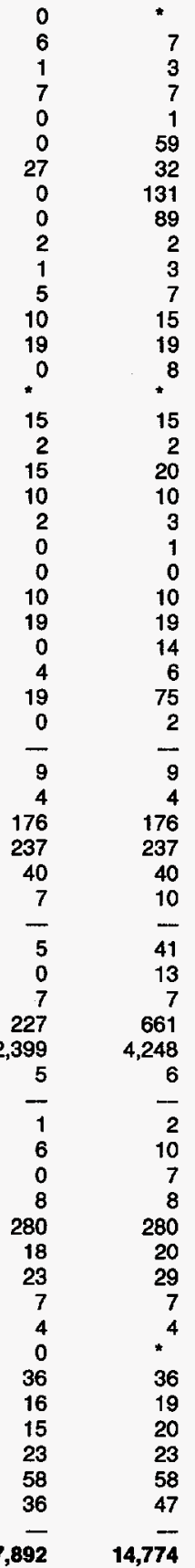

67

194

98

5

8

22

8

17

2

20
19

3

1 
Table 18. U.S. Electric Utility Actual and Potential Peakload Reductions by North American Electric Reliability Council Region and Hawaii by Utility,

1992, 1993, 1994, and 1998

(Megawatts) (Continued)

\begin{tabular}{|c|c|c|c|c|c|c|c|c|}
\hline \multirow{3}{*}{$\begin{array}{c}\text { North American Electric Reliability } \\
\text { Councll Reglon and Hawall / } \\
\text { Electric Utility }\end{array}$} & \multicolumn{4}{|c|}{ Historical Reductions } & \multicolumn{4}{|c|}{ Projected Reductions } \\
\hline & \multicolumn{2}{|c|}{1992} & \multicolumn{2}{|c|}{1993} & \multicolumn{2}{|c|}{1994} & \multicolumn{2}{|c|}{1998} \\
\hline & Actual & Potential & Actual & Potential & Actual & Potential & Actual & Potential \\
\hline \multicolumn{9}{|l|}{ SPP } \\
\hline Altus City of & 1 & 1 & - & - & - & - & - & - \\
\hline 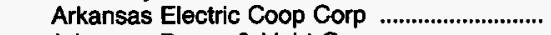 & - & - & 0 & 444 & 0 & 529 & 0 & 529 \\
\hline 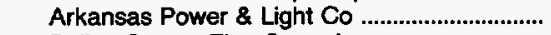 & 198 & 209 & 166 & 205 & 162 & 200 & 184 & 222 \\
\hline 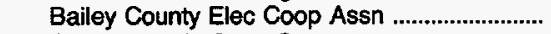 & - & - & 7 & 35 & 7 & 35 & 7 & 35 \\
\hline 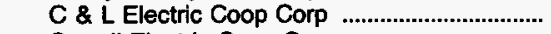 & 0 & 10 & 3 & 10 & 5 & 10 & 5 & 10 \\
\hline 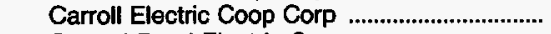 & 1 & 8 & 8 & 68 & 9 & 71 & 10 & 85 \\
\hline Central Rural Electric Coop ...................................... & 4 & 7 & 4 & 5 & 4 & 4 & 5 & 5 \\
\hline 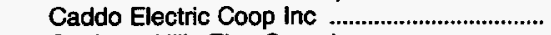 & 3 & 13 & 10 & 14 & 10 & 14 & 10 & 14 \\
\hline 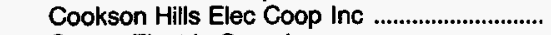 & 5 & 22 & 6 & 23 & 6 & 23 & 8 & 29 \\
\hline 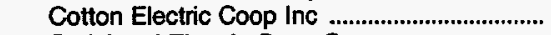 & 0 & * & 0 & 7 & 0 & 7 & 0 & 7 \\
\hline 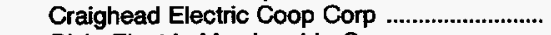 & 7 & 23 & 6 & 25 & 7 & 25 & 8 & 29 \\
\hline 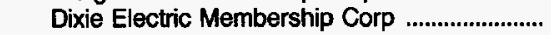 & 13 & 15 & - & - & - & - & - & - \\
\hline Duncan City of & 1 & 1 & * & * & * & * & * & * \\
\hline 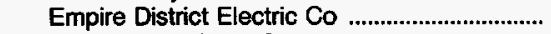 & 0 & 21 & 25 & 25 & 26 & 26 & 26 & 26 \\
\hline  & 15 & 32 & 20 & 46 & 20 & 46 & 24 & 59 \\
\hline Golden Spread Elec Coop Inc ............................ & 0 & 44 & 0 & 44 & 0 & 44 & 0 & 44 \\
\hline Gulf States Utilities Co & 1 & 125 & 1 & 126 & 1 & 128 & 2 & 210 \\
\hline 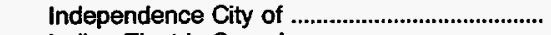 & 1 & 3 & 2 & 4 & 4 & 4 & 6 & 7 \\
\hline 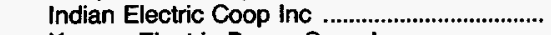 & 3 & 8 & 3 & 8 & 3 & 8 & 4 & 10 \\
\hline Kansas Electric Power Coop Inc ........................ & * & 15 & 8 & 30 & 0 & 23 & 0 & 29 \\
\hline Kansas City Power \& Light Co .............................. & 6 & 17 & 19 & 22 & 22 & 22 & 17 & 17 \\
\hline 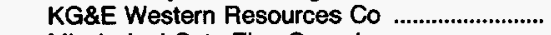 & 15 & 160 & 15 & 147 & 15 & 147 & 15 & 170 \\
\hline Mississippi Cnty Elec Coap Inc .......................... & 1 & 2 & 1 & 311 & 2 & 364 & 162 & 447 \\
\hline North Arkansas Elec Coop Inc .......................... & 5 & 5 & 5 & 5 & 5 & 5 & 5 & 5 \\
\hline Northeast Louisiana Power Coop ..................... & - & - & 3 & 3 & 0 & 0 & 0 & 0 \\
\hline 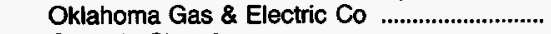 & 225 & 425 & 239 & 439 & 245 & 445 & 285 & 602 \\
\hline Osceola City of & 3 & 3 & 3 & 3 & 3 & 3 & 5 & 5 \\
\hline 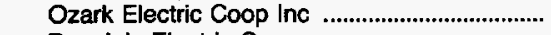 & 2 & 2 & 2 & 2 & 2 & 2 & 2 & 2 \\
\hline People's Electric Coop & 3 & 7 & - & - & -- & - & - & - \\
\hline Petit Jean Electric Coop Corp ............................ & 3 & 3 & 3 & 3 & 2 & 2 & 2 & 2 \\
\hline 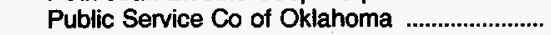 & 0 & 84 & 116 & 116 & 112 & 112 & 155 & 155 \\
\hline Red River Valley Rrl Elec Assn .............................. & 5 & 7 & $\mathbf{5}$ & 8 & 5 & 8 & 6 & 9 \\
\hline South Central Ark El Coop Inc ............................... & -- & - & 4 & 4 & 6 & 7 & 7 & 8 \\
\hline 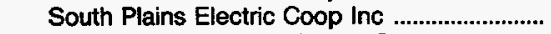 & 10 & 23 & 10 & 24 & 12 & 24 & 23 & 41 \\
\hline Southwestern Electric Power Co ....................... & 50 & 69 & 90 & 90 & 93 & 93 & 146 & 146 \\
\hline Southwestern Public Service Co ...................... & 3 & 279 & 24 & 242 & 27 & 295 & 44 & 534 \\
\hline 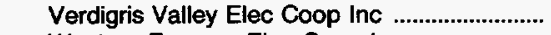 & 11 & 14 & 11 & 14 & 11 & 14 & 11 & 14 \\
\hline 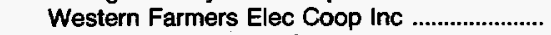 & 0 & 52 & 0 & 50 & 0 & 50 & 0 & 50 \\
\hline White River Valley El Coop Inc ............................. & - & - & 7 & 15 & 12 & 15 & 14 & 16 \\
\hline 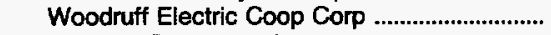 & 25 & 43 & 33 & 54 & 33 & 55 & 36 & 59 \\
\hline Western Resources inc ............................................. & 3 & 173 & 30 & 177 & 30 & 177 & 74 & 221 \\
\hline SPP Total & 624 & 1,925 & 889 & 2,846 & 901 & 3,034 & 1,309 & 3,850 \\
\hline \multicolumn{9}{|l|}{ wscc(U.s.) } \\
\hline 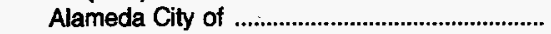 & 1 & 1 & 1 & 2 & 2 & 2 & 2 & 2 \\
\hline 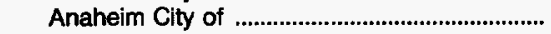 & 2 & 19 & 5 & 22 & 10 & 30 & 25 & 56 \\
\hline 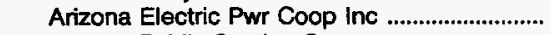 & 0 & 0 & 1 & 1 & 3 & 3 & 10 & 10 \\
\hline 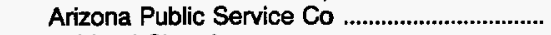 & 441 & 580 & 580 & 589 & 611 & 621 & 680 & 695 \\
\hline Ashland City of & & & 0 & 0 & 0 & 0 & 0 & 0 \\
\hline PUD No 1 of Benton County & 15 & 15 & - & - & - & - & - & - \\
\hline 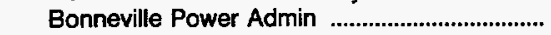 & 205 & 574 & 95 & 177 & 70 & 228 & 648 & 843 \\
\hline Bountiful City City of & 7 & 7 & 7 & 7 & 7 & 7 & 1 & 7 \\
\hline 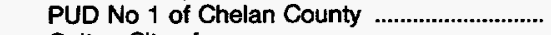 & 27 & 27 & 20 & 20 & 32 & 32 & 32 & 32 \\
\hline Colton City of & * & * & * & * & * & * & * & * \\
\hline 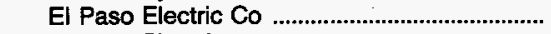 & 24 & 24 & 44 & 44 & 69 & 69 & 94 & 94 \\
\hline Eugene City of & 29 & 29 & - & -- & - & - & - & - \\
\hline Fort Collins City of & 5 & 16 & 5 & 16 & 5 & 16 & 15 & 26 \\
\hline Idaho Power Co & 25 & 25 & 26 & 26. & 43 & 43 & 115 & 115 \\
\hline 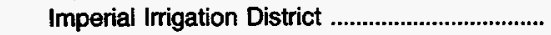 & 3 & 8 & 8 & 8 & 18 & 18 & 20 & 20 \\
\hline La Plata Electric Assn Inc ....................................... & 0 & 2 & 0 & 3 & 0 & 1 & 0 & 33 \\
\hline 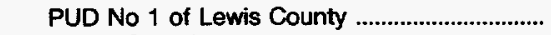 & $*$ & * & - & -- & - & - & - & - \\
\hline 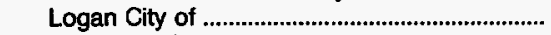 & 3 & 4 & - & - & - & - & - & - \\
\hline Longmont City of & 4 & 6 & 4 & 7 & 2 & 3 & 3 & 4 \\
\hline 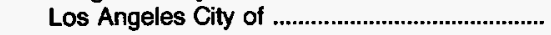 & 25 & 101 & 71 & 83 & 78 & 90 & 122 & 198 \\
\hline
\end{tabular}

See footnotes at end of table. 
Table 18. U.S. Electric Utility Actual and Potential Peakload Reductions by North American Electric Reliability Council Region and Hawail by Utility, 1992, 1993, 1994, and 1998

(Megawatts) (Continued)

\begin{tabular}{|c|c|c|c|c|c|c|c|c|}
\hline \multirow{3}{*}{$\begin{array}{c}\text { North American Electric Rellability } \\
\text { Council Reglon and Hawali / } \\
\text { Electric Utillty }\end{array}$} & \multicolumn{4}{|c|}{ Historical Reductions } & \multicolumn{4}{|c|}{ Projected Reductions } \\
\hline & \multicolumn{2}{|c|}{1992} & \multicolumn{2}{|c|}{1993} & \multicolumn{2}{|c|}{1994} & \multicolumn{2}{|c|}{1998} \\
\hline & Actual & Potential & Actual & Potential & Actual & Potential & Actual & Potential \\
\hline \multicolumn{9}{|l|}{ WSCC(U.S.) (Continued) } \\
\hline 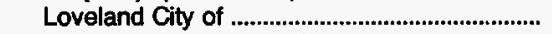 & 2 & 8 & 2 & 8 & 2 & 2 & 2 & 2 \\
\hline 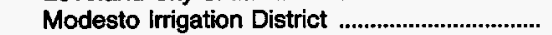 & 8 & 13 & 16 & 16 & 14 & $1 \overline{4}$ & 22 & 22 \\
\hline 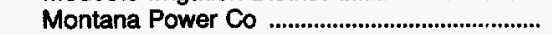 & 13 & 77 & 26 & 94 & 47 & 115 & 123 & 123 \\
\hline Navopache Electric Coop Inc .................................. & 5 & 15 & 8 & 15 & 8 & 15 & 9 & 17 \\
\hline 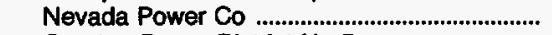 & 116 & 250 & 80 & 154 & 114 & 213 & 154 & 269 \\
\hline Overton Power District No $5 \ldots$ & -- & - & 1 & 1 & 1 & 1 & 2 & 2 \\
\hline Pacific Gas \& Electric Co ......................................... & 789 & 865 & 837 & 909 & 1,183 & 1,255 & 1,156 & 1,229 \\
\hline 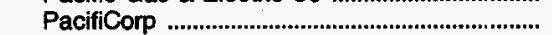 & 0 & 300 & 0 & 300 & 0 & $371^{\circ}$ & 0 & 371 \\
\hline 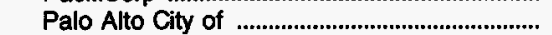 & 6 & 9 & 6 & 9 & 7 & 9 & 9 & 11 \\
\hline 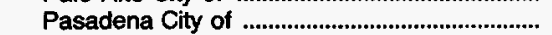 & 2 & 2 & 3 & 3 & 6 & 6 & 18 & 18 \\
\hline PUD No 2 of Grant County ................................. & 3 & 3 & - & - & - & - & - & - \\
\hline 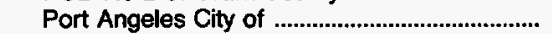 & * & $*$ & - & - & - & - & - & - \\
\hline Portland General Electric Co ................................. & 0 & 0 & 0 & 0 & 0 & 0 & 36 & 36 \\
\hline  & 145 & 191 & 122 & 251 & 206 & 254 & 372 & 430 \\
\hline 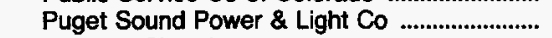 & 0 & 13 & 0 & 41 & 0 & 46 & 0 & 51 \\
\hline 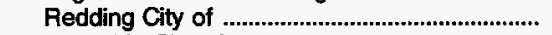 & 4 & 6 & 26 & 26 & 27 & 27 & 38 & 38 \\
\hline 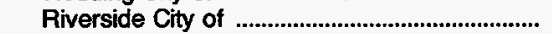 & 5 & 6 & 6 & 7 & 6 & 7 & 7 & 8 \\
\hline 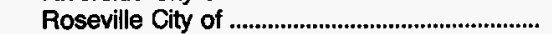 & 1 & 6 & 2 & 8 & 2 & 8 & 3 & 10 \\
\hline  & 281 & 281 & 309 & 309 & 353 & 353 & 548 & 548 \\
\hline 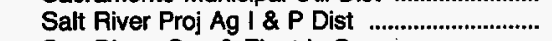 & 92 & 158 & 112 & 188 & 7 & 82 & 76 & 160 \\
\hline 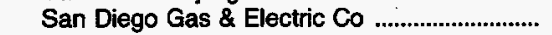 & 68 & 68 & 57 & 57 & 29 & 29 & 23 & 23 \\
\hline 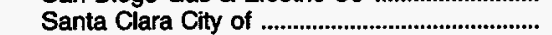 & 8 & 8 & 8 & 8 & 8 & 8 & 8 & 8 \\
\hline 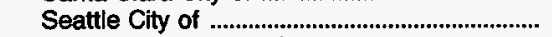 & 0 & 0 & 38 & 38 & 49 & 49 & 81 & 81 \\
\hline Sierra Pacific Power Co .......................................... & 40 & 40 & 31 & 31 & 38 & 38 & 84 & 84 \\
\hline 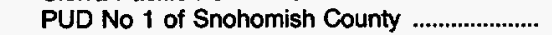 & 48 & 48 & - & - & - & - & - & - \\
\hline Southern California Edison Co .............................. & 1,316 & 2,695 & 1,487 & 3,020 & 1,603 & 3,209 & 2,035 & 3,904 \\
\hline Springfield City of & $\star$ & * & 1 & 1 & $*$ & : & 2 & 2 \\
\hline 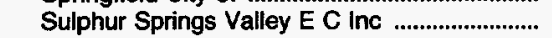 & 3 & 3 & 3 & 3 & 2 & 2 & 4 & 4 \\
\hline Tacoma City of & 31 & 31 & 0 & 0 & 0 & 0 & 0 & 0 \\
\hline 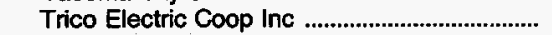 & - & - & 1 & 3 & 0 & 0 & 0 & 0 \\
\hline 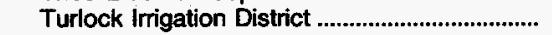 & 5 & 5 & 7 & 7 & 6 & 6 & 12 & 12 \\
\hline 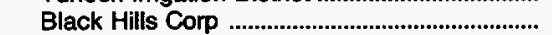 & 10 & 17 & 12 & 17 & 13 & 18 & 17 & 24 \\
\hline 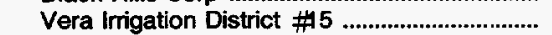 & 7 & 8 & 7 & 8 & * & * & $*$ & $\neq$ \\
\hline 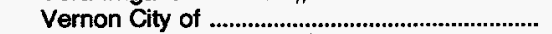 & 7 & 13 & 8 & 14 & 9 & 12 & 9 & 12 \\
\hline Washington Water Power Co .............................. & 48 & 48 & 103 & 103 & 136 & 136 & 184 & 184 \\
\hline 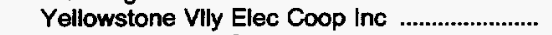 & • & * & 4 & 4 & 5 & 5 & 9 & 9 \\
\hline 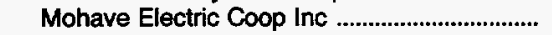 & 5 & 10 & 1 & 1 & 1 & 1 & 1 & 1 \\
\hline Tucson Electric Power Co ......................................... & 6 & 6 & 20 & 20 & 25 & 25 & 58 & 58 \\
\hline Northern California Power Agny ............................ & 11 & 12 & - & - & - & - & - & - \\
\hline $\begin{array}{l}\text { WSCC(U.S.) Total } \\
\text { Contiguous U.S. }\end{array}$ & $\begin{array}{r}3,902 \\
17,194\end{array}$ & $\begin{array}{r}6,653 \\
32,419\end{array}$ & $\begin{array}{r}4,210 \\
23,057\end{array}$ & $\begin{array}{r}6,677 \\
39,483\end{array}$ & $\begin{array}{r}4,856 \\
25,253\end{array}$ & $\begin{array}{r}7,479 \\
42,194\end{array}$ & $\begin{array}{r}6,868 \\
36,235\end{array}$ & $\begin{array}{r}9,886 \\
55,092\end{array}$ \\
\hline \multicolumn{9}{|l|}{ Ascc } \\
\hline Alaska Electric Light\&Power $\mathrm{Co} . . . . . . . . . . . . . . . . . . . . .$. & 6 & 6 & 7 & 7 & 5 & 5 & 5 & 5 \\
\hline  & * & * & * & * & 1 & 1 & 3 & 3 \\
\hline 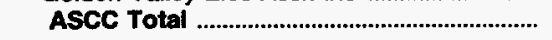 & 7 & 7 & 7 & 7 & 6 & 6 & 8 & 8 \\
\hline \multicolumn{9}{|l|}{ Hawail } \\
\hline Hawaii Electric Light Co Inc .................................. & - & - & * & * & 1 & 1 & 6 & 6 \\
\hline 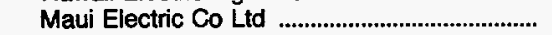 & 3 & 16 & 2 & 14 & 2 & 15 & 8 & 23 \\
\hline 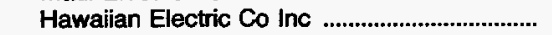 & 1 & 1 & 3 & 3 & 3 & 3 & 34 & 34 \\
\hline 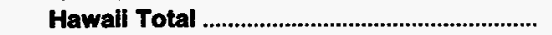 & & 17 & & 18 & & 19 & & \\
\hline U.S. Total & 17,204 & 32,442 & 23,069 & 39,508 & 25,266 & 42,220 & 36,291 & 55,163 \\
\hline
\end{tabular}

* Value less than 0.5 .

Notes: $\bullet$ Data are final. -Data are provided for electric utilities with sales to ultimate consumers or sales for resale greater than or equal to 120,000 megawatthours. - Totals may not equal sum of components because of independent rounding.

Source: Energy Information Administration, Form EIA-861, "Annual Electric Utility Report." 
Table 19. U.S. Electric Utility Actual Peakload Reductions by North American Electric Reliability Council Region and Hawaii by DSM Program Category, 1993 (Megawatts)

\begin{tabular}{|c|c|c|c|c|c|c|}
\hline $\begin{array}{c}\text { North American Electric Rellability } \\
\text { Councll Reglon and Hawail / } \\
\text { Electrlc Utility }\end{array}$ & $\begin{array}{c}\text { Energy } \\
\text { Efflolency }\end{array}$ & $\begin{array}{l}\text { Direct Load } \\
\text { Control }\end{array}$ & $\begin{array}{l}\text { Interruptible } \\
\text { Load }\end{array}$ & $\begin{array}{l}\text { Other Load } \\
\text { Management }\end{array}$ & $\begin{array}{c}\text { Other Demand- } \\
\text { Side Management }\end{array}$ & $\begin{array}{l}\text { Total DSM } \\
\text { Programs }\end{array}$ \\
\hline \multicolumn{7}{|l|}{ ECAR } \\
\hline Appalachian Power Co ...................... & 14 & 0 & 132 & 0 & 0 & 146 \\
\hline Cincinnati Gas \& Electric Co ............ & 6 & 0 & 17 & 0 & 0 & 22 \\
\hline Cleveland Electric fllum Co ............... & 4 & 0 & 0 & 1 & 0 & 6 \\
\hline Columbus Southern Power Co ......... & 2 & 0 & 20 & 3 & 2 & 27 \\
\hline Consumers Power Co ............................. & 110 & 1 & 20 & * & 4 & 135 \\
\hline Dayton Power \& Light Co ................... & 16 & 0 & 0 & 0 & 0 & 16 \\
\hline 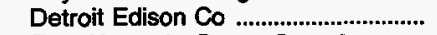 & 19 & 6 & 0 & 0 & 0 & 25 \\
\hline East Kentucky Power Coop Inc ........ & 9 & 0 & 0 & 2 & 0 & 11 \\
\hline 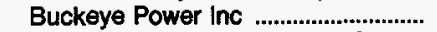 & 0 & 87 & 32 & 0 & 0 & 119 \\
\hline Hendricks County Rural E M C ........ & 0 & 4 & $*$ & 0 & 0 & 4 \\
\hline Indianapolis Power \& Light $\mathrm{Co}$ & * & 0 & $\mathbf{0}$ & 0 & 14 & 14 \\
\hline Indiana Michigan Power Co .............. & 1 & 0 & 29 & 7 & * & 37 \\
\hline 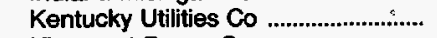 & 7 & 0 & 0 & 3 & 9 & 20 \\
\hline Kingsport Power Co ............................. & 1 & 0 & 0 & 0 & 0 & 1 \\
\hline Lansing City of & 0 & 0 & 0 & * & 0 & * \\
\hline Louisville Gas \& Electric Co ............... & 0 & 0 & 64 & 0 & 0 & 64 \\
\hline Monongahela Power Co ..................... & 68 & 0 & 0 & 8 & 0 & 76 \\
\hline 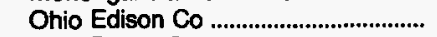 & 8 & 0 & 244 & 0 & 0 & 252 \\
\hline 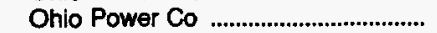 & 3 & 0 & 99 & 16 & 1 & 120 \\
\hline Owen Electric Coop Inc ....................... & 1 & 0 & 0 & 0 & 0 & 1 \\
\hline Pennsylvania Power Co .......................... & 0 & 0 & 40 & 0 & 0 & 40 \\
\hline 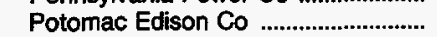 & 158 & 0 & 0 & 0 & 0 & 158 \\
\hline 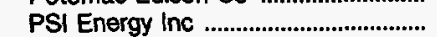 & 47 & 0 & 30 & 0 & 0 & 76 \\
\hline Southern Indiana Gas \& Elec Co .... & 4 & 9 & 0 & 0 & 0 & 13 \\
\hline South Central Power Co ..................... & 0 & 25 & 0 & 0 & 0 & 25 \\
\hline 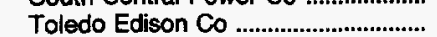 & 3 & 0 & 0 & 1 & 0 & 5 \\
\hline Utilities Dist-Western IN REMC ........ & 8 & 0 & 0 & 3 & 0 & 11 \\
\hline Virginia Tech Electric Service ........... & 0 & 0 & 0 & 2 & 0 & 2 \\
\hline 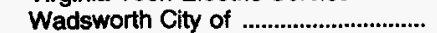 & 0 & 0 & 8 & $\overrightarrow{0}$ & 0 & 8 \\
\hline West Penn Power Co .......................... & 81 & 0 & 0 & 65 & 0 & 145 \\
\hline 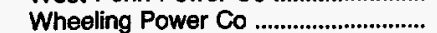 & 1 & 0 & 0 & 2 & 0 & 3 \\
\hline Wolverine Pwr Supply Coop Inc ....... & 0 & 8 & 0 & 0 & 0 & 8 \\
\hline Kentucky Power Co ........................... & 2 & 0 & 33 & 0 & 0 & 35 \\
\hline Wabash Valley Power Assn Inc ...... & $\overline{0}$ & 40 & 0 & 0 & 0 & 40 \\
\hline American Mun Power-Ohio Inc ......... & 0 & 0 & 5 & 1 & 0 & 6 \\
\hline 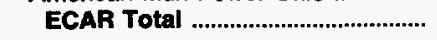 & 573 & 179 & 773 & 115 & 31 & 1,671 \\
\hline \multicolumn{7}{|l|}{ ERCOT } \\
\hline 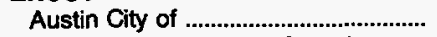 & 207 & 3 & 0 & 0 & 2 & 212 \\
\hline Brazos Electric Power Coop Inc ...... & 1 & 0 & 0 & 0 & 0 & 1 \\
\hline Bryan City of & 6 & 0 & 4 & 0 & 0 & 9 \\
\hline Central Power \& Light $\mathrm{Co}$ & 57 & 0 & 0 & 0 & 14 & 71 \\
\hline 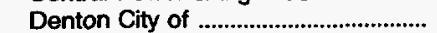 & * & 2 & 0 & 1 & 0 & 3 \\
\hline 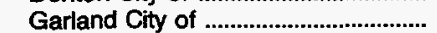 & 0 & 6 & 8 & 0 & 0 & 14 \\
\hline 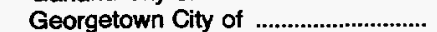 & * & 0 & 0 & 0 & 0 & * \\
\hline Greenville Electric Util Sys ................ & 0 & 0 & 2 & 0 & 1 & 3 \\
\hline Guadalupe Valley Elec Coop Inc .... & 0 & 5 & 50 & 6 & 0 & 61 \\
\hline Houston Lighting \& Power Co .......... & 48 & 0 & 0 & 0 & 0 & 48 \\
\hline Johnson County Elec Coop Assn ... & * & 0 & 0 & 2 & 0 & $\begin{array}{r}40 \\
2\end{array}$ \\
\hline Lower Colorado River Authority ....... & 72 & 20 & 0 & 0 & 0 & 92 \\
\hline Magic Valley Electric Coop Inc ......... & 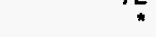 & 0 & 0 & 0 & 0 & $*$ \\
\hline Medina Electric Coop Inc .................. & 0 & 0 & 0 & 6 & 0 & 6 \\
\hline San Bernard Electric Coop Inc ......... & * & 6 & 4 & 4 & 0 & 14 \\
\hline Tri-County Electric Coop Inc .............. & 2 & 0 & 0 & 0 & 0 & 2 \\
\hline West Texas Utilities Co .......................... & 0 & 0 & 39 & 0 & 0 & 39 \\
\hline  & 3 & - & 0 & 0 & 0 & 3 \\
\hline Texas-New Mexico Power Co ........... & 11 & 0 & 8 & 0 & 0 & 19 \\
\hline Texas Utilities Electric Co .................. & 541 & 0 & 0 & 273 & 0 & 814 \\
\hline 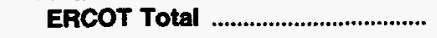 & 949 & 42 & 114 & 291 & 17 & 1,414 \\
\hline \multicolumn{7}{|l|}{ MAAC } \\
\hline 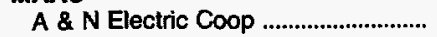 & 0 & 1 & 0 & 0 & 0 & 1 \\
\hline Atlantic City Electric Co ...................... & 24 & 30 & 22 & 18 & 0 & 94 \\
\hline 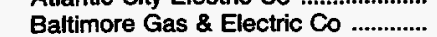 & 59 & 0 & 0 & 0 & 0 & 59 \\
\hline Choptank Electric Coop Inc .............. & 0 & 0 & 0 & 1 & 4 & 5 \\
\hline Conowingo Power Co & * & 3 & 0 & 0 & 0 & 3 \\
\hline Delmarva Power \& Light Co ............ & 12 & 101 & 45 & 4 & 0 & 162 \\
\hline Delaware Electric Coop Inc ............... & 0 & 6 & 0 & 0 & 0 & 6 \\
\hline 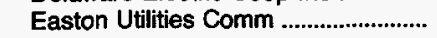 & & 0 & 0 & 0 & 0 & * \\
\hline
\end{tabular}

See footnotes at end of table. 
Table 19. U.S. Electric Utility Actual Peakload Reductions by North American Electric Reliability Council Region and Hawail by DSM Program Category, 1993 (Megawatts) (Continued)

\begin{tabular}{c|c|c|c|c|c|}
\hline $\begin{array}{c}\text { North American Electric Reliability } \\
\text { Council Region and Hawali / } \\
\text { Electrlc Utility }\end{array}$ & $\begin{array}{c}\text { Energy } \\
\text { Efficiency }\end{array}$ & $\begin{array}{c}\text { Direct Load } \\
\text { Control }\end{array}$ & $\begin{array}{c}\text { Interruptlble } \\
\text { Load }\end{array}$ & $\begin{array}{c}\text { Other Load } \\
\text { Management }\end{array}$ & $\begin{array}{c}\text { Other Demand- } \\
\text { Side Management }\end{array}$ \\
\hline
\end{tabular}

\section{MAAC}

Jersey Central Power\&Light Co .......

Metropolitan Edison Co

Pennsylvania Electric Co

Pennsylvania Power \& Light $\mathrm{Co}_{0} . . . .$.

Philadelphia Electric Co

Potomac Electric Power Co

Public Service Electric\&Gas Co .......

Southern Maryland El Coop Inc ......

Somerset Rural Elec Coop Inc ........

Adams Electric Coop Inc

Bedford Rural Elec Coop Inc ............

Valley Rural Electric Coop Inc ..........

Central Electric Coop Inc ...................

Claverack Rural Elec Coop Inc .......

Northwestern Rural E C A Inc

MAAC Total

$\begin{array}{rr}29 & 39 \\ 29 & 0 \\ 51 & 0 \\ 11 & 0 \\ 4 & 40 \\ 46 & 0 \\ 33 & 53 \\ 2 & 30 \\ 0 & 1 \\ * & 7 \\ 0 & 2 \\ 0 & 3 \\ 0 & 4 \\ 0 & 5 \\ 0 & 5 \\ 301 & 329\end{array}$

72
63
0
156
0
0
158
0
0
0
0
0
0
0
0
516

\section{MAIN}

Boone Electric Coop

Central Illinois Light Co ......................

Coles-Moultrie Electric Coop .............

Columbia City of

Commonwealth Edison $\mathrm{Co}$

Com Belt Electric CoO

Cuivre River Electric Coop Inc ........

Eastern Illini Electric Coop

Illinois Power $\mathrm{Co}$

Madison Gas \& Electric Co ...............

Marshfield City of

Shelby Electric Coop Inc

Southwestern Electric Coop Inc .......

Springfield City of

Tri-County Electric Coop Inc .............

Union Electric Co

Wisconsin Electric Power Co ..............

Wisconsin Power \& Light Co

Wisconsin Public Power Inc Sys .....

Wisconsin Public Service Corp ......

MAIN Total .

MAPP(U.S.)

Ames City of

Anoka City of

Austin City of .

Barron

Beatrice City of

Cedar Falls City of

Central lowa Power Coop

Central Power Elec Coop inc ..........

Clark Electric Coop

Coop Power Assn

Comhusker Public Power Dist

Dawson County Public Pwr Dist ......

East River Elec Power Coop Inc .....

Fairmont Public Utilities Comm .........

Freeborn-Mower Electric Coop .........

Grant-Lafayette Electric Coop ..........

IES Utilities Inc

Interstate Power Co

lowa Lakes Electric Coop ...................

L \& O Power Coop .......

Uncoln Electric System

Marshall City of

MDU Resources Group Inc

Nodak Electric Coop Inc

Midland Power Coop

Minnesota Power \& Light Co

0
0
5
0
0
0
0
0
0
0
0
0
0
0
0
7
70
25
1
0
0
0
0
0
0
0

$\begin{array}{rrr}0 & 0 & * \\ 0 & 0 & * \\ 0 & 0 & 5 \\ 0 & 0 & 6 \\ 0 & 0 & 4 \\ 0 & 0 & 5 \\ 0 & 0 & 5 \\ 0 & 0 & 15 \\ 0 & 0 & 4 \\ 0 & 0 & 2 \\ 0 & 0 & 6 \\ 0 & 0 & * \\ 0 & 0 & 43 \\ 0 & 0 & 3 \\ 0 & * & 5 \\ 0 & 0 & 178 \\ 96 & 0 & 38 \\ 0 & 0 & 2 \\ 2 & 0 & 1 \\ 0 & 3 & 3 \\ * & 0 & 7 \\ 0 & 0 & 6 \\ 0 & 0 & 16 \\ 0 & 0 & 0\end{array}$

See footnotes at end of table. 
Table 19. U.S. Electric Utility Actual Peakload Reductions by North American Electric Reliability Council Region and Hawaii by DSM Program Category, 1993

(Megawatts) (Continued)

\begin{tabular}{c|c|c|c|c|c|c|}
\hline $\begin{array}{c}\text { North American Electric Rellabllity } \\
\text { Council Region and Hawall / } \\
\text { Electric Utility }\end{array}$ & $\begin{array}{c}\text { Energy } \\
\text { Efficiency }\end{array}$ & $\begin{array}{c}\text { Direct Load } \\
\text { Control }\end{array}$ & $\begin{array}{c}\text { Interruptible } \\
\text { Load }\end{array}$ & $\begin{array}{c}\text { Other Load } \\
\text { Management }\end{array}$ & $\begin{array}{c}\text { Other Demand- } \\
\text { Side Management }\end{array}$ & $\begin{array}{c}\text { Total DSM } \\
\text { Programs } 1\end{array}$ \\
\hline
\end{tabular}

MAPP(U.S.)

Minnkota Power Coop Inc

Moorhead City of

Nebraska Public Power Distict

Norris Public Power District ...............

North Platte City of .

Northern States Power Co

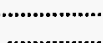

Northern States Power Co

Northwest lowa Power Coop ............

Northwestern Public Service Co ......

Oakdale Electric Coop

Oliver-Mercer Elec Coop Inc .............

Omaha Public Power District ............

Otter Tail Power Co

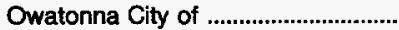

People's Coop Power Assn

Pella City of

Pierre City

Polk-Burr

Rochester Public Utilities

Roseau Electric Coop Inc

Runestone Electric Assn

Shakopee Public Utilities Comm ....

Superior Water Light\&Power Co ......

Tri-County Electric Coop .....................

United Power Assn

Verendrye Electric Coop Inc ..............

Vernon Electric Coop

Mountrail-Williams El CoOp Inc

Wild Rice Electric Coop Inc

Municipal Energy Agency of NE ......

Midwest Power Systems inc

Cass County Electric Coop Inc ........

MAPP(U.S.) Total

(n...............

\begin{tabular}{rr}
0 & 285 \\
$*$ & 10 \\
0 & 2 \\
0 & 1 \\
90 & 4 \\
226 & 19 \\
7 & 68 \\
0 & 0 \\
$*$ & 0 \\
0 & 4 \\
2 & 2 \\
7 & 0 \\
0 & 0 \\
$*$ & 4 \\
0 & 4 \\
1 & $*$ \\
0 & 4 \\
$*$ & 8 \\
0 & 2 \\
$*$ & 19 \\
0 & 3 \\
$*$ & 0 \\
$*$ & 0 \\
$*$ & 9 \\
2 & 46 \\
$*$ & 2 \\
3 & 7 \\
0 & 2 \\
5 & 19 \\
30 & 14 \\
$*$ & 25 \\
\hline & 52 \\
& 793
\end{tabular}

0
2
0
0
2
11
355
0
0
0
0
0
5
0
0
.
0
5
0
2
1
0
0
0
0
0
0
0
121
6
632

$\begin{array}{rr}0 & 0 \\ 0 & * \\ 4 & 0 \\ 0 & 0 \\ 1 & 0 \\ 7 & 0 \\ 43 & 0 \\ 0 & 0 \\ 0 & 0 \\ 0 & 0 \\ 0 & 0 \\ 0 & 0 \\ 56 & 5 \\ 0 & 0 \\ 0 & 0 \\ 0 & 0 \\ 0 & 0 \\ 0 & 0 \\ 0 & * \\ 0 & 0 \\ 0 & * \\ 0 & 0 \\ * & * \\ 0 & 0 \\ 60 & 0 \\ 0 & 0 \\ 0 & 0 \\ 0 & 0 \\ 0 & 0 \\ 1 & 0 \\ 0 & 0 \\ 0 & 0 \\ 270 & 0 \\ & 0 \\ 0 & 0 \\ 0 & 0 \\ 0 & 0 \\ 0 & 0 \\ 0 & 0\end{array}$

285

12

7

126

691

NPCC(U.S.)

Bangor Hydro-Electric Co

Blackstone Valley Electric Co .........

Boston Edison Co

Braintree Town of

Burlington City of

Cambridge Electric Light $\mathrm{Co}$

Central Hudson Gas \& Elec Carp .

Central Maine Power Co

Central Vermont Pub Serv Corp ......

Chicopee City of

Commonwealth Electric $\mathrm{Co}$...................

Concord Electric $\mathrm{Co}_{0}$

Connecticut Light \& Power Co ........

Connecticut Valley Elec Co Inc .......

Consolidated Edison Co-NY Inc ......

Eastern Edison Co

Exeter \& Hampton Electric Co .........

Fitchburg Gas \& Elec Light Co .......

Green Mountain Power Corp ...........

Holyoke City of

Hingham City of

Jamestown City of ...

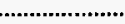

Maine Public Service Co ....................

Massachusetts Electric Co ...............

Massena Town of

Montaup Electric Co

Narragansett Electric Co ..................

New Hampshire Elec Coop Inc .......

New York State Elec \& Gas Corp

Newport Electric Corp

$\begin{array}{rr}7 & 1 \\ 11 & 0 \\ 88 & 0 \\ * & 1 \\ 3 & 0 \\ 5 & 0 \\ 23 & 0 \\ 67 & 17 \\ 10 & 110 \\ 1 & 0 \\ * & * \\ 14 & 0 \\ 1 & 0 \\ 235 & 18 \\ * & 0 \\ 145 & 0 \\ 19 & 0 \\ 1 & 0 \\ 2 & 0 \\ 8 & 7 \\ 1 & 0 \\ * & 2 \\ * & 0 \\ 131 & 0 \\ 1 & 0 \\ 142 & 5 \\ * & 1 \\ 32 & 0 \\ 54 & 2 \\ 2 & 0 \\ 155 & 0 \\ 2 & \end{array}$

\begin{tabular}{|c|c|c|c|}
\hline 0 & 0 & 0 & 7 \\
\hline 0 & 1 & 0 & 12 \\
\hline 13 & 0 & 0 & 101 \\
\hline 2 & 0 & 0 & 3 \\
\hline 0 & 0 & 0 & 3 \\
\hline 1 & 0 & 0 & 6 \\
\hline 0 & $*$ & 0 & 23 \\
\hline 0 & 0 & 0 & 84 \\
\hline 0 & 2 & 0 & 122 \\
\hline 0 & 0 & 0 & 1 \\
\hline 7 & 0 & 0 & 7 \\
\hline 6 & 0 & 0 & 19 \\
\hline 0 & 0 & 0 & 1 \\
\hline 14 & 0 & 0 & 267 \\
\hline 0 & 5 & 0 & 5 \\
\hline 39 & 0 & 0 & 184 \\
\hline 0 & 7 & 0 & 25 \\
\hline 0 & 0 & 0 & 1 \\
\hline 0 & 0 & 0 & 2 \\
\hline 0 & 0 & 0 & 15 \\
\hline 0 & 0 & * & 1 \\
\hline 1 & 0 & $*$ & 4 \\
\hline 0 & 0 & 0 & * \\
\hline 64 & 0 & 0 & 194 \\
\hline 0 & 0 & 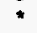 & 1 \\
\hline 0 & 0 & 0 & 147 \\
\hline 0 & 0 & 0 & 1 \\
\hline 0 & 0 & 0 & 32 \\
\hline 0 & 0 & 0 & 56 \\
\hline 13 & * & 0 & 31 \\
\hline 3 & 0 & 0 & 157 \\
\hline 0 & 0 & 0 & 2 \\
\hline
\end{tabular}

See footnotes at end of table. 
Table 19. U.S. Electric Utility Actual Peakload Reductions by North American Electric Reliability Council Region and Hawaii by DSM Program Category, 1993 (Megawatts) (Continued)

\begin{tabular}{|c|c|c|c|c|c|c|}
\hline $\begin{array}{c}\text { North American Electric Reliability } \\
\text { Council Region and Hawall / } \\
\text { Electric Utility }\end{array}$ & $\begin{array}{l}\text { Energy } \\
\text { Efficiency }\end{array}$ & $\begin{array}{l}\text { Direct Load } \\
\text { Control }\end{array}$ & $\begin{array}{l}\text { Interruptible } \\
\text { Load }\end{array}$ & $\begin{array}{l}\text { Other Load } \\
\text { Management }\end{array}$ & $\begin{array}{c}\text { Other Demand- } \\
\text { Side Management }\end{array}$ & $\begin{array}{c}\text { Total DSM } \\
\text { Programs }\end{array}$ \\
\hline
\end{tabular}

NPCC(U.S.)

Niagara Mohawk Power Corp ........... Norwood City of

Orange \& Rockland Utils Inc

Power Authority of State of NY .......

Public Service Co of NH

Reading Town of

Rochester Gas \& Electric Corp

Shrewsbury Town of

Taunton City of

United llluminating Co

Western Massachusetts Elec Co ... Granite State Electric Co

NPCC(U.S.) Total
SERC

Aiken Electric Coop Inc

Alabama Electric Coop Inc ................. Alabama Power Co

Albemarle City of

Altamaha Electric Member Corp Amicalola Electric Member Corp ..... Athens City of

BARC Electric Coop Inc

Berkeley Electric Coop Inc

Black River Electric Coop Inc ........... Bristol City of

Canoochee Electric Member Corp . Carolina Power \& Light Co ..

Carroll Electric Member Corp ............ Carteret-Craven El Member Corp .... Central Florida Elec Coop Inc .......... Central Georgia El Member Corp .... Central Electric Member Corp ..........

Clay Electric Coop Inc

Coast Electric Power Assn ................. Coastal Electric Member Corp ........... Cobb Electric Membership Corp ....

Community Electric Coop

Coweta-Fayette El Member Corp ... Crescent Electric Member Corp ...... Davidson Electric Member Corp ...... Dothan City of

Douglas City of

Duke Power Co

East Point City of Fayetteville Public Works Comm ..... Fitzgerald Wtr Lgt \& Bond Comm .. Flint Electric Membership Corp ....... Florida Keys El Coop Assn Inc ....... Florida Power \& Light Co .

Florida Power Corp

Fort Pierce Utilities Auth ......................

Four County Elec Member Corp ....... Gainesville Regional Utilities

GreyStone Power Corp

Georgia Grady County Elec Member Corp ... Greenville Utilities Comm

Griffin City of ...

Gulf Power Co .....

Harrisonburg City of

Hart Electric Member Corp

Haywood Electric Member Corp ......

High Point Town of

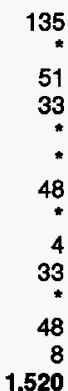

1,520

135

$\begin{array}{rr}0 & 0 \\ 1 & \\ 0 & 47 \\ 0 & 0 \\ 0 & 0 \\ 0 & 6 \\ 1 & 0 \\ 0 & 0 \\ 8 & 0 \\ 0 & 13 \\ 11 & 0 \\ 201 & 0 \\ 201 & 228\end{array}$

$\begin{array}{rr}0 & 0 \\ 47 & 0 \\ 0 & 0 \\ 0 & 0 \\ 6 & 0 \\ 0 & 0 \\ 0 & 1 \\ 0 & 1 \\ 13 & 0 \\ 0 & 1 \\ 0 & 0 \\ 0 & 0 \\ 228 & 0 \\ & 18\end{array}$

135
1
98
33
$*$
6
49
3
4
57
59
8
1,968

0

$\begin{aligned} 0 & \\ 0 & \\ 0 & \\ * & \\ 0 & \\ 0 & \\ 0 & \\ 0 & \\ 0 & \\ 12 & \\ 0 & \\ 100 & \end{aligned}$

$\begin{array}{rl}0 & 1 \\ 0 & 1 \\ 16 & 0 \\ 0 & 0\end{array}$

4
6
84
$*$

0

2

0

0 
Table 19. U.S. Electric Utility Actual Peakload Reductions by North American Electric Reliability Council Region and Hawaii by DSM Program Category, 1993 (Megawatts) (Continued)

\begin{tabular}{|c|c|c|c|c|c|c|}
\hline $\begin{array}{c}\text { North American Electric Rellability } \\
\text { Council Region and Hawail / } \\
\text { Electric Utillty }\end{array}$ & $\begin{array}{c}\text { Energy } \\
\text { Efficiency }\end{array}$ & $\begin{array}{l}\text { Direct Load } \\
\text { Control }\end{array}$ & $\begin{array}{l}\text { Interruptible } \\
\text { Load }\end{array}$ & $\begin{array}{l}\text { Other Load } \\
\text { Management }\end{array}$ & $\begin{array}{l}\text { Other Demand- } \\
\text { Side Management }\end{array}$ & $\begin{array}{c}\text { Total DSM } \\
\text { Programs }\end{array}$ \\
\hline
\end{tabular}

SERC

Jackson Electric Member Corp .......

Jacksonville Electric Auth

Jefferson Electric Member Corp .....

Jones-Onslow Elec Member Corp.

Kinston City of

Kissimmee Utility Authority

Lakeland City of

Lamar Electric Membership Corp ...

Laurens Electric Coop Inc

Laurinburg City of

Lawrenceville City of

Lee County Electric Coop Inc ...........

Leesburg City of

Lumbee River Elec Member Corp

Lumberton City of

Lynches River Elec Coop Inc .........

Manassas City of

Marietta City of .....................................

Mecklenburg Electric Coop Inc .......

Mid-Carolina Electric Coop Inc ........

Mississippi Power Co

Monroe City of

New Bern City of

New River Light \& Power Co

North Carolina Mun Power Agny .....

Northern Virginia Elec Coop

North Carolina Eastern M P A .........

Northern Neck Elec Coop Inc

Ocala Gity of

Orangeburg City of

Pee Dee Electrio Member Corp .....

Piedmont Electric Member Corp .....

Prince George Electric Coop

Pulaski City of

Randolph Electric Member Corp....

Rayle Electric Membership Corp .....

Reedy Creek Improvement Dist ......

Rock Hill City of

Rocky Mount City of ..........................

Rutherford Elec Member Corp ..........

Satilla Rural Elec Member Corp .....

Sawnee Electric Members Corp ......

Shenandoah Valley Elec Coop.

Singing River Elec Power Assn .......

South Carolina Flectric\&Gas Co

South Carolina Pub Serv Auth .........

South Mississippi El Pwr Assn .........

Snapping Shoals El Member Corp .

Sumter Electric Coop Inc ...................

Tallahassee City of ................................

Tampa Electric Co

Tennessee Valley Authority ..............

Thomasville City of

Tri-County Elec Member Corp ..........

Tideland Electric Member Corp .......

Union Electric Membership Corp ....

Virginia Electric \& Power Co ............

Wake Electric Membership Corp ....

Walton Electric Member Corp ..........

Washington City of

Washington Elec Member Corp ......

Withlacoochee River Elec Coop ......

York Electric Coop Inc

Southside Electric Coop Inc

Brunswick Electric Member Corp ... 
Table 19. U.S. Electric Utility Actual Peakload Reductions by North American Electric Reliability Council Region and Hawaii by DSM Program Category, 1993 (Megawatts) (Continued)

\begin{tabular}{c|c|c|c|c|c|}
\hline $\begin{array}{c}\text { North American Electric Reliability } \\
\text { Council Region and Hawall / } \\
\text { Electric Utility }\end{array}$ & $\begin{array}{c}\text { Energy } \\
\text { Efficlency }\end{array}$ & $\begin{array}{c}\text { Direct Load } \\
\text { Control }\end{array}$ & $\begin{array}{c}\text { Interruptible } \\
\text { Load }\end{array}$ & $\begin{array}{c}\text { Other Load } \\
\text { Management }\end{array}$ & $\begin{array}{c}\text { Other Demand- } \\
\text { Slde Management }\end{array}$ \\
\hline
\end{tabular}

SERC

Colquitt Electric Members Corp ....... Central Electric Pwr Coop Inc

Rappahannock Electric Coop ...........

SERC Total

0
0
0
$\mathbf{3 , 3 2 9}$

22
37
20
1,770

SPP

Arkansas Power \& Light Co

C \& L Electric Coop Corp

Carroll Electric Coop Corp

Central Rural Electric Coop

Caddo Electric Coop Inc ....................

Cookson Hills Elec Coop Inc .............

Craighead Electric Coop Corp .........

Duncan City of

Empire District Electric Co .................

First Electric Coop Corp .

Gulf States Utilities Co

Independence City of

Indian Electric Coop Inc ....................

Kansas Electric Power Coop Inc ....

Kansas City Power \& Light Co ........

Kansas Gas \& Electric Co

Mississippi Cnty Elec Coop Inc ........

North Arkansas Elec Coop Inc .......

Northeast Louisiana Power Coop ...

Oklahoma Gas \& Electric Co ............

Osceola City of

Ozark Electric Coop Inc

Petit Jean Electric Coop Corp ..........

Public Service Co of Oklahoma .......

Red River Valley Rit Elec Assn .....

South Central Ark El Coop Inc ........

South Plains Electric Coop Inc ........

Southwestern Electric Power Co .....

Southwestern Public Service Co .....

Verdigris Valley Elec Coop Inc

White River Valley El Coop Inc ........

Woodruff Electric Coop Corp

Western Resources Inc

SPP Total.

(1)

0
0
10
2,792

wscc(U.S.)

Alameda City of

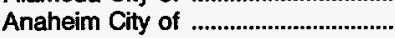

Arizona Electric Pwr Coop Inc ..........

Arizona Public Service Co .................

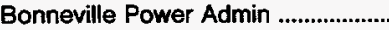

Bountiful City City of .

PUD No 1 of Chelan County

Colton City of

.mpo Power Co .............

Longmont City of

Los Angeles City of

Loveland City of

(n)

Modesto Irrigation District ..................

Montana Power Co ............................

Navopache Electric Coop Inc

Nevada Power Co

Overton Power District No 5 ..............

Pacific Gas \& Electric Co

Palo Alto City of

Pasadena City of ..................................

Public Senvice Co of Colorado

See footnotes at end of table.
114
0
0
8
4
10
6
0
0
0
15
0
0
3
0
6
0
1
5
0
139
0
2
2
0
0
4
9
0
0
10
0
31
26
395

$\begin{aligned} 0 & \\ 0 & \\ 0 & \\ 0 & \\ 0 & \\ 0 & \\ 0 & \\ 0 & \\ 0 & \\ 4 & \\ 1 & \\ 2 & \\ 0 & \\ 0 & \\ 0 & \\ 0 & \\ 0 & \\ 0 & \\ 0 & \\ 72 & \\ 0 & \\ 0 & \\ 0 & \\ 0 & \\ 0 & \\ 0 & \\ 1 & \\ 8 & \\ 24 & \\ 0 & \\ 0 & \\ 0 & \\ 0 & \\ 111 & \end{aligned}$

$\begin{array}{rlll}52 & 0 & 0 & 16 \\ 7 & 0 & 0 & \\ 3 & 0 & 0 & \\ 0 & 0 & 0 & \\ 0 & 0 & 0 & \\ 0 & 0 & 0 & \\ 0 & 0 & 0 & \\ 6 & 0 & 1 & \\ 0 & 0 & 0 & \end{array}$

10

25

1

3

19

1

3

239

2

116

5

4
10

90

24

11

33

30

889

323

36

4
23

1
5
1
580
95
7
20
$*$
44
5
26
8
4
71
2
16
26
8
80
1
837
6
3
122


Fable 19. U.S. Electric Utility Actual Peakload Reductions by North American Electric Reliability Council Region and Hawaii by DSM Program Category, 1993

(Megawatts) (Continued)

\begin{tabular}{|c|c|c|c|c|c|c|}
\hline $\begin{array}{c}\text { North American Electric Rellabillty } \\
\text { Councll Region and Hawall / } \\
\text { Electric Utility }\end{array}$ & $\begin{array}{c}\text { Energy } \\
\text { Efficiency }\end{array}$ & $\begin{array}{l}\text { Direct Load } \\
\text { Control }\end{array}$ & $\begin{array}{l}\text { Interruptible } \\
\text { Load }\end{array}$ & $\begin{array}{l}\text { Other Load } \\
\text { Management }\end{array}$ & $\begin{array}{c}\text { Other Demand- } \\
\text { Side Management }\end{array}$ & $\begin{array}{l}\text { Total DSM } \\
\text { Programs }\end{array}$ \\
\hline \multicolumn{7}{|l|}{ WScc(U.S.) } \\
\hline 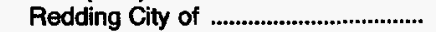 & 22 & 1 & 2 & 2 & 0 & 26 \\
\hline 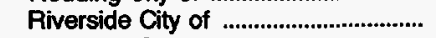 & 2 & 0 & 0 & 4 & : & 6 \\
\hline 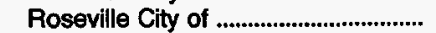 & $*$ & 1 & 0 & 0 & 0 & 2 \\
\hline Sacramento Municipal Util Dist ......... & 62 & 116 & 60 & 22 & 49 & $30 \overline{9}$ \\
\hline Salt River Proj Ag | \& P Dist ............ & 65 & 1 & 0 & 0 & 46 & 112 \\
\hline San Diego Gas \& Electric Co ........... & 25 & 0 & 28 & 3 & $*$ & 57 \\
\hline 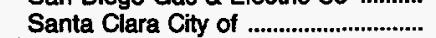 & 0 & 0 & 8 & 0 & 0 & 8 \\
\hline Seattle City of & 38 & 0 & 0 & 0 & 0 & 38 \\
\hline  & 31 & 0 & 0 & 0 & 0 & 31 \\
\hline Southern Calitornia Edison Co ........ & 1,366 & 0 & 0 & 121 & 0 & 1,487 \\
\hline Springfield City of ................................ & 1 & 0 & 0 & 0 & 0 & 1 \\
\hline Sulphur Springs Valley E C Inc ....... & 1 & 2 & 0 & 0 & 0 & 3 \\
\hline Trico Electric Coop Inc ....................... & 0 & 0 & 1 & 0 & 0 & 1 \\
\hline Turlock Irrigation District ....................... & 7 & 0 & 0 & 0 & 0 & 7 \\
\hline Black Hills Corp .................................... & 1 & 2 & 1 & 8 & 0 & 12 \\
\hline Vera Irrigation District \#5 ................. & * & 7 & 0 & 0 & 0 & 7 \\
\hline 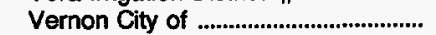 & 0 & 0 & 0 & 8 & 1 & 8 \\
\hline Washington Water Power Co ............ & 103 & 0 & 0 & 0 & 0 & 103 \\
\hline Yellowstone Vily Elec Coop Inc ...... & 0 & 0 & 0 & 4 & 0 & 4 \\
\hline Mohave Electric Coop Inc .................. & 1 & 0 & 0 & 0 & 0 & 1 \\
\hline Tucson Electric Power Co .................. & 14 & 0 & 5 & 0 & 0 & 20 \\
\hline 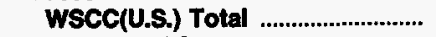 & 2,671 & 183 & 1,003 & 250 & 104 & 4,210 \\
\hline 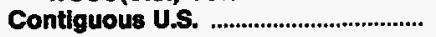 & 10,363 & 3,953 & 6,628 & 1,799 & 315 & 23,057 \\
\hline \multicolumn{7}{|l|}{ Ascc } \\
\hline Alaska Electric Light\&Power Co ...... & 0 & 2 & 0 & 4 & 0 & 7 \\
\hline Golden Valley Elec Assn Inc ............ & * & 0 & 0 & 0 & 0 & * \\
\hline 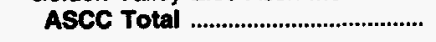 & - & 2 & $\mathbf{0}$ & 4 & 0 & 7 \\
\hline \multicolumn{7}{|l|}{ Hawail } \\
\hline Hawaii Electric Light Co Inc .............. & * & 0 & 0 & 0 & 0 & * \\
\hline Maui Electric Co Ltd .............................. & 2 & 0 & 0 & 0 & 0 & 2 \\
\hline Hawailan Electric Co Inc ..................... & 3 & 0 & 0 & 0 & 0 & 3 \\
\hline 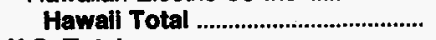 & 5 & 0 & 0 & 0 & 0 & 5 \\
\hline 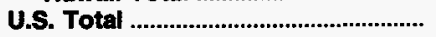 & 10,368 & 3,955 & 6,628 & 1,803 & 315 & 23,069 \\
\hline
\end{tabular}

1 Represents the sum of the achieved reductions in the annual peak load attributable to direct load control, interruptible load, energy efficiency, other load management, and other demand-side management; whereas, in the Electric Power Annual, 1992, 1992 and 1991 peakload reductions attributable to energy efficiency, other load management, and other demand-side management were reported together under conservation and other programs.

- Value less than 0.5 .

Notes: -Data are final. -Data are provided for electric utilities with sales to ultimate consumers or sales for resale greater than or equal to 120,000 megawatthours. -These data reflect actual real changes in the demand for electricity at the time of annual peak load, as opposed to the installed peakload reduction capability (i.e., Potential Peakload Reduction). - Totals may not equal sum of components because of independent rounding.

Source: Energy Information Administration, Form ElA-861, "Annual Electric Utility Report." 
Table 20. U.S. Electric Utility Actual Peakload Reductions by North American Electric Reliability Council Region and Hawail by Class of Ownership and Sector, 1993 (Megawatts)

\begin{tabular}{|c|c|c|c|c|c|c|}
\hline $\begin{array}{l}\text { North American Electric Reliability } \\
\text { Council Region and Hawall / } \\
\text { Electric Utility }\end{array}$ & $\begin{array}{l}\text { Class of } \\
\text { Ownership }\end{array}$ & Residential & Commercial & Industrial & Other & Total \\
\hline 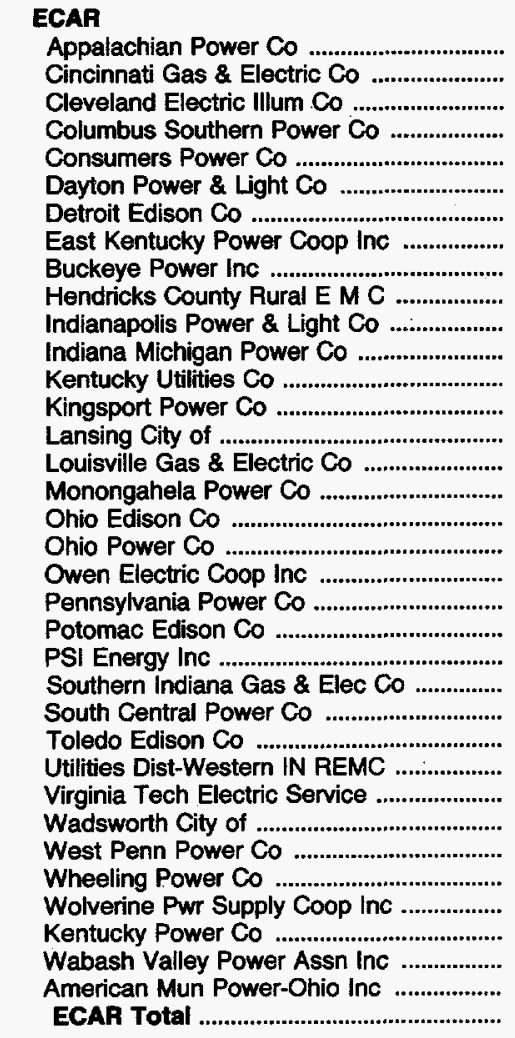 & $\begin{array}{l}\text { Investor-Owned } \\
\text { Investor-Owned } \\
\text { Investor-Owned } \\
\text { Investor-Owned } \\
\text { Investor-Owned } \\
\text { Investor-Owned } \\
\text { Investor-Owned } \\
\text { Cooperative } \\
\text { Cooperative } \\
\text { Cooperative } \\
\text { Investor-Owned } \\
\text { Investor-Owned } \\
\text { Investor-Owned } \\
\text { Investor-Owned } \\
\text { Publicly Owned } \\
\text { Investor-Owned } \\
\text { Investor-Owned } \\
\text { Investor-Owned } \\
\text { Investor-Owned } \\
\text { Cooperative } \\
\text { Investor-Owned } \\
\text { Investor-Owned } \\
\text { Investor-Owned } \\
\text { Investor-Owned } \\
\text { Cooperative } \\
\text { Investor-Owned } \\
\text { Cooperative } \\
\text { Publicly Owned } \\
\text { Publicly Owned } \\
\text { Investor-Owned } \\
\text { Investor-Owned } \\
\text { Cooperative } \\
\text { Investor-Owned } \\
\text { Cooperative } \\
\text { Publicly Owned }\end{array}$ & $\begin{array}{r}14 \\
1 \\
7 \\
38 \\
11 \\
5 \\
11 \\
87 \\
4 \\
* \\
8 \\
11 \\
1 \\
0 \\
0 \\
20 \\
2 \\
21 \\
1 \\
0 \\
71 \\
9 \\
11 \\
19 \\
1 \\
5 \\
* \\
0 \\
14 \\
3 \\
8 \\
2 \\
40 \\
0 \\
423\end{array}$ & $\begin{array}{r}0 \\
6 \\
1 \\
0 \\
40 \\
2 \\
8 \\
0 \\
0 \\
0 \\
4 \\
0 \\
* \\
0 \\
* \\
0 \\
24 \\
4 \\
0 \\
* \\
0 \\
30 \\
29 \\
1 \\
3 \\
2 \\
* \\
* \\
0 \\
24 \\
0 \\
0 \\
0 \\
0 \\
0 \\
179\end{array}$ & $\begin{array}{r}132 \\
17 \\
3 \\
20 \\
57 \\
3 \\
13 \\
0 \\
32 \\
* \\
9 \\
29 \\
0 \\
0 \\
0 \\
64 \\
33 \\
247 \\
99 \\
* \\
40 \\
58 \\
37 \\
1 \\
4 \\
2 \\
5 \\
* \\
8 \\
108 \\
0 \\
0 \\
33 \\
0 \\
5 \\
058\end{array}$ & $\begin{array}{l}0 \\
0 \\
0 \\
0 \\
0 \\
0 \\
0 \\
0 \\
0 \\
0 \\
0 \\
0 \\
9 \\
0 \\
0 \\
0 \\
0 \\
0 \\
0 \\
0 \\
0 \\
0 \\
0 \\
0 \\
0 \\
0 \\
0 \\
1 \\
0 \\
0 \\
0 \\
0 \\
0 \\
0 \\
1 \\
11\end{array}$ & $\begin{array}{r}146 \\
22 \\
6 \\
27 \\
135 \\
16 \\
25 \\
11 \\
119 \\
4 \\
14 \\
37 \\
20 \\
1 \\
* \\
64 \\
76 \\
252 \\
120 \\
1 \\
40 \\
158 \\
76 \\
13 \\
25 \\
5 \\
11 \\
2 \\
8 \\
145 \\
3 \\
8 \\
35 \\
40 \\
6 \\
1,671\end{array}$ \\
\hline 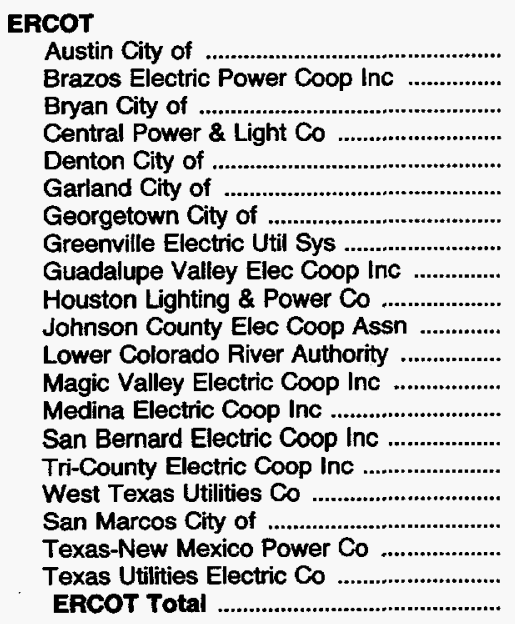 & $\begin{array}{l}\text { Publicly Owned } \\
\text { Cooperative } \\
\text { Publicly Owned } \\
\text { Investor-Owned } \\
\text { Publicly Owned } \\
\text { Publicly Owned } \\
\text { Publicly Owned } \\
\text { Publicly Owned } \\
\text { Cooperative } \\
\text { Investor-Owned } \\
\text { Cooperative } \\
\text { Publicly Owned } \\
\text { Cooperative } \\
\text { Cooperative } \\
\text { Cooperative } \\
\text { Cooperative } \\
\text { Investor-Owned } \\
\text { Publicly Owned } \\
\text { Investor-Owned } \\
\text { Investor-Owned }\end{array}$ & $\begin{array}{r}152 \\
1 \\
6 \\
30 \\
2 \\
6 \\
* \\
0 \\
5 \\
28 \\
* \\
69 \\
* \\
0 \\
10 \\
2 \\
0 \\
3 \\
11 \\
478 \\
802\end{array}$ & $\begin{array}{r}60 \\
0 \\
4 \\
41 \\
1 \\
\vdots \\
0 \\
* \\
18 \\
2 \\
3 \\
0 \\
0 \\
0 \\
0 \\
0 \\
1 \\
1 \\
336 \\
467\end{array}$ & $\begin{array}{r}0 \\
0 \\
0 \\
0 \\
0 \\
8 \\
0 \\
3 \\
50 \\
2 \\
0 \\
20 \\
0 \\
0 \\
4 \\
0 \\
39 \\
0 \\
8 \\
0 \\
133\end{array}$ & 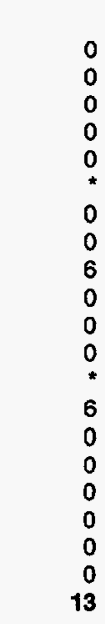 & $\begin{array}{r}212 \\
1 \\
9 \\
71 \\
3 \\
14 \\
3 \\
31 \\
48 \\
2 \\
92 \\
7 \\
6 \\
14 \\
2 \\
39 \\
3 \\
19 \\
814 \\
1,414\end{array}$ \\
\hline 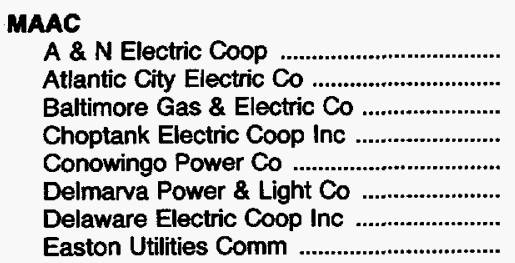 & $\begin{array}{l}\text { Cooperative } \\
\text { Investor-Owned } \\
\text { Investor-Owned } \\
\text { Cooperative } \\
\text { Investor-Owned } \\
\text { Investor-Owned } \\
\text { Cooperative } \\
\text { Publicly Owned }\end{array}$ & $\begin{array}{r}1 \\
56 \\
15 \\
4 \\
3 \\
51 \\
6 \\
\end{array}$ & $\begin{array}{r}0 \\
25 \\
44 \\
0 \\
0 \\
66 \\
0 \\
0\end{array}$ & $\begin{array}{r}0 \\
13 \\
1 \\
0 \\
45 \\
0 \\
0\end{array}$ & $\begin{array}{l}0 \\
0 \\
0 \\
0 \\
0 \\
0 \\
0 \\
0\end{array}$ & $\begin{array}{r}1 \\
94 \\
59 \\
5 \\
3 \\
162 \\
6 \\
*\end{array}$ \\
\hline
\end{tabular}

See footnotes at end of table. 
Table 20. U.S. Electric Utility Actual Peakload Reductions by North American Electric Reliability Council Region and Hawail by Class of Ownership and Sector, 1993 (Megawatts) (Continued)

\begin{tabular}{|c|c|c|c|c|c|c|}
\hline $\begin{array}{l}\text { North American Electric Reliability } \\
\text { Council Region and Hawail / } \\
\text { Electric Utllity }\end{array}$ & $\begin{array}{l}\text { Class of } \\
\text { Ownership }\end{array}$ & Residential & Commerclal & Industrial & Other & Total \\
\hline
\end{tabular}

MAAC (Continued)

Jersey Central Power\&Light Co

Metropolitan Edison Co

Pennsylvania Electric Co

Pennsylvania Power \& Light Co

Philadelphia Electric Co .

Potomac Electric Power Co

Public Senvice Electric\&Gas $\mathrm{Co}$

Southern Maryland El Coop Inc

Somerset Rural Elec Coop Inc

Adams Electric Coop Inc

Bedford Rural Elec Coop Inc

Valley Rural Electric Coop Inc

Central Electric Coop Inc

Claverack Rural Elec Coop Inc

Northwestern Rural E C A inc

MAAC Total

MAIN

Boone Electric Coop

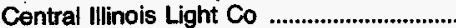

Coles-Moultrie Electric Coop

Columbia City of

Commonwealth Edison Co

Cuivre River Electric Coop Inc

Eastern Illini Electric Coop

Illinois Power Co

Madison Gas \& Electric $\mathrm{Co}$

Manitowoc Public Utilities

Marshfield City of .

Shelby Electric Coop Inc .........

Southwestern Electric Coop Inc

Springfield City of

Tri-County Electric Coop inc

Wisconsin Electric Power Co

Wisconsin Power \& Light Co

Wisconsin Public Power Inc Sys

Wisconsin Public Service Corp

MAIN Total.

MAPP(U.S.)

Ames Gity of

Anoka City of

Austin City of

Barron Electric Coop

Beatrice City of

Cedar Falls City of

Central lowa Power Coop ..

Central Power Elec Coop Inc

Clark Electric Coop

Coop Power Assn

Cornhusker Public Power Dist

Dawson County Public Pwr Dist

East River Elec Power Coop Inc

Fairmont Public Utilities Comm

Freeborn-Mower Electric Coop

Grant-Lafayette Electric Coop

IES Utilities Inc

Interstate Power Co

lowa Lakes Electric CoOp

L \& O Power Coop

Lincoln Electric System

Marshall Gity of

Nodak Electric Coop inc

Midland Power Coop

Minnesota Power \& Light Co
Investor-Owned Investor-Owned Investor-Owned Investor-Owned Investor-Owned Investor-Owned Investor-Owned Cooperative Cooperative

Cooperative

Cooperative

Cooperative

Cooperative

Cooperative

Cooperative

Cooperative

Investor-Owned

Cooperative

Publicly Owned

Investor-Owned

Cooperative

Cooperative

Cooperative

Investor-Owned

Investor-Owned

Publicly Owned

Publicly Owned

Cooperative

Cooperative

Publicly Owned

Cooperative

Investor-Owned

Investor-Owned

Investor-Owned

Publicly Owned

Investor-Owned

Publicly Owned Publicly Owned Publicly Owned Cooperative

Publicly Owned

Publicly Owned

Cooperative

Cooperative

Cooperative

Cooperative

Publicly Owned

Publicly Owned

Cooperative

Publicly Owned

Cooperative

Cooperative

Investor-Owned Investor-Owned

Cooperative

Cooperative

Publicly Owned

Publicly Owned

Investor-Owned

Cooperative

Cooperative

Investor-Owned

$\begin{array}{rrrrr}50 & 27 & 68 & 0 & 144 \\ 90 & 39 & 147 & 0 & 276 \\ 12 & 10 & 29 & 0 & 51 \\ 6 & 2 & 159 & 0 & 167 \\ 44 & 1 & 0 & 0 & 45 \\ 39 & 138 & 0 & 0 & 177 \\ 66 & 100 & 78 & 0 & 244 \\ 32 & 0 & 0 & 0 & 32 \\ 1 & 0 & 0 & 0 & 1 \\ 8 & 0 & 0 & 0 & 8 \\ 2 & 0 & 0 & * & 2 \\ 3 & 0 & 0 & 0 & 3 \\ 4 & 0 & 0 & 0 & 4 \\ 5 & 0 & 0 & 0 & 5 \\ 5 & 0 & 0 & 0 & 5 \\ 502 & 451 & 540 & 0 & 1,493\end{array}$

$\begin{array}{rrrrr}2 & 0 & 0 & 0 & 2 \\ 3 & 62 & 0 & 0 & 62 \\ 5 & 0 & 5 & 0 & 8 \\ 2 & 1 & 1 & 0 & 7 \\ 4 & 20 & 1 & 0 & 23 \\ 3 & 3 & 0 & 0 & 6 \\ 6 & 3 & 0 & 0 & 6 \\ 0 & 0 & 4 & 0 & 10 \\ 4 & 5 & 0 & 0 & 5 \\ 1 & 25 & 1 & 1 & 30 \\ * & 1 & 6 & 0 & 2 \\ 0 & 2 & 0 & 0 & 1 \\ 20 & 2 & 3 & 0 & 8 \\ 4 & 5 & 145 & 0 & 25 \\ 2 & 1 & 53 & 0 & 5 \\ 0 & 7 & 4 & 0 & 12 \\ 136 & 0 & 3 & 0 & 145 \\ 5 & 147 & 0 & 0 & 336 \\ 1 & 45 & 226 & 0 & 54 \\ 33 & 2 & 32 & 1 & 7 \\ 230 & 380 & & 6 & 91 \\ & 50 & 0 & 8 & 844\end{array}$

See footnotes at end of table. 
Table 20. U.S. Electric Utility Actual Peakload Reductions by North American Electric Reliability Council Region and Hawaii by Class of Ownership and Sector, 1993 (Megawatts) (Continued)

\begin{tabular}{c|c|c|c|c|c|c|c|c|}
\hline $\begin{array}{c}\text { North American Electric Reliability } \\
\text { Council Region and Hawail / } \\
\text { Electric Utility }\end{array}$ & $\begin{array}{c}\text { Class of } \\
\text { Ownership }\end{array}$ & Residential & Commercial & Industrial \\
\hline
\end{tabular}

MAPP(U.S.) (Continued)

Minnkota Power Coop Inc

Moorhead City of

Norris Public Power District

North Platte City of

Northem States Power Co

Northern States Power Co

Northwest lowa Power Coop

Northwestern Public Senvice Co

Oakdale Electric Coop

Oliver-Mercer Elec Coop Inc

Omaha Public Power District

Otter Tail Power Co

Owatonna City of

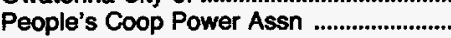

Pella City of

Pierre City of

Polk-Burnett Electric Coop

Rochester Public Utilities

Roseau Electric Coop Inc

Runestone Electric Assn

Shakopee Public Utilities Comm

Superior Water Light\&Power Co

Tri-County Electric Coop

Verendrye Electric Coop Inc

Vernon Electric Coop

Mountrail-Williams El Coop Inc

Wild Rice Electric Coop Inc

Municipal Energy Agency of NE

Midwest Power Systems inc

Cass County Electric Coop Inc

MAPP(U.S.) Total

NPCC(U.S.)

Bangor Hydro-Electric $C_{0}$

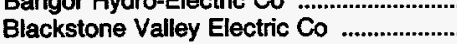

Boston Edison Co

Braintree Town of

Burlington City of

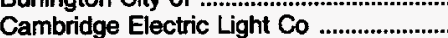

Central Hudson Gas \& Elec Corp

Central Maine Power Co

Central Vermont Pub Serv Corp

Chicopee City of

Commonwealth Electric $\mathrm{Co}$

Concord Electric Co

Connecticut Light \& Power Co

Connecticut Valley Elec Co Inc

Consolidated Edison Co-NY Inc

Eastern Edison Co

Exeter \& Hampton Electric Co

Fitchburg Gas \& Elec Light Co

Green Mountain Power Corp

Holyoke City of

Hingham City of

Jamestown City of

Long Island Lighting Co

Maine Public Service Co

Massachusetts Electric Co

Massena Town of

Montaup Electric Co

Narragansett Electric $\mathrm{Co}$

New Hampshire Elec Coop Inc

New York State Elec \& Gas Corp .............

Newport Electric Corp

Niagara Mohawk Power Corp
Cooperative

Publicly Owned

Publicly Owned

Publicly Owned

Publicly Owned

Investor-Owned

Investor-Owned

Cooperative

Investor-Owned

Cooperative

Cooperative

Publicly Owned

Investor-Owned

Publicly Owned

Cooperative

Publicly Owned

Publicly Owned

Cooperative

Publicly Owned

Cooperative

Cooperative

Publicly Owned

Investor-Owned

Cooperative

Cooperative

Cooperative

Cooperative

Cooperative

Cooperative

Publicly Owned

Investor-Owned

Cooperative

Investor-Owned Investor-Owned Investor-Owned

Publicly Owned

Publicly Owned

Investor-Owned

Investor-Owned

Investor-Owned

Investor-Owned

Publicly Owned

Investor-Owned

Investor-Owned

Investor-Owned

Investor-Owned

Investor-Owned

Investor-Owned

Investor-Owned

Investor-Owned

Investor-Owned

Investor-Owned

Publicly Owned

Publicly Owned

Publicly Owned

Investor-Owned

Investor-Owned

Investor-Owned

Publicly Owned

Investor-Owned

Investor-Owned

Cooperative

Investor-Owned

Investor-Owned

Investor-Owned

265

9

0
3

41

140

140
.6

20
0
1
1
85
278
0
0
0
0
0
25
4
0
0
1
0
2
0
2
1
0
0
0
1
0
0
1
9
17
8
508

0
3


Table 20. U.S. Electric Utility Actual Peakload Reductions by North American Electric Reliability Council Region and Hawaii by Class of Ownership and Sector, 1993 (Megawatts) (Continued)

\begin{tabular}{|c|c|c|c|c|c|c|}
\hline $\begin{array}{l}\text { North American Electric Rellability } \\
\text { Councll Region and Hawail / } \\
\text { Electric Utility }\end{array}$ & $\begin{array}{c}\text { Class of } \\
\text { Ownership }\end{array}$ & Residential & Commercial & Industrial & Other & Total \\
\hline  & $\begin{array}{l}\text { Publicly Owned } \\
\text { Investor-Owned } \\
\text { Publicly Owned } \\
\text { Investor-Owned } \\
\text { Publicly Owned } \\
\text { Investor-Owned } \\
\text { Publicly Owned } \\
\text { Publicly Owned } \\
\text { Investor-Owned } \\
\text { Investor-Owned } \\
\text { Investor-Owned } \\
\text { Investor-Owned }\end{array}$ & $\begin{array}{r}* \\
21 \\
13 \\
* \\
* \\
10 \\
1 \\
* \\
13 \\
* \\
34 \\
2 \\
574\end{array}$ & $\begin{array}{r}78 \\
20 \\
* \\
6 \\
16 \\
\vdots \\
4 \\
18 \\
0 \\
21 \\
6 \\
1,184\end{array}$ & $\begin{array}{r} \\
0 \\
0 \\
0 \\
0 \\
24 \\
1 \\
0 \\
26 \\
0 \\
2 \\
1 \\
191\end{array}$ & $\begin{array}{c}0 \\
0 \\
0 \\
0 \\
0 \\
0 \\
\vdots \\
0 \\
0 \\
0 \\
1 \\
0 \\
19\end{array}$ & $\begin{array}{r}1 \\
98 \\
33 \\
* \\
6 \\
49 \\
3 \\
4 \\
57 \\
\vdots \\
59 \\
8 \\
1,968\end{array}$ \\
\hline 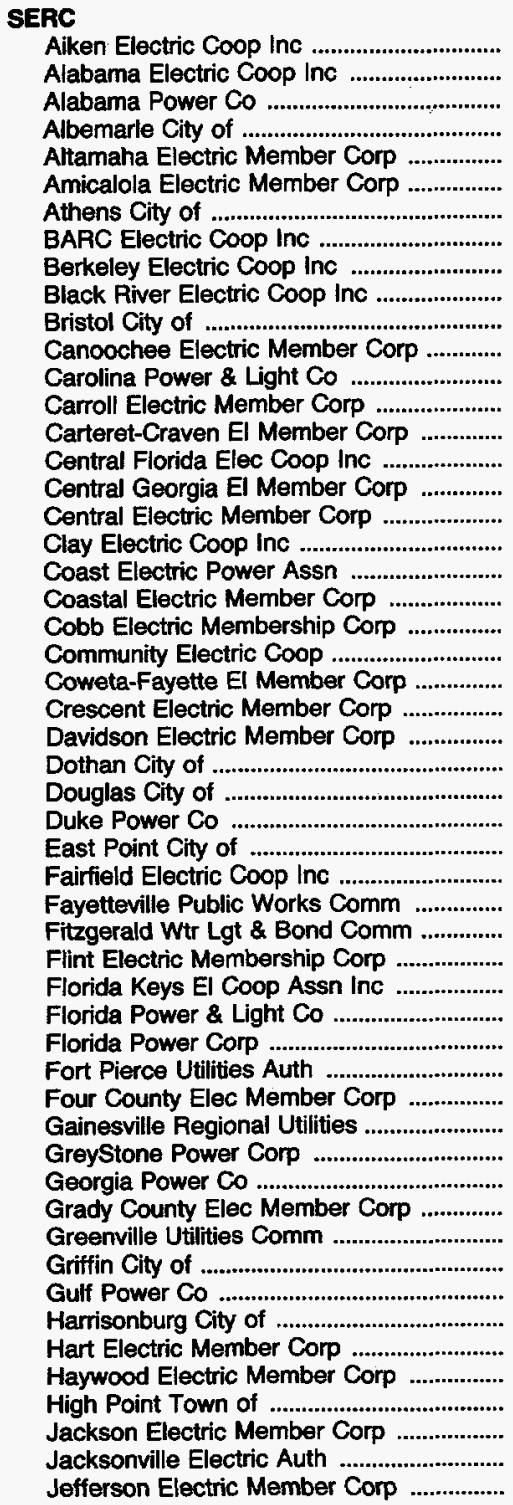 & $\begin{array}{l}\text { Cooperative } \\
\text { Cooperative } \\
\text { Investor-Owned } \\
\text { Publicly Owned } \\
\text { Cooperative } \\
\text { Cooperative } \\
\text { Publicly Owned } \\
\text { Cooperative } \\
\text { Cooperative } \\
\text { Cooperative } \\
\text { Publicly Owned } \\
\text { Cooperative } \\
\text { Investor-Owned } \\
\text { Cooperative } \\
\text { Cooperative } \\
\text { Cooperative } \\
\text { Cooperative } \\
\text { Cooperative } \\
\text { Cooperative } \\
\text { Cooperative } \\
\text { Cooperative } \\
\text { Cooperative } \\
\text { Cooperative } \\
\text { Cooperative } \\
\text { Cooperative } \\
\text { Cooperative } \\
\text { Publicly Owned } \\
\text { Publicly Owned } \\
\text { Investor-Owned } \\
\text { Publicly Owned } \\
\text { Cooperative } \\
\text { Publicly Owned } \\
\text { Publicly Owned } \\
\text { Cooperative } \\
\text { Cooperative } \\
\text { Investor-Owned } \\
\text { Investor-Owned } \\
\text { Publicly Owned } \\
\text { Cooperative } \\
\text { Publicly Owned } \\
\text { Cooperative } \\
\text { Investor-Owned } \\
\text { Cooperative } \\
\text { Publicly Owned } \\
\text { Publicly Owned } \\
\text { Investor-Owned } \\
\text { Publicly Owned } \\
\text { Cooperative } \\
\text { Cooperative } \\
\text { Publicly Owned } \\
\text { Cooperative } \\
\text { Publicly Owned } \\
\text { Cooperative }\end{array}$ & 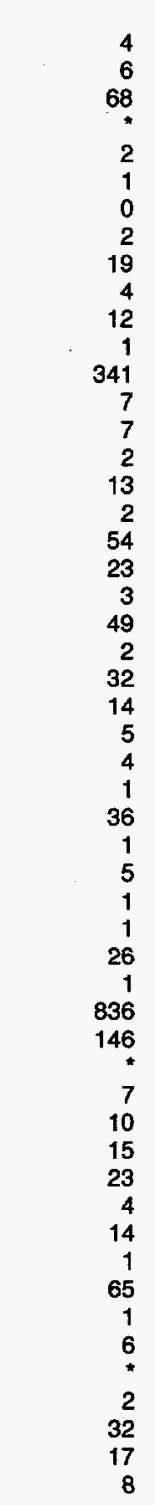 & 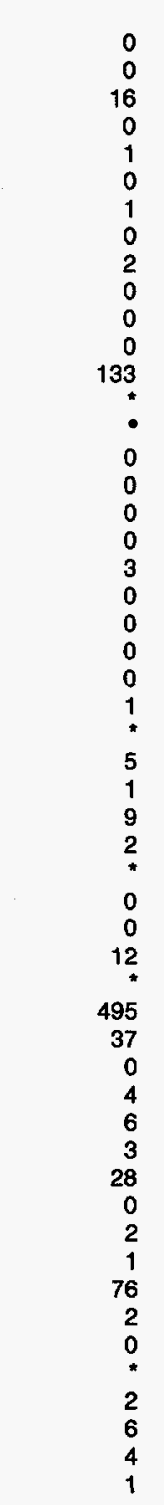 & 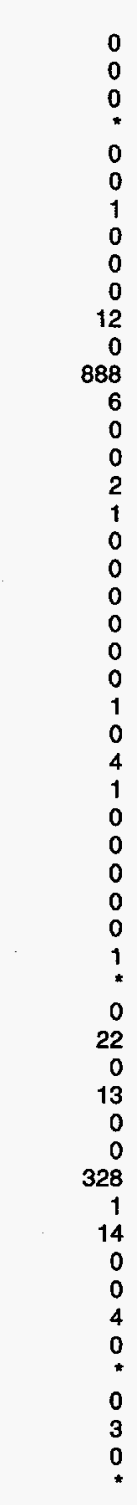 & $\begin{array}{r}0 \\
0 \\
0 \\
0 \\
0 \\
0 \\
0 \\
0 \\
0 \\
0 \\
3 \\
1 \\
0 \\
0 \\
0 \\
0 \\
0 \\
0 \\
0 \\
0 \\
0 \\
0 \\
0 \\
0 \\
0 \\
0 \\
0 \\
0 \\
0 \\
0 \\
0 \\
0 \\
0 \\
0 \\
0 \\
0 \\
0 \\
0 \\
0 \\
0 \\
0 \\
0 \\
0 \\
0 \\
0 \\
0 \\
0 \\
0 \\
0 \\
0 \\
0\end{array}$ & 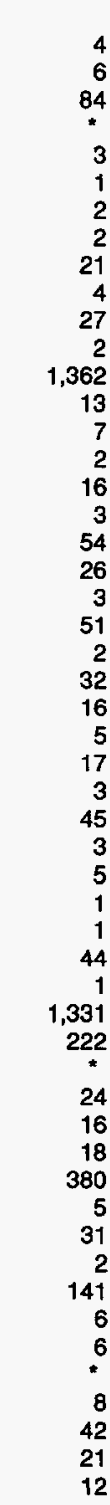 \\
\hline
\end{tabular}

See footnotes at end of table. 
Table 20. U.S. Electric Utility Actual Peakload Reductions by North American Electric Reliability Council Region and Hawaii by Class of Ownership and Sector, 1993 (Megawatts) (Continued)

\begin{tabular}{c|c|c|c|c|c|c|}
\hline $\begin{array}{c}\text { North American Electric Reliability } \\
\text { Council Region and Hawall } / \\
\text { Electric Utility }\end{array}$ & $\begin{array}{c}\text { Class of } \\
\text { Ownership }\end{array}$ & Residential & Commercial & Industrial & Other \\
\hline
\end{tabular}

SERC (Continued)

Jones-Onslow Elec Member Corp ............ Kinston City of

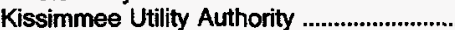

Lakeland City of

Lamar Electric Membership Corp

Laurens Electric Coop Inc

Laurinburg City of

Lawrenceville City of

Lee County Electric Coop Inc

Leesburg City of

Lumbee River Elec Member Corp ............

Lumberton City of

Lynches River Elec Coop Inc

Manassas City of

Marietta City of

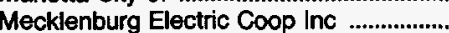

Mid-Carolina Electric Coop Inc

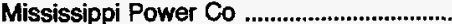

Monroe City of

New Bern City of

New River Light \& Power Co

North Carolina Mun Power Agny

Northern Virginia Elec Coop ..

North Carolina Eastern M P A

Northern Neck Elec Coop Ino

Ocala City of

Orangeburg City of

Palmetto Electric Coop inc

Orlando Utilities Comm

Pee Dee Electric Member Corp

Piedmont Electric Member Corp

ce George Electric COOp

Pulaski City of

Randolph Electric Member Corp

Rayle Electric Membership Corp

Reedy Creek Improvement Dist

Rock Hill City of

Rocky Mount City of

Rutherford Elec Member Corp

Satilla Rural Elec Member Corp

Sawnee Electric Members Corp

Shenandoah Valley Elec Coop.

South Carolina Pub Serv Auth

South Mississippi El Pwr Assn ...

Snapping Shoals El Member Corp ...........

Sumter Electric Coop Inc

Tallahassee City of

Tampa Electric Co

Tennessee Valley Authority

Thomasville City of

Tri-County Elec Member Corp ...........

Tideland Electric Member Corp

Union Electric Membership Corp ...............

Virginia Electric \& Power Co ...

Wake Electric Membership Corp .............

Walton Electric Member Corp

Washington City of

Washington Elec Member Corp

Withlacoochee River Elec Coop

York Electric Coop Inc

Southside Electric Coop Inc

Brunswick Electric Member Corp

Colquitt Electric Members Corp

Central Electric Pwr Coop Inc

Rappahannock Electric Coop

SERC Total
Cooperative

Publicly Owned

Publicly Owned

Publicly Owned

Cooperative

Cooperative

Publicly Owned

Publicly Owned

Cooperative

Publicly Owned

Cooperative

Publicly Owned

Cooperative

Publicly Owned

Publicly Owned

Cooperative

Cooperative

Investor-Owned

Publicly Owned

Publicly Owned

Publicly Owned

Publicly Owned

Cooperative

Publicly Owned

Cooperative

Publicly Owned

Publicly Owned

Cooperative

Publicly Owned

Cooperative

Cooperative

Cooperative

Publicly Owned

Cooperative

Cooperative

Publicly Owned

Publicly Owned

Publicly Owned

Cooperative

Cooperative

Cooperative

Cooperative

Cooperative

Investor-Owned

Publicly Owned

Cooperative

Cooperative

Cooperative

Publicly Owned

Investor-Owned

Federal

Publicly Owned

Cooperative

Cooperative

Cooperative

Investor-Owned

Cooperative

Cooperative

Publicly Owned

Cooperative

Cooperative

Cooperative

Cooperative

Cooperative

Cooperative

Cooperative

Cooperative
10

0

1

0

*

2

44

2

2

6

6

5

1

19
18

33

2

4
2

5

17

11

1

15

1

0

5
14

14

14

7

2
62

25

3

7
19
57

57
362

586
4

2

6
8
16

16
10

10
21

4

29

1
6

14

53

20
3,605



*

0

0

0

2

0

0

0
16

0
0

0

0

Ses footnotes at end of table. 
Table 20. U.S. Electric Utility Actual Peakload Reductions by North American Electric Reliability Council Region and Hawail by Class of Ownership and Sector, 1993 (Megawatts) (Continued)

\begin{tabular}{|c|c|c|c|c|c|c|}
\hline $\begin{array}{l}\text { North American Electric Reliablilty } \\
\text { Council Region and Hawail / } \\
\text { Electric Utilty }\end{array}$ & $\begin{array}{l}\text { Class of } \\
\text { Ownership }\end{array}$ & Residential & Commercial & Industrial & Other & Total \\
\hline 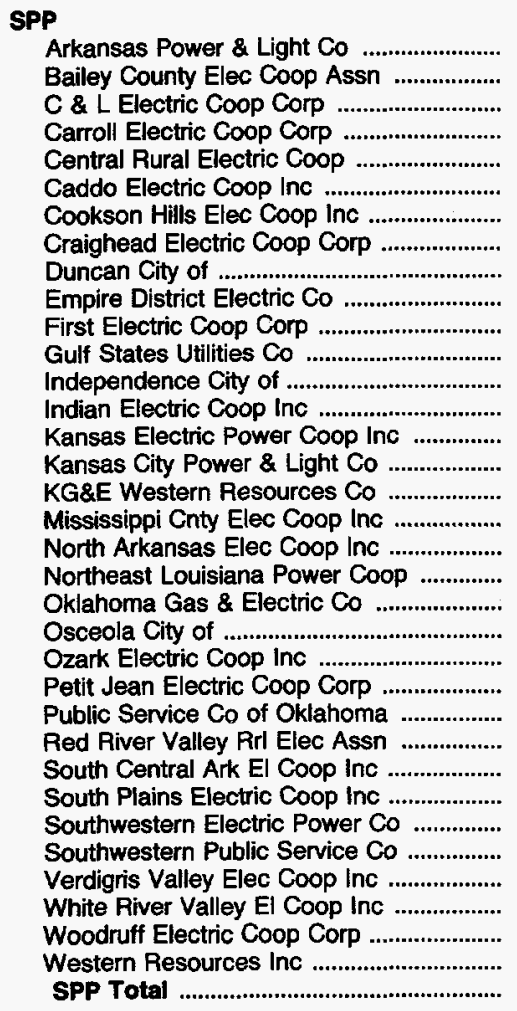 & $\begin{array}{l}\text { Investor-Owned } \\
\text { Cooperative } \\
\text { Cooperative } \\
\text { Cooperative } \\
\text { Cooperative } \\
\text { Cooperative } \\
\text { Cooperative } \\
\text { Cooperative } \\
\text { Publicly Owned } \\
\text { Investor-Owned } \\
\text { Cooperative } \\
\text { Investor-Owned } \\
\text { Publicly Owned } \\
\text { Cooperative } \\
\text { Cooperative } \\
\text { Investor-Owned } \\
\text { Investor-Owned } \\
\text { Cooperative } \\
\text { Cooperative } \\
\text { Cooperative } \\
\text { Investor-Owned } \\
\text { Publicly Owned } \\
\text { Cooperative } \\
\text { Cooperative } \\
\text { Investor-Owned } \\
\text { Cooperative } \\
\text { Cooperative } \\
\text { Cooperative } \\
\text { Investor-Owned } \\
\text { Investor-Owned } \\
\text { Cooperative } \\
\text { Cooperative } \\
\text { Cooperative } \\
\text { Investor-Owned }\end{array}$ & $\begin{array}{r}77 \\
0 \\
0 \\
8 \\
3 \\
0 \\
6 \\
1 \\
* \\
0 \\
12 \\
1 \\
2 \\
2 \\
0 \\
6 \\
0 \\
0 \\
5 \\
0 \\
191 \\
0 \\
2 \\
2 \\
0 \\
* \\
0 \\
1 \\
8 \\
22 \\
10 \\
0 \\
1 \\
26 \\
385\end{array}$ & $\begin{array}{r}23 \\
0 \\
0 \\
* \\
0 \\
\\
0 \\
\\
0 \\
2 \\
* \\
0 \\
1 \\
0 \\
13 \\
0 \\
1 \\
0 \\
3 \\
27 \\
0 \\
0 \\
* \\
0 \\
1 \\
0 \\
0 \\
0 \\
* \\
0 \\
7 \\
0 \\
0 \\
78\end{array}$ & $\begin{array}{r}66 \\
7 \\
3 \\
0 \\
1 \\
10 \\
0 \\
6 \\
0 \\
25 \\
7 \\
* \\
0 \\
1 \\
8 \\
0 \\
15 \\
0 \\
0 \\
0 \\
21 \\
3 \\
0 \\
0 \\
116 \\
4 \\
4 \\
0 \\
82 \\
1 \\
1 \\
0 \\
2 \\
4 \\
386\end{array}$ & $\begin{array}{r}0 \\
0 \\
0 \\
0 \\
0 \\
0 \\
0 \\
0 \\
0 \\
0 \\
0 \\
0 \\
0 \\
0 \\
0 \\
0 \\
0 \\
0 \\
0 \\
0 \\
0 \\
0 \\
0 \\
0 \\
0 \\
0 \\
0 \\
9 \\
0 \\
0 \\
0 \\
0 \\
30 \\
0 \\
39\end{array}$ & $\begin{array}{r}166 \\
7 \\
3 \\
8 \\
4 \\
10 \\
6 \\
6 \\
4 \\
25 \\
20 \\
1 \\
2 \\
3 \\
8 \\
19 \\
15 \\
1 \\
5 \\
3 \\
239 \\
3 \\
2 \\
3 \\
116 \\
5 \\
4 \\
10 \\
90 \\
24 \\
11 \\
7 \\
33 \\
30 \\
889\end{array}$ \\
\hline 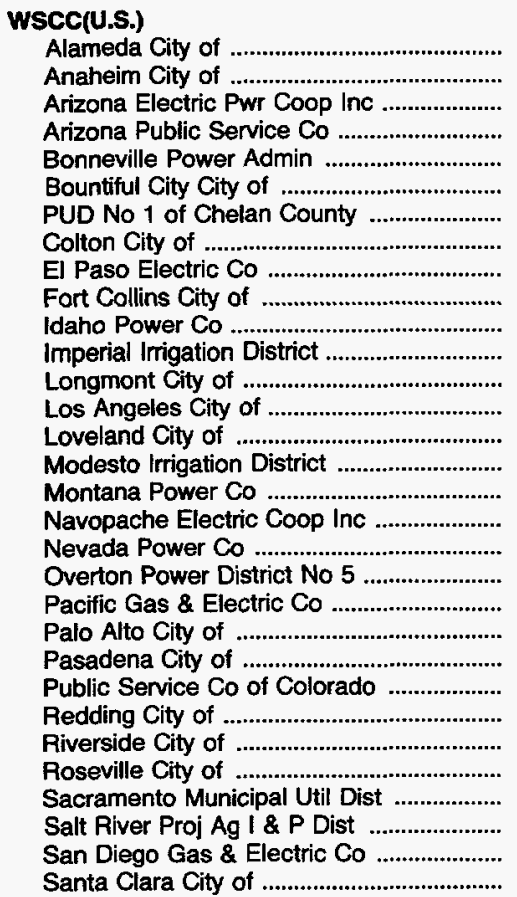 & $\begin{array}{l}\text { Publicly Owned } \\
\text { Publicly Owned } \\
\text { Cooperative } \\
\text { Investor-Owned } \\
\text { Federal } \\
\text { Publicly Owned } \\
\text { Publicly Owned } \\
\text { Publicly Owned } \\
\text { Investor-Owned } \\
\text { Publicly Owned } \\
\text { Investor-Owned } \\
\text { Publicly Owned } \\
\text { Publicly Owned } \\
\text { Publicly Owned } \\
\text { Publicly Owned } \\
\text { Publicly Owned } \\
\text { Investor-Owned } \\
\text { Cooperative } \\
\text { Investor-Owned } \\
\text { Publicly Owned } \\
\text { Investor-Owned } \\
\text { Publicly Owned } \\
\text { Publicly Owned } \\
\text { Investor-Owned } \\
\text { Publicly Owned } \\
\text { Publicly Owned } \\
\text { Publicly Owned } \\
\text { Publicly Owned } \\
\text { Publicly Owned } \\
\text { Investor-Owned } \\
\text { Publicly Owned }\end{array}$ & $\begin{array}{r}* \\
2 \\
* \\
330 \\
0 \\
* \\
0 \\
* \\
0 \\
2 \\
16 \\
7 \\
1 \\
26 \\
1 \\
13 \\
5 \\
5 \\
51 \\
* \\
84 \\
0 \\
2 \\
6 \\
20 \\
5 \\
2 \\
168 \\
85 \\
7 \\
0\end{array}$ & $\begin{array}{r} \\
1 \\
106 \\
0 \\
0 \\
0 \\
0 \\
10 \\
0 \\
2 \\
1 \\
3 \\
37 \\
0 \\
2 \\
13 \\
1 \\
28 \\
1 \\
172 \\
6 \\
2 \\
29 \\
4 \\
* \\
140 \\
27 \\
50 \\
0\end{array}$ & $\begin{array}{r}0 \\
2 \\
0 \\
144 \\
95 \\
7 \\
20 \\
0 \\
34 \\
3 \\
2 \\
0 \\
* \\
8 \\
0 \\
0 \\
0 \\
1 \\
1 \\
0 \\
535 \\
0 \\
0 \\
87 \\
2 \\
* \\
0 \\
0 \\
* \\
0 \\
8\end{array}$ & $\begin{array}{r}1 \\
0 \\
0 \\
0 \\
0 \\
0 \\
0 \\
0 \\
0 \\
0 \\
5 \\
\\
\\
0 \\
1 \\
0 \\
9 \\
1 \\
0 \\
* \\
45 \\
0 \\
0 \\
0 \\
1 \\
0 \\
0 \\
1 \\
0 \\
0 \\
0\end{array}$ & $\begin{array}{r}1 \\
5 \\
1 \\
580 \\
95 \\
7 \\
20 \\
\star \\
44 \\
5 \\
26 \\
8 \\
4 \\
71 \\
2 \\
16 \\
26 \\
8 \\
80 \\
1 \\
837 \\
6 \\
3 \\
122 \\
26 \\
6 \\
2 \\
309 \\
112 \\
57 \\
8\end{array}$ \\
\hline
\end{tabular}

See footnotes at end of table. 
Table 20. U.S. Electric Utility Actual Peakload Reductions by North American Electric Reliability Council Region and Hawaii by Class of Ownership and Sector, 1993 (Megawatts) (Continued)

\begin{tabular}{|c|c|c|c|c|c|c|}
\hline $\begin{array}{c}\text { North American Electric Reliability } \\
\text { Council Region and Hawall / } \\
\text { Electric Utility }\end{array}$ & $\begin{array}{l}\text { Class of } \\
\text { Ownership }\end{array}$ & Residential & Commerclal & Industrial & Other & Total \\
\hline 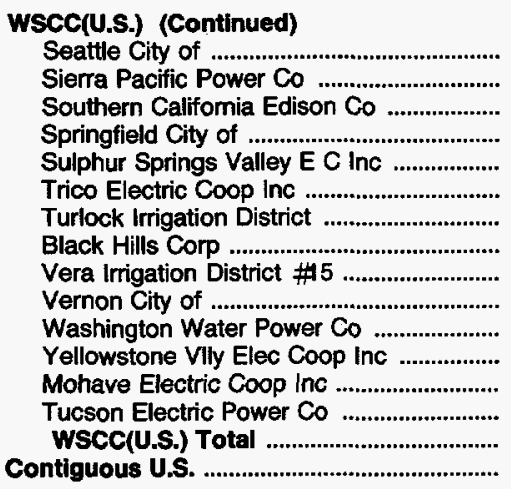 & $\begin{array}{l}\text { Publicly Owned } \\
\text { Investor-Owned } \\
\text { Investor-Owned } \\
\text { Publicly Owned } \\
\text { Cooperative } \\
\text { Cooperative } \\
\text { Publicly Owned } \\
\text { Investor-Owned } \\
\text { Publicly Owned } \\
\text { Publicly Owned } \\
\text { Investor-Owned } \\
\text { Cooperative } \\
\text { Cooperative } \\
\text { Investor-Owned }\end{array}$ & $\begin{array}{r}17 \\
4 \\
360 \\
1 \\
\star \\
0 \\
5 \\
4 \\
7 \\
0 \\
97 \\
4 \\
\text { * } \\
5 \\
1,342 \\
8,846\end{array}$ & $\begin{array}{r}16 \\
11 \\
749 \\
\star \\
* \\
1 \\
1 \\
* \\
0 \\
0 \\
4 \\
0 \\
* \\
10 \\
1,428 \\
7,534\end{array}$ & $\begin{array}{r}2 \\
17 \\
335 \\
* \\
0 \\
0 \\
1 \\
8 \\
0 \\
8 \\
2 \\
0 \\
0 \\
5 \\
1,329 \\
6,270\end{array}$ & $\begin{array}{r}3 \\
0 \\
43 \\
0 \\
2 \\
0 \\
\star \\
* \\
0 \\
0 \\
0 \\
0 \\
0 \\
0 \\
112 \\
407\end{array}$ & $\begin{array}{r}38 \\
31 \\
1,487 \\
1 \\
3 \\
1 \\
7 \\
12 \\
7 \\
6 \\
103 \\
4 \\
1 \\
20 \\
4,210 \\
23,057\end{array}$ \\
\hline 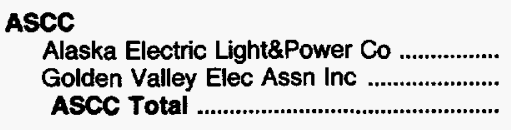 & $\begin{array}{l}\text { Investor-Owned } \\
\text { Cooperative }\end{array}$ & $\begin{array}{l}4 \\
* \\
4\end{array}$ & $\begin{array}{l}3 \\
0 \\
3\end{array}$ & $\begin{array}{l}0 \\
0 \\
0\end{array}$ & $\begin{array}{l}0 \\
0 \\
\mathbf{0}\end{array}$ & $\begin{array}{l}7 \\
* \\
7\end{array}$ \\
\hline 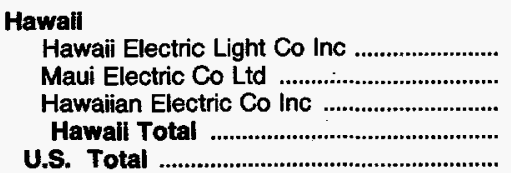 & $\begin{array}{l}\text { Investor-Owned } \\
\text { Investor-Owned } \\
\text { Investor-Owned }\end{array}$ & $\begin{array}{r}0 \\
* \\
* \\
8,851\end{array}$ & $\begin{array}{r}* \\
2 \\
3 \\
5 \\
7,541\end{array}$ & $\begin{array}{r}0 \\
0 \\
0 \\
0 \\
6,270\end{array}$ & $\begin{array}{r}0 \\
0 \\
0 \\
0 \\
407\end{array}$ & $\begin{array}{r}* \\
2 \\
3 \\
5 \\
23,069\end{array}$ \\
\hline
\end{tabular}

* Value less than 0.5

Notes: -Data are final. Data are provided for electric utilities with sales to uitimate consumers or sales for resale greater than or equal to 120,000 megawatthours. - Totals may not equal sum of components because of independent rounding. - These data reflect actual real changes in the demand for electricity at the time of annual peak load, as opposed to the installed peakload reduction capability (i.e. Potential Peakload Reduction). Source: Energy Information Administration, Form EIA-861, "Annual Electric Utility Report." 


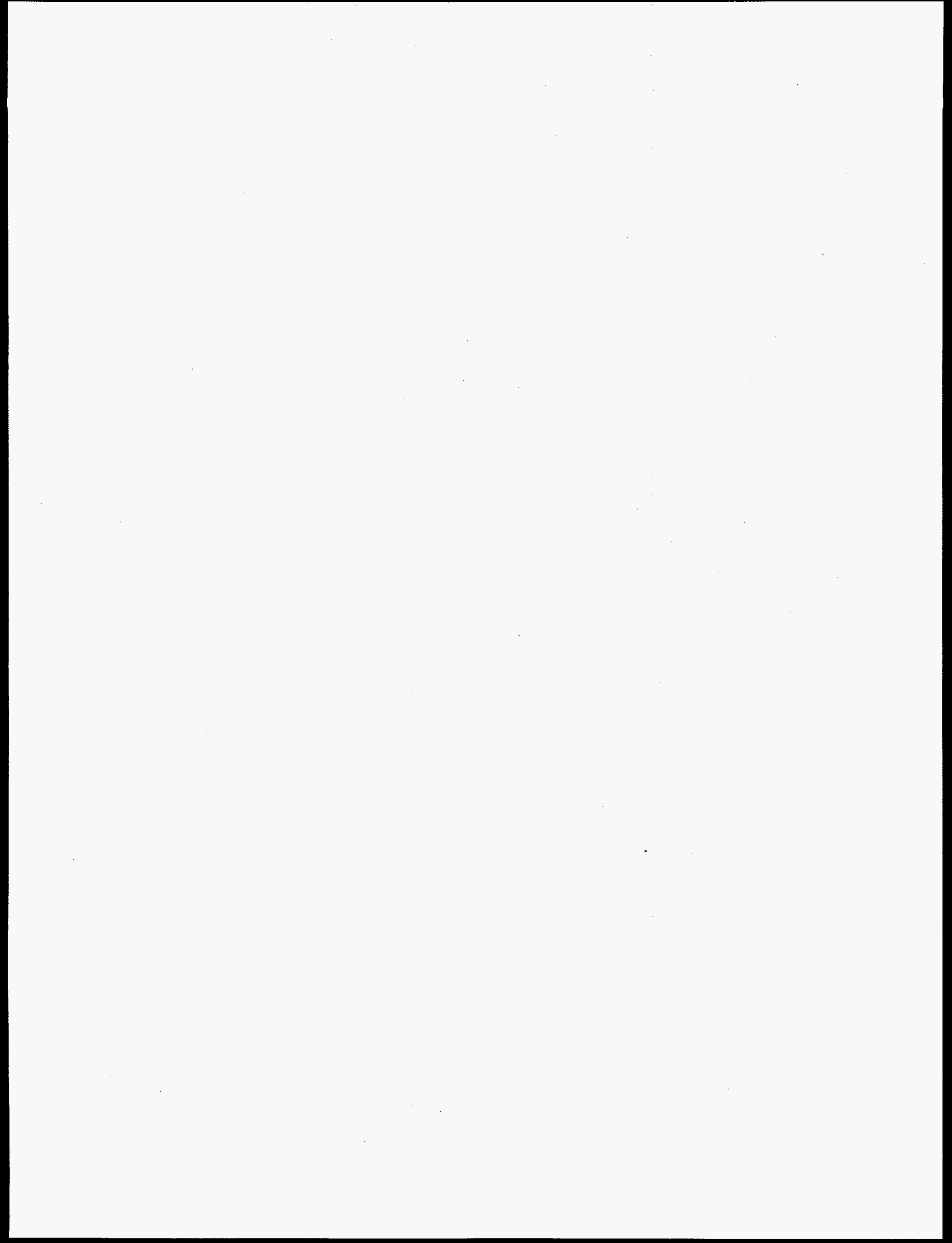




\section{Cost}

Utility costs ${ }^{13}$ for DSM programs are reported by electric utilities using two categories: direct utility costs and indirect utility costs. Direct utility costs are those directly attributable to a specific DSM program category. Indirect utility costs are those incurred by utilities that are not directly attributable to a specific DSM program category. Total utility cost is the summation of direct utility costs and indirect utility costs.

In 1993, total utility cost for large utilities with DSM programs was $\$ 2.7$ billion, an increase of 17 percent over 1992 total utility cost of $\$ 2.3$ billion. ${ }^{14}$ Since 1989 , total utility cost has increased $\$ 1.9$ billion, at an average annual rate of 33 percent. For 1994, total utility cost was predicated to increase 9 percent to $\$ 3.0$ billion and by 1998 to $\$ 3.6$ billion (Table 21 ).

The majority of utilities with DSM programs spent between 0.1 and 1 percent of electric revenues from sales to ultimate consumers on DSM programs. Among large utilities, 10 percent spent less than 0.1 percent of revenues on DSM, 49 percent spent between 0.1 and 1 percent of revenues on DSM, and 40 percent spent more than 1 percent of revenues on DSM. There were 62 cooperatives, 74 investor-owned utilities, and 43 publicly owned utilities that spent more than 1 percent of revenues on DSM. Of the utilities spending between 0.1 and $I$ percent, there were 77 publicly owned, 93 cooperatives, and 50 investorowned utilities (Figure 8).

In 1993,98 percent of total spending on DSM was made by the 100 utilities reporting the greatest DSM costs. The 50 utilities that spent the most on DSM accounted for 86 percent of the total costs, and the top 25 utilities accounted for 67 percent (Figure 9). These 100, 50, and 25 utilities that had the greatest cost for DSM programs represented 64, 46, and 29 percent, respectively, of total retail sales of electricity in the United States.
In 1993, investor-owned utilities spent the most on DSM, $\$ 2.3$ billion, followed by Federally owned utilities, ${ }^{15} \$ 238$ million, publicly owned utilities, $\$ 167$ million, and cooperatives, $\$ 88$ million. Both publicly owned and Federally owned utilities predicted 24-percent increases for 1994. For 1998, investorowned utilities predicted the greatest increase, 4 percent annually to $\$ 2.8$ billion (Table 22 ).

Direct Utility Costs are those identified specifically with one of the DSM program categories (i.e., energy efficiency, direct load control, interruptible load control, other load management, other DSM programs, or load building). In 1993, direct utility costs for large utilities was $\$ 2.3$ billion dollars. Seventy percent of direct utility costs were for energy efficiency programs, amounting to $\$ 1.6$ billion (Table 23). Direct costs reported by utilities do not include lost revenue as a result of offering customers interruptible rates.

Among the NERC regions, WSCC had the greatest share of direct utility costs, spending $\$ 653$ million, mainly because within the WSCC there were three utilities with direct utility costs over $\$ 100$ million. The second largest region was SERC with direct utility costs of $\$ 505$ million, where two utilities had nearly $\$ 100$ million of direct utility costs each.

Indirect Utility Costs are utility costs that may not be meaningfully identified with any particular DSM program category. Indirect costs could be attributable to one of several accounting cost categories (i.e., Administrative, Marketing, Monitoring and Evaluation, Utility-Earned Incentives ${ }^{16}$ or Other).$^{17}$ Indirect utility costs for 1993 were $\$ 454$ million, with the greatest portion of these costs for administrative costs and other. Among the NERC regions, SERC had the highest share of indirect utility costs, $\$ 138$ million, followed by WSCC with $\$ 104$ million (Table 24).

13 Utilities are required to report nonutility costs (nonutility costs are those incurred by the consumer, such as installation of an energy efficient appliance, or by the retailer or manufacturer of energy efficient products) but are not included in this report because in many cases it is not possible for utilities to accurately estimate this cost.

14 Small utilities are not included in this section as they report only total utility cost and not a breakdown into direct and indirect costs.

15 The large amount of spending reported by Federally owned utilities may be misleading. Both the Tennessee Valley Authority and Bonneville Power Administration encourage utilities to use DSM, and finance their programs.

16 Utility-Earned Incentives are not included in this publication.

17 Other costs include the indirect cost of DSM that cannot be attributed to any other cost category, particularly research and development. 
Figure 8. U.S. Electric Utility DSM Program Costs as a Percentage of Retail Revenue by Number of Utilities with DSM Costs, 1993



Note: This graph only shows utllities that reported DSM costs.

Source: Energy Information Administration, Form EIA-861, "Annual Electric Utility Report."

Figure 9. The Top 25, 50, and 100 U.S. Electric Utilities with the Greatest DSM Program Costs by Class of Ownership, 1993

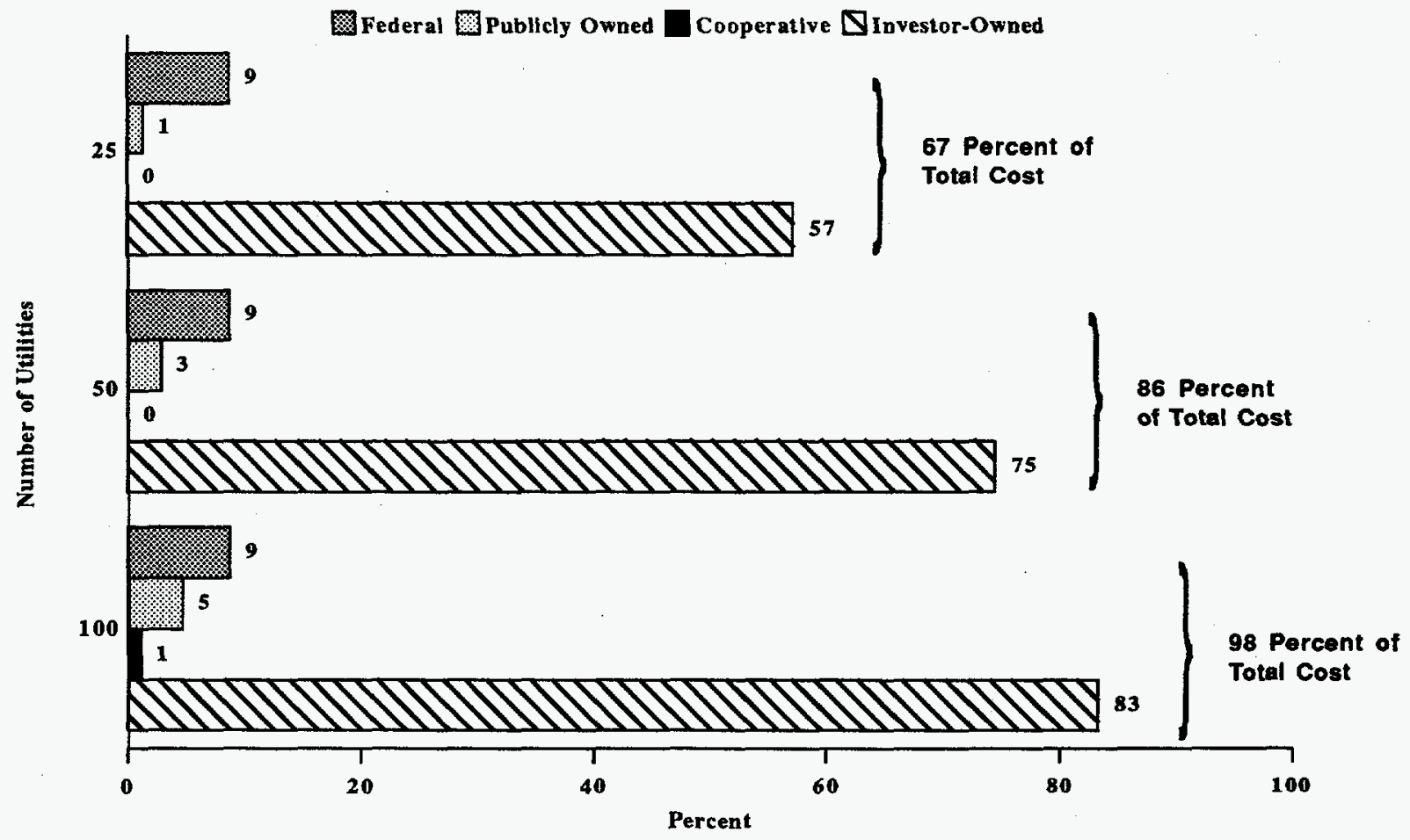

Note: There are no cooperatives included in the top 25 or $\mathbf{5 0}$ electric utlittles.

Sources: Energy Information Administration, Form EIA-861, "Annual Electric Utility Report." 
Table 21. U.S. Electric Utility DSM Program Costs by Class of Ownership, 1989 Through 1993, 1994, and 1998

(Thousand Dollars)

\begin{tabular}{|c|c|c|c|c|c|c|c|}
\hline \multirow{2}{*}{ Class of Ownership } & \multicolumn{5}{|c|}{ Historical Costs } & \multicolumn{2}{|c|}{ Projected Costs } \\
\hline & 1989 & 1990 & 1991 & 1992 & 1993 & 1994 & 1998 \\
\hline 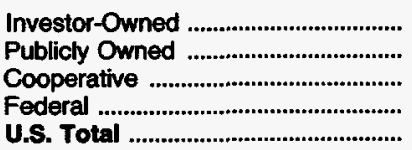 & $\begin{array}{r}782,443 \\
44,381 \\
41,463 \\
4,648 \\
872,935\end{array}$ & $\begin{array}{r}1,065,127 \\
74,475 \\
32,055 \\
5,600 \\
1,177,457\end{array}$ & $\begin{array}{r}1,509,412 \\
179,767 \\
52,954 \\
61,640 \\
1,803,773\end{array}$ & $\begin{array}{r}1,918,803 \\
163,075 \\
81,553 \\
184,663 \\
2,348,094\end{array}$ & $\begin{array}{r}2,251,227 \\
166,774 \\
87,818 \\
237,714 \\
2,743,533\end{array}$ & $\begin{array}{r}2,404,900 \\
207,616 \\
95,099 \\
295,357 \\
3,002,972\end{array}$ & $\begin{array}{r}2,786,351 \\
257,126 \\
127,504 \\
391,313 \\
3,562,294\end{array}$ \\
\hline
\end{tabular}

Notes: -Data are final. -Data are provided for electric utilities with sales to ultimate consumers or sales for resale greater than or equal to 120,000 megawatthours.

Source: Energy Information Administration, Form EIA-861, "Annual Electric Utility Report." 
Table 22. U.S. Electric Utility DSM Program Costs by North American Electric Reliability Council Region and Hawaii by Class of Ownership, 1992, 1993, 1994, and 1998

(Thousand Dollars)

\begin{tabular}{|c|c|c|c|c|c|}
\hline \multirow{2}{*}{$\begin{array}{l}\text { North American Electric Reliability } \\
\text { Council Region and Hawali / } \\
\text { Electric Utility }\end{array}$} & \multirow{2}{*}{$\begin{array}{l}\text { Class of } \\
\text { Ownership }\end{array}$} & \multicolumn{2}{|c|}{ Historical Costs } & \multicolumn{2}{|c|}{ Projected Costs } \\
\hline & & 1992 & 1993 & 1994 & 1998 \\
\hline 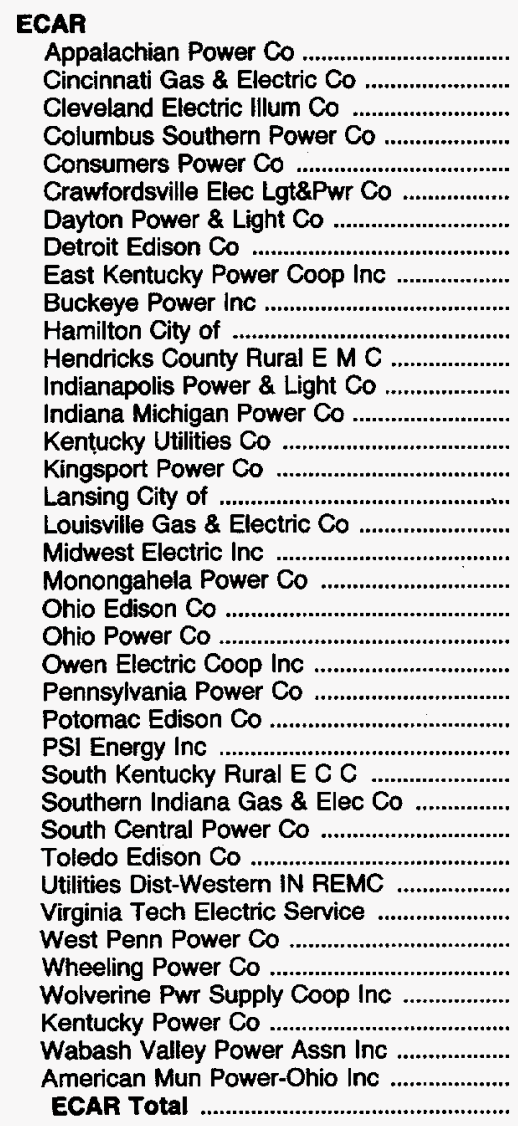 & $\begin{array}{l}\text { Investor-Owned } \\
\text { Investor-Owned } \\
\text { Investor-Owned } \\
\text { Investor-Owned } \\
\text { Investor-Owned } \\
\text { Publicly Owned } \\
\text { Investor-Owned } \\
\text { Investor-Owned } \\
\text { Cooperative } \\
\text { Cooperative } \\
\text { Publicly Owned } \\
\text { Cooperative } \\
\text { Investor-Owned } \\
\text { Investor-Owned } \\
\text { Investor-Owned } \\
\text { Investor-Owned } \\
\text { Publicly Owned } \\
\text { Investor-Owned } \\
\text { Cooperative } \\
\text { Investor-Owned } \\
\text { Investor-Owned } \\
\text { Investor-Owned } \\
\text { Cooperative } \\
\text { Investor-Owned } \\
\text { Investor-Owned } \\
\text { Investor-Owned } \\
\text { Cooperative } \\
\text { Investor-Owned } \\
\text { Cooperative } \\
\text { Investor-Owned } \\
\text { Cooperative } \\
\text { Publicly Owned } \\
\text { Investor-Owned } \\
\text { Investor-Owned } \\
\text { Cooperative } \\
\text { Investor-Owned } \\
\text { Cooperative } \\
\text { Publicly Owned }\end{array}$ & $\begin{array}{r}654 \\
1,403 \\
204 \\
1,592 \\
32,764 \\
- \\
6,699 \\
2,015 \\
1,900 \\
1,940 \\
- \\
- \\
2,300 \\
1,703 \\
2,000 \\
310 \\
- \\
3,400 \\
129 \\
333 \\
32,058 \\
2,164 \\
63 \\
3,085 \\
566 \\
18,012 \\
211 \\
3,233 \\
606 \\
574 \\
151 \\
5 \\
1,825 \\
51 \\
0 \\
133 \\
8,720 \\
100 \\
130,903\end{array}$ & $\begin{array}{r}1,473 \\
4,188 \\
3,416 \\
3,069 \\
52,566 \\
9 \\
25,353 \\
4,096 \\
1,900 \\
2,015 \\
115 \\
60 \\
2,557 \\
899 \\
2,370 \\
210 \\
26 \\
4,343 \\
161 \\
457 \\
14,851 \\
2,696 \\
96 \\
2,909 \\
1,520 \\
35,399 \\
-1 \\
5,869 \\
700 \\
1,556 \\
262 \\
10 \\
2,187 \\
103 \\
885 \\
16 \\
8,695 \\
100 \\
187,137\end{array}$ & $\begin{array}{r}3,764 \\
13,410 \\
3,340 \\
3,378 \\
14,693 \\
138 \\
17,700 \\
7,600 \\
1,900 \\
2,000 \\
135 \\
50 \\
6,239 \\
2,090 \\
3,952 \\
210 \\
63 \\
5,847 \\
170 \\
401 \\
22,215 \\
3,804 \\
114 \\
3,055 \\
16,901 \\
33,932 \\
- \\
9,737 \\
758 \\
1,520 \\
268 \\
10 \\
5,232 \\
103 \\
772 \\
464 \\
9,595 \\
105 \\
195,665\end{array}$ & $\begin{array}{r}11,206 \\
17,146 \\
9,570 \\
9,280 \\
50,000 \\
30 \\
17,700 \\
9,500 \\
1,900 \\
2,490 \\
140 \\
50 \\
10,769 \\
7,240 \\
4,817 \\
902 \\
190 \\
6,497 \\
193 \\
642 \\
12,624 \\
10,580 \\
124 \\
3,324 \\
1,182 \\
30,425 \\
11,991 \\
940 \\
4,545 \\
302 \\
10 \\
5,805 \\
558 \\
775 \\
1,957 \\
10,845 \\
180 \\
256,429\end{array}$ \\
\hline 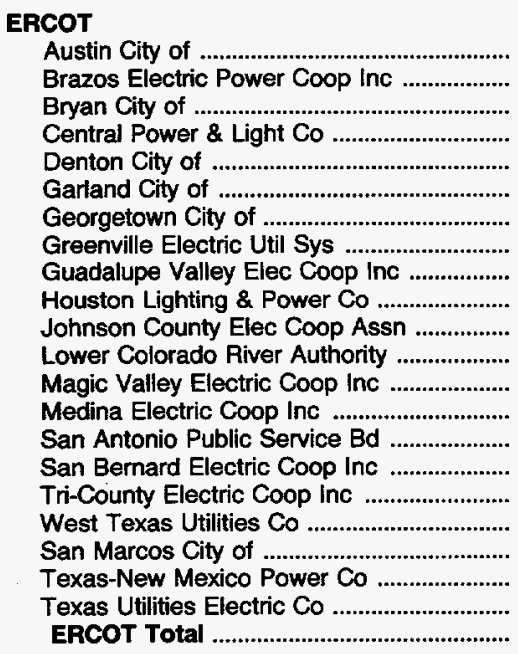 & $\begin{array}{l}\text { Publicly Owned } \\
\text { Cooperative } \\
\text { Publicly Owned } \\
\text { Investor-Owned } \\
\text { Publicly Owned } \\
\text { Publicly Owned } \\
\text { Publicly Owned } \\
\text { Publicly Owned } \\
\text { Cooperative } \\
\text { Investor-Owned } \\
\text { Cooperative } \\
\text { Publicly Owned } \\
\text { Cooperative } \\
\text { Cooperative } \\
\text { Publicly Owned } \\
\text { Cooperative } \\
\text { Cooperative } \\
\text { Investor-Owned } \\
\text { Publicly Owned } \\
\text { Investor-Owned } \\
\text { Invistor-Owned }\end{array}$ & $\begin{array}{r}4,764 \\
261 \\
269 \\
2,686 \\
350 \\
618 \\
- \\
444 \\
231 \\
17,885 \\
57 \\
3,339 \\
- \\
95 \\
385 \\
77 \\
66 \\
1,562 \\
64 \\
1,013 \\
21,509 \\
\mathbf{5 5 , 6 7 5}\end{array}$ & $\begin{array}{r}9,839 \\
779 \\
366 \\
6,596 \\
268 \\
626 \\
36 \\
19 \\
158 \\
17,922 \\
119 \\
3,311 \\
88 \\
86 \\
0 \\
59 \\
52 \\
990 \\
77 \\
1,040 \\
20,102 \\
62,533\end{array}$ & $\begin{array}{r}11,299 \\
1,015 \\
480 \\
4,907 \\
493 \\
614 \\
40 \\
51 \\
288 \\
21,372 \\
138 \\
3,284 \\
167 \\
82 \\
1,460 \\
71 \\
73 \\
1,655 \\
82 \\
1,570 \\
16,415 \\
65,556\end{array}$ & $\begin{array}{r}13,794 \\
3,975 \\
800 \\
8,602 \\
1,016 \\
577 \\
43 \\
190 \\
338 \\
21,372 \\
140 \\
5,230 \\
380 \\
92 \\
1,877 \\
73 \\
125 \\
2,275 \\
92 \\
1,750 \\
16,415 \\
79,156\end{array}$ \\
\hline 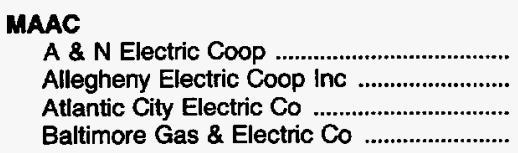 & $\begin{array}{l}\text { Cooperative } \\
\text { Cooperative } \\
\text { Investor-Owned } \\
\text { Investor-Owned }\end{array}$ & $\begin{array}{r}140 \\
217 \\
10,934 \\
29,791\end{array}$ & $\begin{array}{r}145 \\
10,397 \\
65,683\end{array}$ & $\begin{array}{r}150 \\
7,546 \\
71,989\end{array}$ & $\begin{array}{r}168 \\
- \\
6,703 \\
60,259\end{array}$ \\
\hline
\end{tabular}

See footnotes at end of table. 
Table 22. U.S. Electric Utility DSM Program Costs by North American Electric Reliability Council Region and Hawaii by Class of Ownership, 1992, 1993, 1994, and 1998 (Thousand Dollars) (Continued)

\begin{tabular}{|c|c|c|c|c|c|}
\hline \multirow{2}{*}{$\begin{array}{l}\text { North American Electric Rellability } \\
\text { Council Region and Hawall / } \\
\text { Electric Utility }\end{array}$} & \multirow{2}{*}{$\begin{array}{l}\text { Class of } \\
\text { Ownership }\end{array}$} & \multicolumn{2}{|c|}{ Historical Costs } & \multicolumn{2}{|c|}{ Projected Costs } \\
\hline & & 1992 & 1993 & 1994 & 1998 \\
\hline
\end{tabular}

MAC (Continued)

Choptank Electric Coop Inc .........................

Conowingo Power Co ....

Delmarva Power \& Light Co

Delaware Electric Coop Inc

Easton Utilities Comm ....................................

Jersey Central Power\&Light Co ...................

Metropolitan Edison Co

Pennsylvania Electric Co ..............................

Pennsylvania Power \& Light Co ...................

Philadelphia Electric Co

Potomac Electric Power Co ..........................

Public Service Electric\&Gas $\mathrm{C}$

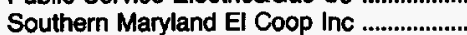

UGI Utilities Inc

Somerset Rural Elec Coop Inc ....................

Adams Electric Coop Inc

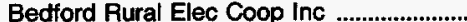

Valley Rural Electric Coop Inc .....................

Central Electric Coop Inc.

Southwest Central R E C Corp .........

MAAC Total

MAIN

Boone Electric Coop

Central Illinois Light $\mathrm{Co}_{0}$

Central Illinois Pub Serv Co

Coles-Moultrie Electric COOp

Columbia City of .

Commonwealth Edison $\mathrm{Co}$

Corn Belt Electric Coop Inc

Cuivre River Electric Coop Inc.

Eastern Illini Electric Coop .

Illinois Power Co

Madison Gas \& Electric Co

Manitowoc Public Utilities ..............................

Marshfield City of ...

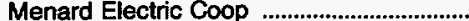

Plymouth City of ...

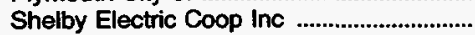

South Beloit Water Gas\&Elec Co

Southeastern IL Elec Coop Inc .................

Southwestern Electric Coop Inc ..................

Springfield City of

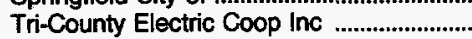

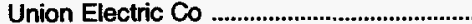

Wayne-White Counties Elec Coop ..............

Wisconsin Electric Power Co

Wisconsin Power \& Light Co .

Wisconsin Public Power Inc Sys

Wisconsin Public Service Corp

MAIN Total

MAPP(U.S.)

Ames City of

Anoka Electric Coop ......................................

Anoka City of

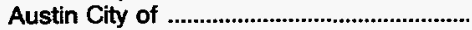

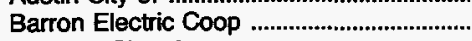

Beatrice City of

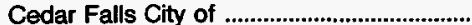

Central lowa Power Coop ...............................

Central Power Elec Coop inc

Clark Electric Coop

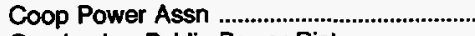

Comhusker Public Power Dist

Dawson County Public Pwr Dist
Cooperative

Investor-Owned

Investor-Owned

Cooperative

Publicly Owned

Investor-Owned

Investor-Owned

Investor-Owned

Investor-Owned

Investor-Owned

Investor-Owned

Investor-Owned

Cooperative

Investor-Owned

Cooperative

Cooperative

Cooperative

Cooperative

Cooperative

Cooperative

Cooperative

Cooperative

Cooperative

Investor-Owned

Investor-Owned

Cooperative

Publicly Owned

Investor-Owned

Cooperative

Cooperative

Cooperative

investor-Owned

Investor-Owned

Publicly Owned

Publicly Owned

Cooperative

Publicly Owned

Cooperative

Investor-Owned

Cooperative

Cooperative

Publicly Owned

Cooperative

Investor-Owned

Cooperative

Investor-Owned

investor-Owned

Publicly Owned

Investor-Owned

Publicly Owned

Cooperative

Publicly Owned

Publicly Owned

Cooperative

Publicly Owned

Publicly Owned

Cooperative

Cooperative

Cooperative

Cooperative

Publicly Owned

Publicly Owned
185
179

8,820

732

$8,8 \overline{45}$

4,705

3,368

12,310

9,983

40,705

43,570

2,998

79

116

196

100

109

$-$

323

178,420

118

2,437
819

180

303

3,110

231

71

80
460

7,080

310

138

84

$-\overline{145}$

75

3
747

333

550

19,200

22

54,005

$\mathbf{9 , 4 8 6}$

32,635

133,610

120

1,193

$1 \overline{17}$

117

41

253

82

.55

5,218

30

38

229

429

8,902
662

49

13,685

4,461

3,376

13,050

10,606

73,516

50,200

5,173

97

123

519

106

106

132

101

262,111

265

1,736

5,821

750

344
17.805

17,805
4,326

4,326
3,967

13,277

10,190

96,796

58,658

6,947

110

130

568

108

111

167

105
30

30

302,236

371

1,736

1,000

534

13,737

4,326

4,452

13,192

19,997

94,469

145,000

10,665

110

146

689

120

127

232
118

50

367

$\mathbf{3 8 8 , 0 9 8}$

82

3,140
691

691

130

744

8,300

292

168

105

6,782

450

172
79

15

70

11
180

180
527

115

13,930

25

48,219

14,716

2,332

25,000

126,275

91

5,252

691
115

1,200
95,220

330

167

82

7,415

500

172

88

15

37 
Table 22. U.S. Electric Utility DSM Program Costs by North American Electric Reliability Council Region and Hawail by Class of Ownership, 1992, 1993, 1994, and 1998 (Thousand Dollars) (Continued)

\begin{tabular}{|c|c|c|c|c|c|}
\hline \multirow{2}{*}{$\begin{array}{c}\text { North American Electric Rellability } \\
\text { Council Region and Hawall / } \\
\text { Eloctric Utility }\end{array}$} & \multirow{2}{*}{$\begin{array}{l}\text { Class of } \\
\text { Ownership }\end{array}$} & \multicolumn{2}{|c|}{ Historical Costs } & \multicolumn{2}{|c|}{ Projected Costs } \\
\hline & & 1992 & 1993 & 1994 & 1998 \\
\hline 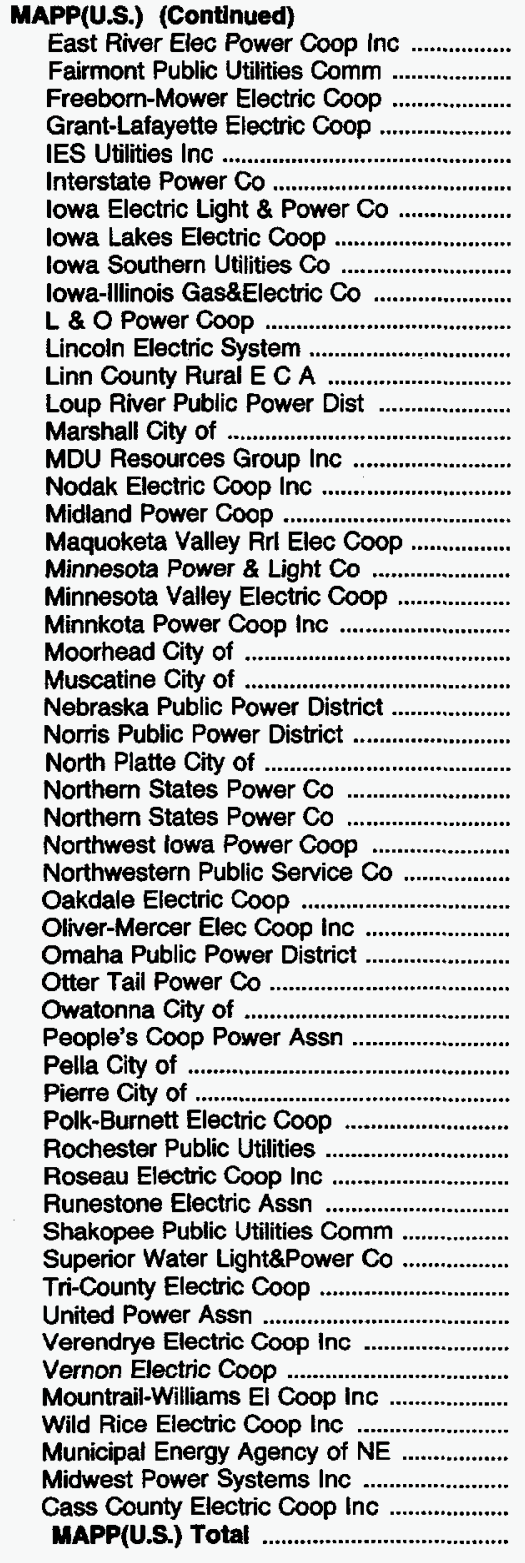 & $\begin{array}{l}\text { Cooperative } \\
\text { Publicly Owned } \\
\text { Cooperative } \\
\text { Cooperative } \\
\text { Investor-Owned } \\
\text { Investor-Owned } \\
\text { Investor-Owned } \\
\text { Cooperative } \\
\text { Investor-Owned } \\
\text { Investor-Owned } \\
\text { Cooperative } \\
\text { Publicly Owned } \\
\text { Cooperative } \\
\text { Publicly Owned } \\
\text { Publicly Owned } \\
\text { Investor-Owned } \\
\text { Cooperative } \\
\text { Cooperative } \\
\text { Cooperative } \\
\text { Investor-Owned } \\
\text { Cooperative } \\
\text { Cooperative } \\
\text { Publicly Owned } \\
\text { Publicly Owned } \\
\text { Publicly Owned } \\
\text { Publicly Owned } \\
\text { Publicly Owned } \\
\text { Investor-Owned } \\
\text { Investor-Owned } \\
\text { Cooperative } \\
\text { Investor-Owned } \\
\text { Cooperative } \\
\text { Cooperative } \\
\text { Publicly Owned } \\
\text { Investor-Owned } \\
\text { Publicly Owned } \\
\text { Cooperative } \\
\text { Publicly Owned } \\
\text { Publicly Owned } \\
\text { Cooperative } \\
\text { Publicly Owned } \\
\text { Cooperative } \\
\text { Cooperative } \\
\text { Publicly Owned } \\
\text { Investor-Owned } \\
\text { Cooperative } \\
\text { Cooperative } \\
\text { Cooperative } \\
\text { Cooperative } \\
\text { Cooperative } \\
\text { Cooperative } \\
\text { Publicly Owned } \\
\text { Investor-Owned } \\
\text { Cooperative }\end{array}$ & 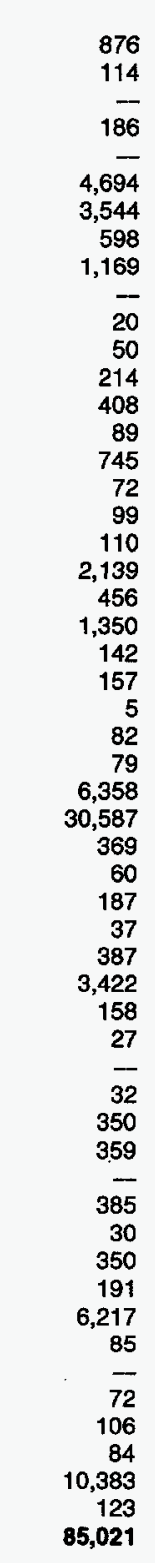 & $\begin{array}{r}2,479 \\
268 \\
59 \\
107 \\
5,436 \\
4,892 \\
- \\
622 \\
- \\
1,502 \\
20 \\
77 \\
- \\
- \\
216 \\
900 \\
66 \\
107 \\
- \\
3,437 \\
- \\
2,123 \\
132 \\
247 \\
1,108 \\
58 \\
86 \\
6,305 \\
34,291 \\
386 \\
6 \\
102,895 \\
125 \\
176 \\
15 \\
459 \\
3,664 \\
62 \\
26 \\
215 \\
134 \\
336 \\
128 \\
442 \\
65 \\
111 \\
30 \\
261 \\
189 \\
4,679 \\
85 \\
\end{array}$ & $\begin{array}{r}2,236 \\
99 \\
73 \\
119 \\
9,131 \\
5,210 \\
- \\
517 \\
- \\
1,706 \\
20 \\
82 \\
- \\
- \\
99 \\
875 \\
66 \\
109 \\
- \\
5,614 \\
- \\
2,238 \\
145 \\
273 \\
1,210 \\
150 \\
110 \\
7,435 \\
49,989 \\
401 \\
2 \\
128,766\end{array}$ & 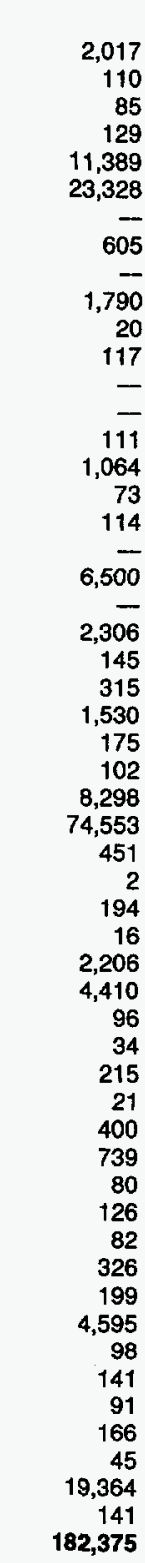 \\
\hline 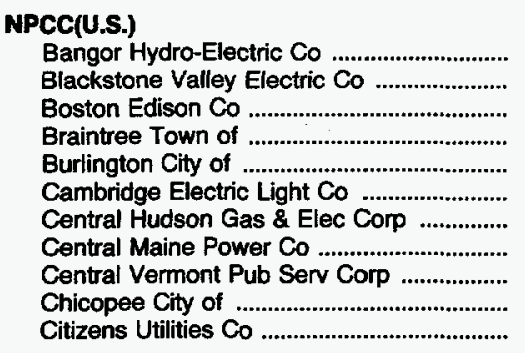 & $\begin{array}{l}\text { Investor-Owned } \\
\text { investor-Owned } \\
\text { Investor-Owned } \\
\text { Publicly Owned } \\
\text { Publicly Owned } \\
\text { Investor-Owned } \\
\text { Investor-Owned } \\
\text { Investor-Owned } \\
\text { Investor-Owned } \\
\text { Publicly Owned } \\
\text { Investor-Owned }\end{array}$ & $\begin{array}{r}2,116 \\
1,948 \\
54,871 \\
180 \\
883 \\
579 \\
8,834 \\
17,256 \\
4,169 \\
261 \\
105\end{array}$ & $\begin{array}{r}1,989 \\
3,100 \\
55,823 \\
129 \\
954 \\
2,686 \\
5,011 \\
15,350 \\
9,841 \\
175 \\
0\end{array}$ & $\begin{array}{r}1,613 \\
3,295 \\
57,453 \\
200 \\
584 \\
1,500 \\
7,632 \\
17,500 \\
5,550 \\
390 \\
285\end{array}$ & $\begin{array}{r}1,613 \\
2,959 \\
32,513 \\
168 \\
584 \\
1,500 \\
11,630 \\
17,500 \\
2,826 \\
730 \\
2,134\end{array}$ \\
\hline
\end{tabular}

See footnotes at end of table. 
Table 22. U.S. Electric Utility DSM Program Costs by North American Electric Reliability Council Region and Hawaii by Class of Ownership, 1992, 1993, 1994, and 1998 (Thousand Dollars) (Continued)

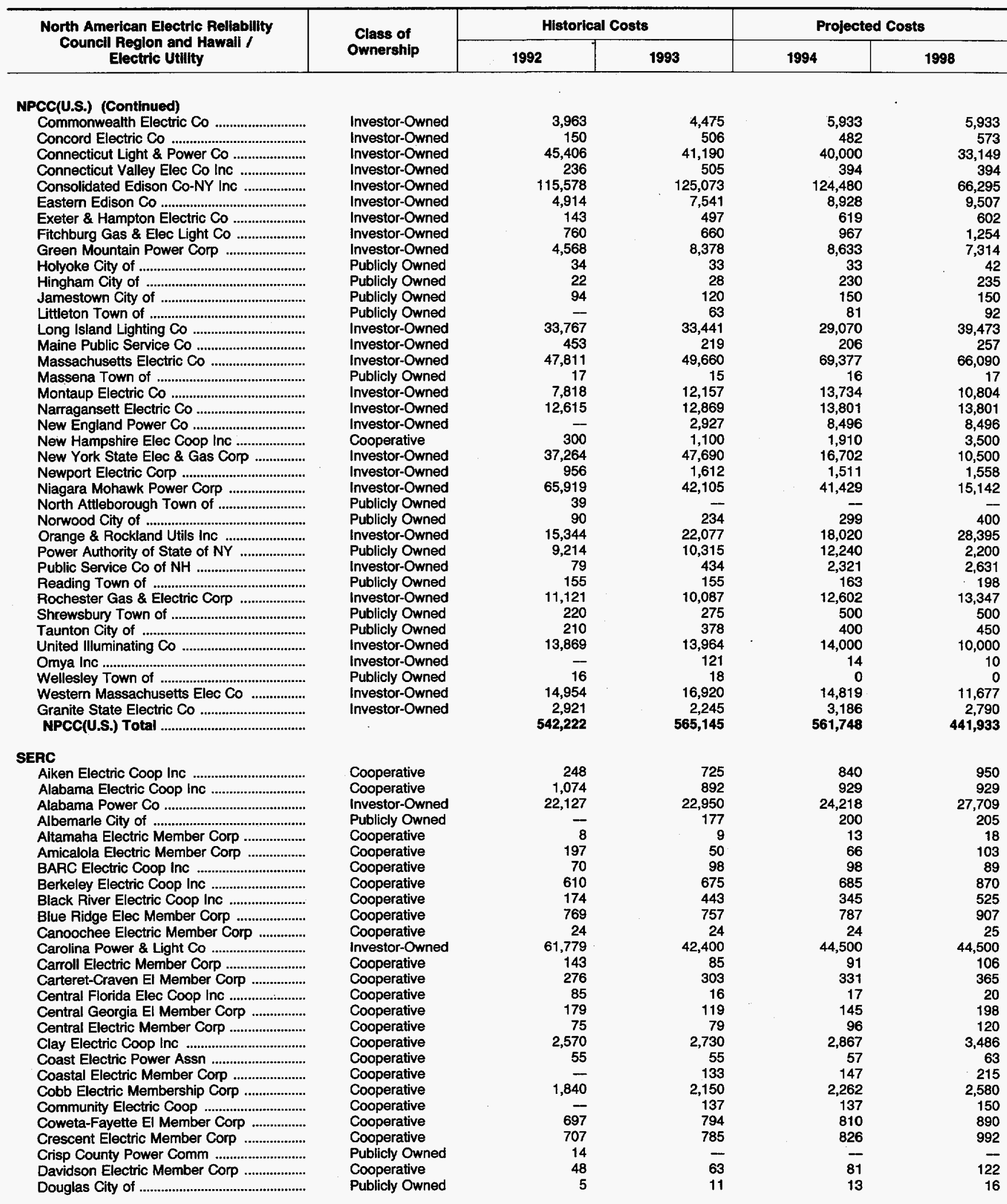

See footnotes at end of table. 
Table 22. U.S. Electric Utility DSM Program Costs by North American Electric Reliability Council Region and Hawaii by Class of Ownership, 1992, 1993, 1994, and 1998 (Thousand Dollars) (Continued)

\begin{tabular}{|c|c|c|c|c|c|}
\hline \multirow{2}{*}{$\begin{array}{c}\text { North American Electric Reliabillty } \\
\text { Council Region and Hawall / } \\
\text { Electric Utility }\end{array}$} & \multirow{2}{*}{$\begin{array}{l}\text { Class of } \\
\text { Ownershlp }\end{array}$} & \multicolumn{2}{|c|}{ Historical Costs } & \multicolumn{2}{|c|}{ Projected Costs } \\
\hline & & 1992 & 1993 & 1994 & 1998 \\
\hline \multicolumn{6}{|l|}{ SERC (Continued) } \\
\hline 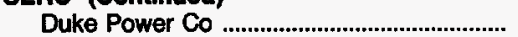 & Investor-Owned & 75,031 & 86,241 & 80,429 & 111,469 \\
\hline 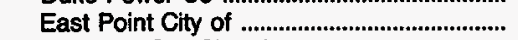 & Publicly Owned & - & 0 & 18 & 22 \\
\hline 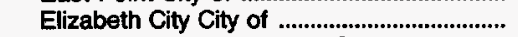 & Publicly Owned & 307 & 288 & 288 & 288 \\
\hline Excelsior Electric Member Corp ..................... & Cooperative & 18 & 45 & 20 & 30 \\
\hline 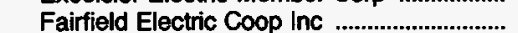 & Cooperative & 169 & 168 & 137 & 108 \\
\hline 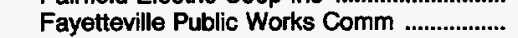 & Publicly Owned & 25 & 25 & 200 & 25 \\
\hline Fitzgerald Wtr Lgt \& Bond Comm ................ & Publicly Owned & 19 & 7 & 10 & 10 \\
\hline Flint Electric Membership Corp .................... & Cooperative. & 1,532 & 1,729 & 1,794 & 1,968 \\
\hline 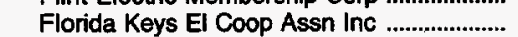 & Cooperative & 166 & 189 & 183 & 205 \\
\hline 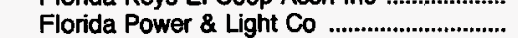 & Investor-Owned & 95,841 & 139,381 & 154,853 & 183,662 \\
\hline Florida Power Corp & Investor-Owned & 76,634 & 102,930 & 105,190 & 105,190 \\
\hline Fort Pierce Utilities Auth ................................ & Publicly Owned & 135 & 163 & 175 & 175 \\
\hline Four County Elec Member Corp ................... & Cooperative & 257 & 1,083 & 1,158 & 1,359 \\
\hline 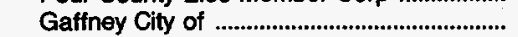 & Publicly Owned & - & 1,802 & 961 & 268 \\
\hline Gainesville Regional Utilities ........................ & Publicly Owned & 603 & 510 & 514 & 602 \\
\hline Gastonia City of & Publicly Owned & 50 & - & - & - \\
\hline 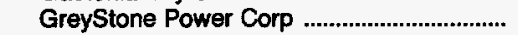 & Cooperative & 60 & 353 & 285 & 238 \\
\hline Georgla Power Co & Investor-Owned & 6,743 & 52,294 & 58,884 & 62,944 \\
\hline Grady County Elec Member Corp ............... & Cooperative & 64 & 27 & 27 & 31 \\
\hline Greenville Utilities Comm .............................. & Publicly Owned & 481 & 400 & 501 & 596 \\
\hline 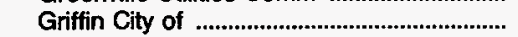 & Publicly Owned & - & 25 & 25 & 25 \\
\hline 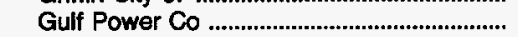 & Investor-Owned & 2,567 & 1,958 & 2,304 & 2,850 \\
\hline Harrisonburg City of ........................................ & Publicly Owned & 111 & 47 & 47 & 47 \\
\hline 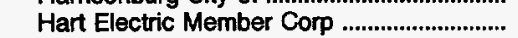 & Cooperative & 210 & 238 & 240 & 235 \\
\hline Haywood Electric Member Corp ................... & Cooperative & 42 & 43 & 43 & 58 \\
\hline 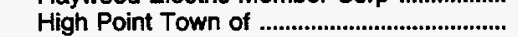 & Publicly Owned & 363 & 211 & 219 & 225 \\
\hline Jackson Electric Member Corp ..................... & Cooperative & 423 & 454 & 477 & 582 \\
\hline 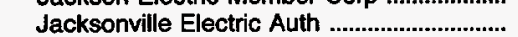 & Publicly Owned & 800 & 995 & 995 & 1,025 \\
\hline 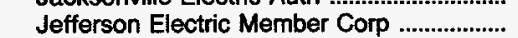 & Cooperative & 43 & 47 & 51 & $\begin{array}{r}1,0<0 \\
61\end{array}$ \\
\hline  & Cooperative & 95 & 210 & 250 & 500 \\
\hline  & Publicly Owned & 95 & 98 & 85 & 85 \\
\hline Kissimmee Utility Authority ........................ & Publicly Owned & 621 & 1,075 & 1,336 & 2,159 \\
\hline Lakeland City of & Publicly Owned & 1,949 & 1,065 & $\begin{array}{l}1,030 \\
1,358\end{array}$ & $\begin{array}{l}2,159 \\
1,523\end{array}$ \\
\hline 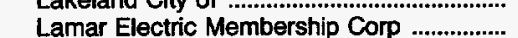 & Cooperative & $\begin{array}{r}1,040 \\
3\end{array}$ & 3 & $\begin{array}{r}1,300 \\
3\end{array}$ & $\begin{array}{r}1,023 \\
4\end{array}$ \\
\hline 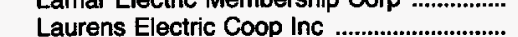 & Cooperative & 27 & 32 & 35 & $\begin{array}{r}4 \\
38\end{array}$ \\
\hline Laurinburg City of & Publicly Owned & - & 54 & 56 & 30 \\
\hline 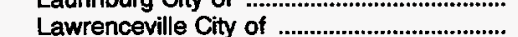 & Publicly Owned & 6 & 6 & 7 & $\begin{array}{r}39 \\
7\end{array}$ \\
\hline 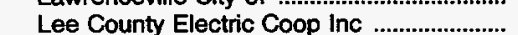 & Cooperative & 3,259 & 1.807 & 1.814 & 1963 \\
\hline 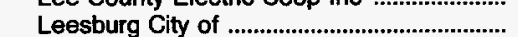 & Publicly Owned & 112 & 67 & 100 & 162 \\
\hline 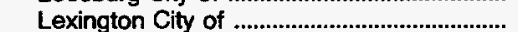 & Publicly Owned & 89 & - & - & - \\
\hline Lumbee River Elec Member Corp ................ & Cooperative & 225 & 230 & 235 & 245 \\
\hline Lumberton City of & Publicly Owned & 1 & 1 & 1 & 36 \\
\hline 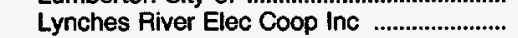 & Cooperative & 60 & 183 & 209 & 179 \\
\hline Manassas City of & Publicly Owned & 94 & 205 & 208 & 231 \\
\hline 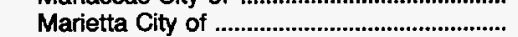 & Publicly Owned & 43 & 39 & 5 & 5 \\
\hline Mecklenburg Electric Coop inc ..................... & Cooperative & 84 & 118 & 154 & 248 \\
\hline Mid-Carolina Electric Coop Inc ..................... & Cooperative & 1,533 & 1,290 & 1,365 & 1,516 \\
\hline Mississippi Power Co & Investor-Owned & - & 106 & 109 & 119 \\
\hline Mitchell Electric Member Corp .................... & Cooperative & 28 & 28 & 28 & 35 \\
\hline 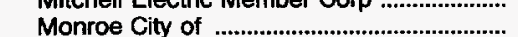 & Publicly Owned & 25 & 540 & 330 & 80 \\
\hline Morganton City of & Publicly Owned & 651 & - & - & - \\
\hline Municipal Electric Authority & Publicly Owned & 0 & 14 & $\overline{12}$ & $\overline{12}$ \\
\hline 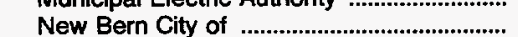 & Publicly Owned & 156 & 258 & 405 & 201 \\
\hline New River Light \& Power Co ..................... & Publicly Owned & 30 & 32 & 33 & 36 \\
\hline New Smyrna Beach Utils Comm ................... & Publicly Owned & 82 & 62 & 28 & 30 \\
\hline North Carolina Mun Power Agny .................. & Publicly Owned & 1,558 & 1,547 & 1,281 & 1,513 \\
\hline Northern Virginia Elec Coop .......................... & Cooperative & 2,015 & 2,229 & 2,298 & 7,631 \\
\hline North Carolina EI Member Corp ................... & Cooperative & 5,591 & 4,375 & 4,833 & 7,924 \\
\hline North Carolina Eastern M P A ...................... & Publicly Owned & 1,041 & 1,741 & 1,804 & 2,097 \\
\hline Northern Neck Elec Coop inc ....................... & Cooperative & 22 & 20 & 20 & 18 \\
\hline 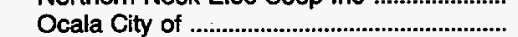 & Publicly Owned & 329 & 180 & 297 & 339 \\
\hline 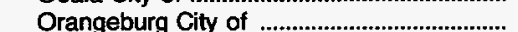 & Publicly Owned & 88 & 0 & 0 & 100 \\
\hline Palmetto Electric Coop Inc ......................... & Cooperative & 648 & 661 & 719 & 719 \\
\hline Orlando Utilities Comm & Publicly Owned & 1,548 & 1,141 & 1,866 & 3,333 \\
\hline 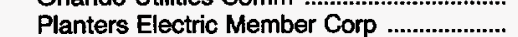 & Cooperative & 36 & 46 & 21 & $\begin{array}{r}0,000 \\
31\end{array}$ \\
\hline 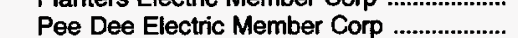 & Cooperative & 194 & 194 & 202 & 210 \\
\hline 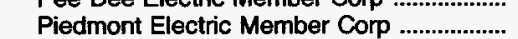 & Cooperative & 98 & 104 & 104 & 104 \\
\hline 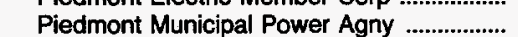 & Publicly Owned & 638 & - & - & - \\
\hline
\end{tabular}

See footnotes at end of table. 
Table 22. U.S. Electric Utility DSM Program Costs by North American Electric Reliability Council Region and Hawaii by Class of Ownership, 1992, 1993, 1994, and 1998 (Thousand Dollars) (Continued)

\begin{tabular}{c|c|c|c|c|c|c|}
\hline $\begin{array}{c}\text { North American Electric Reliability } \\
\text { Councll Region and Hawali / } \\
\text { Electric Utility }\end{array}$ & $\begin{array}{c}\text { Class of } \\
\text { Ownership }\end{array}$ & \multicolumn{2}{|c|}{ Historical Costs } & \multicolumn{2}{|c|}{ Projected Costs } \\
\cline { 3 - 6 } & & 1992 & 1993 & 1994 & 1998 & \\
\hline
\end{tabular}

SERC (Continued)

Prince George Electric Coop ......................

Randolph Electric Member Corp

Rayle Electric Membership Corp

Reedy Creek Improvement Dist

Roanoke Electric Member Corp ..................

Rock Hill City of



Rutherford Elec Member Corp ......................

Satilla Rural Elec Member Corp

Savannah Electric \& Power Co

Sawnee Electric Members Corp ..................

Shelby City of

Shenandoah Valley Elec Coop

Singing River Eleo Power Assn

South Carolina Electric\&Gas Co

Auth ........................

Snapping Shoals El Member Corp ..............

Statesville City of

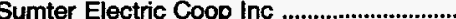

Suwannee Valley Elec Coop Inc

Tallahassee City of

Tampa Electric Co

Thomasville City of

Member Corp ....................

Tideland Electric Member Corp

Troup Electric Members Corp ...

Virginia Electric \& Power Co

Corp .......................

Walton Electric Member Corp

Washington City of

Washington Elec Member Corp

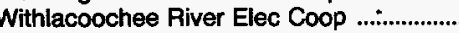

York Electric Coop Inc

Southside Electric Coop Inc .........................

Brunswick Electric Member Corp ...............

Colquitt Electric Members Corp

Central Electric Pwr Coop Inc .

Alabama Munic

SERC Total

SPP



Bailey County Elec Coop Assn .....................

C \& L Electric Coop Corp ................................

Carroll Electric Coop Corp .............................

Central Rural Electric Coop

Caddo Electric Coop Inc ........

Graighead Electric Coop Corp

Dixie Electric Membership Corp

Duncan City of

Empire District Electric Co .............................

First Electric Coop Corp

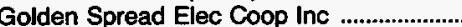

Gulf States Utilities $\mathrm{Co}$

Independence City of

Indian Electric Coop Inc

Kansas Electric Power Coop Inc ............

Kansas City Power \& Light Co ....................

KG\&E Western Resources Co

Mississippi Cnty Elec Coop Inc

North Arkansas Elec Coop Ino

Northeast Louisiana Power Coop
Cooperative

Cooperative

Cooperative

Publicly Owned

Cooperative

Publicly Owned

Publicly Owned

Cooperative

Cooperative

Investor-Owned

Cooperative

Publicly Owned

Cooperative

Cooperative

Investor-Owned

Publicly Owned

Cooperative

Cooperative

Publicly Owned

Cooperative

Cooperative

Publicly Owned

Investor-Owned

Federal

Publicly Owned

Cooperative

Cooperative

Cooperative

Cooperative

Cooperative

Investor-Owned

Cooperative

Cooperative

Publicly Owned

Cooperative

Cooperative

Cooperative

Cooperative

Cooperative

Cooperative

Cooperative

Cooperative

Publicly Owned

Investor-Owned

Cooperative

Cooperative

Cooperative

Cooperative

Cooperative

Cooperative

Cooperative

Cooperative

Publicly Owned

Investor-Owned

Cooperative

Cooperative

Investor-Owned

Publicly Owned

Cooperative

Cooperative

Investor-Owned

Investor-Owned

Cooperative

Cooperative

Cooperative

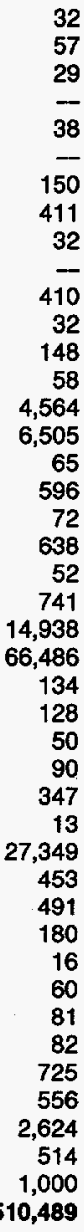

32

29

38

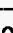

11

32

410

32

58

4,564

6,505

65

596
72

638

52

4,938

66,486

134

128
50

90

347

7,349

453

491
180

16

81

725

556

514

1,000
510,489

164

5

90

109

372

363

362

104
50

361
860

60

2,700

145

50
79

880
1,099
33

33

263

21

52

29

92

35

837

1,125

416

32

3,039

547

119

80

10,211

8,962

84
845

$7 \overline{726}$

107

951

15,923

72,445

52

210

95

117

15
398

32,398

467

491

247

16

59
45

62

714

604

2,184

553

643,081

27

27
55

30

143
39

180

4,125

437

337
32
3,039

465

137

137
90

7,004

10,487

$\begin{array}{r}78 \\ 854 \\ \hline\end{array}$

291

61

747

17,951

79,280

72

231

99

117

18
39,962

9,962

495

735

250
17

268

60

54

753

688

1,937

575

$684, \overline{107}$

42

- . 14

44

44
152

46

180

4,125

532

41

3,039

563

158

102
3,969

3,969

100

1,020

337

72
747

747
18,917

18,917
108,300

83

299

114 
Table 22. U.S. Electric Utility DSM Program Costs by North American Electric Reliability Council Region and Hawali by Class of Ownership, 1992, 1993, 1994, and 1998

(Thousand Dollars) (Continued)

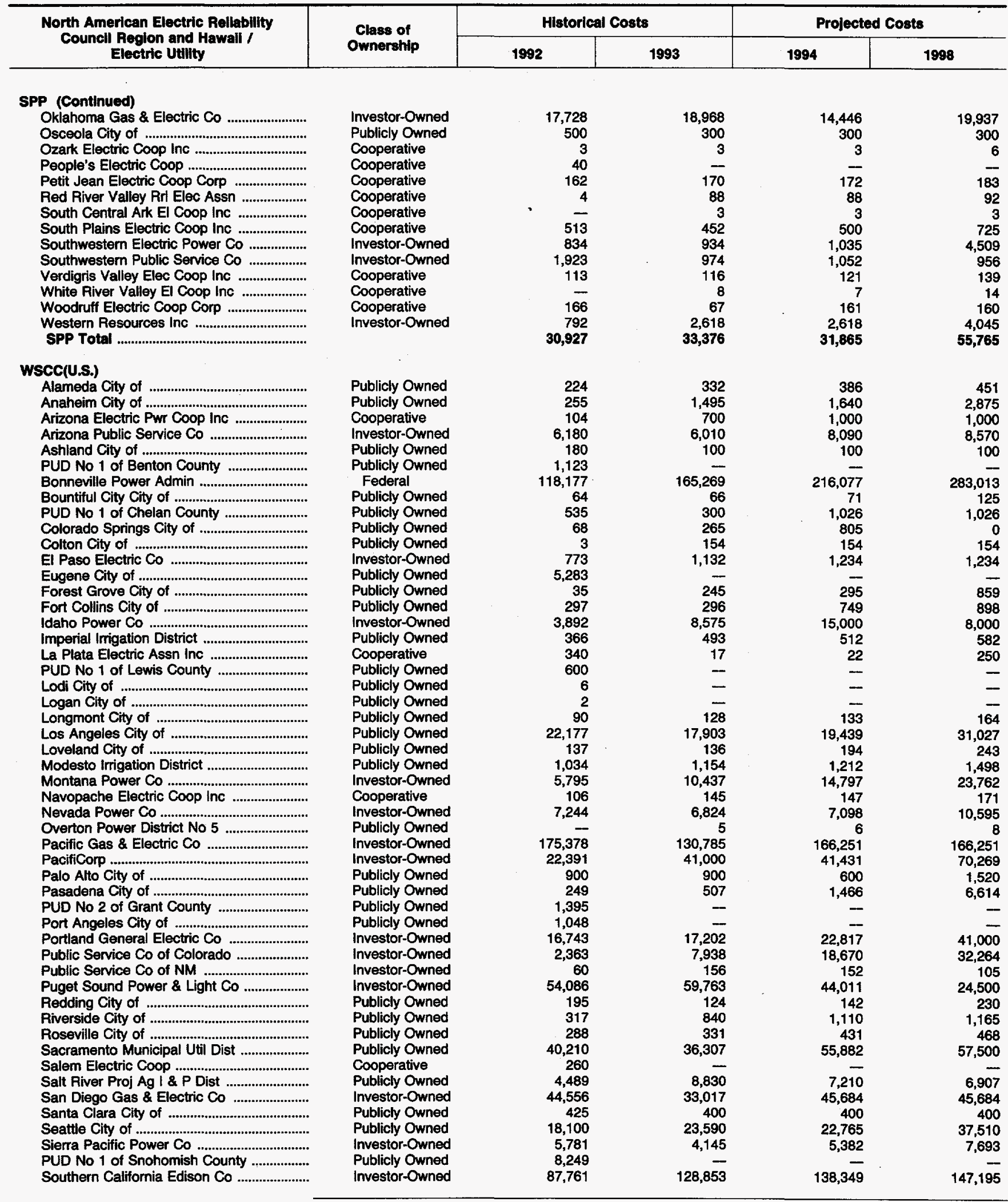

See footnotes at end of table. 
Table 22. U.S. Electric Utility DSM Program Costs by North American Electric Reliability Council Region and Hawaii by Class of Ownership, 1992, 1993, 1994, and 1998 (Thousand Dollars) (Continued)

\begin{tabular}{|c|c|c|c|c|c|}
\hline \multirow{2}{*}{$\begin{array}{l}\text { North American Electric Rellablity } \\
\text { Council Reglon and Hawall / } \\
\text { Electric Utility }\end{array}$} & \multirow{2}{*}{$\begin{array}{l}\text { Class of } \\
\text { Ownership }\end{array}$} & \multicolumn{2}{|c|}{ Historical Costs } & \multicolumn{2}{|c|}{ Projected Costs } \\
\hline & & 1992 & 1993 & 1994 & 1998 \\
\hline 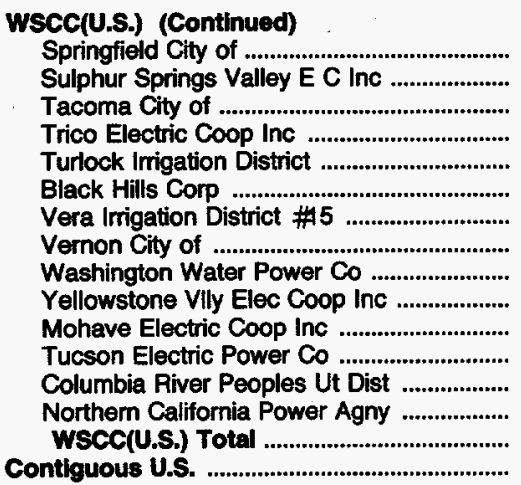 & $\begin{array}{l}\text { Publicly Owned } \\
\text { Cooperative } \\
\text { Publicly Owned } \\
\text { Cooperative } \\
\text { Publicly Owned } \\
\text { Investor-Owned } \\
\text { Publicly Owned } \\
\text { Publicly Owned } \\
\text { Investor-Owned } \\
\text { Cooperative } \\
\text { Cooperative } \\
\text { Investor-Owned } \\
\text { Publicly Owned } \\
\text { Publicly Owned }\end{array}$ & $\begin{array}{r}811 \\
110 \\
3,996 \\
- \\
451 \\
440 \\
10 \\
230 \\
10,740 \\
120 \\
10 \\
1,465 \\
370 \\
665 \\
679,752 \\
2,347,019\end{array}$ & $\begin{array}{r}1,670 \\
140 \\
8,430 \\
10 \\
894 \\
441 \\
40 \\
155 \\
25,274 \\
164 \\
20 \\
2,840 \\
- \\
- \\
756,947 \\
2,741,832\end{array}$ & $\begin{array}{r}1,688 \\
95 \\
15,300 \\
0 \\
702 \\
447 \\
40 \\
131 \\
19,456 \\
179 \\
22 \\
3,999 \\
- \\
- \\
904,999 \\
3,001,217\end{array}$ & $\begin{array}{r}2,878 \\
400 \\
17,766 \\
0 \\
830 \\
473 \\
52 \\
390 \\
6,966 \\
314 \\
42 \\
4,329 \\
- \\
- \\
1,058,320 \\
3,524,273\end{array}$ \\
\hline 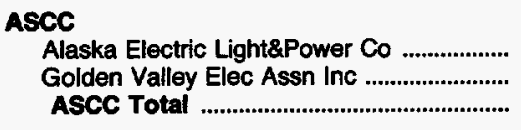 & $\begin{array}{l}\text { Investor-Owned } \\
\text { Cooperative }\end{array}$ & $\begin{array}{r}74 \\
241 \\
315\end{array}$ & $\begin{array}{r}56 \\
363 \\
419\end{array}$ & $\begin{array}{l}228 \\
656 \\
884\end{array}$ & $\begin{array}{r}228 \\
1,028 \\
1,256\end{array}$ \\
\hline 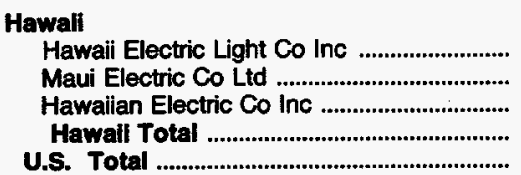 & $\begin{array}{l}\text { Investor-Owned } \\
\text { Investor-Owned } \\
\text { Investor-Owned }\end{array}$ & $\begin{array}{r}\overline{47} \\
713 \\
760 \\
2,348,094\end{array}$ & $\begin{array}{r}183 \\
131 \\
968 \\
1,282 \\
2,743,533\end{array}$ & $\begin{array}{r}229 \\
250 \\
392 \\
871 \\
3,002,972\end{array}$ & $\begin{array}{r}8,795 \\
4,100 \\
23,870 \\
36,765 \\
3,562,294\end{array}$ \\
\hline
\end{tabular}

Notes: -Data are final. •Data are provided for electric utilities with sales to ultimate consumers or sales for resale greater than or equal to 120,000 megawatthours.

Source: Energy Information Administration, Form ElA-861, "Annual Electric Utility Report:" 
Table 23. U.S. Electric Utility DSM Program Direct Utility Costs by North American Electric Reliability Council Region and Hawaii by DSM Program Category, 1993 (Thousand Dollars)

\begin{tabular}{|c|c|c|c|c|c|c|}
\hline $\begin{array}{c}\text { North American Electric Reliability } \\
\text { Council Region and Hawall / } \\
\text { Electric Utility }\end{array}$ & $\begin{array}{c}\text { Energy } \\
\text { Efficiency }\end{array}$ & $\begin{array}{l}\text { Direot Load } \\
\text { Control }\end{array}$ & Interruptible Load & $\begin{array}{l}\text { Other Load } \\
\text { Management }\end{array}$ & $\begin{array}{l}\text { Other Demand- } \\
\text { Side Management }\end{array}$ & $\begin{array}{l}\text { Total Direct } \\
\text { Utillty Cost }\end{array}$ \\
\hline \multicolumn{7}{|l|}{ ECAR } \\
\hline Appalachian Power Co ........................ & 1,383 & 0 & 0 & 90 & 0 & 1,473 \\
\hline Cincinnati Gas \& Electric Co ............ & 2,987 & 693 & 90 & 0 & 0 & 3,770 \\
\hline Cleveland Electric Illum Co ................ & 2,603 & 0 & 0 & 84 & 0 & 2,687 \\
\hline Columbus Southern Power Co ......... & 1,184 & 0 & 0 & 0 & 24 & 1,208 \\
\hline Consumers Power Co ........................ & 46,951 & 2,617 & 201 & 459 & 841 & 51,069 \\
\hline Crawfordsville Elec Lgt\&Pwr Co ...... & 9 & 0 & 0 & 0 & 0 & 9 \\
\hline Dayton Power \& Light Co ....................... & 23,941 & 0 & 0 & 0 & 0 & 23,941 \\
\hline Detroit Edison Co & 3,371 & 48 & 0 & 0 & 0 & 3,419 \\
\hline East Kentucky Power Coop Inc ........ & 1,000 & 0 & 0 & 300 & 0 & 1,300 \\
\hline Buckeye Power Inc ................................. & 0 & 0 & 2,015 & 0 & 0 & 2,015 \\
\hline 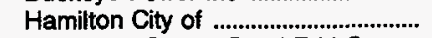 & 0 & 0 & 0 & 5 & 110 & 115 \\
\hline Hendricks County Rural E M C ....... & 0 & 60 & 0 & 0 & 0 & 60 \\
\hline Indianapolis Power \& Light Co ........ & 743 & 0 & 652 & 0 & 319 & 1,714 \\
\hline Indiana Michigan Power $\mathrm{Co}$ & 744 & 0 & 0 & 155 & 0 & 899 \\
\hline Kentucky Utilities Co ........................... & 612 & 0 & 0 & 49 & 0 & 661 \\
\hline Kingsport Power Co ............................. & 210 & 0 & 0 & 0 & 0 & 210 \\
\hline 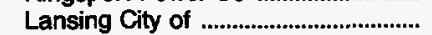 & 18 & 0 & 0 & 0 & 0 & 18 \\
\hline Louisville Gas \& Electric $\mathrm{Co}$ & 0 & 0 & 4,343 & 0 & 0 & 4,343 \\
\hline Midwest Electric Inc ............................ & 0 & 7 & 0 & 0 & 0 & 7 \\
\hline Monongahela Power Co .................... & 301 & 0 & 50 & 0 & 9 & 360 \\
\hline Ohio Edison $\mathrm{Co}$ & 2,669 & 0 & 12,119 & 63 & 0 & 14,851 \\
\hline Ohio Power Co & 638 & 0 & 0 & 0 & 625 & 1,263 \\
\hline 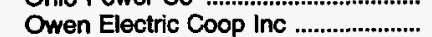 & 29 & 0 & 0 & 0 & 0 & $\begin{array}{r}1,200 \\
29\end{array}$ \\
\hline 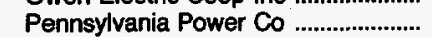 & 311 & 0 & 2,598 & 0 & 0 & 2,909 \\
\hline Potomac Edison Co & 1,133 & 0 & 0 & 0 & 16 & 1,149 \\
\hline 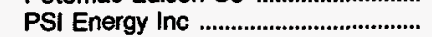 & 25,696 & 0 & 1,369 & 0 & 0 & 27,065 \\
\hline Southern Indiana Gas \& Elec Co .... & 1,695 & 1,499 & 1,304 & 0 & 0 & 4,498 \\
\hline South Central Power $\mathrm{Co} . . . . . . . . . . . . . . . . .$. & 90 & 475 & 0 & o & 110 & 675 \\
\hline 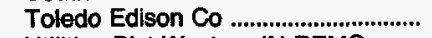 & 1,077 & 0 & 0 & 0 & 62 & 1,139 \\
\hline Utilities Dist-Western IN REMC ....... & 119 & 0 & 0 & 0 & 2 & 121 \\
\hline Virginia Tech Electric Service ........... & 0 & 0 & 0 & 6 & 0 & 6 \\
\hline West Penn Power Co & 1,689 & 0 & 0 & 0 & 498 & 2,187 \\
\hline Wheeling Power Co & 32 & 0 & 0 & 71 & 0 & 103 \\
\hline Wolverine Pwr Supply Coop Inc ...... & 0 & 885 & 0 & 0 & 0 & 885 \\
\hline Kentucky Power Co .............................. & 16 & 0 & 0 & 0 & 0 & 16 \\
\hline Wabash Valley Power Assn Inc ...... & 0 & 450 & 0 & 0 & 0 & 450 \\
\hline American Mun Power-Ohio Inc ........ & 25 & 0 & 0 & 0 & 35 & 60 \\
\hline 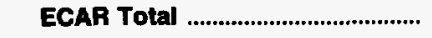 & 121,276 & 6,734 & 24,741 & 1,282 & 2,651 & 156,684 \\
\hline \multicolumn{7}{|l|}{ ERCOT } \\
\hline 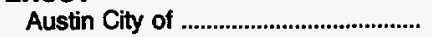 & 6,858 & 25 & 36 & 0 & 0 & 6,919 \\
\hline Brazos Electric Power Coop Inc ...... & 333 & 446 & 0 & 0 & 0 & 779 \\
\hline 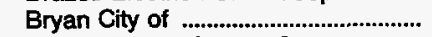 & 250 & 0 & 36 & 0 & 0 & 286 \\
\hline Central Power \& Light Co ................ & 2,021 & 0 & 0 & 3,352 & 0 & 5,373 \\
\hline 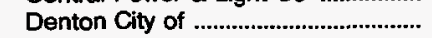 & 36 & 160 & 0 & 72 & 0 & 268 \\
\hline Garland City of & 0 & 329 & 297 & 0 & 0 & 626 \\
\hline Greenville Electric Util Sys ..................... & 0 & 12 & 0 & 0 & 0 & 12 \\
\hline Guadalupe Valley Elec Coop Inc .... & 0 & 78 & 0 & 0 & 0 & 78 \\
\hline Houston Lighting \& Power Co .......... & 9,021 & 5,082 & 0 & 3,483 & 0 & 17,586 \\
\hline Johnson County Elec Coop Assn ... & 111 & 0 & 0 & 0 & 0 & 111 \\
\hline Lower Colorado River Authority ........ & 2,506 & 293 & 0 & 0 & 0 & 2,799 \\
\hline Magic Valley Electric Coop Inc ........ & 88 & 0 & 0 & 0 & 0 & 88 \\
\hline Medina Electric Coop Inc .................. & 0 & 0 & 0 & 32 & 0 & 32 \\
\hline San Bernard Electric Coop Inc ........ & 13 & 0 & 0 & 43 & 0 & 56 \\
\hline Tri-County Electric Coop Inc ............ & 45 & 0 & 0 & 0 & 0 & 45 \\
\hline West Texas Utilities Co ....................... & 976 & 0 & 0 & 0 & 14 & 990 \\
\hline San Marcos City of ................................ & 58 & 19 & 0 & 0 & 0 & 77 \\
\hline Texas-New Mexico Power Co ............ & 480 & 0 & 0 & 0 & 180 & 660 \\
\hline Texas Utilities Electric Co ................... & 15,215 & 0 & 86 & 1,749 & 1,600 & 18,650 \\
\hline ERCOT Total & 38,011 & 6,444 & 455 & 8,731 & 1,794 & 55,435 \\
\hline \multicolumn{7}{|l|}{ MAAC } \\
\hline A \& N Electric Coop & & 145 & 0 & 0 & 0 & 145 \\
\hline Atlantic City Electric Co .......................... & 5,546 & 1,329 & 537 & 85 & 0 & 7,497 \\
\hline Baltimore Gas \& Electric Co ............ & 28,269 & 24,009 & 2,510 & 1,300 & 0 & 56,088 \\
\hline Choptank Electric Coop Inc ............... & 0 & 229 & 0 & 0 & 0 & 229 \\
\hline  & 313 & 15 & 0 & 0 & 101 & 429 \\
\hline Delmarva Power \& Light Co ............. & 1,728 & 5,944 & 0 & 23 & 0 & 7,695 \\
\hline Delaware Electric Coop Inc ................ & 0 & 662 & 0 & 0 & 0 & 662 \\
\hline
\end{tabular}

See footnotes at end of table. 
Table 23. U.S. Electric Utility DSM Program Direct Utility Costs by North American Electric Reliability Council Region and Hawail by DSM Program Category, 1993 (Thousand Dollars) (Continued)

\begin{tabular}{c|c|c|c|c|c|}
\hline $\begin{array}{c}\text { Morth American Electric Reliability } \\
\text { Council Region and Hawail / } \\
\text { Electric Utility }\end{array}$ & $\begin{array}{c}\text { Energy } \\
\text { Efficiency }\end{array}$ & $\begin{array}{c}\text { Direct Load } \\
\text { Control }\end{array}$ & Interruptible Load & $\begin{array}{c}\text { Other Load } \\
\text { Management }\end{array}$ & $\begin{array}{c}\text { Other Demand- } \\
\text { Side Management }\end{array}$ \\
\hline
\end{tabular}

MAC

Easton Utilities Comm

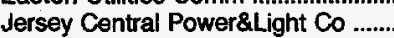

Metropolitan Edison Co

Pennsylvania Electric $\mathrm{Co}$

Philadelphia Electric Co

Potomac Electric Power Co ...............

Public Service Electric\&Gas Co ......

Southern Maryland El Coop Inc ......

UGI Utilities Inc

Somerset Rural Elec Coop Inc ..........

Adams Electric Coop Inc

Bedford Rural Elec CoOp Inc

Valley Rural Electric Coop Inc ..........

Central Electric Coop Inc

Claverack Rural Elec Coop Inc

Southwest Central R E C Corp .......

Northwestem Rural E C A Inc .........

MAAC Total

MAIN

Boone Electric Coop

Central Illinois Light Co

Coles-Moultrie Electric Coop ............

Columbia City of

Commonwealth Edison $\mathrm{Co}$

Cuivre River Electric Coop Inc .........

Eastern Illini Electric Coop.

Illinois Power $\mathrm{Co}$

Madison Gas \& Electric Co .............

Manitowoc Public Utilities

Marshfield City of ...

Menard Electric Coop

Shelby Electric CoO

Southeastern IL Elec Coop Inc .......

Southwestern Electric Coop Inc ......

Springfield City of

Tri-County Electric Coop Inc ..............

Union Electric Co

Wayne-White Counties Elec Coop

Wisconsin Electric Power Co

Wisconsin Power \& Light Co ............

Wisconsin Public Power Inc Sys ....

Wisconsin Public Service Corp

MAIN Total

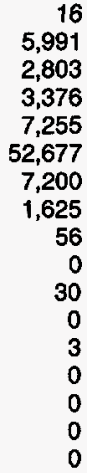

16

803

3,376

7,255

2,677

1,625

56
0

30

0

0

116,888

0
3,972

972
0

0

12,425

10,000

3,244

0

53

75

50

113

87

334

63,076

$0 \quad 68$

61
100

100
226

25

120
600

600

2

497

4,283

279

97

(a)

197

0

33,962

11,994

923

16,200

69,246

MAPP(U.S.)

Ames City of

Anoka City of

Austin City of

Electric Coop

Beatrice City of

Cedar Falls City of

Power Coop .................

Central Power Elec Coop Inc ..........

Clark Electric Coop

Coop Power Assn ...............................

Comhusker Public Power Dist

Dawson County Public Pwr Dist ......

East River Elec Power Coop inc .....

Fairmont Public Utilities Comm .........

Freebom-Mower Electric Coop .........

Grant-Lafayette Electric Coop .........

IES Utilities Inc

Interstate Power Co

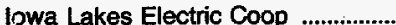

lowa-Illinois Gas\&Electric Co .............

L \& O Power Coop

See footnotes at end of table.

$\begin{array}{rr}2 & 98 \\ 0 & 76 \\ 45 & 37 \\ 31 & 6 \\ 0 & 127 \\ 209 & 0 \\ 612 & 0 \\ 0 & 84 \\ 0 & 12 \\ 1,289 & 4,597 \\ 0 & 24 \\ 0 & 0 \\ 0 & 2,479 \\ 0 & 200 \\ 0 & 43 \\ 5 & 30 \\ 2,657 & 245 \\ 1,939 & 2,379 \\ 142 & 1 \\ 1,502 & 0 \\ 0 & 20\end{array}$

$\begin{array}{rr}0 & 0 \\ 0 & 338 \\ 5 & 1,049 \\ 0 & 0 \\ 950 & 1,200 \\ 2,816 & 638 \\ 9,500 & 0 \\ 0 & 0 \\ 0 & 0 \\ 0 & 0 \\ 0 & 0 \\ 0 & 3 \\ 0 & 3 \\ 0 & 0 \\ 0 & 0 \\ 30 & 0 \\ 0 & 0 \\ 16,348 & 4,639\end{array}$

$\begin{array}{rr}0 & 16 \\ 1,956 & 12,257 \\ 0 & 3,857 \\ 0 & 3,376 \\ 1,200 & 10,606 \\ 0 & 68,556 \\ 20,000 & 46,700 \\ 0 & 4,869 \\ 0 & 56 \\ 0 & 53 \\ 100 & 519 \\ 0 & 78 \\ 0 & 56 \\ 0 & 113 \\ 0 & 87 \\ 0 & 30 \\ 0 & 334 \\ 23,357 & 224,308\end{array}$

0
0
0
0
2,400
0
0
0
0
0
0
0
0
0
0
0
0
0
0
0
800
0
0
100
3,300

$\begin{array}{rr}0 & 68 \\ 0 & 1,885 \\ 0 & 100 \\ 0 & 346 \\ 0 & 3,025 \\ 266 & 266 \\ 51 & 143 \\ 0 & 62 \\ 0 & 497 \\ 0 & 4,513 \\ 0 & 279 \\ 0 & 97 \\ 0 & 69 \\ 0 & 10 \\ 4 & 4 \\ 60 & 60 \\ 0 & 197 \\ 0 & 100 \\ 0 & 12,023 \\ 0 & 20 \\ 0 & 39,501 \\ 926 & 13,502 \\ 0 & 1,008 \\ 0 & 20,000 \\ 1,307 & 97,775\end{array}$

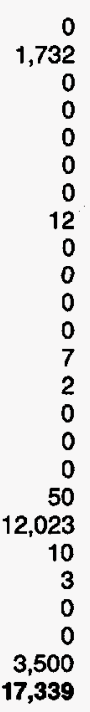

0
0
0
0
0
0
0
0
0
0
0
30
0
0
0
2
33
24
0
0
0
857

$3,37.6$
0,606

8,556

4,869

3

519

56

87

334

1,885

346

025

143

497

4,513

197

, 023

3,502

0,000

97,775

100
76
108
37
127
209
1,174
84
12
6,134
24
30
2,479
265
43
52
3,080
4,402
147
1,502
20

20 
Table 23. U.S. Electric Utility DSM Program Direct Utility Costs by North American Electric Reliability Council Region and Hawaii by DSM Program Category, 1993

(Thousand Dollars) (Continued)

\begin{tabular}{c|c|c|c|c|c|}
\hline $\begin{array}{c}\text { North American Electric Reliability } \\
\text { Council Region and Hawail / } \\
\text { Electric Utility }\end{array}$ & $\begin{array}{c}\text { Energy } \\
\text { Efficiency }\end{array}$ & $\begin{array}{c}\text { Direct Load } \\
\text { Control }\end{array}$ & Interruptible Load & $\begin{array}{c}\text { Other Load } \\
\text { Management }\end{array}$ & $\begin{array}{c}\text { Other Demand- } \\
\text { Side Management }\end{array}$ \\
\hline
\end{tabular}

\section{MAPP(U.S.)}

Lincoln Electric System .....................

Marshall City of

MDU Resources Group Inc ................

Nodak Electric Coop Inc

Midland Power Coop

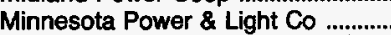

Minnkota Power Coop Inc

Moorhead City of

Muscatine City of

Nebraska Public Power District .........

Norris Public Power District

North Platte City of

Northern States Power

Northern States Power Co

Power Coop ............

Noblic Service Co

Oakdale Electric Coop

Oliver-Mercer Elec Coop Inc .............

Omaha Public Power District ...........

Otter Tail Power Co

Owatonna City of

People's Coop Power Assn .................

Pella City of

Pierre City



Rochester Public Utilities

Roseau Electric Coop Ino

Runestone Electric Assn

Shakopee Public Utilities Comm .....

Superior Water Light\&Power Co ......

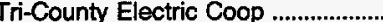

United Power Assn

Verendrye Electric Coop inc .............

Vernon Electric Coop

Mountrail-Williams EI CoO

Wild Rice Electric Coop Inc

Municipal Energy Agency of NE

Midwest Power Systems Inc

Cass County Electric Coop Inc ........

MAPP(U.S.) Total

NPCC(U.S.)

Bangor Hydro-Electric Co

Blackstone Valley Electric Co

Boston Edison Co

Braintree Town of

Burlington City of

Central Hudson Gas \& Elec Corp

Central Maine Power Co ...................

Central Vermont Pub Serv Corp ......

Chicopee City of ...

Commonwealth Electric Co ................

Concord Electric $\mathrm{Co}$.............................

Connecticut Light \& Power Co ........

Connecticut Valiey Elec Co Inc ......

Consolidated Edison Co-NY Inc .......

Eastern Edison Co

Exeter \& Hampton Electric Co

Fitchburg Gas \& Elec Light Co ........

Green Mountain Power Corp ...........

Holyoke City of

Hingham City of

Jamestown City of

Littleton Town of .

Maine Public Service

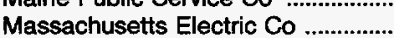

See footnotes at end of table.

\begin{tabular}{|c|c|}
\hline 68 & 0 \\
\hline 126 & 0 \\
\hline 0 & 257 \\
\hline 0 & 18 \\
\hline 79 & 1 \\
\hline 3,437 & 0 \\
\hline 0 & 1,973 \\
\hline 0 & 0 \\
\hline 247 & 0 \\
\hline 0 & 27 \\
\hline 0 & 58 \\
\hline 0 & 82 \\
\hline 3,607 & 253 \\
\hline 24,973 & 6,230 \\
\hline 48 & 313 \\
\hline 0 & 0 \\
\hline 0 & 67 \\
\hline 0 & 15 \\
\hline 459 & 0 \\
\hline 1,420 & 0 \\
\hline 0 & 57 \\
\hline 0 & 8 \\
\hline 126 & 25 \\
\hline 15 & 1 \\
\hline 336 & 0 \\
\hline 114 & 292 \\
\hline 0 & 65 \\
\hline 16 & 80 \\
\hline 0 & 0 \\
\hline 188 & 11 \\
\hline 1 & 132 \\
\hline 679 & 1,500 \\
\hline 0 & 25 \\
\hline 14 & 53 \\
\hline 16 & 57 \\
\hline 0 & 45 \\
\hline 3 & 20 \\
\hline 6,274 & 2,999 \\
\hline 12 & \\
\hline 50,691 & 25,187 \\
\hline
\end{tabular}

$\begin{array}{rr}0 & 9 \\ 1 & 0 \\ 0 & 0 \\ 0 & 0 \\ 0 & 0 \\ 0 & 0 \\ 0 & 0 \\ 35 & 0 \\ 0 & 0 \\ 0 & 0 \\ 0 & 0 \\ 0 & 0 \\ 55 & 1 \\ 2,139 & 748 \\ 0 & 949 \\ 6 & 0 \\ 0 & 0 \\ 0 & 0 \\ 0 & 0 \\ 112 & 0\end{array}$

9
0
0
0
0
0
0
0
0
0
0
1
748
949
0
0
0
0
0

1,11

1,112
5

$\begin{array}{ll}0 & 0 \\ 0 & 0 \\ 0 & 0\end{array}$

0
0

0

0
0

0

0

0
0

5,248

0
8,752

0
0
$\mathbf{3 , 6 3 0}$

$\begin{array}{rr}113 & \\ 0 & \\ 0 & 35 \\ 35 & \\ 0 & \\ 0 & \\ 0 & \end{array}$

0
0
355

2,333

44,070

65
954

2,391

3,688

12,700

5,908

110

3,498

366
573

7,543

273
100,162

5,868

372

660

4,289

25

25

120
4

24,932

77

38,535

\begin{tabular}{|c|c|}
\hline 0 & 77 \\
\hline 76 & 203 \\
\hline 0 & 257 \\
\hline 0 & 18 \\
\hline 0 & 80 \\
\hline 0 & 3,437 \\
\hline 0 & 1,973 \\
\hline 0 & 35 \\
\hline 0 & 247 \\
\hline 0 & 27 \\
\hline 0 & 58 \\
\hline 0 & 83 \\
\hline 0 & 4,663 \\
\hline 0 & 34,291 \\
\hline 0 & 361 \\
\hline 0 & 6 \\
\hline 0 & 67 \\
\hline 0 & 15 \\
\hline 0 & 459 \\
\hline 20 & 3,664 \\
\hline 0 & 62 \\
\hline 0 & 8 \\
\hline 0 & 151 \\
\hline 0 & 16 \\
\hline 0 & 336 \\
\hline 0 & 406 \\
\hline 0 & 65 \\
\hline 0 & 102 \\
\hline 0 & 30 \\
\hline 0 & 202 \\
\hline 0 & 133 \\
\hline 2,500 & 4,679 \\
\hline 0 & 50 \\
\hline 0 & 70 \\
\hline 0 & 73 \\
\hline 0 & 45 \\
\hline 0 & 23 \\
\hline 254 & 14,775 \\
\hline 0 & 77 \\
\hline 3,169 & 91,429 \\
\hline
\end{tabular}

1,115

2,333

44,425

101

954

2,395
4,236

13,850

6,758

130

3,534

366

37,573

343

110,485

5,868

372

660

25

25

120

27,642

104

40,599 
Table 23. U.S. Electric Utility DSM Program Direct Utility Costs by North American Electric Reliability Council Region and Hawaii by DSM Program Category, 1993

(Thousand Dollars) (Continued)

\begin{tabular}{|c|c|c|c|c|c|c|}
\hline $\begin{array}{l}\text { North American Electric Reliabillty } \\
\text { Councll Reglon and Hawali / } \\
\text { Electric Utility }\end{array}$ & $\begin{array}{l}\text { Energy } \\
\text { Efficiency }\end{array}$ & $\begin{array}{l}\text { Direct Load } \\
\text { Control }\end{array}$ & Interruptible Load & $\begin{array}{l}\text { Other Load } \\
\text { Management }\end{array}$ & $\begin{array}{c}\text { Other Demand- } \\
\text { Side Management }\end{array}$ & $\begin{array}{l}\text { Total Direct } \\
\text { Utility Cost }\end{array}$ \\
\hline
\end{tabular}

NPCC(U.S.)

Massena Town of

Montaup Electric Co

Narragansett Electric Co

New England Power Co

New Hampshire Elec Coop inc ........

New York State Elec \& Gas Corp ..

Newport Electric Corp

Niagara Mohawk Power Corp ............

Norwood City of

Orange \& Rockland Utils Inc

Power Authority of State of NY .......

Public Service Co of $\mathrm{NH}$

Reading Town of

Shrewsbury Town of

United llluminating Co

Omya Inc

Western Massachusetts Elec Co ....

Granite State Electric Co ..

NPCC(U.S.) Total

SERC

Aiken Electric Coop Inc .

Alabama Electric Coop Inc ...............

Alabama Power Co

Albemarle City of

Altamaha Electric Member Corp

Amicalola Electric Member Corp .....

BARC Electric Coop Inc

Berkeley Electric Coop Inc ...

Black River Electric Coop Inc

Blue Ridge Elec Member Corp ......

Canoochee Electric Member Corp.

Carolina Power \& Light Co .

Carroll Electric Member Corp ...........

Carteret-Craven El Member Corp ....

Central Florida Elec Coop Inc ..........

Central Georgia EI Member Corp ....

Central Electric Member Corp ..........

Clay Electric Coop Inc

Coast Electric Power Assn ................

Coastal Electric Member Corp .........

Cobb Electric Membership Corp ....

Community Electric Coop

Coweta-Fayette El Member Corp ....

Crescent Electric Member Corp ......

Davidson Electric Member Corp .....

Douglas City of

Duke Power Co .....

Elizabeth City

Excelsior Electric Member Corp ......

Fairtield Electric Coop Inc

Fayetteville Public Works Comm ....

Fitzgerald Wtr Lgt \& Bond Comm

Flint Electric Membership Corp .......

Florida Keys El Coop Assn Inc ........

Florida Power \& Light Co ..................

Florida Power Corp

Fort Pierce Utilities Auth .......................

Four County Elec Member Corp .....

Gaffney City of ....................................

Gainesville Regional Utilities .............

GreyStone Power Corp .

Georgia Power Co ...............................

Grady County Elec Member Corp ...

Greenville Utilities Comm

Griffin City of

10
9,276
9,991
0
366
43,653
1,175
24,944
190
16,078
9,485
371
10
9,109
35
378
12,414
108
14,923
1,746
444,232

3
0
644
0
367
0
0
0
2
57
0
0
15
0
228
0
0
0
20
90
5,994

0
0
0
2,797
0
0
0
0
29
1,856
0
0
50
0
0
0
200
0
0
0
12,339

0
0
0
10
367
0
0
0
11
0
0
0
0
0
0
0
350
0
0
0
871

0
0
0
0
0
0
0
0
0
1,467
0
0
80
120
0
0
0
0
0
0
8,219

13
9,276

10,635

2,807

1,100

43,653

1,175

24,944

232

19,458

9,485

371

155

9,229

263
378

12,964

108

14,943

1,836

471,655

390

390
4,473

4,473

12

0
38

38
0

15,400

0

268
16
68

2,714

53

1,380

137

111

20
3

3
6,102

288

288
0
90
0
7
1,000
178
78,702
70,807
0
550
1,802
0
353
214
6
206
25

484

19,671

160

50

98

450

408

5

38,700

68

268
16

73

79

2,730
50

133

1,560

137

325

782

30
6

54,097

288

45
146

146
25
7

7
, 258

178

128,246

98,146

163

1,083

1,802

154

2,294

17
266

25

See footnotes at end of table. 
Table 23. U.S. Electric Utility DSM Program Direct Utility Costs by North American Electric Reliability Council Region and Hawaii by DSM Program Category, 1993

(Thousand Dollars) (Continued)

\begin{tabular}{c|c|c|c|c|c|}
\hline $\begin{array}{c}\text { North American Electric Reliabillty } \\
\text { Council Region and Hawail / } \\
\text { Electric Utility }\end{array}$ & $\begin{array}{c}\text { Energy } \\
\text { Efficlency }\end{array}$ & $\begin{array}{c}\text { Direct Load } \\
\text { Control }\end{array}$ & Interruptible Load & $\begin{array}{c}\text { Other Load } \\
\text { Management }\end{array}$ & $\begin{array}{c}\text { Other Demand- } \\
\text { Side Management }\end{array}$ \\
\hline
\end{tabular}

SERC

Gulf Power Co

Harrisonburg City of

Hart Electric Member Corp ..............

Haywood Electric Member Corp ......

High Point Town of

Jackson Electric Member Corp

Jacksonville Electric Auth

Jefferson Electric Member Corp ......

Jones-Onslow Elec Member Corp .

Kinston City of

Kissimmee Utility Authority ................

Lakeland City of

Lamar Electric Membership Corp ...

Laurens Electric Coop Inc .................

Laurinburg City of

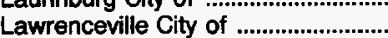

Lee County Electric Coop Inc .........

Leesburg City of .................................

Lumbee River Elec Member Corp ..

Lumberton City of

Lynches River Elec Coop Inc

Manassas City of ..................................

Marietta City of

Mecklenburg Electric Coop Inc ........

Mid-Carolina Electric Coop Inc .......

Mississippi Power $\mathrm{Co}$...

Mitchell Electric Member Corp ........

Monroe City of

Municipal Electric Authority ................

New Bern City of

New River Light \& Power Co .............

New Smyrna Beach Utils Comm .....

North Carolina Mun Power Agny .....

Northern Virginia Elec Coop ...

North Carolina El Member Corp .....

North Carolina Eastern M P A .........

Northern Neck Elec Coop Inc .........

Ocala City of .

Palmetto Electric Coop Inc

Orlando Utilities Comm

Planters Electric Member Corp .........

Pee Dee Electric Member Corp .......

Piedmont Electric Member Corp ....

Prince George Electric Coop

Randolph Electric Member Corp .....

Rayle Electric Membership Corp ....

Reedy Creek Improvement Dist ......

Roanoke Electric Member Corp ......

Rock Hill City of .

Rocky Mount City of ...........................

Satilla Rural Elec Member Corp .....

Savannah Electric \& Power Co .......

Sawnee Electric Members Corp ......

Shenandoah Valley Elec Coop ........

Singing River Elec Power Assn .......

South Carolina Electric\&Gas Co ....

South Carolina Pub Serv Auth .........

South Mississippi El Pwr Assn .........

Snapping Shoals El Member Corp .

Sumter Electric Coop Inc

Suwannee Valley Elec Coop Inc ...

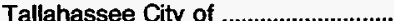

Tampa Electric Co

Tennessee Valley Authority ...............

Thomasville City of

1,958

32

150

2

129

845

11

0

114

0
0
27

27

0
0
323

323

0

$\begin{array}{ll}0 \\ 0 & 230\end{array}$

(0)

0
1

106

(0)

0

$\begin{array}{ll}0 & 210 \\ 0 & 24\end{array}$

$\begin{array}{rr}0 & 62 \\ 0 & 1,131\end{array}$

$170 \quad 851$

851
4,375

1,325

10

65

65
0

0

194

20

0
7
0

0
23

23
737

737
125

25

0
426

69

0
27

27
5,263

7,477

2,787

84

329

0

330

4,015

5,475

Tri-County Elec Member Corp

Tideland Electric Member Corp ........

Troup Electric Members Corp

See footnotes at end of table. 
Table 23. U.S. Electric Utility DSM Program Direct Utility Costs by North American Electric Reliability Council Region and Hawail by DSM Program Category, 1993 (Thousand Dollars) (Continued)

\begin{tabular}{|c|c|c|c|c|c|c|}
\hline $\begin{array}{c}\text { North American Electric Reliability } \\
\text { Councll Region and Hawail / } \\
\text { Electric Utilty }\end{array}$ & $\begin{array}{l}\text { Energy } \\
\text { Efficiency }\end{array}$ & $\begin{array}{l}\text { Direct Load } \\
\text { Control }\end{array}$ & Interruptible Load & $\begin{array}{l}\text { Other Load } \\
\text { Management }\end{array}$ & $\begin{array}{c}\text { Other Demand- } \\
\text { Side Management }\end{array}$ & $\begin{array}{l}\text { Total Direct } \\
\text { Utility Cost }\end{array}$ \\
\hline \multicolumn{7}{|l|}{ SERC } \\
\hline Union Electric Membership Corp ..... & 0 & 12 & 3 & 0 & 0 & 15 \\
\hline Virginia Electric \& Power Co .............. & 8,654 & 11,955 & 6,813 & 390 & 0 & 27,812 \\
\hline Wake Electric Membership Corp ..... & 235 & 182 & 0 & 0 & 0 & 417 \\
\hline Walton Electric Member Corp ........... & 101 & 288 & 0 & 0 & 52 & 441 \\
\hline Washington City of & 0 & 147 & 100 & 0 & 0 & 247 \\
\hline Washington Elec Member Corp ........ & 0 & 5 & 0 & 0 & 0 & 5 \\
\hline Withlacoochee River Elec Coop ....... & 53 & 0 & 0 & 0 & 6 & 58 \\
\hline York Electric Coop Inc ....................... & 0 & 0 & 0 & 3 & 0 & 3 \\
\hline Southside Electric Coop Inc .............. & 0 & 46 & 2 & 2 & 0 & 50 \\
\hline Brunswick Electric Member Corp .... & 150 & 417 & 13 & 0 & 0 & 580 \\
\hline Colquitt Electric Members Corp ........ & 0 & 0 & 0 & 255 & 0 & 255 \\
\hline Central Electric Pwr Coop Inc .......... & 0 & 1,519 & 0 & 0 & 0 & 1,519 \\
\hline Rappahannock Electric Coop ........... & 0 & 553 & 0 & 0 & 0 & 553 \\
\hline 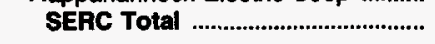 & 167,308 & 236,335 & 95,948 & 2,790 & 3,000 & 505,381 \\
\hline \multicolumn{7}{|l|}{ SPP } \\
\hline Arkansas Power \& Light Co .............. & 0 & 123 & 0 & 0 & 0 & 123 \\
\hline Bailey County Elec Coop Assn ......... & 0 & 0 & 6 & 0 & 0 & 6 \\
\hline 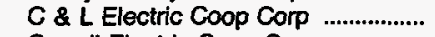 & 0 & 0 & 5 & 0 & 0 & 5 \\
\hline Carroll Electric Coop Corp ................ & 0 & 85 & 0 & 0 & 0 & 85 \\
\hline Central Rural Electric Coop .............. & 0 & 73 & 0 & 0 & 0 & 73 \\
\hline Caddo Electric Coop Inc .................... & 0 & 69 & 0 & 0 & 0 & 69 \\
\hline Cookson Hills Elec Coop Inc ............ & 0 & 390 & 0 & 0 & 0 & 390 \\
\hline Craighead Electric Coop Corp .......... & 0 & 0 & 326 & 0 & 0 & 326 \\
\hline 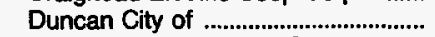 & 15 & 0 & 0 & 0 & 0 & 15 \\
\hline Empire District Electric Co ................. & 0 & 0 & 495 & 0 & 0 & 495 \\
\hline First Electric Coop Corp ..................... & 0 & 967 & 0 & 0 & 0 & 967 \\
\hline Gulf States Utilities Co ........................ & 2,500 & 0 & 0 & 0 & 0 & 2,500 \\
\hline Independence City of ........................... & 105 & 0 & 0 & 0 & 0 & 105 \\
\hline Indian Electric CoOp Inc ..................... & 0 & 48 & 0 & 0 & 0 & 48 \\
\hline Kansas Electric Power Coop Inc ..... & 0 & 73 & 0 & 0 & 0 & 73 \\
\hline Kansas City Power \& Light Co ......... & 0 & 390 & 670 & 0 & 0 & 1,060 \\
\hline Kansas Gas \& Electric Co ............... & 0 & 971 & 0 & 554 & 0 & 1,525 \\
\hline Mississippi Cnty Elec Coop Inc ........ & 0 & 36 & 0 & 0 & 0 & 36 \\
\hline North Arkansas Elec Coop Inc ......... & 0 & 228 & 0 & 0 & 0 & 228 \\
\hline Northeast Louisiana Power Coop .... & 0 & 0 & 0 & 59 & 0 & 59 \\
\hline Oklahoma Gas \& Electric Co ............. & 1,064 & 5,718 & 11,083 & 882 & 0 & 18,747 \\
\hline Osceola City of & 0 & 300 & 0 & 0 & 0 & 300 \\
\hline Ozark Electric Coop Inc .......................... & 1 & 0 & 1 & 0 & 0 & 2 \\
\hline Petit Jean Electric Coop Corp .......... & 0 & 109 & 43 & 0 & 0 & 152 \\
\hline Red River Valley Rrl Elec Assn ....... & 82 & 0 & 0 & 0 & 2 & 84 \\
\hline South Central Ark El Coop Inc ......... & 0 & 2 & 0 & 0 & $\overline{0}$ & 2 \\
\hline South Plains Electric Coop Inc ......... & 182 & 270 & 0 & 0 & 0 & 452 \\
\hline Southwestern Electric Power Co ..... & 527 & 0 & 0 & 0 & 0 & 527 \\
\hline Southwestern Public Service Co ..... & 935 & 0 & 39 & 0 & 0 & 974 \\
\hline Verdigris Valley Elec Coop Inc ......... & 0 & 95 & 4 & 0 & 0 & 99 \\
\hline White River Valley El Coop inc ........ & 0 & 0 & 2 & 0 & 0 & 2 \\
\hline Woodruff Electric Coop Corp ............. & 0 & 52 & 0 & 5 & 0 & 57 \\
\hline Western Resources Inc .................... & 0 & 691 & 1,632 & 0 & 295 & 2,618 \\
\hline 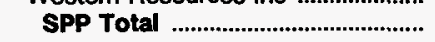 & 5,411 & 10,690 & 14,306 & 1,500 & 297 & 32,204 \\
\hline \multicolumn{7}{|l|}{ WSCC(U.S.) } \\
\hline Alameda City of & 223 & 0 & 24 & 0 & 0 & 247 \\
\hline  & 506 & 349 & 65 & 0 & 0 & 920 \\
\hline Arizona Electric Pwr Coop Inc .......... & 400 & 0 & 0 & 0 & 0 & 400 \\
\hline Arizona Public Service Co .................. & 3,720 & 0 & 0 & 0 & 0 & 3,720 \\
\hline Ashland City of & 100 & 0 & 0 & 0 & 0 & 100 \\
\hline Bonneville Power Admin ..................... & 102,400 & 0 & 13,369 & 0 & 9,600 & 125,369 \\
\hline Bountiful City City of ............................. & 20 & 0 & 40 & 0 & 0 & 60 \\
\hline Colton City of & 0 & 154 & 0 & 0 & 0 & 154 \\
\hline  & 747 & 0 & 0 & 0 & 24 & 771 \\
\hline 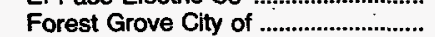 & 213 & 0 & 0 & 0 & 0 & 213 \\
\hline 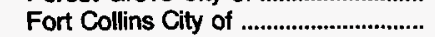 & 0 & 110 & 0 & 22 & 0 & 132 \\
\hline 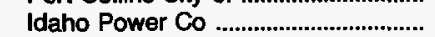 & 8,575 & 0 & 0 & 0 & 0 & 8,575 \\
\hline Imperial Irrigation District .................... & 359 & 0 & 0 & 0 & 134 & 493 \\
\hline La Plata Electric Assn Inc ................ & 0 & 0 & 0 & 0 & 10 & 10 \\
\hline 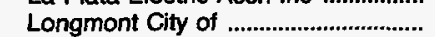 & 63 & 0 & 0 & 0 & 0 & 63 \\
\hline 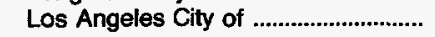 & 15,348 & 0 & 278 & 442 & 0 & 16,068 \\
\hline
\end{tabular}

See tootnotes at end of table. 
Table 23. U.S. Electric Utility DSM Program Direct Utility Costs by North American Electric Reliability Council Region and Hawail by DSM Program Category, 1993

(Thousand Dollars) (Continued)

\begin{tabular}{|c|c|c|c|c|c|c|}
\hline $\begin{array}{c}\text { North American Electric Reliability } \\
\text { Council Region and Hawall / } \\
\text { Electric Utility }\end{array}$ & $\begin{array}{c}\text { Energy } \\
\text { Efficlency }\end{array}$ & $\begin{array}{l}\text { Direct Load } \\
\text { Control }\end{array}$ & Interruptible Load & $\begin{array}{l}\text { Other Load } \\
\text { Management }\end{array}$ & $\begin{array}{c}\text { Other Demand- } \\
\text { Side Management }\end{array}$ & $\begin{array}{l}\text { Total Direct } \\
\text { Utility Cost } 1\end{array}$ \\
\hline  & $\begin{array}{r}70 \\
776 \\
7,049 \\
3 \\
2,421 \\
5 \\
105,863 \\
41,000 \\
670 \\
259 \\
16,857 \\
7,088 \\
0 \\
57,173 \\
22 \\
203 \\
175 \\
33,920 \\
1,730 \\
26,249 \\
0 \\
19,570 \\
3,278 \\
100,117 \\
1,336 \\
80 \\
8,430 \\
0 \\
805 \\
42 \\
0 \\
0 \\
22,805 \\
0 \\
2 \\
2,840 \\
593,512 \\
606,575\end{array}$ & $\begin{array}{r}0 \\
378 \\
0 \\
26 \\
3,951 \\
0 \\
0 \\
0 \\
0 \\
192 \\
0 \\
0 \\
0 \\
0 \\
9 \\
0 \\
83 \\
0 \\
92 \\
162 \\
0 \\
0 \\
0 \\
1,181 \\
0 \\
0 \\
0 \\
0 \\
0 \\
0 \\
0 \\
367,730\end{array}$ & $\begin{array}{r}0 \\
0 \\
0 \\
0 \\
108 \\
0 \\
1,417 \\
0 \\
230 \\
0 \\
0 \\
0 \\
0 \\
0 \\
35 \\
0 \\
0 \\
0 \\
0 \\
248 \\
400 \\
0 \\
0 \\
2,014 \\
0 \\
0 \\
0 \\
10 \\
0 \\
0 \\
18,238 \\
208,466\end{array}$ & $\begin{array}{r}0 \\
0 \\
0 \\
50 \\
62 \\
0 \\
17,283 \\
0 \\
0 \\
56 \\
0 \\
600 \\
0 \\
0 \\
58 \\
537 \\
0 \\
0 \\
786 \\
328 \\
0 \\
0 \\
0 \\
3,412 \\
0 \\
0 \\
0 \\
0 \\
0 \\
32 \\
50,595\end{array}$ & $\begin{array}{r}16 \\
0 \\
0 \\
22 \\
0 \\
0 \\
0 \\
0 \\
0 \\
0 \\
0 \\
0 \\
156 \\
0 \\
0 \\
0 \\
35 \\
0 \\
499 \\
173 \\
0 \\
0 \\
0 \\
0 \\
0 \\
0 \\
0 \\
0 \\
0 \\
54,479\end{array}$ & $\begin{array}{r}86 \\
1,154 \\
7,049 \\
101 \\
6,542 \\
5 \\
124,563 \\
41,000 \\
900 \\
507 \\
16,857 \\
7,688 \\
156 \\
57,173 \\
124 \\
740 \\
293 \\
33,920 \\
3,107 \\
27,160 \\
400 \\
19,570 \\
3,278 \\
106,724 \\
1,336 \\
80 \\
8,430 \\
10 \\
805 \\
74 \\
2 \\
33 \\
22,805 \\
164 \\
3 \\
2,840 \\
652,974 \\
2,287,845\end{array}$ \\
\hline 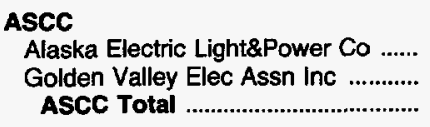 & $\begin{array}{r}0 \\
125 \\
125\end{array}$ & $\begin{array}{r}45 \\
0 \\
45\end{array}$ & $\begin{array}{l}0 \\
0 \\
0\end{array}$ & $\begin{array}{l}0 \\
0 \\
0\end{array}$ & $\begin{array}{l}0 \\
0 \\
0\end{array}$ & $\begin{array}{r}45 \\
125 \\
170\end{array}$ \\
\hline 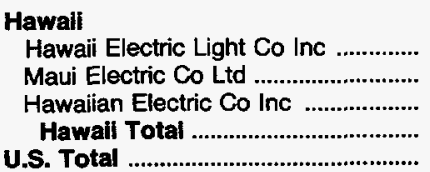 & $\begin{array}{r}183 \\
101 \\
968 \\
1,252 \\
1,607,952\end{array}$ & $\begin{array}{r}0 \\
0 \\
0 \\
0 \\
367,775\end{array}$ & $\begin{array}{r}0 \\
0 \\
0 \\
0 \\
208,466\end{array}$ & $\begin{array}{r}0 \\
0 \\
0 \\
0 \\
50,595\end{array}$ & $\begin{array}{r}0 \\
0 \\
0 \\
0 \\
54,479\end{array}$ & $\begin{array}{r}183 \\
101 \\
968 \\
1,252 \\
2,289,267\end{array}$ \\
\hline
\end{tabular}

1 Reflects electric utility cost incurred during the year that are identified with one of the demand-side management program categories.

Notes: -Data are final. -Data are provided for electric utilities with sales to ultimate consumers or sales for resale greater than or equal to 120,000 megawatthours.

Source: Energy Information Administration, Form EIA-861, "Annual Electric Utility Report." 
Table 24. U.S. Electric Utility DSM Program Indirect Utility Costs by North American Electric Reliability Council Region and Hawail by Cost Category, 1993 (Thousand Dollars)

\begin{tabular}{|c|c|c|c|c|c|}
\hline $\begin{array}{l}\text { North American Electric Rellability } \\
\text { Council Region and Hawall / } \\
\text { Electric Utility }\end{array}$ & Administrative & Marketing & $\begin{array}{l}\text { Monitoring } \\
\text { and } \\
\text { Evaluation }\end{array}$ & Other ${ }^{1}$ & $\begin{array}{l}\text { Total Indirect } \\
\text { Utlity Cost }\end{array}$ \\
\hline \multicolumn{6}{|l|}{ ECAR } \\
\hline 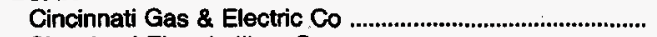 & 0 & 0 & 0 & 418 & 418 \\
\hline 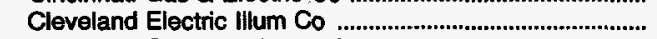 & 475 & 254 & 0 & 0 & 729 \\
\hline Columbus Southern Power $\mathrm{Co}$ & 41 & 0 & 8 & 1,812 & 1,861 \\
\hline Consumers Power $\mathrm{CO}$ & 0 & 0 & 1,497 & 0 & 1,497 \\
\hline 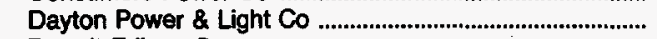 & 0 & 0 & 1,412 & 0 & 1,412 \\
\hline Detroit Edison Co & 188 & 0 & 214 & 275 & 677 \\
\hline 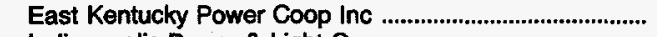 & 400 & 100 & 100 & 0 & 600 \\
\hline Indianapolis Power \& Light Co & 0 & 0 & 0 & 843 & 843 \\
\hline Kentucky Utilities Co & 621 & 1,060 & 28 & 0 & 1,709 \\
\hline 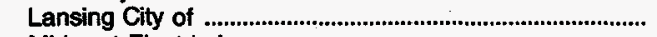 & 0 & 8 & 0 & 0 & 8 \\
\hline Midwest Electric Inc & 0 & 154 & 0 & 0 & 154 \\
\hline 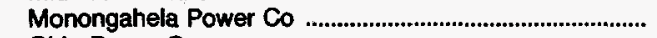 & 0 & 97 & 0 & 0 & 97 \\
\hline Ohio Power Co & 0 & 0 & 10 & 1,423 & 1,433 \\
\hline 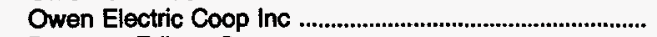 & 0 & 67 & 0 & 0 & 67 \\
\hline Potomac Edison Co & 339 & 30 & 2 & 0 & 371 \\
\hline 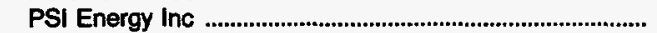 & $17 t$ & 6,976 & 1,187 & 0 & 8,334 \\
\hline 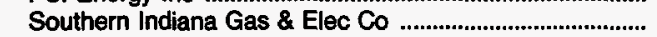 & 473 & 582 & 270 & 46 & 1,371 \\
\hline 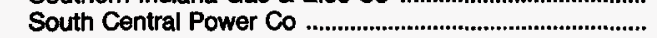 & 15 & 10 & 0 & 0 & 25 \\
\hline 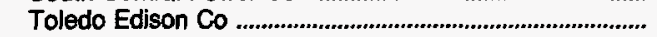 & 255 & 162 & 0 & 0 & 417 \\
\hline 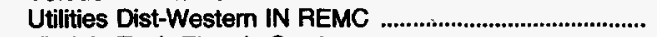 & 80 & 55 & 6 & 0 & 141 \\
\hline 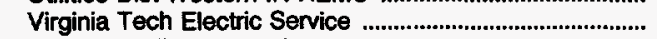 & 2 & 0 & 2 & 0 & 4 \\
\hline Wabash Valley Power Assn Inc ........................................... & 100 & 100 & 100 & 7,945 & 8,245 \\
\hline 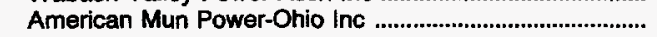 & 21 & 0 & 5 & 14 & 40 \\
\hline ECAR Total & $\mathbf{3 , 1 8 1}$ & 9,655 & 4,841 & 12,776 & 30,453 \\
\hline \multicolumn{6}{|l|}{ ERCOT } \\
\hline Austin City of & 2,413 & 347 & 160 & 0 & 2,920 \\
\hline Bryan City of & 60 & 20 & 0 & 0 & 80 \\
\hline 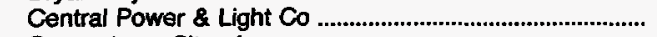 & 503 & 452 & 268 & 0 & 1,223 \\
\hline Georgetown City of & 36 & 0 & 0 & 0 & 36 \\
\hline 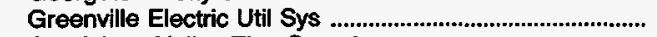 & 5 & 1 & 1 & 0 & 7 \\
\hline 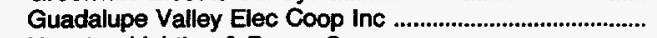 & 26 & 26 & 28 & 0 & 80 \\
\hline 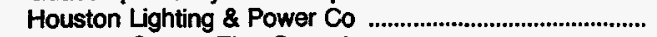 & 0 & 0 & 336 & 0 & 336 \\
\hline Johnson County Elec Coop Assn ...................................... & 5 & 3 & 0 & 0 & 8 \\
\hline Lower Colorado River Authority .......................................... & 155 & 94 & 51 & 212 & 512 \\
\hline 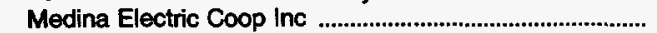 & 18 & 0 & 24 & 12 & 54 \\
\hline 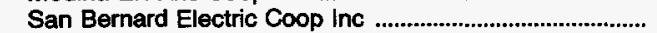 & 0 & 0 & 0 & 3 & 3 \\
\hline 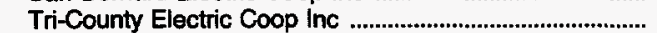 & 0 & 7 & 0 & 0 & 7 \\
\hline 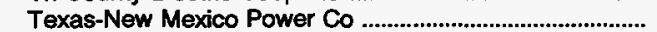 & 0 & 0 & 0 & 380 & 380 \\
\hline Texas Utilities Electric $C_{0}$ & 1,212 & 0 & 240 & 0 & 1,452 \\
\hline ERCOT Total & 4,433 & 950 & 1,108 & 607 & 7,098 \\
\hline \multicolumn{6}{|l|}{ MAAC } \\
\hline Atlantic City Electric Co & 2,219 & 285 & 329 & 67 & 2,900 \\
\hline 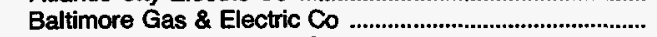 & 4,700 & 3,138 & 1,757 & 0 & 9,595 \\
\hline 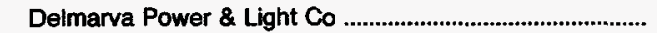 & 717 & 0 & 451 & 39 & 1,207 \\
\hline Easton Utilities Comm & 25 & 5 & 3 & 0 & 33 \\
\hline 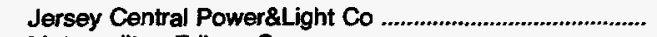 & 0 & 0 & 0 & 1,428 & 1,428 \\
\hline 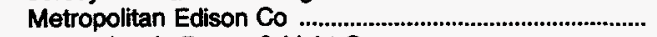 & 238 & 0 & 0 & 366 & 604 \\
\hline Pennsylvania Power \& Light Co .......................................... & 4,525 & 3,520 & 0 & 5,005 & 13,050 \\
\hline 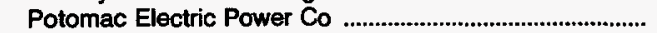 & 3,272 & 1,065 & 623 & 0 & 4,960 \\
\hline 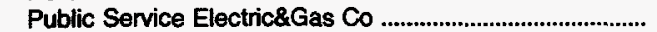 & 1,050 & 1,200 & 1,000 & 250 & 3,500 \\
\hline 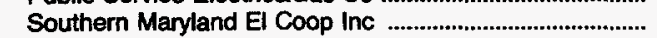 & 102 & 0 & 202 & 0 & 304 \\
\hline 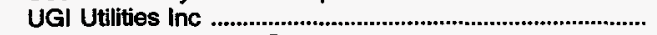 & 13 & 6 & 15 & 7 & 41 \\
\hline Somerset Rural Elec Coop Inc & 44 & 23 & 3 & 0 & 70 \\
\hline 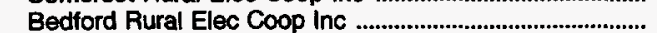 & 0 & 15 & 13 & 0 & 28 \\
\hline 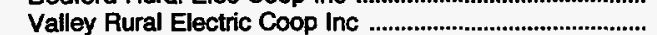 & 25 & 25 & 0 & 0 & 50 \\
\hline 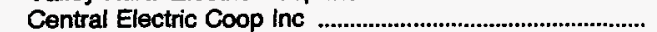 & 3 & 16 & 0 & 0 & 19 \\
\hline Claverack Rural Elec Coop Inc & 14 & 0 & 0 & 0 & 14 \\
\hline 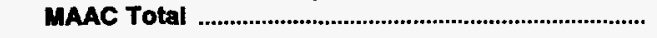 & 16,947 & 9,298 & 4,396 & 7,162 & 37,803 \\
\hline \multicolumn{6}{|l|}{ MAIN } \\
\hline 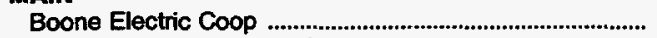 & 2 & 2 & 0 & 0 & 4 \\
\hline Central lllinois Pub Serv Co & 0 & 0 & 0 & 691 & 691 \\
\hline 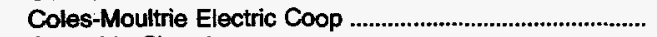 & 0 & 50 & 0 & 0 & 50 \\
\hline Columbia City of & 120 & 80 & 43 & 0 & 243 \\
\hline 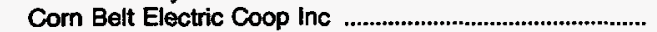 & 7 & 10 & 0 & 0 & 17 \\
\hline 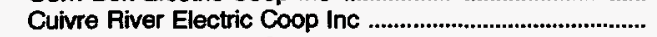 & 0 & 2 & 2 & 0 & 4 \\
\hline 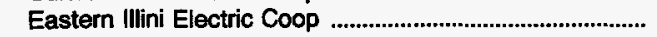 & 10 & 20 & 10 & 0 & 40 \\
\hline
\end{tabular}

See footnotes at end of table. 
Table 24. U.S. Electric Utility DSM Program Indirect Utility Costs by North American Electric Reliability Council Region and Hawail by Cost Category, 1993

(Thousand Dollars) (Continued)

\begin{tabular}{|c|c|c|c|c|c|}
\hline $\begin{array}{c}\text { North American Electric Reliability } \\
\text { Council Region and Hawail / } \\
\text { Electric Utility }\end{array}$ & Administrative & Marketing & $\begin{array}{l}\text { Monitoring } \\
\text { and } \\
\text { Evaluation }\end{array}$ & Other ${ }^{1}$ & $\begin{array}{l}\text { Total Indirec } \\
\text { Utility Cost }\end{array}$ \\
\hline \multicolumn{6}{|l|}{ MAIN } \\
\hline 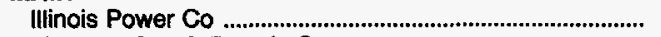 & 0 & 0 & 0 & 98 & 98 \\
\hline 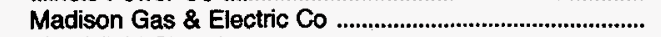 & 361 & 721 & 1,075 & 0 & 2,157 \\
\hline 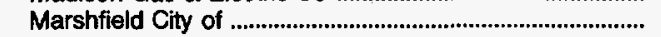 & 5 & 3 & 17 & 0 & 25 \\
\hline 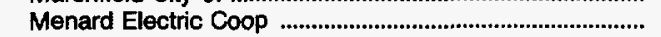 & 1 & 6 & 3 & 0 & 10 \\
\hline Plymouth City of & 0 & 0 & 0 & 15 & 15 \\
\hline 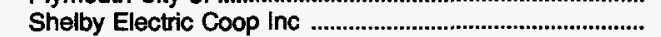 & 5 & 0 & 0 & 0 & 5 \\
\hline 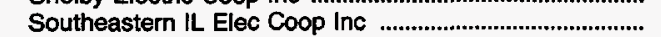 & 0 & 2 & 0 & 0 & 2 \\
\hline 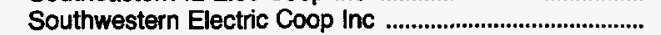 & 0 & 100 & 0 & 0 & 100 \\
\hline Springfield City of & 60 & 112 & 27 & 0 & 199 \\
\hline 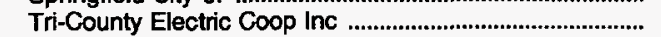 & 10 & 5 & 0 & 0 & 15 \\
\hline 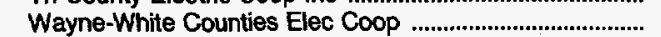 & 1 & 0 & 2 & 0 & 3 \\
\hline 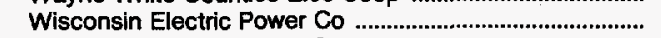 & 12,776 & 1,852 & 1,131 & 2,490 & 18,249 \\
\hline 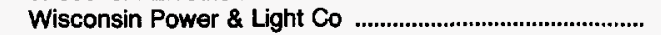 & 855 & 0 & 1,379 & 0 & 2,234 \\
\hline 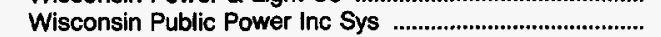 & 286 & 205 & 80 & 0 & 571 \\
\hline 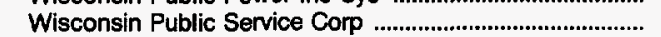 & 6,100 & 0 & 0 & $\mathbf{0}$ & 6,100 \\
\hline 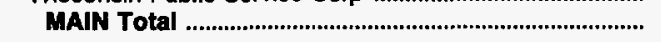 & 20,599 & 3,170 & 3,769 & 3,294 & 30,832 \\
\hline \multicolumn{6}{|l|}{ MAPP(U.S.) } \\
\hline Ames City of & 30 & 24 & 30 & 0 & 84 \\
\hline 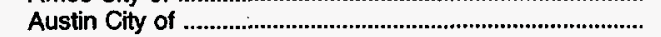 & 24 & 47 & 3 & 0 & 74 \\
\hline Barron Electric Coop & 6 & 6 & 2 & 0 & 14 \\
\hline 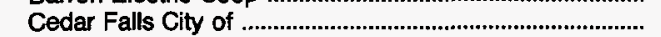 & 41 & 41 & 41 & 0 & 123 \\
\hline 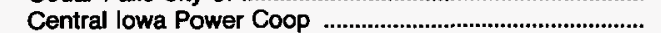 & 427 & 570 & 285 & 142 & 1,424 \\
\hline 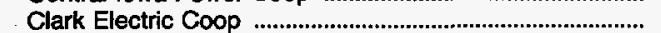 & 10 & 0 & 0 & 0 & 10 \\
\hline 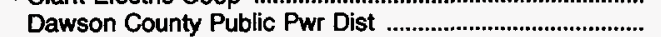 & 0 & 0 & 0 & 5 & 5 \\
\hline 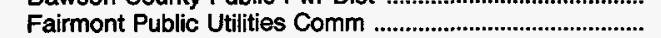 & 3 & 0 & 0 & 0 & 3 \\
\hline 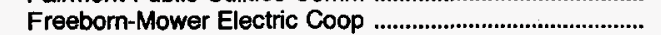 & 14 & 2 & 0 & 0 & 16 \\
\hline Grant-Lafayette Electric Coop & 25 & 20 & 10 & 0 & 55 \\
\hline IES Utilities inc & 232 & 1,080 & 566 & 478 & 2,356 \\
\hline Interstate Power Co & 196 & 147 & 147 & 0 & 490 \\
\hline 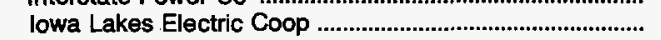 & 40 & 395 & 40 & 0 & 475 \\
\hline 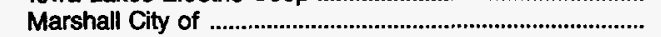 & 10 & 2 & 1 & 0 & 13 \\
\hline  & 247 & 396 & 0 & 0 & 643 \\
\hline 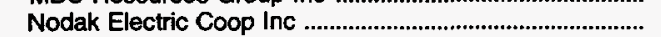 & 7 & 5 & 36 & 0 & 48 \\
\hline Midland Power Coop & 11 & 11 & 5 & 0 & 27 \\
\hline 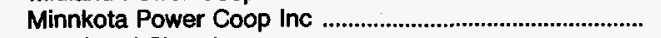 & 50 & 100 & 0 & 0 & 150 \\
\hline Moorhead City of & 78 & 19 & 0 & 0 & 97 \\
\hline 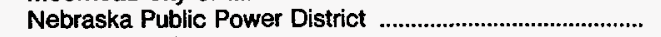 & 99 & 976 & 6 & 0 & 1,081 \\
\hline North Platte City of & 0 & 0 & 0 & 3 & 3 \\
\hline 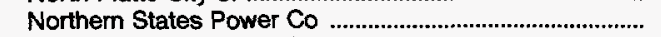 & 302 & 984 & 356 & 0 & 1,642 \\
\hline 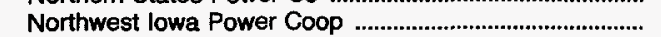 & 10 & 10 & 5 & 0 & 25 \\
\hline 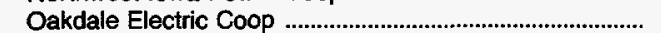 & 44 & 65 & 0 & 0 & 109 \\
\hline People's Coop Power Assn & 4 & 14 & 0 & 0 & 18 \\
\hline Pella City of & 32 & 32 & 0 & 0 & 64 \\
\hline Pierre City of & 1 & 0 & 1 & 0 & 2 \\
\hline Rochester Public Utilities & 36 & 0 & 0 & 0 & 36 \\
\hline 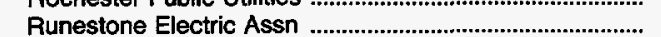 & 7 & 2 & 0 & 0 & $\begin{array}{r}30 \\
9\end{array}$ \\
\hline Superior Water Light\&Power Co & 29 & 30 & 0 & 0 & 59 \\
\hline Tri-County Electric Coop & 34 & 22 & 0 & 0 & 56 \\
\hline 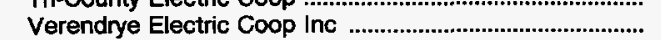 & 8 & 25 & 2 & 0 & $\begin{array}{l}56 \\
35\end{array}$ \\
\hline Vernon Electric Coop & 3 & 55 & 0 & 0 & 58 \\
\hline 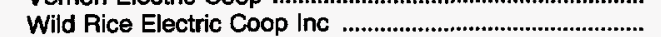 & 31 & 58 & 0 & 0 & 89 \\
\hline 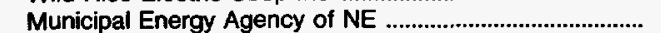 & 5 & 3 & 0 & 0 & 8 \\
\hline 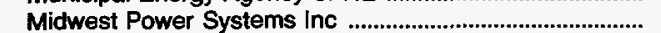 & 610 & 103 & 263 & 1,041 & 2,017 \\
\hline 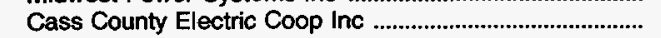 & 4 & 40 & 4 & 0 & 48 \\
\hline 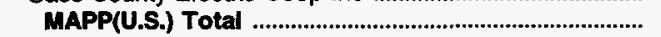 & 2,710 & 5,284 & 1,803 & 1,669 & 11,466 \\
\hline \multicolumn{6}{|l|}{ NPCC(U.S.) } \\
\hline 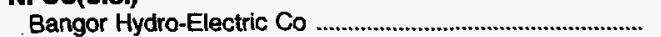 & 273 & 215 & 47 & 339 & 874 \\
\hline Blackstone Valley Electric Co & 495 & 97 & 141 & 34 & 767 \\
\hline Boston Edison Co & 7,284 & 1,127 & 2,710 & 277 & 11,398 \\
\hline Braintree Town of & 20 & 8 & 0 & 0 & 28 \\
\hline Cambridge Electric Light Co & 236 & 0 & 55 & 0 & 291 \\
\hline Central Hudson Gas \& Elec Corp & 209 & 251 & 315 & 0 & 775 \\
\hline Central Maine Power Co & 1,250 & 0 & 250 & 0 & 1,500 \\
\hline Central Vermont Pub Serv Corp & 2,465 & 0 & 618 & 0 & 3,083 \\
\hline Chicopee City of & 20 & 20 & 5 & 0 & 45 \\
\hline 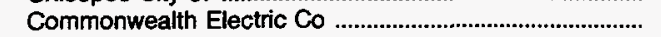 & 632 & 0 & 309 & 0 & 941 \\
\hline
\end{tabular}

See footnotes at end of table. 
Table 24. U.S. Electric Utility DSM Program Indirect Utility Costs by North American Electric Reliability Council Region and Hawaii by Cost Category, 1993 (Thousand Dollars) (Continued)

Administrative $\quad$ Marketing

\begin{tabular}{l|l|l}
\hline $\begin{array}{c}\text { Monitoring } \\
\text { and } \\
\text { Evaluation }\end{array}$ & Other $^{1}$ & $\begin{array}{c}\text { Total Indirect } \\
\text { Utility Cost }{ }^{2}\end{array}$ \\
\hline
\end{tabular}

NPCC(U.S.)

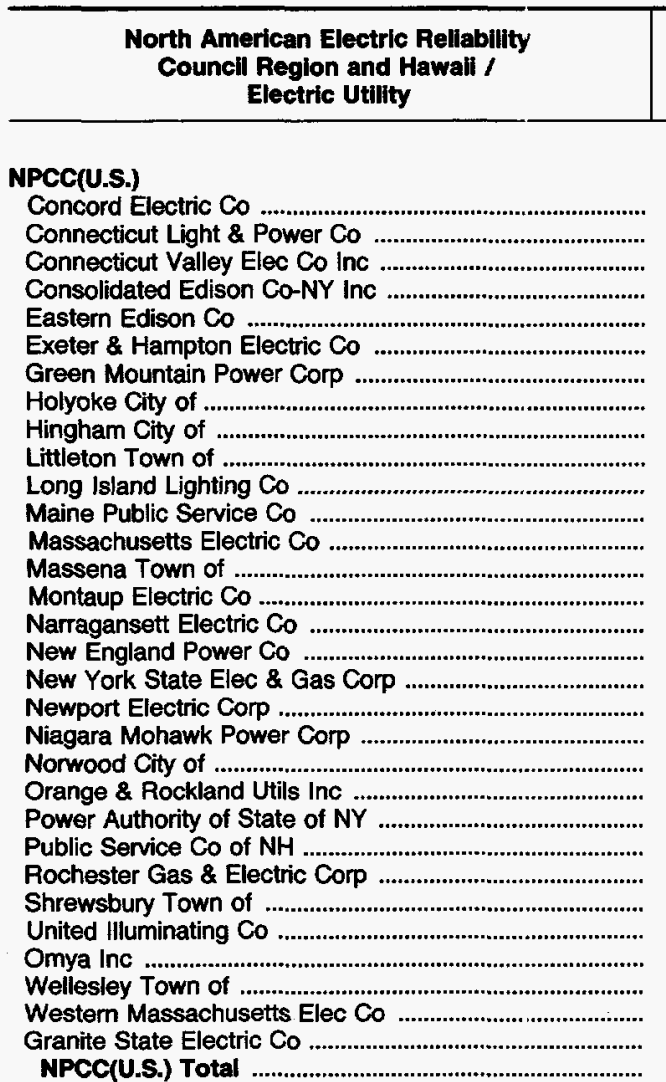

Concord Electric Co

132

112

6,916

965

117

2,231

8
0

57

2,144

24
4,718

4,718
0

1,722

1,373

120

2,857

263

14,921

1,125

1,125
830

63

0

10
0

12

0
264

252

54,836

SERC

Aiken Electric Coop Inc

Alabama Electric Coop Inc

Alabama Power Co

Albemarle City of

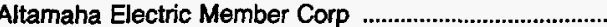

Berkeley Electric Coop Inc ...

Black River Electric Coop Inc

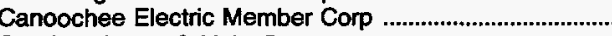

Carolina Power \& Light Co .

Carroll Electric Member Corp ...

Carteret-Crav

Central Georgia El Member Corp

Coast Electric Power Assn

Cobb Electric Membership Corp..

Coweta-Fayette El Member Corp

Crescent Electric Member Corp

Davidson Electric Member Corp

Douglas City of

Duke Power Co

Fairfield Electric Coop Inc

Flint Electric Membership Corp

Florida Keys El CoOp As

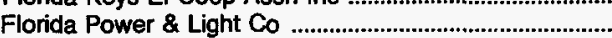

Florida Power Corp

Gainesville Regional Utilities

Greenville Utilities Comm

ric Member Corp

Jackson Electric Member Corp

Jacksonville Electric Auth

Jefferson Electric Member Corp ..

Jones-Onslow Elec Member Corp

$\begin{array}{rr}0 & 8 \\ 0 & 2,183 \\ 0 & 50 \\ 1,825 & 5,847 \\ 296 & 339 \\ 0 & 8 \\ 0 & 421 \\ 0 & 0 \\ 3 & 0 \\ 0 & 0 \\ 2,938 & 0 \\ 12 & 0 \\ 2,070 & 2,273 \\ 1 & 1 \\ 478 & 552 \\ 342 & 519 \\ 0 & 0 \\ 0 & 1,123 \\ 84 & 72 \\ 0 & 2,240 \\ 0 & 2 \\ 480 & 1,014 \\ 0 & 0 \\ 0 & 0 \\ 0 & 661 \\ 2 & 0 \\ 0 & 1,000 \\ 0 & 1 \\ 0 & 0 \\ 0 & 1,177 \\ 90 & 67 \\ 10,339 & 008\end{array}$

$\begin{array}{rr}0 & 140 \\ 718 & 3,617 \\ 0 & 162 \\ 0 & 14,588 \\ 73 & 1,673 \\ 0 & 125 \\ 1,115 & 3,767 \\ 0 & 8 \\ 0 & 3 \\ 0 & 57 \\ 717 & 5,799 \\ 79 & 115 \\ 0 & 9,061 \\ 0 & 2 \\ 129 & 2,881 \\ 0 & 2,234 \\ 0 & 120 \\ 57 & 4,037 \\ 18 & 437 \\ 0 & 17,161 \\ 0 & 2 \\ 0 & 2,619 \\ 0 & 830 \\ 0 & 63 \\ 197 & 858 \\ 0 & 12 \\ 0 & 1,000 \\ 0 & 13 \\ 18 & 18 \\ 536 & 1,977 \\ 0 & 409 \\ 4,307 & 93,490\end{array}$

140

162

4,588

125

3,767

3

5,799

2
881

2,234

120
, 037

437

2

819
830

63

858
12

1,000

13

, 977

93,490

\section{0}

293
3,105

5

110

5

240
1

7

15

18
0

419

313

0
33

2
3,537
10

34

1

0
569

6

4

50

$\begin{array}{rrr}0 & 73 & 153 \\ 5 & 0 & 408 \\ 174 & 0 & 3,279 \\ 2 & 0 & 17 \\ 1 & 0 & 3 \\ 80 & 0 & 225 \\ 0 & 0 & 35 \\ 3 & 0 & 243 \\ 0 & 0 & 19 \\ 0 & 3,700 & 3,700 \\ 3 & 0 & 17 \\ 0 & 0 & 35 \\ 0 & 0 & 46 \\ 5 & 0 & 5 \\ 0 & 0 & 590 \\ 0 & 0 & 469 \\ 0 & 0 & 3 \\ 0 & 0 & 33 \\ 1 & 0 & 5 \\ 1,303 & 15,254 & 32,144 \\ 6 & 0 & 22 \\ 0 & 0 & 471 \\ 0 & 0 & 11 \\ 0 & 0 & 11,135 \\ 0 & 347 & 4,784 \\ 0 & 0 & 356 \\ 1 & 0 & 10 \\ 0 & 0 & 134 \\ 2 & 0 & 12 \\ 22 & 0 & 119 \\ 0 & 0 & 150 \\ 0 & 0 & 9 \\ 0 & 0 & 70\end{array}$

See footnotes at end of table. 
Table 24. U.S. Electric Utility DSM Program Indirect Utility Costs by North American Electric Reliability Council Region and Hawaii by Cost Category, 1993

(Thousand Dollars) (Continued)

\begin{tabular}{c|c|c|c|c|}
\hline $\begin{array}{c}\text { North American Electric Reliability } \\
\text { Council Region and Hawail } / \\
\text { Electric Utility }\end{array}$ & Administrative & Marketing & $\begin{array}{c}\text { Monitoring } \\
\text { and } \\
\text { Evaluation }\end{array}$ & $\begin{array}{c}\text { Total Indirect } \\
\text { Utility Cost }{ }^{2}\end{array}$ \\
\hline
\end{tabular}

SERC

Kinston City of

Lakeland City of

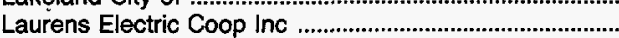

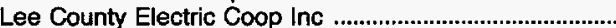

Leesburg City of

Lynches River Elec Coop Inc ............................................

Manassas City of ...

Mecklenburg Electric Coop Inc

Mid-Carolina Electric Coop Inc

Municipal Electric Authority

New Bern City of

New River Light \& Power Co ...

North Carolina Mun Power Agny .........................................

Northern Virginia Elec Coop ...

North Carolina Eastern M P A

Northern Neck Elec Coop Inc

Palmetto Electric Coop Inc

Orlando Utilities Comm

Piedmont Electric Member Corp

Prince George Electric Coop ....

Randolph Electric Member Corp

Rayle Electric Membership Corp

Reedy Creek Improvement Dist

Roanoke Electric Member Corp

Rutherford Elec Member Corp

Satilla Rural Elec Member Corp

Savannah Electric \& Power Co

Sawnee Electric Members Corp

Shenandoah Valley Elec Coop

Singing River Elec Power Assn

South Carolina Electric\&Gas Co

South Carolina Pub Serv Auth

Snapping Shoals EI Member Corp

Sumter Electric Coop Inc

Tallahassee City of

Tampa Electric Co

Tennessee Valley Authority

Thomasville City of

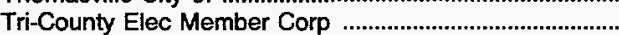

Virginia Electric \& Power Co.

Wake Electric Membership Corp

Walton Electric Member Corp

York Electric Coop Inc

Southside Electric Coop inc

Brunswick Electric Member Corp ........................................

Colquitt Electric Members Corp .

Central Electric Pwr Coop Inc

SERC Total

$\begin{array}{rr}35 & 3 \\ 107 & 24 \\ 1 & 1 \\ 114 & 0 \\ 10 & 5 \\ 3 & 8 \\ 54 & 18 \\ 23 & 0 \\ 85 & 55 \\ 13 & 0 \\ 23 & 5 \\ 2 & 3 \\ 160 & 167 \\ 20 & 68 \\ 120 & 186 \\ 5 & 5 \\ 112 & 84 \\ 172 & 391 \\ 21 & 11 \\ 1 & 0 \\ 0 & 0 \\ 2 & 0 \\ 32 & 220 \\ 32 & \end{array}$

SPP

Carroll Electric Coop Corp

Craighead Electric Coop Corp

Duncan City of

First Electric Coop Corp .........................................................

Golden Spread Elec Coop Inc

Gulf States Utilities Co

Independence City of

Oklahoma Gas \& Electric Co ..................................................

Ozark Electric Coop inc....

Petit Jean Electric Coop Corp

Red River Valley Rrl Elec Assn

South Central Ark El Coop Inc

Southwestern Electric Power Co

Verdigris Valley Elec Coop Inc

White River Valley El Coop Inc

$\begin{array}{rr}1 & 1 \\ 33 & 23 \\ 35 & 0 \\ 10 & 4 \\ 5 & 0 \\ 100 & 50 \\ 39 & 1 \\ 221 & 0 \\ 1 & 0 \\ 18 & 0 \\ 2 & 0 \\ 0 & 0 \\ 108 & 227 \\ 5 & 0 \\ 0 & 0\end{array}$

8
110
35
29
60
200
45
221
1
18
4
1
407
17
6

See footnotes at end of table. 
Table 24. U.S. Electric Utility DSM Program Indirect Utility Costs by North American Electric Reliability Council Region and Hawaii by Cost Category, 1993

(Thousand Dollars) (Continued)

\begin{tabular}{c|c|c|c|c|c|}
\hline $\begin{array}{c}\text { North American Electric Rellability } \\
\text { Council Region and Hawall / } \\
\text { Electric Utility }\end{array}$ & Administrative & $\begin{array}{c}\text { Total Indirect } \\
\text { Utillity Cost } 2\end{array}$ & $\begin{array}{c}\text { Monitoring } \\
\text { and } \\
\text { Evaluation }\end{array}$ & $\begin{array}{c}\text { Other } 1 \\
\text { Oarketing }\end{array}$ \\
\hline
\end{tabular}

SPP

Woodruff Electric Coop Corp SPP Total

Wscc(U.s.)

Alameda City of

Anaheim City of

Public Service Co

Bonneville Power Admin

Bountiful City City of

PUD No 1 of Chelan County

Colorado Springs City of

El Paso Electric Co

Forest Grove City of

Fort Collins City of

La Plata Electric Assn Inc

Longmont City of

Los Angeles City of

Loveland City of ...

Montana Power Co

Nevada Power Co

Pacific Gas \& Electric Co

Portland General Electric Co

Public Service Co of Colorado

Riverside City of

Roseville City of

Unicipal Util Dist

San Diego Gas \& Electric Co

Seattle City of

Sierra Pacific Power Co

Southern California Edison

Springfield City of

S Valley $E \mathrm{C}$ Inc

rurlock Imigation District

Black Hills Corp

Yera Irrigation District \#5

Vernon City of

Washington Water Power Co

Mohave Electric Coop Inc

WSCC(U.S.) Total

Contiguous U.S.

AsCC

Alaska Electric Light\&Power Co

Golden Valley Elec Assn Inc

AscC Total

Hawail

Maui Electric Co Ltd

Hawall Total

U.S. Total

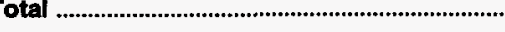

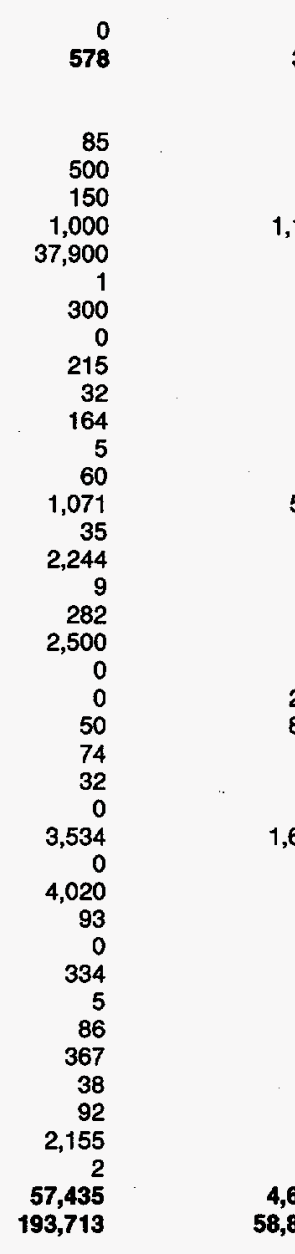

0
306

10

5

10

$\begin{array}{rr}0 & \\ 50 & 150 \\ 0 & 100 \\ 1,190 & 2,000\end{array}$

0
25
150
100

2,000

2
0

0
146
0

0
0
0

231

0
357

357
14
0

3,722
345

345
0

1,604

15
6

6
0

574

3,924

0
204

16,566

0
5

5
3

3
0
0

30

314

30,342

73,227

1,172

$\begin{array}{rr}0 & 85 \\ 0 & 57 \\ 0 & 30 \\ 0 & 2,29 \\ 0 & 39,90\end{array}$

2,290
39,900

6

300

265
361

32

164

7

65
1,835

1,835

3,388

44

282

6,222

345
250

2,590

2,590
100

38

2,387

5,723

5,857

4,020

867

22,129

334

60

89

367

122

2,469

469
17

17
103,973

453,987

128,162

$\begin{array}{rrrrr}7 & 2 & 2 & 0 & 11 \\ 199 & 39 & 0 & 0 & 238 \\ 206 & 41 & 2 & 0 & 249\end{array}$

Includes the indirect costs of demand-side management programs that cannot be mean
costs incurred in the research and development of demand-side management technologies can be attributed to one of several accounting cost categories.

Notes: -Data are final. -Data are provided for electric utilities with sales to ultimate consumers or sales for resale greater than or equal to 120,000 megawatthours.

Source: Energy Information Administration, Form ElA-861, "Annual Electric Utility Report." 

Appendix A

Technical Notes 



\section{Appendix A}

\section{Technical Notes}

\section{Source of Data}

The U.S. Electric Utility Demand-Side Management report is prepared by the Coal and Electric Data and Renewables Division; Office of Coal, Nuclear, Electric and Alternate Fuels; Energy Information Administration (EIA); U.S. Department of Energy (DOE). Data published in the U.S. Electric Utility DemandSide Management report are compiled from the Form EIA-861, "Annual Electric Utility Report" which is summarized below:

\section{Form EIA-861}

The Form EIA-861 is a mandatory census of electric utilities in the United States, its territories, and Puerto Rico. The Form EIA-861 data contained in this publication are for the United States only. The survey is used to collect information on power production and sales of electricity from approximately 3,250 electric utilities. The data collected are used to update the electric utility frame database maintained by the EIA. This database supports queries from the Executive Branch, Congress, other public agencies, and the general public. Summary data from the Form EIA-861 are also contained in the Electric Power Annual; Electric Sales and Revenue; Financial Statistics of Major U.S. Investor-Owned Electric Utilities; Financial Statistics of Major U.S. Publicly Owned Electric Utilities; Annual Energy Outlook; Electric Trade in the United States, Annual Energy Keview, Monthly Energy Review, and Electric Power Monthly. These reports present aggregate totals for electric utilities on national, State, and NERC Region levels and by ownership class and consumer class of service.

Demand-side management data are collected on Schedule V, "Demand- Side Management Information," of the Form EIA-861. Collected are data on DSM costs, annual and incremental effects for energy savings and for actual and potential peakload reductions. Also collected is information on the end use and type of energy efficiency programs. Demandside management data collected on the Form EIA-861 are estimated by electric utilities based on engineering data, statistical analysis, or other estimation methods. The form was revised for the 1993 data collection to collect information on estimation methodologies used by utilities to derive DSM data and the methods used for verification of the estimated energy effects.

EIA collects information on DSM activities from all utilities with DSM programs. DSM data are aggregated at the NERC region and consumer sector levels. Utilities with sales to ultimate consumers or sales for resale greater than or equal to 120,000 megawatthours report incremental peakload reductions and energy savings for the reporting year, annual peakload reductions and energy savings for the reporting year and first- and fifth-forecast years, itemized direct and indirect utility costs and nonutility cost attributable to DSM programs for all 3 years, end use and type of energy efficiency programs, and evaluation and verification data. Annual and incremental effects for the reporting year are reported by consumer sector (residential, commercial, industrial, other) for each program category (energy efficiency, direct load control, interruptible load, other load management, other DSM programs, and load building). Forecast peakload reductions and energy savings are reported by program category with all consumer sectors combined. Utilities with sales to ultimate consumers and sales for resale less than 120,000 megawatthours report selected items: incremental peakload reductions and energy savings, total utility cost, total nonutility cost, and total DSM cost for the reporting year and first- and fifth-forecast years, end use and type of energy efficiency programs, and evaluation and verification data. In years prior to 1992, utilities with sales less than 120,000 megawatthours did not report on DSM activities.

Instrument and Design History. The Form EIA-861 was implemented in January 1985 to collect data as of year-end 1984. Schedule V, "Demand-Side Management Information," was added to the survey in 1990 to collect data for year-end 1989. Schedule V was revised for the 1991 collection and again for the 1993 year-end collection. The Federal Administration Act of 1974 (Public Law 93-275) and the Energy Policy Act of 1992 define the legislative authority to collect these data.

Data Processing. The Form EIA- 861 is mailed to the respondents in January to collect data as of the end of the preceding calendar year. The completed forms are to be returned to the EIA by May 1 . Internal edit checks are performed to verify that current data are comparable to data reported the previous year. 
Respondents are telephoned to obtain clarification of reported data and to obtain missing data.

\section{Quality of Data}

\section{Voltage Reduction.}

Voltage reduction, though not considered a DSM program, may be used by utilities to reduce load since power provided to the consumers is a function of both voltage and current. Voltage reduction is mainly used in emergency situations, although some utilities use it to reduce demand during peakload periods under normal operating conditions.

During normal operating conditions, utilities provide service to retail consumers within a range of voltages (e.g., $120 \mathrm{v} \mp 5$ percent). States generally promulgate rules that describe the service utilities must provide to customers, including voltage levels. During emergency situations, utilities are allowed to go beyond the normal operating range to a limited extent. Most systems that use voltage reduction during emergencies limit the variation to a maximum of 5 percent outside of normal operating limits, but some go as high as 8 percent. The reduction applied may be any level up to the maximum, depending on the circumstances. Although the emergency voltage reductions go outside of the normal ranges, they are implemented for short periods of time (as little as 10 minutes to an hour). Voltage reduction is effected by reducing the voltage at customer-level substations (distribution system), either manually or remotely, if the utility system is fully automated. A voltage reduction can be made for one area of a utility's service territory, or for an entire utility system.

The amount of power that is saved when voltage is reduced depends on many factors including the types of load and the relative proportions of those loads at the time the voltage is reduced. Since load mix and level varies by season and time of day, the impacts of voltage reduction will vary accordingly. The potential peakload savings that may be achieved under a set of specific circumstances for a 5 percent reduction in voltage, can range from negligible to 5 percent of summer peak load, with most savings being less than 3 percent of winter or summer peak load.

Some utilities also use the term "voltage reduction" to include improvements in their distribution system that allow them to operate at lower nominal voltages. By investing in improved voltage regulators, line reconductoring, and other distribution equipment, utilities can lower substation operating voltage and still provide customers with adequate voltage, thereby saving energy. When the savings are adequate to justify the investment, utilities may implement such a program and refer to it as voltage reduction or conservation voltage reduction.
The Office of Coal, Nuclear, Electric and Alternate Fuels (CNEAF) is responsible for routine data improvement and quality assurance activities. All operations in this office are done in accordance with formal standards established by the EIA. These standards are the measuring rod necessary for quality statistics. Data improvement efforts include verification of data-keyed input by automatic computerized methods, editing by subject matter specialists, and follow up on nonrespondents. The CNEAF office supports the quality assurance efforts of the data collectors by providing advisory reviews of the structure of information requirements and of proposed designs for new and revised data collection forms and systems. Once implemented, the actual performance of working data collection systems is also validated. Computerized respondent data files are checked to identify those who fail to respond to the survey. By law, nonrespondents may be fined or otherwise penalized for not filing a mandatory EIA data form. Before invoking the law, the EIA tries to obtain the required information by encouraging cooperation of nonrespondents.

Completed forms received by the CNEAF office are sorted, screened for completeness of reported information, and keyed onto computer tapes for storage and transfer to random access data bases for computer processing. The information coded on the computer tapes is manually spot-checked against the forms to certify accuracy of the tapes. To ensure the quality standards established by the EIA, formulas that use the past history of data values in the data base have been designed and implemented to check data input for errors automatically. Data values that fall outside the ranges prescribed in the formulas are verified by telephoning respondents to resolve any discrepancies.

\section{Data Editing System}

Data from the form surveys are edited using automated systems. The edit includes both deterministic checks, in which records are checked for the presence of required fields and their validity; and statistical checks, in which estimation techniques are used to validate data according to their behavior in the past and in comparison to other current fields.

\section{Confidentiality of the Data}

The data collected on the Form EIA-861 used for input to this report are not confidential. 


\section{Rounding Rules for Data}

Given a number with $\mathrm{r}$ digits to the left of the decimal and $\mathrm{d}+\mathrm{t}$ digits in the fraction part, with $\mathrm{d}$ being the place to which the number is to be rounded and $t$ being the remaining digits which will be truncated, this number is rounded to $\mathrm{r}+\mathrm{d}$ digits by adding 5 to the $(r+d+1)$ th digit when the number is positive or by subtracting 5 when the number is negative. The $t$ digits are then truncated at the $(r+d+1)$ th digit. The symbol for a rounded number truncated to zero is $\left({ }^{*}\right)$.

\section{Percent Difference Calculation}

The following formula is used to calculate percent differences.

Percent Difference $=\left(\frac{x\left(t_{2}\right)-x\left(t_{1}\right)}{x\left(t_{1}\right)}\right) \times 100$, where $x\left(t_{1}\right)$ and $x\left(t_{2}\right)$ denote the quantity at year $t_{1}$ and subsequent year $t_{2}$.

\section{CNEAF Data Revision and Policy}

The Office of Coal, Nuclear, Electric and Alternate Fuels has adopted the following policy with respect to the revision and correction of recurrent data in energy publications:

1. Annual survey data collected by this office are published either as preliminary or final when first appearing in a data report. Data initially released as preliminary will be so noted in the report. These data will be revised, if necessary, and declared final in the next publication of the data.

2. The magnitude of changes due to revisions experienced in the past will be included in the data reports, so that the reader can assess the accuracy of the data.

3. After data are published as final, corrections will be made only in the event of a greater than one percent difference at the national level. Corrections for differences that are less than the before-mentioned threshold are left to the discretion of the Office Director.

The U.S. Electric Utility Demand-Side Management (DSM) report presents the most current annual data available to the EIA. The statistics may differ from those published previously in EIA publications due to corrections, revisions, or other adjustments to the data subsequent to its original release. The status (preliminary versus final) of DSM data published by EIA follows:

- U.S. Electric Utility Demand-Side Management Data on demand-side management from the Form EIA-861 for 1993 and previous years are final. A comparison of preliminary versus final data at the national level for 1993 will be provided in the Electric Power Annual 1994.

- Electric Power Annual 1993

The chapter in the Electric Power Annual for DSM contains data on demand-side management from the Form EIA-861 for 1993 that are preliminary. Data for previous years are final.

Corrections were made to data for 1989, 1990, 1991 and 1992 that previously was published as final in the Electric Power Annual 1993. These corrections are contained in Table A2.

\section{Use of the Glossary}

The terms in the glossary have been defined for general use. Restrictions on the definitions as used in these data collection systems are included in each definition when necessary to define the terms as they are used in this report.

\section{Obtaining Copies of Data}

The data are available on machine-readable tapes. Tapes may be purchased by using Visa, MasterCard, or American Express cards as well as money orders or checks payable to the National Technical Information Service (NTIS). Purchasers may also use NTIS and Government Printing Office depository accounts. To place an order, contact:

National Technical Information Service (NTIS)

Office of Data Base Services

U.S. Department of Commerce

5285 Port Royal Road

Springfield, Virginia 22161

(703) $487-4650$

The data for 1991, 1992, and 1993 filed on the Form EIA-861 are also available on the Internet in compressed format through FTP at ftp.fedworld.gov, or through use of a world-wide-web browser such as Mosaic at www.fedworld.gov, in the /pub/energy subdirectory. 
Table A1. Unit-of-Measure Equivalents

\begin{tabular}{|c|c|c|c|}
\hline Unit & & Equivalent & \\
\hline $\begin{array}{l}\text { Kilowatt (kW) } \\
\text { Megawatt (MW) } \\
\text { Gigawatt (GW) } \\
\text { Terawatt (TW) }\end{array}$ & $\begin{array}{r}1,000 \\
1,000,000 \\
1,000,000,000 \\
1,000,000,000,000\end{array}$ & $\begin{array}{l}\text { (One Thousand) } \\
\text { (One Million) } \\
\text { (One Billion) } \\
\text { (One Trillion) }\end{array}$ & $\begin{array}{l}\text { Watts } \\
\text { Watts } \\
\text { Watts } \\
\text { Watts }\end{array}$ \\
\hline Gigawatt Thousand Gigawatts & $\begin{array}{r}1,000,000 \\
1,000,000,000\end{array}$ & $\begin{array}{l}\text { (One Million) } \\
\text { (One Billion) }\end{array}$ & $\begin{array}{l}\text { Kilowatts } \\
\text { Kilowatts }\end{array}$ \\
\hline $\begin{array}{l}\text { Kilowatthours (kWh) } \\
\text { Megawatthours (MWh) } \\
\text { Gigawatthours }(\mathrm{GWh}) \\
\text { Terawatthours }(\mathrm{TWh})\end{array}$ & $\begin{array}{r}1,000 \\
1,000,000 \\
1,000,000,000 \\
1,000,000,000,000\end{array}$ & $\begin{array}{l}\text { (One Thousand) } \\
\text { (One Million) } \\
\text { (One Billion) } \\
\text { (One Trillion) }\end{array}$ & $\begin{array}{l}\text { Watthours } \\
\text { Watthours } \\
\text { Watthours } \\
\text { Watthours }\end{array}$ \\
\hline $\begin{array}{l}\text { Gigawatthours } \\
\text { Thousand Gigawatthours }\end{array}$ & $\begin{array}{r}1,000,000 \\
1,000,000,000\end{array}$ & $\begin{array}{l}\text { (One Million) } \\
\text { (One Billion) }\end{array}$ & $\begin{array}{l}\text { Kilowatthours } \\
\text { Kilowatthours }\end{array}$ \\
\hline
\end{tabular}

Source: Energy Information Administration, Coal and Electric Data and Renewables Division.

Table A2. Corrections to DSM Data, 1989 through 1992

\begin{tabular}{|c|c|c|c|c|c|c|c|c|}
\hline & 1989 & \multicolumn{2}{|c|}{1990} & \multicolumn{3}{|c|}{1991} & \multicolumn{2}{|c|}{1992} \\
\hline Utility & $\begin{array}{l}\text { Energy } \\
\text { Savings } \\
\text { (MWh) }\end{array}$ & $\begin{array}{l}\text { Energy } \\
\text { Savings } \\
\text { (MWh) }\end{array}$ & $\begin{array}{c}\text { Actual } \\
\text { Peakload } \\
\text { Reduction } \\
(\mathbf{k W})\end{array}$ & $\begin{array}{l}\text { Energy } \\
\text { Savings } \\
\text { (MWh) }\end{array}$ & $\begin{array}{c}\text { Actual } \\
\text { Peakload } \\
\text { Reduction } \\
(\mathbf{k W})\end{array}$ & $\begin{array}{c}\text { Cost } \\
(\$ 1000)\end{array}$ & $\begin{array}{l}\text { Energy } \\
\text { Savings } \\
\text { (MWh) }\end{array}$ & $\begin{array}{c}\text { Cost } \\
(\$ 1000)\end{array}$ \\
\hline $\begin{array}{l}\text { Bonneville Power } \\
\text { Administration }\end{array}$ & $-2,054,763$ & $-1,649,639$ & -. & $-1,599,567$ & -- & $-55,840$ & $-1,830,108$ & $-104,824$ \\
\hline $\begin{array}{l}\text { Carolina Power } \\
\text { and Light }\end{array}$ & $3,651,234$ & $3,578,860$ & $1,068,000$ & $4,417,700$ & $1,120,000$ & -- & -- & -- \\
\hline $\begin{array}{l}\text { Southern } \\
\text { California Edison }\end{array}$ & -- & $-3,717,000$ & -- & $-4,324,000$ & -- & -- & -- & -- \\
\hline Other $^{1}$ & -- & -- & -- & -- & - & -- & $2,431,108$ & -- \\
\hline Total & $\mathbf{1 , 5 9 6 , 4 7 1}$ & $-1,787,779$ & $1,068,000$ & $-1,505,867$ & $1,120,000$ & $-55,840$ & 601,000 & $-104,824$ \\
\hline
\end{tabular}

'Other includes, City of Ashland, PUD No. 1 of Benton County, Canby Utility Board, PUD No. 1 of Chelan County, City of Eugene, City of Forest Grove, PUD No. 1 of Lewis County, PUD No. 2 of Grant County, City of Port Angeles, Salem Electric Cooperative, PUD No. 1 of Snohomish County, City of Tacoma, Vera Irrigation District No. 15, and Columbia River Peoples Utility District.

Source: Energy Information Administration, Form ELA-861, "Annual Electric Utility Report." 
Appendix B

Glossary 



\section{Glossary}

Actual Peakload Reduction: The actual reduction in annual peak load (measured in kilowatts) achieved by consumers that participate in a utility DSM program. It reflects the real changes in the demand for electricity resulting from a utility DSM program that is in effect at the same time the utility experiences its annual peak load, as opposed to the installed peakload reduction capability (i.e., Potential Peakload Reduction). It should account for the regular cycling of energy efficient units during the period of annual peak load.

Annual Effects: The total effects in energy use (measured in megawatthours) and peak load (measured in kilowatts) caused by all participants in the DSM programs that are in effect during a given year. It includes new and existing participants in existing programs (those implemented in prior years that are in place during the given year) and all participants in new programs (those implemented during the given year). The effects of new participants in existing programs and all participants in new programs should be based on their start-up dates (i.e., if participants enter a program in July, only the effects from July to December should be ?reported). If start-up dates are unknown and cannot be reasonably estimated, the effects can be annualized (i.e., assume the participants were initiated into the program on January 1 of the given year). The Annual Effects should consider the useful life of efficiency measures, by accounting for building demolition, equipment degradation and attrition.

Appliances: Energy Efficiency program promotion of high efficiency appliances such as dishwashers, ranges, refrigerators, and freezers in the residential, commercial, and industrial sectors. Includes programs aimed at improving the efficiency of refrigeration equipment and electrical cooking equipment, including replacement. It also includes the promotion and identification of high efficiency appliances in retail stores using a labeling system different from the Federally-mandated Energy Guide. Energy Efficiency program promotion of high efficiency cooling and heating appliances are included under Cooling System and Heating System, respectively.

Asset: An economic resource, tangible or intangible, which is expected to provide benefits to a business.

Average Revenue per Kilowatthour: The average revenue per kilowatthour of electricity sold by sector (residential, commercial, industrial, or other) and geographic area (State, Census division, and national), is calculated by dividing the total monthly revenue by the corresponding total monthly sales for each sector and geographic area.

Baseload: The minimum amount of electric power delivered or required over a given period of time at a steady rate.

Baseload Capacity: The generating equipment normally operated to serve loads on an around-theclock basis.

Baseload Plant: A plant, usually housing highefficiency steam-electric units, which is normally operated to take all or part of the minimum load of a system, and which consequently produces electricity at an essentially constant rate and runs continuously. These units are operated to maximize system mechanical and thermal efficiency and minimize system operating costs.

Capacity (Purchased): The amount of energy and capacity available for purchase from outside the system.

Capacity Charge: An element in a two-part pricing method used in capacity transactions (energy charge is the other element). The capacity charge, sometimes called Demand Charge, is assessed on the amount of capacity being purchased.

Census Divisions: The nine geographic divisions of the United States established by the Bureau of the Census, U.S. Department of Commerce, for the purpose of statistical analysis. The boundaries of Census divisions coincide with State boundaries. The Pacific Division is subdivided into the Pacific Contiguous and Pacific Noncontiguous areas.

Cogenerator: A generating facility that produces electricity and another form of useful thermal energy (such as heat or steam), used for industrial, commercial, heating, or cooling purposes. To receive status as a qualifying facility (QF) under the Public Utility Regulatory Policies Act (PURPA), the facility must produce electric energy and "another form of useful thermal energy through the sequential use of energy," and meet certain ownership, operating, and efficiency criteria established by the Federal Energy Regulatory 
Commission (FERC). (See the code of Federal Regulations, Title 18, Part 292.)

Coincidental Peak Load: The sum of two or more peak loads that occur in the same time interval.

Commercial: The commercial sector is generally defined as nonmanufacturing business establishments, including hotels, motels, restaurants, wholesale businesses, retail stores, and health, social, and educational institutions. The utility may classify commercial service as all consumers whose demand or annual use exceeds some specified limit. The limit may be set by the utility based on the rate schedule of the utility.

Commercial Operation: Commercial operation begins when control of the loading of the generator is turned over to the system dispatcher.

Conservation and Other DSM: This Demand-Side Management category represents the amount of consumer peakload reduction at the time of system peak due to utility programs that reduce consumer load during many hours of the year. Examples include utility rebate and shared savings activities for the installation of energy efficient appliances, lighting and electrical machinery, and weatherization materials. In addition, this category includes all other DemandSide Management activities, such as thermal storage, time-of-use rates, fuel substitution, measurement and evaluation, and any other utility-administered Demand-Side Management activity designed to reduce demand and/or electricity use.

Cooling System: Energy Efficiency program promotion aimed at improving the efficiency of the cooling delivery system, including replacement, in the residential, commercial, or industrial sectors.

Cooperative Electric Utility: An electric utility legally established to be owned by and operated for the benefit of those using its service. The utility company will generate, transmit, and/or distribute supplies of electric energy to a specified area not being serviced by another utility. Such ventures are generally exempt from Federal income tax laws. Most electric cooperatives have been initially financed by the Rural Electrification Administration, U.S. Department of Agriculture.

Cost: The amount paid to acquire resources, such as plant and equipment, fuel, or labor services.

Demand (Electric): The rate at which electric energy is delivered to or by a system, part of a system, or piece of equipment, at a given instant or averaged over any designated period of time.

Demand-Side Management: The planning, implementation, and monitoring of utility activities designed to encourage consumers to modify patterns of electricity usage, including the timing and level of electricity demand. It refers only to energy and load-shape modifying activities that are undertaken in response to utility-administered programs. It does not refer to energy and load-shape changes arising from the normal operation of the marketplace or from government-mandated energy-efficiency standards. Demand-Side Management (DSM) covers the complete range of load-shape objectives, including strategic conservation and load management, as well as strategic load growth.

Demand-Side Management Cost: The cost incurred by the utility to achieve the capacity and energy savings from the Demand-Side Management Program. Costs (expenditures) incurred by consumers or third parties are to be excluded. The costs are to be reported in nominal dollars in the year in which they are incurred, regardless of when the savings occur. Program costs include expensed items incurred to implement the program, incentive payments provided to consumers to install Demand-Side Management measures, and annual operation and maintenance expenses incurred during the year. Utility costs that are general, administrative, or not specific to a particular Demand-Side Management category are to be included in "other" costs.

Direct Load Control: Refers to program activities that can interrupt consumer load at the time of annual peak load by direct control of the utility system operator by interrupting power supply to individual appliances or equipment on consumer premises. This type of control usually involves residential consumers. Direct Load Control excludes Interruptible Load and Other Load Management effects. (Direct Load Control, as defined here, is synonymous with Direct Load Control Management reported to the North American Electric Reliability Council on the voluntary Office of Energy Emergency Operations Form OE-411, "Coordinated Regional Bulk Power Supply Program Report," with the exception that annual peakload effects are reported here and seasonal (i.e., summer and winter) peakload effects are reported on the OE-411.)

Direct Utility Cost: A utility cost that is identified with one of the DSM program categories (i.e. Energy Efficiency, Direct Load Control, Interruptible Load, Other Load Management, Other DSM Programs, Load Building).

Electric Plant (Physical): A facility containing prime movers, electric generators, and auxiliary equipment for converting mechanical, chemical, and/or fission energy into electric energy.

Electric Rate Schedule: A statement of the electric rate and the terms and conditions governing its application, including attendant contract terms and conditions that have been accepted by a regulatory body with appropriate oversight authority.

Electric Utility: A corporation, person, agency, authority, or other legal entity or instrumentality that owns and/or operates facilities within the United States, its territories, or Puerto Rico for the generation, transmission, distribution, or sale of electric energy primarily for use by the public and files forms listed in the Code of Federal Regulations, Title 18, Part 141. Facilities that qualify as cogenerators or 
small power producers under the Public Utility Kegulatory Policies Act (PURPA) are not considered electric utilities.

Energy: The capacity for doing work as measured by the capability of doing work (potential energy) or the conversion of this capability to motion (kinetic energy). Energy has several forms, some of which are easily convertible and can be changed to another form useful for work. Most of the world's convertible energy comes from fossil fuels that are burned to produce heat that is then used as a transfer medium to mechanical or other means in order to accomplish tasks. Electrical energy is usually measured in kilowatthours, while heat energy is usually measured in British thermal units.

Energy Charge: That portion of the charge for electric service based upon the electric energy ( $\mathrm{kWh}$ ) consumed or billed.

Energy Deliveries: Energy generated by one electric utility system and delivered to another system through one or more transmission lines.

Energy Effects: The changes in aggregate electricity use (measured in megawatthours) for customers that participate in a utility DSM program. Energy Effects should represent changes at the consumer meter (i.e. exclude transmission and distribution effects) and reflect only activities that are undertaken specifically in response to utility-administered programs, including those activities implemented by third parties under contract to the utility. To the extent possible, Energy Effects should exclude non-program related effects such as changes in energy usage attributable to nonparticipants, government-mandated energy-efficiency standards that legislate improvements in building and appliance energy usage, changes in consumer behavior that result in greater energy use after initiation in a DSM program, the natural operations of the marketplace, and weather and business-cycle adjustments.

Energy Efficiency: Refers to programs that are aimed at reducing the energy used by specific end-use devices and systems, typically without affecting the services provided. These programs reduce overall electricity consumption (reported in megawatthours), often without explicit consideration for the timing of program-induced savings. Such savings are generally achieved by substituting technically more advanced equipment to produce the same level of end-use services (e.g. lighting, heating, motor drive) with less electricity. Examples include high-efficiency appliances, efficient lighting programs, high-efficiency heating, ventilating and air conditioning (HVAC) systems or control modifications, efficient building design, advanced electric motor drives, and heat recovery systems.

Energy Receipts: Energy generated by one electric utility system and received by another system through one or more transmission lines.
Energy Source: 'The primary source that provides the power that is converted to electricity through chemical, mechanical, or other means. Energy sources include coal, petroleum and petroleum products, gas, water, uranium, wind, sunlight, geothermal, and other sources.

Expenditure: The incurrence of a liability to obtain an asset or service.

Facility: An existing or planned location or site at which prime movers, electric generators, and/or equipment for converting mechanical, chemical, and/or nuclear energy into electric energy are situated, or will be situated. A facility may contain more than one generator of either the same or different prime mover type. For a cogenerator, the facility includes the industrial or commercial process.

Federal Energy Regulatory Commission (FERC): A quasi-independent regulatory agency within the Department of Energy having jurisdiction over interstate electricity sales, wholesale electric rates, hydroelectric licensing, natural gas pricing, oil pipeline rates, and gas pipeline certification.

Federal Power Commission: The predecessor agency of the Federal Energy Regulatory Commission. The Federal Power Commission (FPC) was created by an Act of Congress under the Federal Water Power Act on June 10, 1920. It was charged originally with regulating the electric power and natural gas industries. The FPC was abolished on September 20, 1977, when the Department of Energy was created. The functions of the FPC were divided between the Department of Energy and the Federal Energy Regulatory Commission.

FERC: The Federal Energy Regulatory Commission.

Firm Power: Power or power-producing capacity intended to be available at all times during the period covered by a guaranteed commitment to deliver, even under adverse conditions.

Forced Outage: The shutdown of a generating unit, transmission line or other facility, for emergency reasons or a condition in which the generating equipment is unavailable for load due to unanticipated breakdown.

Generating Unit: Any combination of physically connected generator(s), reactor(s), boiler(s), combustion turbine(s), or other prime mover(s) operated together to produce electric power.

Generation (Electricity): The process of producing electric energy by transforming other forms of energy; also, the amount of electric energy produced, expressed in watthours (Wh).

Gross Generation: The total amount of electric energy produced by the generating units at a generating station or stations, measured at the generator terminals. 
Net Generation: Gross generation less the electric energy consumed at the generating station for station use.

Generator: A machine that converts mechanical energy into electrical energy.

Generator Nameplate Capacity: The full-load continuous rating of a generator, prime mover, or other electric power production equipment under specific conditions as designated by the manufacturer. Installed generator nameplate rating is usually indicated on a nameplate physically attached to the generator.

Grid: The layout of an electrical distribution system.

Gross Generation: The total amount of electric energy produced by a generating facility, as measured at the generator terminals.

Heating System: Energy Efficiency program promotion aimed at improving the efficiency of the heating delivery system, including replacement, in the residential, commercial, or industrial sectors.

Incremental Effects: The annual effects in energy use (measured in megawatthours) and peak load (measured in kilowatts) caused by new participants in existing DSM programs and all participants in new DSM programs during a given year. Reported Incremental Effects should be annualized to indicate the program effects that would have occurred had these participants been initiated into the program on January 1 of the given year. Incremental effects are not simply the Annual Effects of a given year minus the Annual Effects of the prior year, since these net effects would fail to account for program attrition, degradation, demolition, and participant dropouts.

Indirect Utility Cost: A utility cost that may not be meaningfully identified with any particular DSM program category. Indirect costs could be attributable to one of several accounting cost categories (i.e., Administrative, Marketing, Monitoring \& Evaluation, Utility-Earned Incentives, Other). Accounting costs that are known DSM program costs should not be reported under Indirect Utility Cost, rather those costs should be reported as Direct Utility Costs under the appropriate DSM program category.

Industrial: The industrial sector is generally defined as manufacturing, construction, mining agriculture, fishing and forestry establishments Standard Industrial Classification (SIC) codes 01-39. The utility may classify industrial service using the SIC codes, or based on demand or annual usage exceeding some specified limit. The limit may be set by the utility based on the rate schedule of the utility.

Interruptible Load: Refers to program activities that, in accordance with contractual arrangements, can interrupt consumer load at times of seasonal peak load by direct control of the utility system operator or by action of the consumer at the direct request of the system operator. It usually involves commercial and industrial consumers. In some instances the load reduction may be affected by direct action of the system operator (remote tripping) after notice to the consumer in accordance with contractual provisions. For example, loads that can be interrupted to fulfill planning or operation reserve requirements should be reported as Interruptible Load. Interruptible Load as defined here excludes Direct Load Control and Other Load Management. (Interruptible Load, as reported here, is synonymous with Interruptible Demand reported to the North American Electric Reliability Council on the voluntary Office of Energy Emergency Operations Form OE-411, "Coordinated Regional Bulk Power Supply Program Report," with the exception that annual peakload effects are reported on the Form EIA-861 and seasonal (i.e., summer and winter) peakload effects are reported on the OE-411).

Kilowatt (kW): One thousand watts.

Kilowatthour (kWh): One thousand watthours.

Liability: An amount payable in dollars or by future services to be rendered.

Load (Electric): The amount of electric power delivered or required at any specific point or points on a system. The requirement originates at the energyconsuming equipment of the consumers.

Load Building: Refers to programs that are aimed at increasing the usage of existing electric equipment or the addition of electric equipment. Examples include industrial technologies such as induction heating and melting, direct arc furnaces and infrared drying; cooking for commercial establishments; and heat pumps for residences. Load Building should include programs that promote electric fuel substitution. Load Building effects should be reported as a negative number, shown with a minus sign.

Marketing Cost: Expenses directly associated with the preparation and implementation of the strategies designed to encourage participation in a DSM program. The category excludes general market and load research costs.

Monitoring \& Evaluation Cost: Expenditures associated with the planning, collection, and analysis of data used to assess program operation and effects. It includes the activities such as load metering, customer surveys, new technology testing, and program evaluations that are intended to establish or improve the ability to monitor and evaluate the impacts of DSM programs, collectively or individually.

Maximum Demand: The greatest of all demands of the load that has occurred within a specified period of time.

Megawatt (MW): One million watts.

Megawatthour (MWh): One million watthours. 
Net Capability: The maximum load-carrying ability of the equipment, exclusive of station use, under specified conditions for a given time interval, independent of the characteristics of the load. (Capability is determined by design characteristics, physical conditions, adequacy of prime mover, energy supply, and operating limitations such as cooling and circulating water supply and temperature, headwater and tailwater elevations, and electrical use.)

Net Generation: Gross generation minus plant use from all electric utility owned plants. The energy required for pumping at a pumped-storage plant is regarded as plant use and must be deducted from the gross generation.

Net Summer Capability: The steady hourly output, which generating equipment is expected to supply to system load exclusive of auxiliary power, as demonstrated by tests at the time of summer peak load.

Net Winter Capability: The steady hourly output which generating equipment is expected to supply to system load exclusive of auxiliary power, as demonstrated by tests at the time of winter peak load.

New Construction: Energy-efficiency program promotion to encourage the building of new homes, buildings, and plants to exceed standard governmentmandated energy efficiency codes; it may include major renovations of existing facilities.

Noncoincidental Peak Load: The sum of two or more peak loads on individual systems that do not occur in the same time interval. Meaningful only when considering loads within a limited period of time, such as a day, week, month, a heating or cooling season, and usually for not more than 1 year.

North American Electric Reliability Council (NERC): A council formed in 1968 by the electric utility industry to promote the reliability and adequacy of bulk power supply in the electric utility systems of North America. NERC consists of ten regional reliability councils and encompasses essentially all the power regions of the contiguous United States, Canada, and Mexico. The NERC Regions are:

ASCC - Alaskan System Coordination Council

ECAR - East Central Area Reliability Coordination Agreement

ERCOT - Electric Reliability Council of Texas

MAIN - Mid-America Interconnected Network

MAAC - Mid-Atlantic Area Council

MAPP - Mid-Continent Area Power Pool

NPCC - Northeast Power Coordinating Council

SERC - Southeastern Electric Reliability Council

SPP - Southwest Power Pool

WSCC - Western Systems Coordinating Council

Other Cost: A residual category to capture the Indirect Costs of DSM programs that cannot be meaning- fully included in any of the other cost categories listed and defined herein. Included are costs such as those incurred in the research and development of DSM technologies.

Other DSM Programs: A residual category to capture the effects of DSM programs that cannot be meaningfully included in any of the program categories listed and defined herein. The energy effects attributable to this category should be the net effects of all the residual programs. Programs that promote consumer's substitution of electricity by other energy types should be included in Other DSM Programs. Also, self-generation should be included in Other DSM Programs to the extent that it is not accounted for as backup generation in Other Load Management or Interruptible Load categories.

Other Incentives: Energy Efficiency programs that offer cash or noncash awards to electric energy efficiency deliverers, such as appliance and equipment dealers, building contractors, and architectural and engineering firms, that encourage consumer participation in a DSM program and adoption of recommended measures.

Other Load Management: Refers to programs other than Direct Load Control and Interruptible Load that limit or shift peak load from on-peak to off-peak time periods. It includes technologies that primarily shift all or part of a load from one time-of-day to another and secondarily may have an impact on energy consumption. Examples include space heating and water heating storage systems, cool storage systems, and load limiting devices in energy management systems. This category also includes programs that aggressively promote time-of-use (TOU) rates and other innovative rates such as real time pricing. These rates are intended to reduce consumer bills and shift hours of operation of equipment from on-peak to off-peak periods through the application of time-differentiated rates.

Outage: The period during which a generating unit, transmission line, or other facility is out of service.

Peak Demand: The maximum load during a specified period of time.

Peaking Capacity: Capacity of generating equipment normally reserved for operation during the hours of highest daily, weekly, or seasonal loads. Some generating equipment may be operated at certain times as peaking capacity and at other times to serve loads on an around-the-clock basis.

Percent Difference: The relative change in a quantity over a specified time period. It is calculated as follows: the current value has the previous value subtracted from it; this new number is divided by the absolute value of the previous value; then this new number is multiplied by 100 .

Planned Generator: A proposal by a company to install electric generating equipment at an existing or planned facility or site. The proposal is based on the 
owner having obtained (1) all environmental and regulatory approvals, (2) a signed contract for the electric energy, or (3) financial closure for the facility.

Potential Peakload Reduction: The amount of annual peakload reduction capability (measured in kilowatts) that can be deployed from Direct Load Control, Interruptible Load, Other Load Management, and Other DSM Program activities. It represents the load that can be reduced either by the direct control of the utility system operator or by the consumer in response to a utility request to curtail load. It reflects the installed load reduction capability, as opposed to the Actual Peak Reduction achieved by participants, during the time of annual system peak load.

Power: The rate at which energy is transferred. Electrical energy is usually measured in watts. Also used for a measurement of capacity.

Power Pool: An association of two or more interconnected electric systems having an agreement to coordinate operations and planning for improved reliability and efficiencies.

Price: The amount of money or consideration-in-kind for which a service is bought, sold, or offered for sale.

Process Heating: Energy Efficiency program promotion of increased electric energy efficiency applications in industrial process heating.

Profit: The income remaining after all business expenses are paid.

Public Street and Highway Lighting: Public street and highway lighting includes electricity supplied and services rendered for the purposes of lighting streets, highways, parks, and other public places; or for traffic or other signal system service, for municipalities, or other divisions or agencies of State or Federal governments. Part 292.

Rate Base: The value of property upon which a utility is permitted to earn a specified rate of return as established by a regulatory authority. The rate base generally represents the value of property used by the utility in providing service and may be calculated by any one or a combination of the following accounting methods: fair value, prudent investment, reproduction cost, or original cost. Depending on which method is used, the rate base includes cash, working capital, materials and supplies, and deductions for accumulated provisions for depreciation, contributions in aid of construction, customer advances for construction, accumulated deferred income taxes, and accumulated deferred investment tax credits.

Ratemaking Authority: A utility commission's legal authority to fix, modify, approve, or disapprove rates, as determined by the powers given the commission by a State or Federal legislature.

Regulation: The governmental function of controlling or directing economic entities through the process of rulemaking and adjudication.
Reserve Margin (Operating): The amount of unused available capability of an electric power system at peak load for a utility system as a percentage of total capability.

Residential: The residential sector is defined as private household establishments which consume energy primarily for space heating, water heating, air conditioning, lighting, refrigeration, cooking and clothes drying. The classification of an individual consumer's account, where the use is both residential and commercial, is based on principal use. For the residential class, do not duplicate consumer accounts due to multiple metering for special services (water, heating, etc.). Apartment houses are also included.

Retail: Sales covering electrical energy supplied for residential, commercial, and industrial end-use purposes. Other small classes, such as agriculture and street lighting, also are included in this category.

Revenue: The total amount of money received by a firm from sales of its products and/or services, gains from the sales or exchange of assets, interest and dividends earned on investments, and other increases in the owner's equity except those arising from capital adjustments.

Sales: The amount of kilowatthours sold in a given period of time; usually grouped by classes of service, such as residential, commercial, industrial, and other. Other sales include public street and highway lighting, other sales to public authorities and railways, and interdepartmental sales.

Sales for Resale: Energy supplied to other electric utilities, cooperatives, municipalities, and Federal and State electric agencies for resale to ultimate consumers.

Standard Industrial Classification (SIC): A set of codes developed by the Office of Management and Budget, which categorizes business into groups with similar economic activities.

Standby Facility: A facility that supports a utility system and is generally running under no-load. It is available to replace or supplement a facility normally in service.

Standby Service: Support service that is available, as needed, to supplement a consumer, a utility system, or to another utility if a schedule or an agreement authorizes the transaction. The service is not regularly used.

System (Electric): Physically connected generation, transmission, and distribution facilities operated as an integrated unit under one central management, or operating supervision.

Total DSM Cost: Refers to the sum of total utility cost and nonutility cost. 
Total DSM Programs: Refers to the total net effects of all the utility's DSM programs. For the purpose of this survey, it is the sum of the effects for Energy Efficiency, Direct Load Control, Interruptible Load, Other Load Management, Other DSM Programs, and Load Building. Net growth in energy or load effects should be reported as a negative number, shown with a minus sign.

Total Nonutility Cost: Refers to total cash expenditures incurred by consumers and trade allies that are associated with participation in a DSM program, but that are not reimbursed by the utility. The nonutility expenditures should include only those additional costs necessary to purchase or install an efficient measure relative to a less efficient one. Costs are to be reported in nominal dollars in the year in which they are ?incurred, regardless of when the actual effects occur. To the extent possible, provide the best estimate of nonutility costs if actual costs are unavailable.

Total Utility Cost: Refers to the sum of the total Direct and Indirect Utility Costs for the year. Utility costs should reflect the total cash expenditures for the year, reported in nominal dollars, that flowed out to support DSM programs. They should be reported in the year they are incurred, regardless of when the actual effects occur.

Transmission: The movement or transfer of electric energy over an interconnected group of lines and associated equipment between points of supply and points at which it is transformed for delivery to consumers, or is delivered to other electric systems. Transmission is considered to end when the energy is transformed for distribution to the consumer.

Transmission System (Electric): An interconnected group of electric transmission lines and associated equipment for moving or transferring electric energy in bulk between points of supply and points at which it is transformed for delivery over the distribution system lines to consumers, or is delivered to other electric systems.

Uniform System of Accounts: Prescribed financial rules and regulations established by the Federal Energy Regulatory Commission for utilities subject to its jurisdiction under the authority granted by the Federal Power Act.

Utility-Earned Incentives: Costs in the form of incentives paid to the utility for achievement in consumer participation in DSM programs. These financial incentives are intended to influence the utility's consideration of DSM as a resource option by addressing cost recovery, lost revenue, and profitability.

Voltage Reduction: Any intentional reduction of system voltage by 3 percent or greater for reasons of maintaining the continuity of service of the bulk electric power supply system.

Water Heating: Energy Efficiency program promotion to increase efficiency in water heating, including low-flow shower heads and water heater insulation wraps. Could be applicable to residential, commercial, or industrial consumer sectors.

Watt: The electrical unit of power. The rate of energy transfer equivalent to 1 ampere flowing under a pressure of 1 volt at unity power factor.

Watthour (Wh): An electrical energy unit of measure equal to 1 watt of power supplied to, or taken from, an electric circuit steadily for 1 hour.

Wheeling Service: The movement of electricity from one system to another over transmission facilities of intervening systems. Wheeling service contracts can be established between two or more systems.

Wholesale Sales: Energy supplied to other electric utilities, cooperatives, municipals, and Federal and State electric agencies for resale to ultimate consumers. 



\title{
Appendix C
}

\author{
Abbreviations \\ and Acronyms
}





\section{Appendix C}

\section{Abbreviations and Acronyms}

CNEAF - Office of Coal, Nuclear, Electric and Alternate Fuels

DOE - Department of Energy

DSM - Demand-Side Management

EIA - Energy Information Administration

EPACT - Energy Policy Act of 1992

GWh - Gigawatthour

HVAC - Heating, Ventilation, and Air Conditioning

IRP - Integrated Resource Planning

KW - Kilowatt

kWh - Kilowatthour

MW - Megawatt
MWh - Megawatthour

NERC - North American Electric Reliability Council

The NERC regions are:

ASCC - Alaskan System Coordination Council

ECAR - East Central Area Reliability Coordination Agreement

ERCOT - Electric Reliability Council of Texas MAIN - Mid-America Interconnected Network

MAAC - Mid-Atlantic Area Council

MAPP - Mid-Continent Area Power Pool

NPCC - Northeast Power Coordinating Council SERC - Southeastern Electric Reliability Council SPP - Southwest Power Pool

WSCC - Western Systems Coordinating Council

NTIS - National Technical Information Service

TOU - Time-of-Use 
Energy Information Administration Consumption Surveys:

The Energy Information Administration (EIA) also conducts consumption surveys that provide detailed information on how different consumers use energy. In recent surveys, DSM data has been collected as part of the data collection for three EIA consumption surveys: the Residential Energy Consumption Survey, the Commercial Buildings Energy Consumption Survey, and the Manufacturing Energy Consumption Survey. The following provides a brief description of each of these surveys.

Residential Energy Consumption Survey (RECS): Since 1978, EIA has collected data from U.S. households about how they use energy and billing data from their energy suppliers about how much energy they use. In the ninth RECS undertaken in 1993, over 7,000 households were surveyed and the results are extrapolated to 97 million households. The triennial survey collects data on housing characteristics, energy consumption and expenditures, stock of energy-using appliances, and energy-related behavior.

Questions about household participation in DSM programs were asked in the 1990 and 1993 RECS. Data can be found in Housing Characteristics 1990 (DOE/EIA-0314(90)), Household Energy Consumption and Expenditures 1990 (DOE/EIA-0321(90)), and Housing Characteristics 1993 (tables available in November 1994 and report available in spring 1995). The data show participation by type of DSM program in both surveys. Additionally, the 1993 survey shows household perceptions of the availability of DSM programs.

For further information concerning the RECS DSM data or the RECS in general, please contact Wendel Thompson, RECS Manager, at (202) 586-1119, FAX at (202) 586-0018, or Internet E-mail wthompson @ eia.doe.gov.

Manufacturing Energy Consumption Survey (MECS): The MECS was first conducted for 1985 and presents data representing all but the smallest manufacturing establishments. It is a triennial survey that collects data on energy consumption and related issues in manufacturing establishments. The 1991 MECS presents separate estimates for all 20 major industrial groups from the manufacturing sector as defined by the Standard Industrial Classification (SIC) Codes. Within these major groups, separate estimates are presented for $\mathbf{4 2}$ industries and industry groups.

New to the 1991 version of the MECS are data on energy efficiency activities and DSM in particular. The data tables are available now in electronic form on EPUBS and in a forthcoming publication. The tables present participation by SIC Code, type of program, and whether electric utilities are involved. Due to the sample design, data must be presented in terms of energy consumption rather than counts of establishments. In future years, both types of measures are expected to be available.

For further information concerning DSM data or any aspect of the MECS, please contact Mark Shipper, MECS Survey Manager, at (202) 586-1136, FAX at (202) 586-0018, or Internet E-mail mshipper@ eia.doe.gov.

Commercial Buildings Energy Consumption Survey (CBECS):Since 1979, EIA has collected data on the physical and operating characteristics that affect energy use in U.S. commercial buildings. Billing data containing energy consumption and expenditures are collected from the energy suppliers to these buildings. In the fifth CBECS undertaken in 1992, both the building respondents and the energy suppliers were asked extensive questions about the types of DSM programs that the buildings participated in, the sponsors of those programs, and the types of assistance that was provided through the DSM programs. DSM participation data as reported by the building owners, managers, and tenants can be found in Commercial Buildings Characteristics 1992 (DOE/EIA-0246(92)).

For further information concerning the CBECS DSM data or the CBECS in general, please contact Martha Johnson, CBECS Manager, at (202) 586-1135, FAX at (202) 586-0018, or Internet E-mail mjohnson @ eia.doe.gov. 


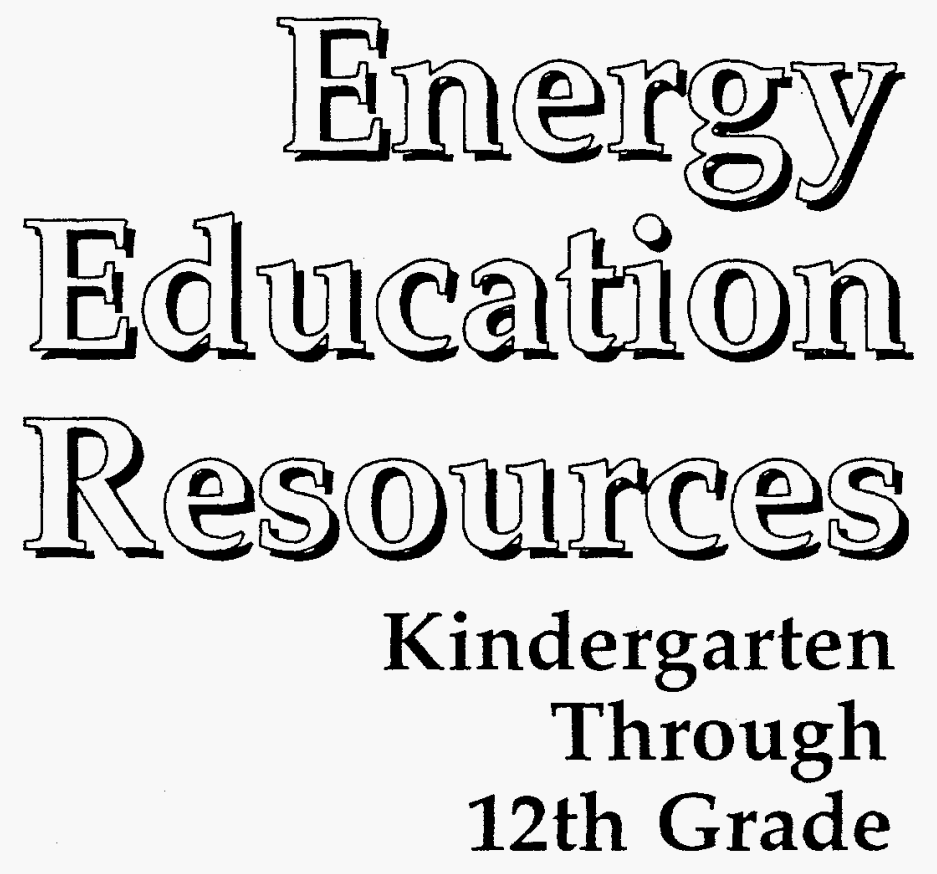

This brochure contains a listing of free or low-cost energy-related educational materials for educators and primary and secondary students.

\section{Call the National Energy Information Center on (202)586-8800 to order your free copy.}

